\title{
OPTIMIZING TREATMENT FOR OBSTRUCTIVE COLON CANCER
}

FEMKE JULIE AMELUNG 



\section{OPTIMIZING TREATMENT FOR OBSTRUCTIVE COLON CANCER}

FEMKE JULIE AMELUNG 



\title{
OPTIMIZING TREATMENT FOR OBSTRUCTIVE COLON CANCER
}

\author{
PROEFSCHRIFT \\ ter verkrijging van de graad van \\ doctor aan de Universiteit Twente, \\ op gezag van de rector magnificus, \\ prof. dr. T.T.M. Palstra, \\ volgens besluit \\ van het College voor Promoties \\ in het openbaar te verdedigen \\ op vrijdag 23 november 2018 om 14.45 uur
}

door

FEMKE JULIE AMELUNG

geboren op 18 juni 1990

te Utrecht 
Promotoren: $\quad$ Prof. dr. P.D. Siersema

Prof. dr. I.A.M.J. Broeders

Copromotor: Dr. E.C.J. Consten

Commissieleden: Prof. dr. T.T.M. Palstra

Prof. dr. R.H. Geelkerken

Prof. dr. G.L. Beets

Prof. dr. J.H.W. de Wilt

Dr. E. Groot Jebbink

Dr. P.J. Tanis

Dr. J.E. van Hooft

Copyright (C) F.J. Amelung 2018

No part of this thesis may be reproduced in any form, by print, photocopy, electronic data transfer or any other means, without prior permission of the author.

ISBN

978-90-365-4652-2

Cover

Franka Würdemann

Layout and design

wenz iD \| Wendy Schoneveld

Printed by

GVO drukkers

Printing of this thesis was financially supported by: Meander Medical Center | Chipsoft | Universiteit Twente | Nederlandse Vereniging van Endoscopische Chirurgie | KARL STORZ Endoscopie Nederland B.V. | ABN AMRO bank 
Voor mijn ouders 


\section{Contents}

Chapter 1 General introduction and outline of this thesis

\section{PART I RIGHT-SIDED COLONIC OBSTRUCTIONS}

Chapter 2 Emergency resection versus bridge to surgery with stenting in patients with acute right-sided colonic obstruction: a systematic review focusing on mortality and morbidity rates International Journal of Colorectal Disease 2015

Chapter 3 A population based analysis of three treatment modalities for malignant 39 obstruction of the proximal colon: acute resection versus stent or stoma as a bridge to surgery

Annals of Surgical Oncology 2016

Chapter 4 Self-expandable metal stent placement versus emergency resection for 53 malignant proximal colon obstructions

Surgical Endoscopy 2017

\section{PART II LEFT-SIDED COLONIC OBSTRUCTIONS}

Chapter 5 Acute resection versus bridge to surgery with diverting colostomy for patients with acute malignant left sided colonic obstruction: systematic review and meta-analysis

Surgical Oncology 2015

Chapter 6 Efficacy of loop colostomy construction for acute left-sided colonic 91 obstructions: a cohort analysis International Journal of Colorectal Disease 2017

Chapter 7 Deviating colostomy versus stent placement as bridge to surgery for malignant left-sided colonic obstruction

Surgical Endoscopy 2016

Chapter 8 lleostomy versus colostomy: which is preferable? 


\section{PART III LONG-TERM OUTCOMES}

Chapter 9 Long-term outcomes following stent placement as bridge to surgery compared to emergency surgery in patients with acute left-sided colonic obstruction; an updated meta-analysis Critical Reviews in Hematology / Oncology 2018

Chapter 10 A propensity score matched analysis of oncological outcomes between stent as bridge to surgery and emergency resection for left-sided obstructive colon cancer

Submitted

Chapter 11 Incidence and risk factors of ostomy site incisional hernia following stoma reversal

British Journal of Surgery Open 2018

\section{PART IV APPENDICES}

Chapter 12 Summary, discussion and future perspectives 



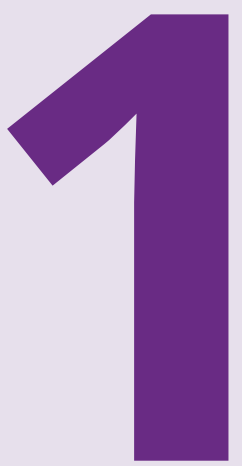

General introduction and outline 
Colon cancer is the third most common cancer worldwide and it's incidence is annually increasing. ${ }^{1}$ In the Netherlands alone, over 15.000 new cases are diagnosed each year. ${ }^{2}$ Most of these patients present with non-specific symptoms such as fatigue, weight loss or a changed stool pattern. Further diagnostic tests can then lead to the diagnosis of colorectal cancer. ${ }^{3}$ In nine to thirteen percent of patients with colorectal cancer however, acute colonic obstruction is the first symptom. ${ }^{4}$ These patients require immediate treatment and acute malignant obstructions account for $85 \%$ of colonic emergencies. ${ }^{5}$ These numbers clearly show obstructive colon cancer to be a significant problem.

The majority of patients presenting with an acute colonic obstruction are elderly and in a poor clinical condition due to several days to weeks of reduced intake and significant weight loss. ${ }^{6,7}$ Furthermore, presentation with acute colonic obstruction is associated with more advanced disease and a higher incidence of metastatic disease..$^{8-10}$ These factors all contribute to an increased peri- and postoperative surgical risk. ${ }^{11}$ On the other hand, when total obstruction is present immediate intervention is required to prevent life-threatening complications due to extensive bowel dilation.

Currently, emergency resection is the most applied treatment approach, even though this procedure is associated with morbidity and mortality rates up to $60 \%$ and $22 \%$, respectively. ${ }^{11}$ The results of a recent national audit on acute colonic obstructions report on slightly better outcomes with $42.8 \%$ morbidity and $6.9 \%$ mortality. In high-risk patients (ASA >2 and age $>70$ years), however, mortality still remains as high as $32.2 \% .{ }^{12}$ This can be contributed to the deteriorated clinical condition of the patients, their poor nutritional state and the invasiveness of the surgery since a laparoscopic approach is often impossible due to severely distended bowel. These distended bowels also hamper primary anastomosis construction and many patients end up with a permanent colostomy, which is associated with frequent complications and a lower quality of life. ${ }^{13,14}$

These outcomes have prompted the search for a valid and safe alternative. This alternative has possibly been found in a bridge to surgery (BTS) approach, with the aim to achieve initial colonic decompression using a minimally invasive procedure. Hereby, the risks of colonic obstruction are addressed immediately and fecal passage is restored with the objective to reduce bowel wall distention. This creates time to optimize the patients' condition prior to elective resection and to perform adequate tumor staging. By doing this, extensive surgery in patients with disseminated disease or unacceptable surgical risk can be avoided, since a BTS approach can then serve as a definite palliative measure.$^{15}$ Colonic decompression as bridge to surgery can be achieved by construction of a deviating stoma (DS) or by placement of a self-expandable metallic stent (SEMS).

\section{Self-expandable metal stent (SEMS) as bridge to surgery (figure 1)}

In 1991, Dohmoto first proposed placement of a self-expandable metallic stent (SEMS) to relief colonic obstruction. ${ }^{16}$ Primarily, SEMS were mainly used in palliative patients in order to spare them from major abdominal surgery and possible colostomy construction. ${ }^{17}$ In recent years, 
FIGURE 1 | A Passing the stent and guidewire through the lesion with contrast injection. B Partial stent deployment. C Pull back stent and scope until fair part of stent reach upper border. D Full deployment of SEMS. (adapted from van Halsema et al. ${ }^{53}$ )
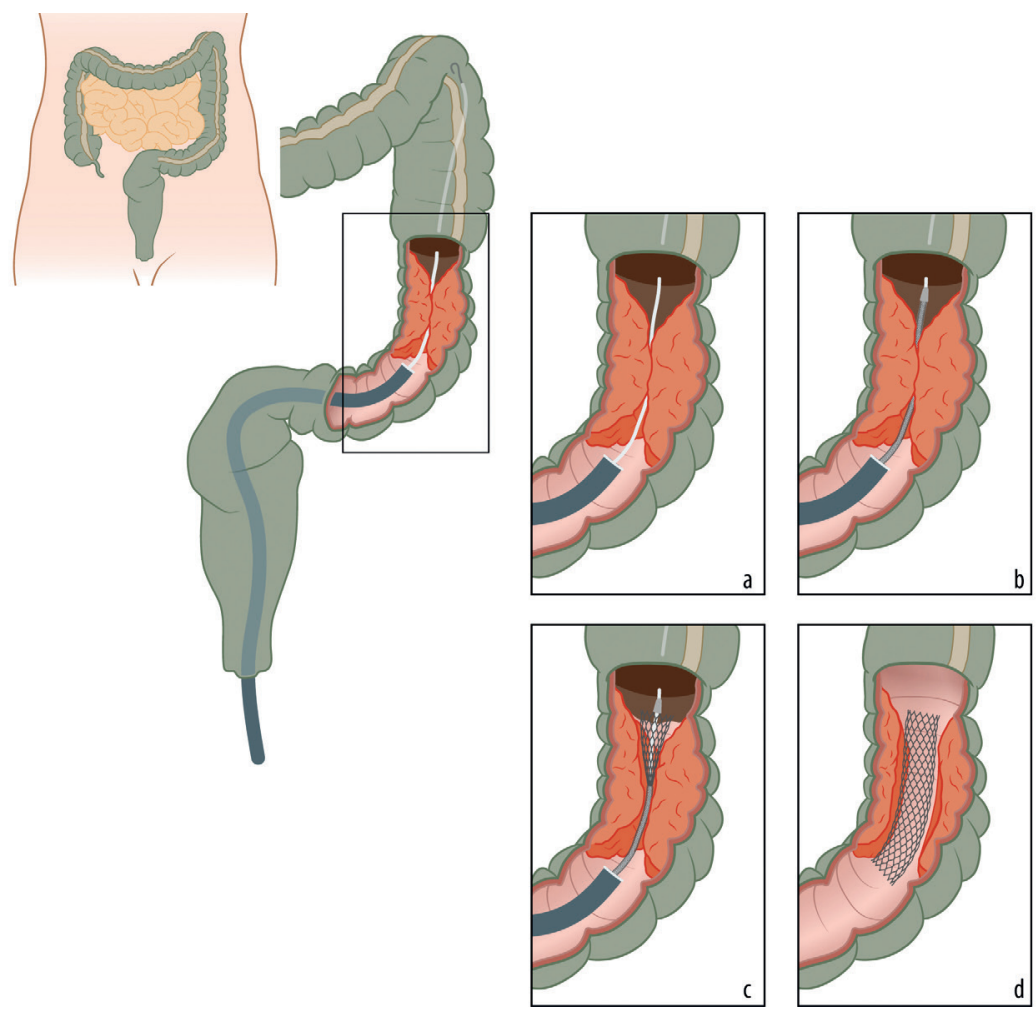

however, SEMS placement has also been used as a bridge to elective surgery in curative patients. ${ }^{18,19}$ Almost 30 years after its first introduction, the short-term benefits of SEMS as bridge to surgery have been well established. Even though several recent meta-analyses could not confirm an impact on postoperative mortality rates, they did reveal a higher likelihood of undergoing laparoscopic surgery, lower morbidity rates, fewer temporary stoma constructions and higher primary anastomoses rates. ${ }^{20,21}$ In addition, permanent stoma rates are significantly lower.

These favorable short-term outcomes make SEMS seem as an attractive alternative to emergency resection. However, SEMS has been under debate recently, especially in curative patients, since it has been suggested to negatively influence oncologic outcomes. ${ }^{22}$

In addition, the high perforation rates of around $8-10 \%$ are an important cause of concern. ${ }^{20,23}$ Long-term results of the Stent-in II trial have also shown higher local recurrence rates in patients that suffered a guidewire or SEMS-related perforation. ${ }^{24,25}$ The use of SEMS in curative patients is currently only recommended in high-risk patients with an ASA score of 3 or higher and/or aged above 70 years. ${ }^{26}$ 
FIGURE 2 | Construction of a deviating colostomy. A Small incision in de right upper quadrant. B Opening of the colon and attach colon mucosa to the skin. C placement of temporary stoma bridge. (adapted from van Halsema et al..$^{53}$ )
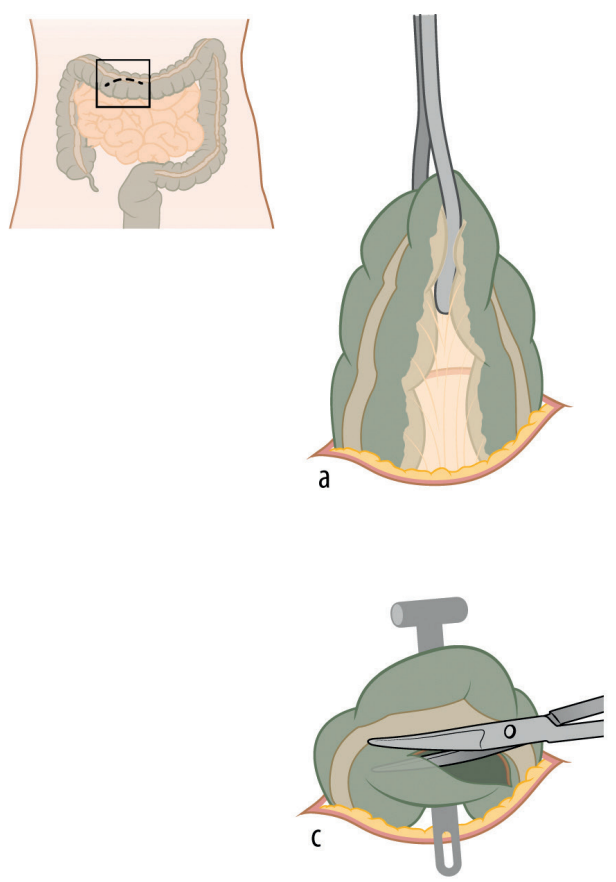
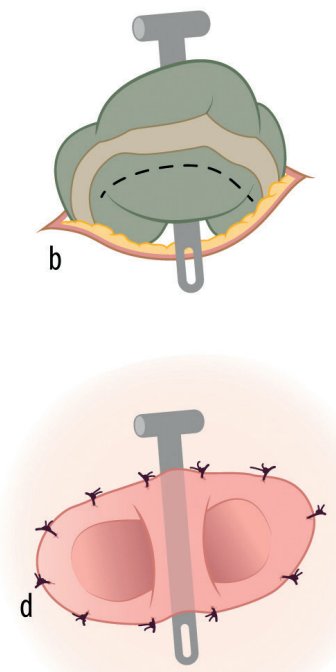

\section{Deviating colostomy as bridge to surgery}

Construction of a deviating colostomy ensures efficient fecal diversion and decompression of the colon. Using the trephine method, a loop colostomy on the transverse colon is constructed in a minimally invasive manner without the need for a laparotomy. According to this method, a transverse colon loop is externalized through a small incision in the upper right abdominal quadrant, after which the stoma can be constructed (Figure 2). ${ }^{27}$ Another possibility is construction of a blowhole. As well as with deviating colostomy construction, a small incision in the upper right quadrant of the abdomen is made. However, the transverse colon is not elevated with this technique, but immediately secured to the posterior fascia (creating a seal which prevents spillage of enteric content). Hereafter, the transverse colon is opened, whereafter a blowhole is constructed by attaching the colonic mucosa to the dermis (Figure 3). ${ }^{28}$ Blowhole construction is considered easier and can be conducted under local anesthesia, however, it is also a relatively new technique and data is scarce. With both techniques, the aim of stoma construction is to prevent complications due to bowel distention, while providing the aforementioned benefits of a bridge to surgery approach. Indeed, significantly fewer permanent stomas are reported when patients are primarily treated with a (deviating) stoma as bridge to surgery compared to emergency surgery..$^{29}$ 
FIGURE 3 | Construction of a blowhole. A Securing the transverse colon to the posterior fascia. B Securing the mucosa to the dermis, finalizing the blowhole. (adapted from Kasten et al. ${ }^{29}$ )
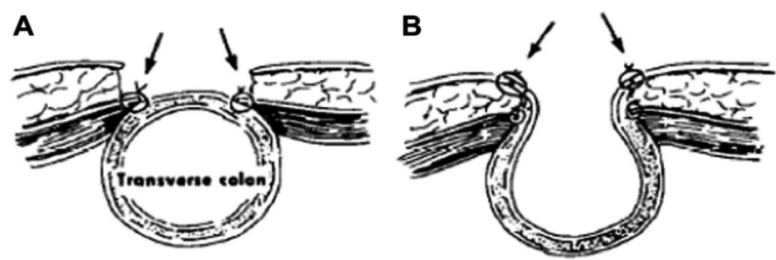

In addition, since the tumor is not manipulated directly such as is the case with SEMS placement, no effect on long-term oncologic outcomes is expected..$^{29}$ Deviating stoma construction as BTS has been abandoned in the past because of presumed prolonged hospital stay, increased number of surgical interventions and a possible negative effect on quality of life. However, studies are limited and outdated and it may be questioned if this approach is inferior to SEMS when performed according to the current standard of care.

Interestingly, even though literature on SEMS placement for obstructive colon cancer is extensive, very few studies have compared SEMS to a deviating colostomy. Therefore, it remains unclear which bridge to surgery approach should be preferred.

\section{PART I - RIGHT-SIDED COLONIC OBSTRUCTION}

Treatment approaches for obstructive colon tumors differ according to their location. When the tumor is located in the ascending colon, hepatic flexure or transverse colon it is classified as a 'right-sided' tumor. Tumors in the descending colon, sigmoid or splenic flexure are classified as 'left-sided'.

Most of the obstructing tumors are located in the distal colon, since the colon narrows and feces becomes more solid. As a consequence, most studies concerning treatment of obstructive colon cancer report on left-sided obstructions. However, still approximately $33-54 \%$ of obstructing tumors are located proximal to the splenic flexure. ${ }^{30}$ Most surgeons agree that primary resection is justified for the majority of patients with malignant proximal obstructions. ${ }^{31}$ This is mainly because postoperative outcomes of emergency surgery in the proximal colon were comparable with elective surgery in previous older reports. ${ }^{32}$ Recent studies have shown mortality rates following emergency resection in patients with a right-sided colonic obstruction to be significantly higher than previously thought. ${ }^{8,10,33}$ Initial colonic decompression using a minimally invasive procedure as bridge to surgery might therefore also benefit patients with a right-sided obstruction. However, when looking at all available literature on bridge to surgery approaches, less then $5 \%$ of the studies involve the proximal colon. ${ }^{34}$

In order to investigate the evidence currently available on bridge to surgery approaches for right-sided obstructions, we conducted a systematic review with the results being presented 
in Chapter 2. In Chapter 3, a national cohort study is presented, aiming to determine which treatment approaches are currently used in the Netherlands. In addition, the corresponding outcomes for the different treatment strategies are reported.

While SEMS placement has become more and more accepted for left-sided obstructions, data on its safety in more proximal obstructions is scarce. Two previous studies have shown promising results, but both studies were of limited methodological quality and definitive conclusions cannot be drawn based on these outcomes. ${ }^{35,36}$ In an attempt to provide more solid data we conducted a case-matched analysis of short- and long-term outcomes, comparing emergency surgery and SEMS placement. The results are presented in Chapter 4.

\section{PART II - LEFT-SIDED COLONIC OBSTRUCTION}

As mentioned previously, most obstructing tumors are located in the left colon. ${ }^{30}$ Literature on emergency resection versus SEMS placement as BTS in left-sided obstructions is therefore extensive. Nonetheless, studies reporting on deviating stoma construction are scarce and of limited quality. In Chapter $\mathbf{5}$ we aim to provide an overview of all available literature concerning deviating stoma construction as bridge to surgery through a systematic review of the literature. At Meander Medical Center, construction of a deviating stoma was traditionally the first treatment choice for left-sided colonic obstructions. In order to further investigate the safety of colostomy construction as bridge to surgery with the current standard of care, the results of a cohort analysis of all patients treated with a colostomy for obstructive colon cancer between 2003 and 2015 are presented in Chapter 6.

Even though bridge to surgery approaches have gained popularity and are increasingly applied, data investigating which approach is superior is lacking. Most existing studies solely compare SEMS or deviating stoma with emergency resection. A recent Dutch national cohort study is the first to compare all three treatment options. ${ }^{12}$ Interestingly, both bridge to surgery groups showed favorable short-term outcomes compared to emergency surgery. Unfortunately, p-values specifically comparing the bridge to surgery groups are lacking for most variables. Only 30-day or in hospital mortality rates following elective resection were directly compared between SEMS and deviating stoma construction. A trend towards lower mortality was seen in the stoma group ( 4.3 vs. $1.8 \%, p=0.051$ ).${ }^{12}$ In addition, it is hypothesized that no long-term oncologic risks are associated with the construction of a stoma. ${ }^{28}$ On the other hand, SEMS placement might prevent stoma construction, which could be beneficial for the quality of life of patients. Both bridge to surgery approaches therefore seem to have their own (dis) advantages, but no definite (evidence-based) consensus exists on which treatment is preferred. Therefore, a retrospective multi-institutional cohort analysis was conducted, aiming to compare short- and long-term outcomes of SEMS versus deviating stoma, of which the results are reported in Chapter 7.

Another important topic for patients with a colonic obstruction is stoma construction. During the entire treatment course, many patients will be confronted with (temporary) stoma 
construction. A stoma can be constructed either in order to protect a newly constructed anastomosis following (emergency) resection or in order to decompress the colon. ${ }^{37}$ Stoma construction is associated with a high complication rate and is known to negatively impact health-related quality of life. ${ }^{13} \mathrm{~A}$ Dutch prospective study of 100 patients showed an overall stoma-related complication rate of $82 \%{ }^{38}$ In order to make sure complications are kept to a bare minimum, it is important to construct the most optimal stoma type. When a stoma is constructed, the surgeon can choose between construction of a loop ileostomy or colostomy. The current Dutch guidelines do not recommend a specific type. ${ }^{39}$ The American Society of Colon and Rectal Surgeons hints towards a slight preference for ileostomy construction, since reversal is thought to be easier and stoma prolapse is less common. However, they also state that a loop ileostomy is more likely to result in a high output stoma, which could result in dehydration. Therefore, no clear preference is stated..$^{40}$ in Chapter 8 we aim to investigate which type of loop stoma should be preferred in patients receiving a temporary stoma. This was investigated though a systematic review of all available literature and complemented with a retrospective analysis of our own data.

\section{PART III - LONG-TERM OUTCOMES}

As mentioned previously, the short-term benefits of SEMS placement have been well established in recent years. ${ }^{20,21}$ Despite this, stent placement in curative patients has been used with reserve due to uncertainty about its impact on long-term oncological outcomes. ${ }^{22}$ This originates from concerns about tumor manipulation during stent insertion, guidewire perforations during stent placement, stent deployment force and possible micro-perforations at the proximal and distal ends of the stent, which may induce tumor cell dissemination locally, but also in the blood stream. In 2007, Marutchalam et al. found an increase of cytokeratin 20mRNA expression (a marker for circulating tumor cells) in peripheral venous blood following SEMS placement. ${ }^{41} \mathrm{~A}$ few years later, two studies showed a higher rate of perineural and lymph node invasion after SEMS placement. ${ }^{42,43}$ The clinical relevance of these surrogate outcome measures remained, however, uncertain. We therefore performed a systematic review and subsequent meta-analysis investigating long-term oncological safety of SEMS placement. Chapter 9 contains the outcomes of this study.

Unfortunately, most studies included in Chapter 9 were retrospective, included a low number of patients, long-term oncological outcomes were seldom the primary outcome measure and follow-up was short. The results were therefore found to be of limited strength. In order to provide more solid, real-world evidence ${ }^{44}$ regarding this topic, in Chapter 10 we assess longterm oncological outcomes following SEMS placement using a propensity score matched population-based analysis.

Even though oncological safety following SEMS placement is a hot topic, no such concerns have been raised regarding deviating stoma construction. Stoma construction, however, has been associated with increased surgical procedures in the past. For example, in order to reverse 
the stoma, the patients should undergo at least one re-operation. Overall, stoma reversal is regarded as a straightforward and safe procedure with low morbidity and mortality rates ${ }^{45}$ In the long run, however, the stoma incision site is at risk for incisional hernia development, which could require additional surgeries. ${ }^{46}$ Early studies have estimated incisional hernia incidences following stoma reversal at approximately 7\%. ${ }^{47-49}$ Recent studies specifically designed to investigate stoma site herniation have reported a considerably higher incidence of 30-35\% ${ }^{50-52}$ Incisional hernias following stoma closure may therefore be an underestimated clinical problem..$^{50}$ They can cause abdominal pain, discomfort and impaired quality of life. Medical consultation is often sought and approximately $50 \%$ of the patients require surgical correction to relief symptoms. ${ }^{50}$ In Chapter 11, we focused on investigating long-term outcomes following stoma reversal, with incisional hernia development as the primary outcome. In addition, we aimed to identify possible risk factors for incisional hernia development in order to optimize treatment strategies. 


\section{REFERENCES}

1. World Cancer Research Fund International (WCRF). Worldwide cancer registry. https://www.wcrf.org/

2. The Netherlands Comprehensive Cancer Organisation (IKNL). The Netherlands Cancer Registry. http://www. cijfersoverkanker.nl/

3. Astin M, Griffin T, Neal RD, Rose P, Hamilton W. The diagnostic value of symptoms for colorectal cancer in primary care: a systematic review. BJGP. 2011;61(586):e231-e243

4. Jullumstro E, Wibe A, Lydersen S, Edna TH. Colon cancer incidence, presentation, treatment and outcomes over 25 years. Colorectal Dis 2011;13(5):512-518

5. Yeo HL, Lee SW. Colorectal emergencies: review and controversies in the management of large bowel obstruction. J Gastrointest Surg 2013;17:2007-12

6. Scott NA, Jeacock J, Kingston RD. Risk factors in patients presenting as an emergency with colorectal cancer. BJS 1995;82(3):321-3

7. Hennessey DB, Burke JP, Ni-Dhonochu T, Shields C, Winter DC, Mealy K. Preoperative hypoalbuminemia is an independent risk factor for the development of surgical site infection following gastrointestinal surgery: a multi-institutional study. Ann Surg 2010;252(2):325-9

8. Sjo OH, Larsen S, Lunde OC, Nesbakken A. Short term outcome after emergency and elective surgery for colon cancer. Colorectal Dis. 2009;11:733-739

9. Papadimitriou G, Manganas D, Phedias Georgiades C, Vougas V, Vardas K, Drakopoulos S. Emergency surgery for obstructing colorectal malignancy: prognostic and risk factors. J BUON. 2015;20(2):406-12

10. Smothers L, Hynan L, Fleming J, Turnage R, Simmang C, Anthony T. Emergency surgery for colon carcinoma. DCR 2003;46(1):24-30

11. Iversen LH, Bülow S, Christensen IJ, Laurberg S, Harling $\mathrm{H}$. Postoperative medical complications are the main cause of early death after emergency surgery for colonic cancer. BJS. 2008;95:1012-9

12. Tanis PJ, Paulino Pereira NR, van Hooft JE, Consten EC, Bemelman WA. Resection of Obstructive Left-Sided Colon Cancer at a National Level: A Prospective Analysis of Short-Term Outcomes in 1,816 Patients. Dig Surg. 2015;32(5):317-324

13. Young CJ, De-Loyde KJ, Young JM, Solomon MJ, Chew EH, Byrne CM, Salkeld G, Faragher IG. Improving Quality of Life for People with Incurable Large-Bowel Obstruction: Randomized Control Trial of Colonic Stent Insertion. Dis Colon Rectum. 2015;58(9):838-49
14. Anaraki F, Vafaie M, Behboo R, Maghsoodi N, Esmaeilpour S, Safaee A. Quality of life outcomes in patients living with stoma. Indian J Palliat Care. 2012;18(3):176-80

15. Bhardwaj R, Parker MC. Palliative therapy of colorectal carcinoma: stent or surgery? Colorectal Dis. 2003;5(5):518-21

16. Dohmoto M. New method: endoscopic implantation of rectal stent in palliative treatment of malignant stenosis. Endosc Dig. 1991;3:1507-12

17. Dohmoto $M$, Hünerbein $M$, Schlag PM. Application of rectal stents for palliation of obstructing rectosigmoid cancer. Surg Endosc. 1997;11(7):758-61

18. Vitale MA, Villotti G, d'Alba L, Frontespezi S, lacopini F, lacopini G. Preoperative colonoscopy after selfexpandable metallic stent placement in patients with acute neoplastic colon obstruction. Gastrointest Endosc. 2006;63:814-9

19. Saida $Y$, Sumiyama $Y$, Nagao J, Takase M. Stent endoprothesis for obstructing colorectal cancers. Dis Colon Rectum 1996;39:552-5

20. Arezzo A, Passera R, Lo Secco G, Verra M, Bonino MA, Targarona $\mathrm{E}$, Morino $\mathrm{M}$. Stent as bridge to surgery for left-sided malignant colonic obstruction reduces adverse events and stoma rate compared with emergency surgery: results of a systematic review and meta-analysis of randomized controlled trials. Gastrointest Endosc. 2017; 86:416-426

21. Allievi N, Ceresoli M, Fugazzola P, Montori G, Coccolini $\mathrm{F}$, Ansaloni L. Endoscopic Stenting as Bridge to Surgery versus Emergency Resection for Left-Sided Malignant Colorectal Obstruction: An Updated Meta-Analysis. Int J Surg Oncol. 2017;2863272

22. Sabbagh C, Browet F, Diouf $\mathrm{M}$ et al. Is stenting as "a bridge to surgery" an oncologically safe strategy for the management of acute, left-sided, malignant, colonic obstruction? A comparative study with a propensity score analysis. Ann Surg 2013;258:107-115

23. van Hooft JE, Bemelman WA, Oldenburg B, et al. Colonic stenting versus emergency surgery for acute left-sided malignant colonic obstruction: a multicentre randomised trial. Lancet Oncol 2011;12:344-52

24. Avlund TH, Erichsen R, Ravn S, Ciplys Z, Andersen JC, Laurberg S, Iversen LH. The prognostic impact of bowe perforation following self-expanding metal stent as a bridge to surgery in colorectal cancer obstruction. Surg Endosc 2018;32;328-336

25. Sloothaak DA, van den Berg MW, Dijkgraaf MG, Fockens 
P, Tanis PJ, van Hooft JE, Bemelman WA. Oncological outcome of malignant colonic obstruction in the Dutch Stent-In 2 trial. Br J Surg 2014;01(13):1751-7

26. van Hooft JE, van Halsema EE, Vanbiervliet G, Beets-Tan RG, DeWitt JM, Donnellan F, Dumonceau JM, GlynneJones RG, Hassan C, Jime'nez-Perez J, Meisner S, Muthusamy VR, Parker MC, Regimbeau JM, Sabbagh C, Sagar J, Tanis PJ, Vandervoort J, Webster GJ, Manes G, Barthet MA, Repici A. Selfexpandable metal stents for obstructing colonic and extracolonic cancer: European Society of Gastrointestinal Endoscopy (ESGE) Clinical Guideline. Endoscopy. 2014;46(11):990-1053

27. Nylund G, Oresland T, Hultén L. The trephine stoma: formation of a stoma without laparotomy. Eur J Surg. 1997;163:627-9

28. Jiang JK, Lan YT, Lin TC, Chen WS, Yang SH, Wang HS, Chang SC, Lin JK. Primary vs. delayed resection for obstructive left-sided colorectal cancer: impact of surgery on patient outcome. Dis Colon Rectum. 2008;51(3):306-11

29. Kasten KR, Midura EF, Davis BR, Rafferty JF, Paquette IM. Blowhole colostomy for the urgent management of distal large bowel obstruction. J Surg Res. 2014 May $1 ; 188(1): 53-7$

30. Lee YM, Law WL, Chu KW, Poon RT. Emergency surgery for obstructing colorectal cancers: a comparison between right-sided and left-sided lesions. J Am Coll Surg. 2001;192(6):719-25

31. Phillips RKS, Hittinger R, Fry JS, Fielding LP. Malignant large bowel obstruction. Br J Surg. 1985;72:296-302

32. Smithers BM, Theile DE, Cohen JR, Evans EB, Davis NC.Emergency right hemicolectomy in colon carcinoma: a prospective study. Aust N Z J Surg. 1986;56:749-52

33. Kobayashi $\mathrm{H}$, Miyata $\mathrm{H}$, Gotoh $\mathrm{M}$, et al. Risk model for right hemicolectomy based on 19,070 Japanese patients in the National Clinical Database. J Gastroenterol. 2014;49(6):1047-55

34. Bonin EA, Baron TH. Update on the Indications and Use of Colonic Stents. Curr Gastroenterol Rep 2010 12(5):374-382)

35. Hsu TC. Comparison of one-stage resection and anastomosis of acute complete obstruction of left and right colon. Am J Surg. 2005;189:384-7

36. Campbell KL, Hussey JK, Eremin O. Expandable metal stent application in obstructing carcinoma of the proximal colon: report of a case. Dis Colon Rectum. 1997;40:1391-3

37. Scott-Conner, CEH. Chassin's operative strategy in colon and rectal surgery. New York: Springer; 2006:18
38. Formijne Jonkers HA, Draaisma WA, Roskott AM, van Overbeeke AJ, Broeders IA, Consten EC. Early complications after stoma formation: a prospective cohort study in 100 patients with 1-year follow-up. Int J Colorectal Dis. 2012;27:1095-9.

39. Landelijke werkgroep Gastro Intestinale Tumoren. Richtlijn colorectaal carcinoom, versie 3.0. Utrecht IKNL; 2014

40. Monson JRT, Weiser MR, Buie WD, Chang GJ, Rafferty JF. Practice parameters for the management of rectal cancer (revised). Dis Colon Rectum. 2013;56:535-50

41. Maruthachalam K, Lash GE, Shenton BK, Horgan AF. Tumour cell dissemination following endoscopic stent insertion. Br J Surg 2007;94:1151-1154, doi: NA

42. Kim HJ, Choi GS, Park JS et al. Higher rate of perineura invasion in stent-laparoscopic approach in comparison to emergent open resection for obstructing left-sided colon cancer. Int J Colorect Dis 2013;28:207-14

43. Poon J. Pang R. Law W. The impact of colonic stenting on tumor cell dissemination in colorectal cancer patients. Dis.Colon Rectum 2011;54:160

44. Sherman RE, Anderson SA, Dal Pan GJ, Gray GW, Gross T, Hunter NL, LaVange L, Marinac-Dabic D, Marks PW, Robb MA, Shuren J, Temple R, Woodcock J, Yue LQ Califf RM. Real-World Evidence - What Is It and What Can It Tell Us? N Engl J Med. 2016;375(23):2293-2297

45. Chow A, Tilney HS, Paraskeva P, Jeyarajah S, Zacharakis $\mathrm{E}$, Purkayastha $\mathrm{S}$. The morbidity surrounding reversal of defunctioning ileostomies: a systematic review of 48 studies including 6107 cases. Int J Colorectal Dis 2009;24: 711-723

46. Cingi A, Cakir T, Sever A, Aktan AO. Enterostomy site hernias: a clinical and computerized tomographic evaluation. Dis Colon Rectum 2006;49:1559-1563

47. Tekkis PP. Comparison of outcomes following ileostomy versus colostomy for defunctioning colorectal anastomoses. World J Surg 2007;31:1142-1151

48. Bakx R, Busch OR, BemelmanWA, Veldink GJ, Slors JF, van Lanschot JJ. Morbidity of temporary loop ileostomies. Dig Surg 2004;21:277-281

49. Guenaga KF, Lustosa SA, Saad SS, Saconato H, Matos D. lleostomy or colostomy for temporary decompression of colorectal anastomosis. Cochrane Database Syst Rev 2007;(1)CD004647

50. Bhangu A, Nepogodiev D, Futaba K. Systematic review and meta-analysis of the incidence of incisional hernia at the site of stoma closure. World J Surg 2012;36:973983 
51. Bhangu A, Fletcher L, Kingdon S, Smith E, Nepogodiev D, Janjua U. A clinical and radiological assessment of incisional hernias following closure of temporary stomas. Surgeon 2012;10:321-325

52. Schreinemacher MH, Vijgen GH, Dagnelie PC, Bloemen $J G$, Huizinga BF, Bouvy ND. Incisional hernias in temporary stoma wounds: a cohort study. Arch Surg 2011;146: 94-99

53. van Halsema E, Tanis PJ, ter Borg F, Consten ECJ, Bemelman WA, van Hooft JE. Stent, stoma of acute resectie bij colonobstructie? NtvG 2015;159:A8795 


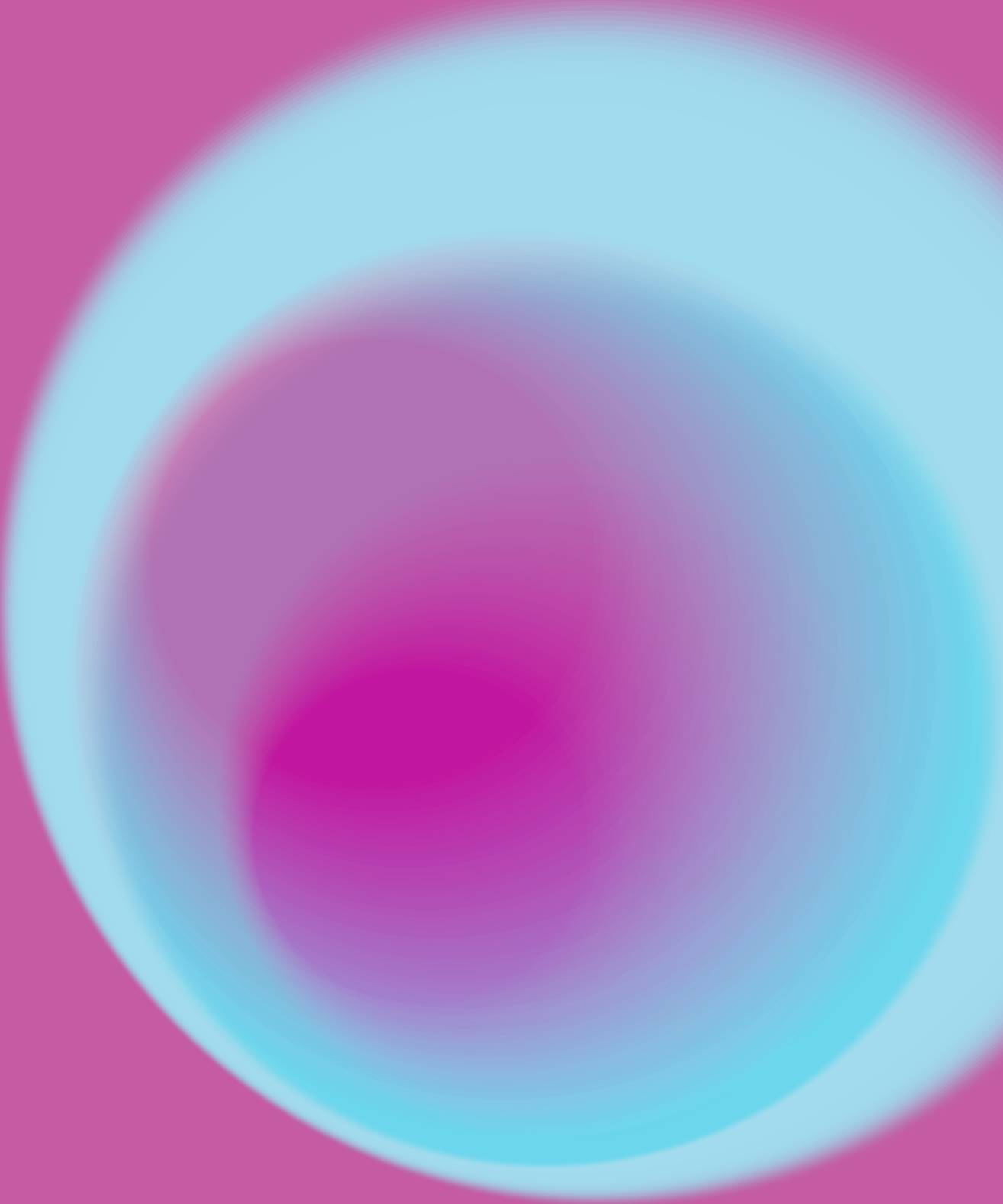





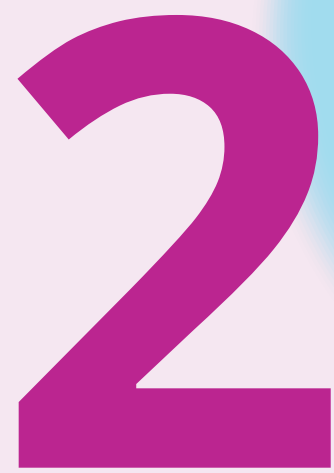

Emergency resection versus bridge to surgery with stenting in patients with acute right-sided colonic obstruction: a systematic review focusing on mortality and morbidity rates

International Journal of Colorectal Disease 2015

\author{
F.J. Amelung \\ H. de Beaufort \\ P.D. Siersema \\ P.M. Verheijen \\ E.C.J. Consten
}




\section{ABSTRACT}

\section{Purpose}

No consensus exists on the optimal treatment of acute malignant right-sided colonic obstruction (RSCO). This systematic review aims to compare procedure-related mortality and morbidity rates between primary resection and stent placement as a bridge to surgery followed by elective resection for patients with acute RSCO.

\section{Methods}

Pubmed, Embase and Cochrane library were searched for all relevant literature. Primary endpoints were procedure-related mortality and morbidity. Methodological quality of the included studies was assessed using the MINORS criteria.

\section{Results}

Fourteen cohort studies were eligible for analysis. A total of 2873 patients were included in the acute resection group and 155 patients in the stent group. Mean mortality rate for patients who underwent acute resection with primary anastomosis was $10.8 \%$ (8.1\%-18.5\%). Overall mortality for patients initially treated with a colonic stent followed with elective resection was $0 \%$. Major morbidity was $23.9 \%(9.3 \%-35.6 \%)$ and $0.8 \%(0 \%-4.8 \%)$, respectively. Both mortality and major morbidity were significantly different. In addition, stent placement shows lower rates of anastomotic leakages ( $0 \%$ vs $9.1 \%$ ) and fewer permanent ileostomies ( 0 vs $1.0 \%$ ).

\section{Conclusion}

Primary resection for patients with acute RSCO seems to be associated with higher mortality and major morbidity rates than stent placement and elective resection. In addition, stent placement resulted in fewer anastomotic leakages and permanent ileostomies. However, as no high level studies are available on the optimal treatment of RSCO and proximal stenting is considered technically challenging, future comparative studies are warranted for the development of an evidence based clinical decision guideline. 


\section{INTRODUCTION}

Approximately $15 \%$ of patients with colon cancer present with colonic obstruction as a first symptom. ${ }^{1}$ This potentially life-threatening condition generally requires emergency intervention, and $32.5-54 \%$ of emergency surgeries for colonic obstruction are performed for tumours located in the proximal colon. ${ }^{2,3}$ Traditionally, malignant obstruction of the right colon has been treated with resection and primary anastomosis, which was deemed safe after prospective data showed no difference in mortality between emergency and elective surgery for right-sided colonic obstructions. ${ }^{4}$ However, recent studies found significantly higher operative risks for emergency resection compared to elective surgery. ${ }^{5,6}$

Indeed, patients presenting with right-sided colonic obstruction (RSCO) are generally older and have a more advanced tumour stage than electively treated patients. ${ }^{3}$ Added to this poor general condition, patients commonly have a distended bowel and often a period of reduced intake and weight loss, which likely will lead to high mortality and morbidity rates following emergency surgery. This prompted the search for valid alternatives for these frail patients, in whom the benefits of surgery might not outweigh the risks. Colonic stents can be used to address the immediate problem of a colonic obstruction and yet allow time for careful preparation for an extensive operation, thus, serving as a 'bridge to surgery'. This time can be used to optimise the patient's condition and allows for better staging, which could prevent unnecessary operations in inoperable patients. ${ }^{6}$ However, only limited data are available regarding stent placement in the proximal colon. Likewise, a staged approach with initial creation of a deviating ileostomy followed by elective tumour resection could theoretically be of benefit for patients who are in a poor preoperative condition at the time of presentation. This approach is not uncommon for left-sided colon obstruction, but very rarely used for rightsided obstructions.

Through a systematic review of available literature, this study aims to provide an overview of procedure-related mortality and morbidity rates of both primary resection and bridge to surgery with a colonic stent followed by elective resection in patients with acute malignant RSCO and to identify possible preoperative risk factors associated with worse outcomes after emergency intervention.

\section{METHODS}

This systematic review was conducted according to the Preferred Reporting Items for Systematic Reviews and Meta-Analysis (PRISMA) guidelines. ${ }^{7}$ Inclusion criteria for article selection were all studies containing information on treatment for acute right-sided colon obstruction.

\section{Search Strategy}

A systematic search was performed in MEDLINE (Pubmed), EMBASE (Ovid) and the Cochrane Database of Systematic Reviews. The search was restricted to publications in English and Dutch. 
Studies involving animal experiments were also excluded. The final search was performed on March 26, 2015. The Search strategy for Pubmed included colon obstruction[tiab] OR colonic obstruction[tiab] OR obstructed colon[tiab] OR malignant obstruction[tiab] OR proximal obstruction[tiab] OR right sided obstruction[tiab] OR colonic ileus[tiab] AND (surgery[tiab] OR colectomy[tiab] OR hemicolectomy[tiab] OR resection[tiab] OR colostomy[tiab] OR ostomy[tiab] OR stoma[tiab] OR stent[tiab] OR stents[tiab]). In addition, EMBASE and Cochrane databases were searched for relevant literature using the same search terms for domain and determinant. The reference lists of included articles were screened to identify additional studies.

\section{Study selection and quality assessment}

Two reviewers (FA and $\mathrm{HB}$ ) independently selected all relevant studies based on title and abstract. Studies were included when the location of obstruction was proximal, when the intervention was performed in an emergency setting and when data on post-intervention morbidity and/or mortality were provided. Conference abstracts without subsequent publication were excluded. In addition, studies in which outcome measures were not reported specifically for proximal obstruction or emergency intervention, $<10$ patients included, $<80 \%$ of the colonic obstruction caused by colon cancer or studies with a solely palliative approach were excluded from analysis. Both reviewers read the full text of the remaining articles. Final inclusion was based on consensus. All included studies were critically and independently appraised by both reviewers, using the MINORS criteria. ${ }^{8}$ Studies containing the following variables were included: age, gender, location of the obstruction, morbidity and mortality. For stent studies technical success, defined as deployment of the stent in the planned location, and clinical success defined as decompression leading to relief of symptoms, were also mandatory variables.

\section{Data Extraction}

Two reviewers (FA and $\mathrm{HB}$ ) extracted all data from the original articles. Baseline characteristics including age, gender, American Society of Anesthesiologists (ASA) score and location of the obstruction were obtained from included studies, as well as procedure-related mortality and morbidity. Mortality was defined as death within 30 days of the intervention or death within three months of the intervention without having been discharged from the hospital. Morbidity was subdivided in major and minor morbidity according to The Clavien-Dindo classification of surgical complications. ${ }^{9}$ Minor morbidity was defined as grade Illa or lower, major as grade IIIb or higher.

\section{Statistical Analysis}

Continuous variables were described as mean with range. Categorical variables were described as counts and percentages. Fisher's exact test was used for data analysis where necessary.

\section{RESULTS}

Study selection, study characteristics and methodological quality

In total, 1384 studies were identified by the search, and after removal of duplicates 1038 
FIGURE 1 | Flowchart of the search (march 2015)

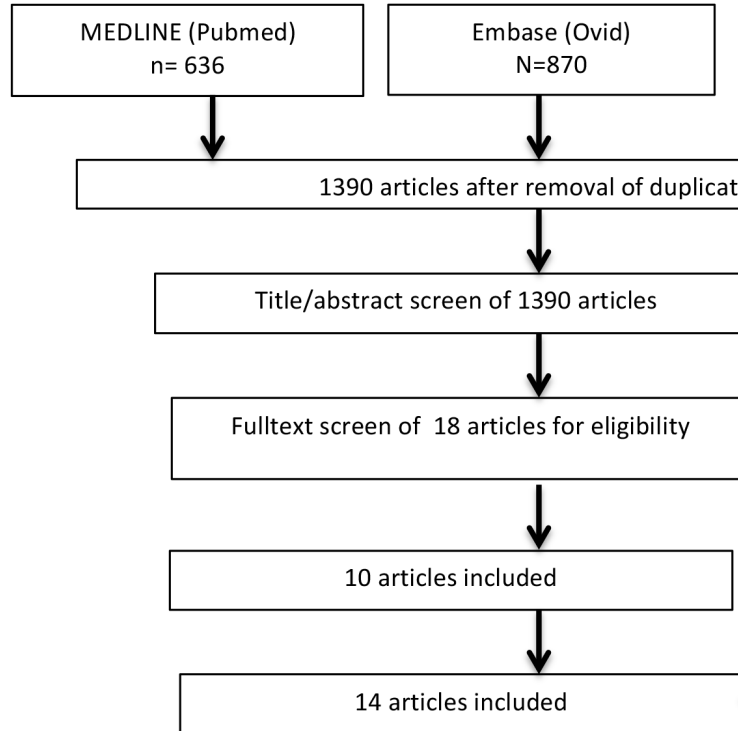

\section{Cochrane Database $\mathrm{N}=482$}
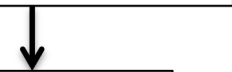

\section{tes}
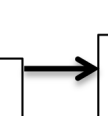

1372 articles excluded based on title or abstract screen

- No specfic report on RSCO outcomes $(n=5)$

$-<10$ patients $(n=2)$

- Overlapping patient

inclusion $(n=1)$

Cross-reference: +4 articles

remained. All articles were screened for eligibility based on title and abstract, leading to exclusion of 1020 studies. The remaining 18 studies were analysed in detail. Full text investigation led to exclusion of an additional eight studies. Five were excluded because they did not report outcomes for treatment of proximal obstruction specifically. Two were excluded because the number of patients with right-sided obstruction was very small ( $<10$ patients). One study was excluded because it included the same patients as another study; the excluded study had the smallest number of patients. Eventually, ten studies were included. Crossreference searching yielded another four articles relevant for this review. In total, 14 studies were included in this systematic review., , $^{3,10-12}$

Included articles were published between 2001 and 2013 and reported outcomes on a total of 2992 patients. Outcomes for primary resection were reported in ten articles (2837 patients) and for stent placement in four (155 patients). No studies reporting on deviating ileostomy creation as a treatment option were identified. All included studies are non-comparative cohort studies; ten describe retrospective data and the other four have a prospective design. The mean MINORS criteria score was 8.8 (range 6 to 11) out of a maximum possible score of 16 (table 1). Since no comparative studies were included, a meta-analysis of the data was not possible.

Details on demographics and patient characteristics are shown in table 2 and 3. For patients undergoing primary resection ( $n=2873)$, mean age was 63.1 ( 59 - 75 ) years, $48.8 \%$ were male, and $40.2 \%$ were ASA class III-IV. One hundred fifty five patients underwent stent placement; mean age was 68.4 (66.2 - 73) years, and 58.7\% were male. Unfortunately, ASA classification 
and tumour stage were not reported for patients undergoing stent placement. Of the 155 patients in the stent group, 95 patients had the stent placed as a bridge to surgery, in 60 patients stent placement was as a palliative approach (figure 2). All 95 patients, who received stent placement as a bridge to surgery, eventually underwent elective resection. Reasons for a palliative approach were the presence of metastatic disease in three studies, poor preoperative condition or metastatic disease in one, ${ }^{18}$ and not stated in one study. ${ }^{10,12,13}$

FIGURE 2 | Overview of treatment modalities performed in the included patients

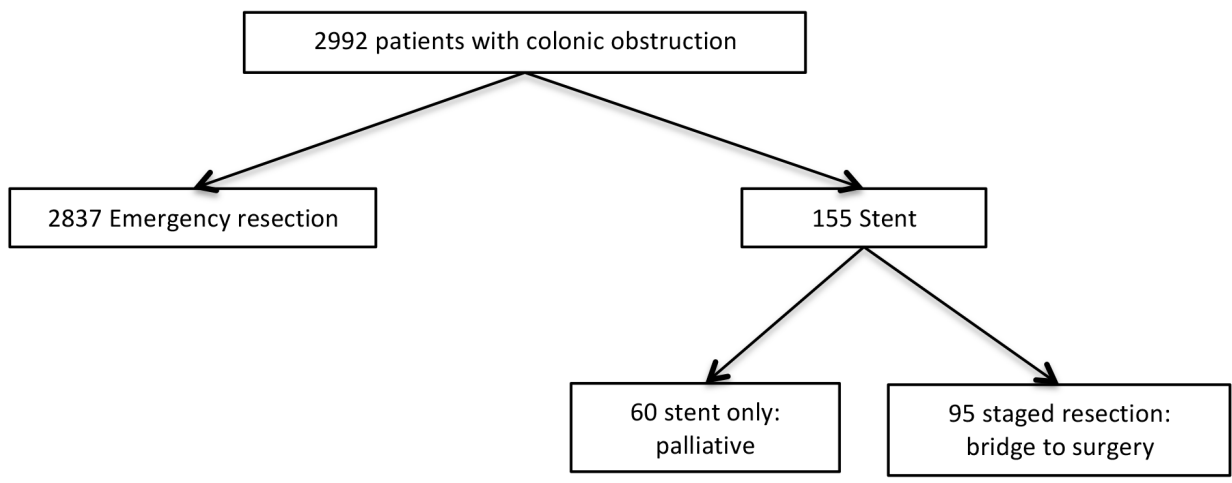

TABLE 1 | Critical appraisal using the MINORS criteria for selected papers

\begin{tabular}{|c|c|c|c|c|c|c|c|c|c|}
\hline Author, year & 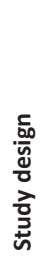 & $\begin{array}{l}\frac{\boldsymbol{E}}{\bar{\alpha}} \\
\frac{1}{\pi} \\
\frac{\Xi}{U}\end{array}$ & 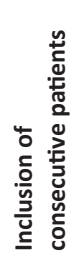 & 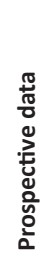 & 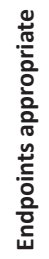 & 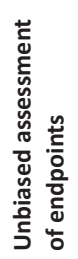 & 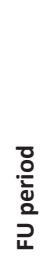 & 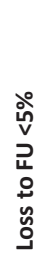 & 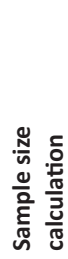 \\
\hline Aslar, $2010^{11}$ & $\mathrm{R}$ & 2 & 2 & 2 & 2 & 0 & 0 & 0 & 0 \\
\hline Chin, $2010^{12}$ & $P$ & 2 & 2 & 2 & 2 & 0 & 2 & 0 & 0 \\
\hline Cho, $2011^{13}$ & $R$ & 2 & 2 & 1 & 2 & 0 & 2 & 1 & 0 \\
\hline Dronamraju, $2009^{10}$ & $\mathrm{R}$ & 2 & 2 & 1 & 2 & 0 & 2 & 2 & 0 \\
\hline Frago, $2011^{14}$ & $\mathrm{R}$ & 2 & 2 & 1 & 2 & 0 & 2 & 2 & 0 \\
\hline Hsu, $2004^{15}$ & $\mathrm{R}$ & 2 & 2 & 1 & 1 & 0 & 0 & 0 & 0 \\
\hline Kobayashi, $2013^{5}$ & $P$ & 2 & 2 & 2 & 2 & 0 & 0 & 0 & 0 \\
\hline Lee, $2001^{16}$ & $\mathrm{R}$ & 2 & 2 & 1 & 2 & 0 & 2 & 0 & 0 \\
\hline $\mathrm{Ng}, 2007^{17}$ & $\mathrm{R}$ & 2 & 2 & 1 & 2 & 0 & 2 & 0 & 0 \\
\hline Sjo, $2009^{3}$ & $P$ & 2 & 2 & 2 & 2 & 0 & 0 & 0 & 0 \\
\hline Repici, $2007^{18}$ & $\mathrm{R}$ & 2 & 2 & 1 & 2 & 0 & 2 & 2 & 0 \\
\hline Tan, $2010^{19}$ & $R$ & 2 & 2 & 1 & 2 & 0 & 0 & 0 & 0 \\
\hline Tekkis, $2004^{20}$ & $P$ & 2 & 2 & 2 & 2 & 0 & 0 & 0 & 0 \\
\hline Yan, $2011^{21}$ & $\mathrm{R}$ & 2 & 2 & 1 & 2 & 0 & 0 & 0 & 0 \\
\hline
\end{tabular}

Abbreviations: $\mathrm{FU}=$ Follow-up, $\mathrm{P}=$ prospective, $\mathrm{R}=$ retrospective 


\section{Mortality}

All 14 studies reported on mortality rates (table 4 and 5).3,5,10-21 Mean overall mortality was $10.8 \%(8.1 \%-18.5 \%)$ in the primary resection group $(n=2873)$, and $0 \%$ in the stent group $(n=155)$. This difference was found to be statistically significant $(p=0.009)$.

\section{Morbidity}

Morbidity rates were reported in 11 out of 14 included studies.,10-15,17-19,21 Mean overall complication rate was $63.0 \%(22.2 \%-77.8 \%)$ for the primary resection group $(n=841)$, and $12.3 \%(3.8 \%-24.0 \%)$ for the stent group $(n=155)$. This difference was not statistically significant $(p=0.079)$, although borderline.

Morbidity rates could be classified further into major or minor morbidity in 8 of 14 studies ${ }^{11,14,15,17-19,21}$, reporting on 535 patients in the primary resection group and 155 in the stent group. Minor morbidity, grade IIla or lower, was not significantly different for the primary resection group $26.5 \%$ (16.3\% - 51.2\%) compared with the stent group 3.4\% (0\% - 7.1\%) ( $p=0.77)$. Major morbidity, grade IIIb or higher, was $23.9 \%(9.3 \%-35.6 \%)$ for the resection group, which is significantly higher than a $0.8 \%(0 \%-4.8 \%)$ major morbidity rate for the stent group $(p=0.049)$.

The occurrence of anastomotic leakage was reported in four studies, and was $9.1 \%$ (4.3\%$16.4 \%)$ following primary resection ( $n=465)$, and $0 \%$ for those patients in the stent group who subsequently underwent elective resection $(n=95) \cdot{ }^{11,14-16}$ All cases of stenting as a bridge to surgery to an operation were clinically successful. Median time from stent to operation was 19 days (range 7-42). No major surgical complications occurred and none required temporary stoma formation. 3 out of 95 patients (3.4\%) had minor morbidity after elective resection. ${ }^{11,14-16}$ Six studies reported on the creation of a protective ileostomy following primary resection. ${ }^{11,14-17,19}$ In total 38 patients $(7.6 \%, 0 \%-23.2 \%)$ were treated with an ileostomy, ultimately 4 patients $(1.0 \%, 0 \%-1.9 \%)$ ended up with a permanent ileostomy. No permanent ileostomies were created in the stent group.

Specific outcomes for the stent group are shown in table 5. Technical success was achieved in 95.5\% (86.0\%-100\%) of patients, and mean clinical success rate was $89.0 \%$ (78.0\% - 96.3\%). Stent related perforation occurred in $1.3 \%(0 \%-5 \%)$, stent migration in $2.0 \%(0 \%-8.0 \%)$ and stent re-occlusion in $3.2 \%(0 \%-11.0 \%)$ of patients. In addition to mortality and morbidity, we tried to identify preoperative risk factors for mortality. However, due to the lack of reporting on the different variables such as age, ASA-score and tumour location in the included studies, specific analysis was not possible. 

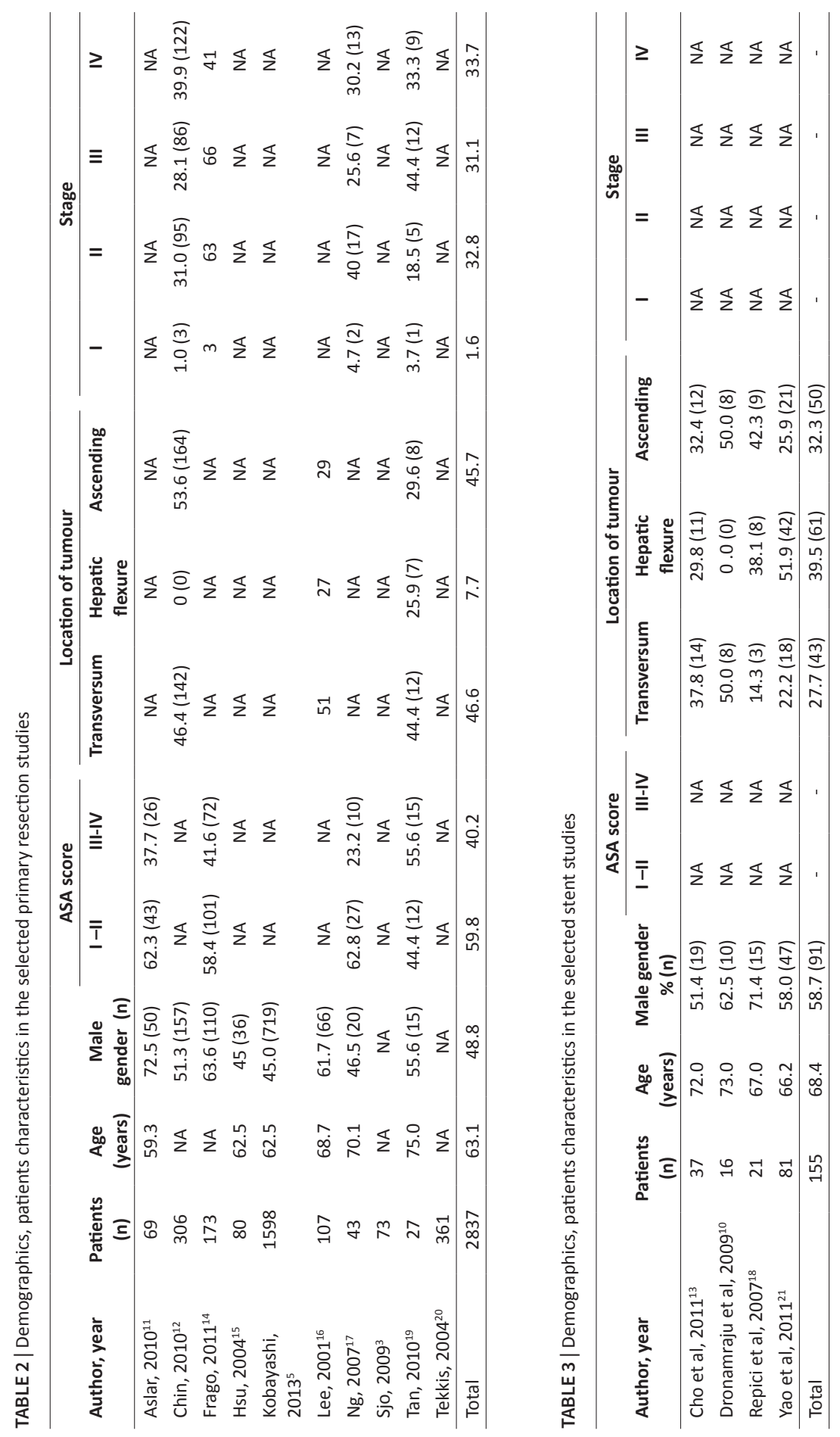

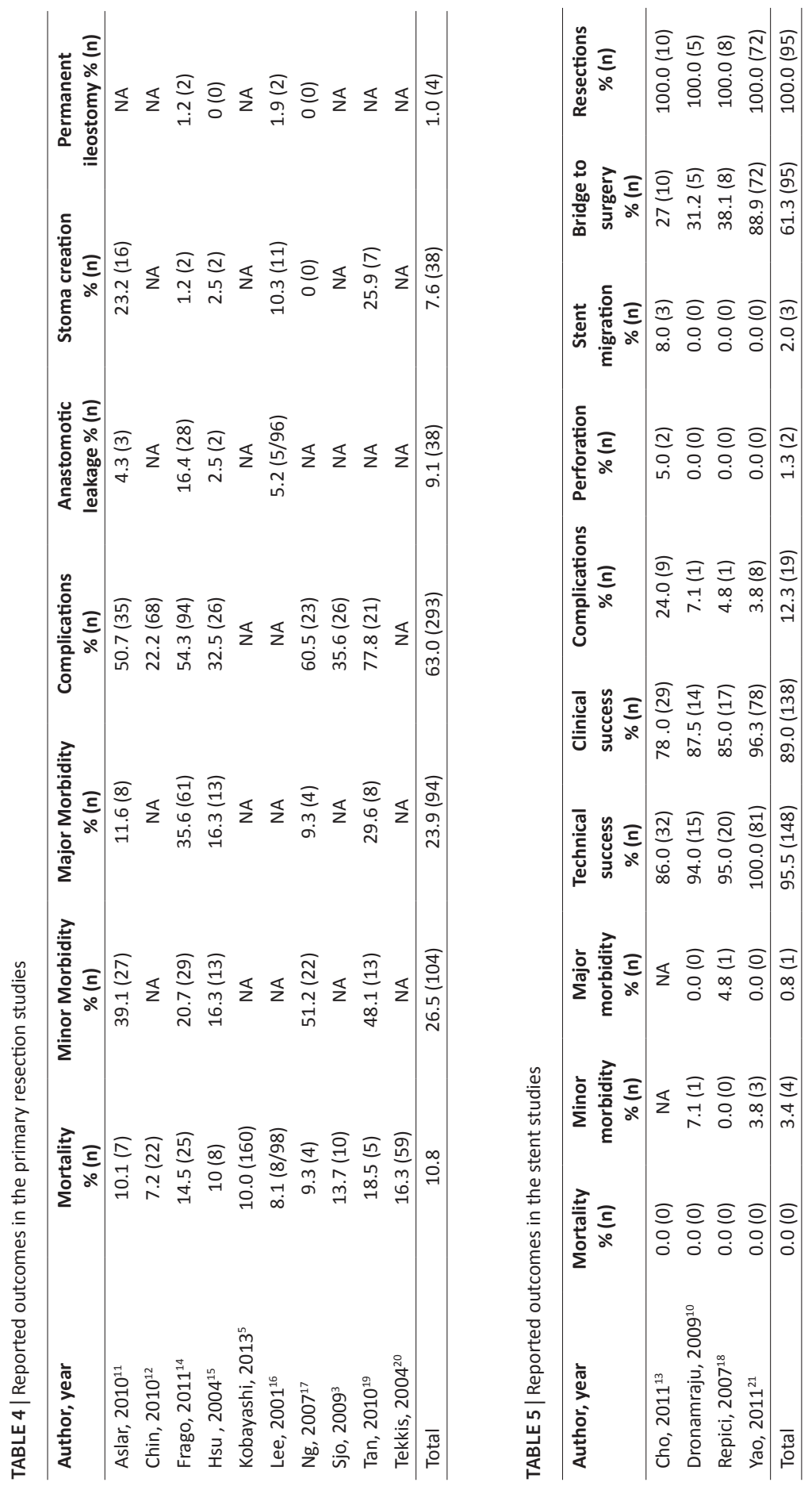


\section{DISCUSSION}

This systematic review was performed to provide an overview of the current literature on outcomes in patients with acute malignant right-sided colonic obstruction. To our knowledge, this is the first systematic review regarding treatment options specifically for acute malignant RSCO. The results suggest that a bridge to surgery approach using stent placement with subsequent elective resection is accompanied by significantly lower mortality and major morbidity when compared with primary resection ( $\mathrm{p}=0.009$ and $\mathrm{P}=0.049$ respectively). In addition, a tendency to a lower percentage of anastomotic leakage $(0.0 \%)$ and permanent ileostomy creation $(0.0 \%)$ in the patients treated with stent is demonstrated when compared to primary resection ( $9.1 \%$ and $1.0 \%$ respectively).

Primary resection and anastomosis is the current treatment of choice for acute RSCO, and the great majority (94.8\%) of patients included in this review received this treatment. The overall mortality of around $10 \%$ seems to be an improvement compared to $21 \%$ seen in older studies, ${ }^{24}$ and might be due to advances in critical care, antibiotic use and CT-guided abscess drainage. Nevertheless, mortality after emergency resection is much higher than after elective resection, which confirms that emergency right hemicolectomy is a high-risk procedure. ${ }^{2,3,5}$ This stresses the need for alternative treatment strategies leading to bowel decompression and preventing perforation before definitive treatment of the obstructing tumour.

Stenting as a bridge to surgery has been proposed as an attractive alternative to primary resection. ${ }^{27}$ In this review, clinical success of stent placement, with decompression of the colon and relief of symptoms was achieved in $89.0 \%$ (78.0 - 96.3\%), and perforation was found in $1.3 \%(0-5 \%)$ of patients. These observations are similar to those found in retrospective studies of stenting for left-sided colonic obstructions. ${ }^{25,26,27}$ However, prospective studies have shown clinical success rates of only $40.0-71.7 \%$ and perforation rates as high as $19-34 \%$ in left-sided obstructions. ${ }^{28-30}$ The discrepancy between retrospective and prospective data has been attributed to selection bias. Attempts for stent placement were generally not undertaken in patients with total obstructions in retrospective studies, because complete obstruction makes deployment technically difficult and increases the risk of perforations. ${ }^{31}$ Perforation is a feared complication, since it is associated with higher risks of mortality. Additionally, there are concerns about tumour spread and worsened long-term survival after perforation. ${ }^{29}$ Most studies reporting on stent placement are performed in left sided obstructions, less than $4 \%$ of the reported cases involve stent placement in the proximal colon. ${ }^{32}$ It is important to realize that stent placement in the right side of the colon is technically challenging. ${ }^{13,33,34}$

Despite the usual technical difficulty of proximal stent placement, technical and clinical success rates in all included retrospective studies are as high as $95.5 \%$ and $89.0 \%$, respectively. We tried to examine whether this might be due to patient selection. In the stent group, $55.2 \%$ had a subtotal obstruction, making stent placement easier. However, no data are available on the number of (sub)total obstructions in the acute resection group, making it impossible to 
determine whether patient selection occurred. In addition, we identified by whom the stents were placed, since experience has been shown to be important and determines technical and clinical success in stent placement. ${ }^{26}$ Only Dronamraju et al. and Repici et al. reported on this and in these two studies the stents were placed by a single endoscopist or gastroenterologist in each institution. How many stents they placed annually or their initial experience with stents was not stated. ${ }^{10,18}$ In $50 \%$ of the patients a Wallstent was used, $40.5 \%$ received a Hanaro stent and $9.5 \%$ a Bona stent. The diameter of these stents ranged from $20-24 \mathrm{~mm}$ and the length from $6-16 \mathrm{~cm}$. Whether these different stent designs or measures influenced the outcomes is unknown. Finally, tumour location did differ between the acute resection group and the stent group, i.e. $39.5 \%$ had the tumour located at the hepatic flexure in the acute resection group versus $7.7 \%$ in the stent group. However, tumour location is not a know risk factor for complications in colonic stent placement. ${ }^{26}$

We tried to identify risk factors associated with a higher mortality and morbidity following surgery. Identification of these risk factors might help in clinical decision-making. However, the reported data were too inconsistent to allow for analysis. The individual findings in some of the included studies showed increasing mortality and morbidity with increasing age. ${ }^{3,11,20}$ However, in all studies except for the study by Aslar et al., these ratios were calculated for left-sided and right-sided resections in elective and emergency settings combined. Kobayashi et al. found an odds ratio of $3.99(2.209-7.208)$ for patients with ASA class IV+V and an odds ratio of $2.317(1.564-3.431)$ for patients with ASA class III in a cohort of 15,275 patients who underwent emergency or elective right hemicolectomy. ${ }^{5}$ In addition, multiple other studies, both retrospective and prospective, associated ASA class III or higher with increased risks of mortality and/or major morbidity. ${ }^{3,11,19,20,35}$ Advanced age and ASA class III or higher are therefore likely to be independent risk factors for mortality, and those patients may benefit most from other treatment alternatives.

An important question currently is whether stent placement might have a negative influence on the oncologic long-term results, due to tumour manipulation and/or micro-perforations. None of the included studies report on long-term outcomes. For left-sided colonic obstructions, stent placement is currently only recommended in palliative patients or as a bridge to surgery when the patient is deemed unfit for major surgery since no consensus has been reached on the influence of stent placement on survival and recurrence rates. ${ }^{36,37}$

A systematic review such as this has several limitations that should be taken into account. First, due to lack of high-level evidence on acute RSCO, none of the included studies were comparative or blinded and most included studies were retrospective, increasing the risk of possible bias and confounding. Furthermore, in only one out of the four studies reporting on stent placement more than half of the included patients received stent as a bridge to surgery. Second, morbidity appeared to have been assessed differently in the various studies included in this study. Therefore, all complications were scored according to the surgical complication classification according to Clavien and Dindo. ${ }^{9}$ Although this classification is validated, it 
remains liable to subjective bias. Finally, the included studies did not all report on the different outcome measures, resulting in varying numbers of group sizes for the different outcomes. To our knowledge, this is the first systematic review reporting on alternative treatment options for right-sided colonic obstructions. Taking into consideration that literature reporting on this subject is scarce, this systematic review shows that stent placement seems to lead to significantly lower mortality and major morbidity rates when compared to primary resection. In addition, a lower percentage of patients with anastomotic leakage and a permanent ileostomy after eventual resection were seen when first treated with stent placement. Furthermore, stent placement can also be used as a palliative modality and could prevent unnecessary large abdominal surgery when further oncologic staging shows inoperable disease. The favourable results reported after stenting as a bridge to surgery are an indication that this could be a viable alternative for primary acute resection. However, the concerns over technical and clinical success, stent-related complications and long-term oncologic outcomes should be taken into account. Therefore, stent placement as a bridge to surgery could be a viable alternative for acute resection in curable patients with a high operative risk. In addition, it should only be applied when executed by a skilled and experienced endoscopist. Keeping the limitations of this study in mind, it is time for a prospective, comparative study between primary resection and stenting for malignant RSCO which will aid future clinical decisionmaking and may offer a suitable treatment for the individual patient. 


\section{REFERENCES}

1. Phillips RK, Hittinger R, Fry JS, Fielding LP Malignant large bowel obstruction. BJS. 1985;72:296-302

2. McArdle CS, Hole DJ. Emergency presentation of colorectal cancer is associated with poor 5-year survival. BJS.2004;91:605-609

3. Sjo OH, Larsen S, Lunde OC, Nesbakken A. Short term outcome after emergency and elective surgery for colon cancer. Colorectal Dis.2009;11:733-739

4. Smithers BM, Theile DE, Cohen JR, Evans EB, Davis NC . Emergency right hemicolectomy in colon carcinoma: a prospective study. Aust N Z J Surg 1986;56:749-752

5. Kobayashi $\mathrm{H}$, Miyata $\mathrm{H}$, Gotoh $\mathrm{M}$, Baba H, Kimura W, Kitagawa $Y$, et al. Risk model for right hemicolectomy based on 19,070 Japanese patients in the National Clinical Database. J Gastroenterol. 2014;49(6):1047-55

6. Smothers L, Hynan L, Fleming J, Turnage R, Simmang C, Anthony T. Emergency surgery for colon carcinoma. Dis Colon Rectum 2003;46:24-30

7. Moher D, Liberati A, Tetzlaff J, Altman DG. Preferred reporting items for systematic reviews and metaanalyses: the PRISMA statement. J Clin Epidemiol 2009;62(10):1006-1012

8. Slim K, Nini E, Forestier D, Kwiatkowski F, Panis Y, Chipponi J. Methodological index for non-randomized studies (minors): development and validation of a new instrument. ANZ J Surg. 2009;73(9):712-6

9. Clavien PA, Barkun J, de Oliveira ML, Vauthey JN, Dindo $D$, Schulick RD, et al. The Clavien-Dindo classification of surgical complications: five-year experience. Ann Surg 2009;250(2):187-96

10. Dronamraju SS, Ramamurthy S, Kellu SB, Hayat M. Role of Self-Expanding Metallic Stents in the Management of Malignant Obstruction of the Proximal Colon. Dis Colon Rectum 2009;52; 1657-1661

11. Aslar AK, Özdemir S, Mahmoudi H, Kuzu MA. Analysis of 230 cases of emergent surgery for obstructing colon cancer - lessons learned. J Gastrointest Surg 2011;15:110-119

12. Chin CC, Wang JY, Changchien CR, Huang WS, Tang R. Carcinoma obstruction fo the proximal colon cancer and long-term prognosis - obstruction is a predictor of worse outcome in TNM stage II tumor. Int J Colorectal Dis 2010;25:817-822

13. Cho YK, Kim SW, Lee BI, Lee KM, Lim DH, Kim JS, et al. Clinical outcome of self-expandable metal stent placement in the management of malignant proximal colon obstruction. Gut Liver 2011;5:165-170
14. Frago R, Biondo S, Millan M, Kreisler E, Golda T, Fraccalvieri D. Differences between proximal and distal obstructing colonic cancer after curative surgery. Colorectal Dis 2011;13:e116-e122

15. Hsu TC. Comparison of one-stage resection and anastomosis of acute complete obstruction of left and right colon. Am J Surg 2005;189: 384-387

16. Lee YM, Law WL, Chu KW, Poon RT. Emergency surgery for obstructing colorectal cancers: a comparison between right-sided and left-sided lesions. J Am Coll Surg 2001;192:719-725

17. Ng SS, Lee JF, Yiu RY, Li JC, Leung WW, Leung KL. Emergency laparoscopic-assisted versus open right hemicolectomy for obstructing right-sided colonic carcinoma: a comparative study of short-term clinical outcomes. World J Surg 2008;32:454-458

18. Repici A, Adler DG, Gibbs CM, Malesci A, Preatoni P, Baron TH. Stenting of the proximal colon in patients with malignant large bowel obstruction: techniques and outcomes. Gastrointest Endosc. 2007;66(5):940-4

19. Tan KK, Sim R. Surgery for obstructed colorectal malignancy in an Asian population: predictors of morbidity and comparison between left- and right-sided cancers. J Gastrointest Surg 2010;14:295

20. Tekkis PP, Kinsman R, Thompson MR, Stamatakis JD. The association of coloproctology of Great Britain and Ireland Study of large bowel obstruction caused by colorectal cancer. Ann Surg 2004;240:76-81

21. Yao LQ, Zhong YS, Xu MD, Xu JM, Zhou PH, Cai XL. Selfexpanding metallic stents drainage for acute proximal colon obstruction. World J Gastroenterol 2011;17:33423346

22. Fielding LP, Stewart-Brown S, Blesovsky L. Large bowel obstruction caused by cancer: a prospective study. BMJ 1979;2:517-519

23. Huann-Sheng Wang, Jen-Kou Lin, Chih-Yuan Mou, TzuChen Lin, Wei-Shone Chen, Jeng-Kae Jiang, Shung-Haur Yang. Long-term prognosis of patients with obstructing carcinoma of the right colon. The American Journal of Surgery 2004;187 497-500

24. Miller FB, Nikolov NR, Garrison RN.Emergency right colon resection. Arch Surg 1987;122(3):339-343.

25. Watt AM, Faragher IG, Griffin TT, Rieger NA, Maddern GJ. Self-expanding metallic stents for relieving malignant colorectal obstruction: a systematic review. Ann Surg. 2007;246(1):24-30 
26. Small AJ, Coelho-Prabhu N, Baron TH. Endoscopic placement of self-expandable metal stents for malignant colonic obstruction: long-term outcomes and complication factors. Gastrointest Endosc. 2010;71(3):560-72

27. Tilney HS, Lovegrove RE, Purkayastha S, Sains PS, Weston-Petrides GK, Darzi AW, et al. Comparison of colonic stenting and open surgery for malignant large bowel obstruction. Surg Endosc 2007;21(2):225-33

28. Sebastian S, Johnston S, Geoghegan T, Torreggiani W, Buckley M. Pooled analysis of the effi cacy and safety of self-expanding metal stenting in malignant colorectal obstruction. Am J Gastroenterol 2004;99: 2051-57

29. Pirlet IA, Slim K, Kwiatkowski F, Michot F, Millat BL. Emergency preoperative stenting versus surgery for acute left-sided malignant colonic obstruction: a multicenter randomized controlled trial. Surg Endosc. 2011;25(6):1814-21

30. van Hooft JE, Bemelman WA, Oldenburg B, Marinelli AW, Holzik MF, Grubben MJ, Sprangers MA, Dijkgraaf MG, Fockens P, collaborative Dutch Stent-In study group. Colonic stenting versus emergency surgery for acute left-sided malignant colonic obstruction: a multicentre randomised trial.Lancet Oncol. 2011;12(4):344-52

31. Ansaloni L, Andersson RE, Bazzoli F, Catena F, Cennamo V, Di Saverio S, et al. Guidelines in the management of obstructing cancer of the left colon: consensus conference of the world society of emergency surgery (WSES) and peritoneum and surgery (PnS) society. World J Emerg Surg 2010;28;5:29
32. Khot UP, Lang AW, Murali K. Parker MC. Systematic review if the efficacy and safety of colorectal stents. BJS. 2010;89:1096-102

33. Bonin EA, Baron TH. Update on the Indications and Use of Colonic Stents. Curr Gastroenterol Rep. 2010;12(5):374-82

34. Keymling M. Colorectal stenting. Endoscopy. 2003;35(3):234-8

35. Tan KK, Liu JZ, Yeow Y, Gunasekaran S, Tan JJ. Is emergency right hemicolectomy still associated with significant morbidity and mortality rates? An institution's experience of 207 cases over 6 years. Int Colorectal Dis 2011;26:1157-1161

36. van Hooft JE, van Halsema EE, Vanbiervliet G, BeetsTan RG, DeWitt JM, Donnellan F, Dumonceau JM, Glynne-Jones RG, Hassan C, Jiménez-Perez J, Meisner S, Muthusamy VR, Parker MC, Regimbeau JM, Sabbagh C, Sagar J, Tanis PJ, Vandervoort J, Webster GJ, Manes G, Barthet MA, Repici A. Self-expandable metal stents for obstructing colonic and extracolonic cancer: European Society of Gastrointestinal Endoscopy (ESGE) Clinical Guideline. Gastrointest Endosc. 2014;80(5):747-61.e1-75

37. Erichsen R, Horváth-Puhó $\mathrm{E}$, Jacobsen JB, Nilsson $\mathrm{T}$, Baron JA, Sørensen HT. Long-term mortality and recurrence after colorectal cancer surgery with preoperative stenting: a Danish nationwide cohort study. Endoscopy. 2015;47(6):517-24 



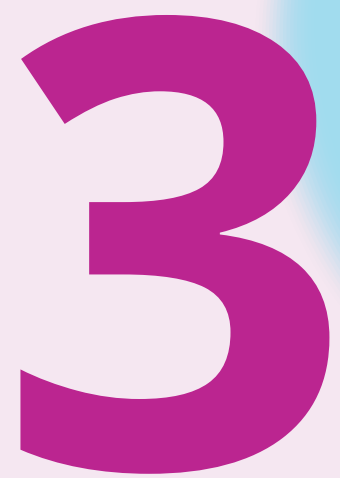

A population based analysis of three treatment modalities for malignant obstruction of the proximal colon: acute resection versus stent or stoma as a bridge to surgery

Annals of Surgical Oncology 2016

\author{
F.J. Amelung \\ E.C.J. Consten \\ P.D. Siersema \\ P.J. Tanis
}




\section{ABSTRACT}

\section{Background}

Malignant obstruction of the proximal colon (MOPC) has traditionally been treated with acute resection. However, morbidity and mortality rates following these emergency surgeries are high. Initial bowel decompression by stent placement or stoma construction has been used for distal obstructions as an alternative approach. This study aimed to evaluate whether these alternative treatment strategies could be beneficial for patients with a MOPC as well.

\section{Methods}

All patients undergoing a colonic resection for a MOPC between January 2009 and December 2013 and who were registered in the Dutch Surgical Colorectal Audit were analyzed.

\section{Results}

From the 49.013 patients registered in the DSCA, 1860 (3.8\%) were selected for further analysis. Acute resection was performed in 1774 patients (95.4\%), 44 patients (2.4\%) were treated with initial decompression using stent placement and resection and 42 patients $(2.3 \%)$ with stoma construction followed by resection. 30-day mortality was $8.8 \%, 2.4 \%$ and $2.4 \%$, respectively. Mortality was significantly lower after a bridging strategy (stent or stoma) compared to acute resection ( $p=0.04$ ). Complications following the resection occurred in $39.6 \%$ in the acute resection group and in $27.3 \%$ and $31.7 \%$ in the stent and stoma group, respectively $(p=0.167)$.

\section{Conclusion}

Acute resection was performed in the vast majority of patients with obstructive proximal colon cancer and resulted in a $40 \%$ morbidity and $9 \%$ mortality rates. A bridging strategy may be a valid alternative in some of these patients as a significantly lower postoperative mortality rate was seen in a subgroup of patients initially treated with a stent or stoma. 


\section{INTRODUCTION}

Colon carcinoma is one of the most frequently encountered malignancies in the western world and each year its incidence increases. ${ }^{1}$ Up to $9-13 \%$ of all patients with colon cancer present with acute bowel obstruction..$^{2-4}$ Approximately $33-54 \%$ of these obstructing tumors are located in the proximal colon..$^{5-7}$

Malignant obstruction of the proximal colon (MOPC) is considered a life threatening condition that requires emergency surgery. Traditionally, MOPC is treated with acute resection and primary anastomosis, which was deemed safe after several prospective studies showed no difference in mortality or morbidity rates when emergency and elective resections were compared. ${ }^{8}$ Recent studies, however, have shown significantly higher mortality rates in up to $34 \%$ of patients after emergency resections. ${ }^{9-11}$

Patients presenting with MOPC are often elderly and in a poor physical condition due to several days of reduced intake, vomiting and weight loss prior to presentation. These factors are associated with an increased operative risk leading to high mortality rates. ${ }^{9,11,12}$ Initial colonic decompression using a minimally invasive procedure as a bridge to surgery (BTS) might be an attractive alternative to acute resection. This approach creates time to optimize the patients' condition and perform oncologic staging, which could prevent unnecessary surgery in palliative patients. A BTS approach can be achieved by endoscopic stent placement at the site of obstruction or by stoma construction proximal to the obstruction.

The BTS approach has been extensively researched for left-sided colonic obstructions. In the recently published European Guideline on colonic stenting, BTS by stent placement in the curative setting is recommended for all patients $\geq 70$ years or with an ASA-score $\geq 3 .{ }^{13}$ However, less than $5 \%$ of all literature on colonic obstruction involves stenting in the proximal colon and, to our knowledge, no literature is available on stoma as BTS for MOPC at all. ${ }^{14} \mathrm{~A}$ recent systematic review comparing stent and acute resection for MOPC suggested lower mortality and morbidity rates when stent placement is used as BTS, but the included studies were small and of low quality. ${ }^{15}$

This study aimed to determine the use and corresponding outcomes of a BTS approach using stent placement or stoma creation in the Netherlands from 2009-2013, based on prospectively registered data. In addition, the outcomes of both BTS strategies were compared to the outcomes following emergency resection.

\section{METHODS}

\section{Study design and population}

Data of all patients who underwent a resection for MOPC between 2009-2013 were collected from a prospective national colorectal cancer registry: the Dutch Surgical Colorectal Audit (DSCA). This registry includes data of all patients undergoing resection of colorectal cancer in the 
Netherlands. All Dutch hospitals are obliged to deliver these data and validity is achieved by control tools in the web-based data entry program, by sending feedback on missing or improbable data, and by annual comparison with the National Cancer Registry on completeness and accuracy. ${ }^{16}$ The database was obtained after approval of the study protocol by the DSCA review board.

Patients were included for analysis when they met the following criteria: 1) symptomatic colonic obstruction, 2) proximal location of the obstruction (cecum, ascending colon, hepatic flexure or transverse colon) and 3) the obstruction was caused by histologically proven colon cancer. After patient selection from the DSCA database, patients were further subdivided into three groups depending on the initial treatment strategy applied; stent placement, stoma construction or acute resection. When initial decompression using stent placement had failed and emergency surgery was performed, the patient was still analyzed as having undergone stent placement. Patients presenting with perforation and fecal peritonitis were excluded from analyses.

\section{Data extraction}

The following data were extracted from the DSCA database: patient characteristics (age, gender, ASA-score), surgical characteristics (urgency of surgery, resection type, open/ laparoscopic approach, type of BTS approach used) data on the primary tumor (pathological TNM-stage, location), overall complication rate and mortality. Mortality was defined as death within 30 days or during hospital stay after resection. Overall complications were defined as surgical and non-surgical complications occurring within 30 days or in-hospital. No long-term data or data on complications associated with an initial decompression are registered in the DSCA. Furthermore, data on decision-making regarding treatment approaches is not available, although we know that mainly one regional teaching hospital performed stent as BTS during the study period. Since all data in the DSCA database are anonymous, retrieval of missing data was not possible.

\section{Outcome parameters}

Patients treated with stent placement, stoma construction or acute resection were compared on baseline characteristics and outcome parameters. The primary outcome measure was mortality. Other outcome parameters were overall complication rate and the percentage of radical resections.

\section{Statistical analysis}

Statistical analysis was performed using SPSS statistics 22. Continues variables were described as mean with standard deviation and range. Categorical variables were described as counts and percentages. Fisher's exact test or the $\mathrm{X}^{2}$-test was used for data analysis with categorical variables; one-way ANOVA for analysis of continues variables. Reported $p$-values are two-sided and were considered significant when $<0.05$. 


\section{RESULTS}

\section{Patient's characteristics}

Between January 2009 and December 201349.014 patients were included in the DSCA database. In total, 1860 patients had MOPC and were eligible for the present analysis. Overall, $1774(95.4 \%)$ patients were treated with acute resection, $44(2.4 \%)$ patients received a stent and subsequent resection, and 42 (2.3\%) patients had a stoma created as a BTS (Figure 1). A decrease in the frequency of stent placement was observed from approximately $3.5 \%$ in 2009 2012 to $0.5 \%$ in $2012-2013$.

ASA-score and gender were similar between treatment groups (Table 1). The stoma group had a significantly lower age (64.9 years) compared to the stent and acute resection groups (69.9 and 71.4 years, respectively, $p=0.001$ ). In addition, more tumors were localized in the transverse colon and fewer in the ascending colon in patients treated with stent compared to stoma or acute resection $(\mathrm{p}<0.001)$. Significantly more patients had a pT4 stage in the stoma group, while M1 stage was similar among treatment groups.

\section{Surgical characteristics}

After stent placement or stoma construction, resection was performed in an elective setting in $79.5 \%$ and $90.5 \%$ of the patients, respectively (Table 2 ). An emergency resection ( $<12$ hours) was performed in four patients of the stent group and in none of the stoma group. Reasons for emergency surgery, despite (an attempt to) colonic decompression, are not registered in the DSCA. However, two patients in the stent group had a registered perforation with fecal peritonitis as a possible explanation of the four emergency resections. Urgent resection (not according to elective planning) was performed in five and four patients of the stent and stoma groups, respectively.

The interval between the initial colonic decompression and eventual (elective) resection differed significantly between stent placement and stoma creation (28.1 vs. 109.9 days, $\mathrm{p}=0.01$ ). More transversectomies and left hemicolectomies were performed in the BTS groups compared to the acute resection group, which was related to differences in tumor localization. Furthermore, a laparoscopic approach was significantly more frequently used after stent placement when compared to stoma construction and acute resection ( $22.7 \%$ vs. $9.5 \%$ vs. $8.5 \%$, respectively, $p=0.027$ ). No differences were found in the total number of primary constructed anastomoses, number of protective stomas after resection or the use of postoperative chemotherapy.

\section{Outcome parameters}

Mortality in the stent, stoma and acute resection groups was $2.4 \%, 2.4 \%$ and $8.8 \%$, respectively. When stent and stoma patients were analyzed together as BTS-group and compared to acute resection, the difference in mortality was statistically significant $(p=0.04)$. Mortality rates for different subgroups based on age, ASA-score and type of resection, for patients that underwent acute resection, are shown in Table 3 . Subgroup analyses could not be performed for the stent and stoma groups due to the low number of events. 


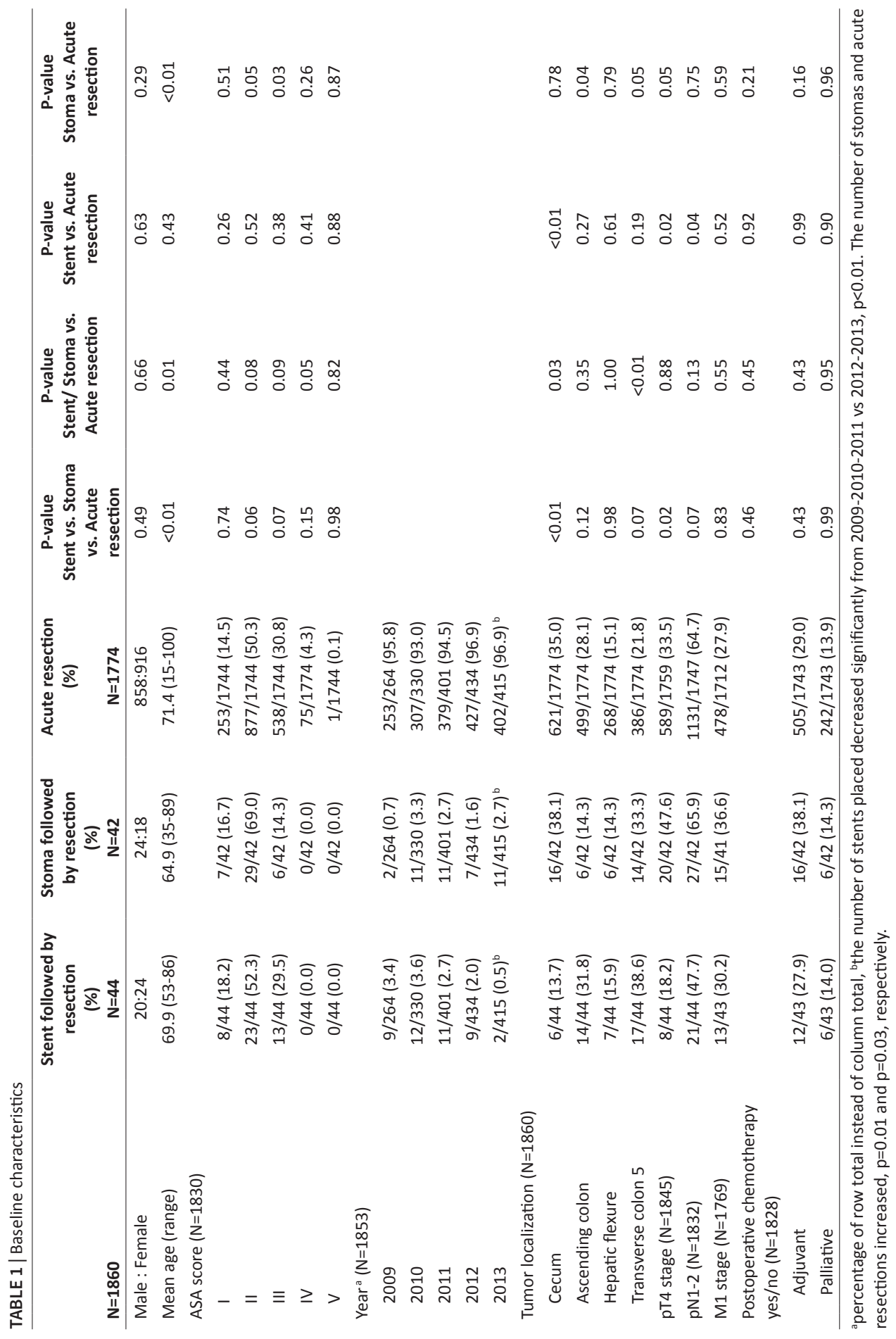




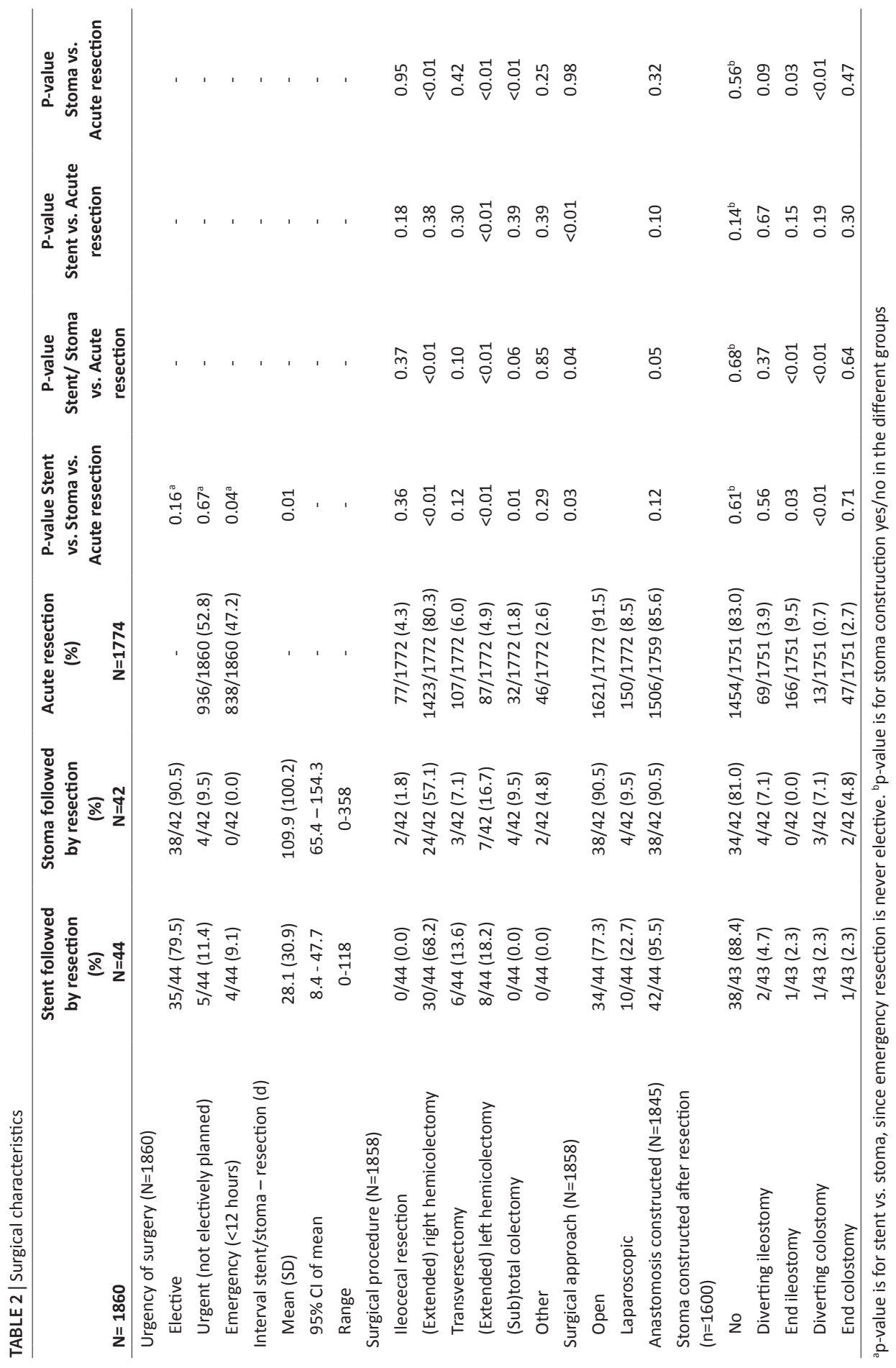




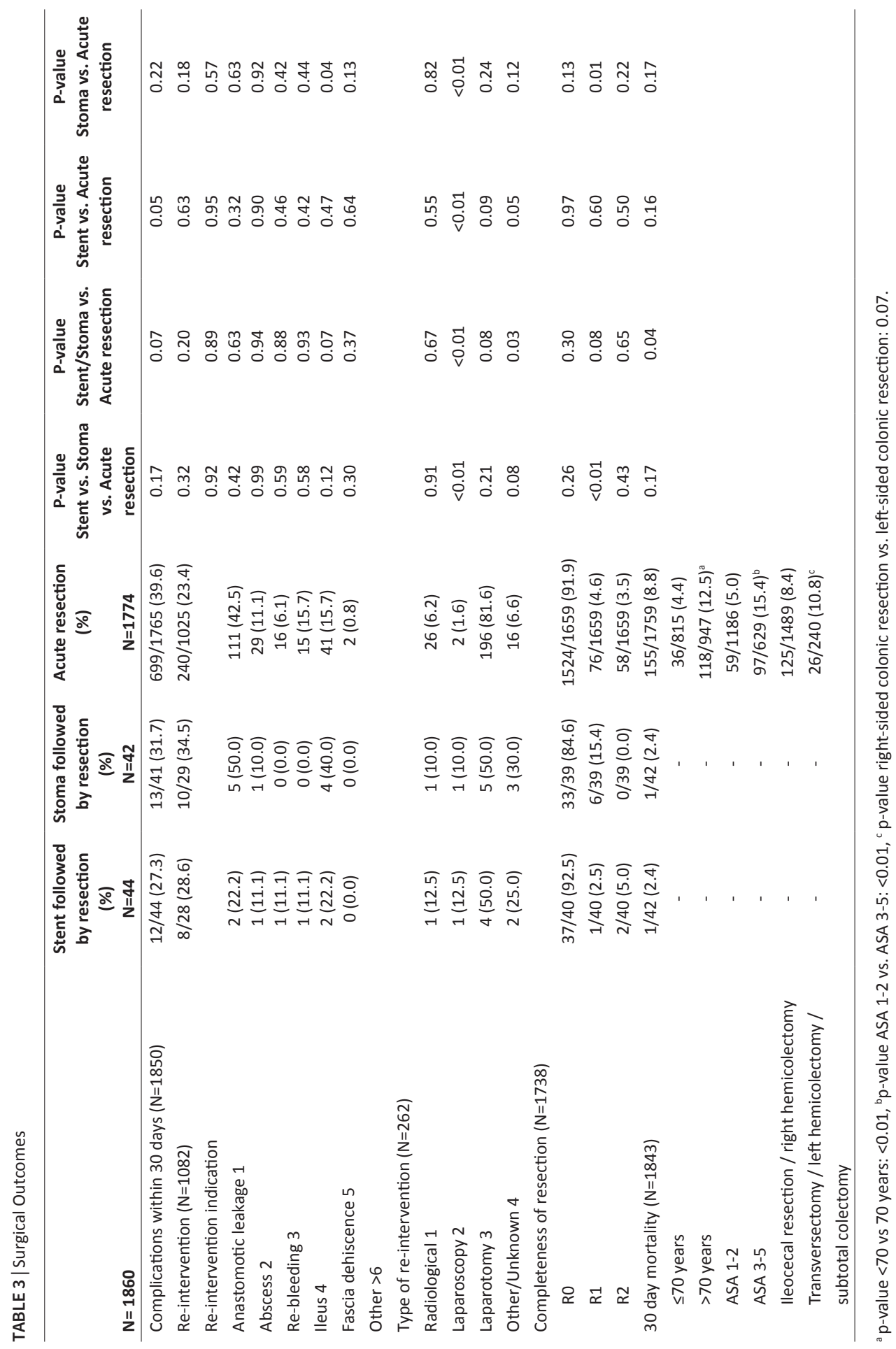


The number of complications within 30 days after resection was equal between treatment groups. However, if stent placement was compared separately to acute resection, a borderline significant difference in favor of stent was found ( $27.3 \%$ vs. $39.6 \%, p=0.051)$ (Table 3 ). Reintervention rate was not significantly different between the treatment groups; in addition, the type of complication requiring re-intervention did not differ. Postoperative complication rate for both the stent and stoma groups were lower when initial decompression was clinically successful and followed by elective resection. When acute resection was compared to only those patients in whom decompression with stent or stoma prior to resection was successful, complication rates did significantly differ (39.7\% after acute resection, $27.0 \%$ after stoma and elective resection and $22.9 \%$ for stent and elective resection, $p=0.043$ ).

No significant difference was found between treatment groups with regard to completeness of resection, however, more microscopic irradical resections (R1) were seen in the stoma group (15.4\%) compared to the stent $(2.5 \%)$ and acute resection (4.6\%) group.

\section{DISCUSSION}

This large population-based analysis of MOPC demonstrated that acute resection was performed in $95 \%$ of the patients, with a primary anastomosis rate of $86 \%$. A decompressing intervention as bridge to surgery was performed in only $5 \%$ of the patients. Mortality was significantly lower after a bridging strategy compared to acute resection. In addition, mortality rates after acute resection were approximately three times higher in patients $\geq 70$ years or with an ASA-score $\geq 3$.

These observations are clinically important since they may lead to a more patient-tailored treatment strategy. Our findings suggest that mortality rates could have been lower if more patients had been treated with a BTS, especially elderly patients with one or more comorbidities. Interestingly, a decrease in stent placement for MOPC from 3.5\% in 2009 to $0.5 \%$ in 2013 was seen. This change in decision-making is probably the result of the premature closure of two Dutch randomized controlled trials comparing stent with acute resection. Both trials were prematurely closed due to the high incidence of stent-related complications, making physicians more hesitant towards stent placement. ${ }^{17,18}$ Unfortunately, definitive conclusions cannot be drawn solely based on the outcomes of the present study since this is an observational cohort study with a risk of selection bias. It is not known from the DSCA database how patients were selected for the different treatment strategies. 92 hospitals included patients and treatment approaches highly depend on local expertise. Despite these methodological issues, the present data represent the best available evidence so far, since no randomized trials have been performed and the present study is the largest comparative series available.

The current findings are in line with observations in left-sided colonic obstructions (LSCO) in the literature. ${ }^{19}$ It may well be that treatment strategies for MOPC and LSCO should be identical. 
Nonetheless, the recently published European guideline on colonic stenting recommends a bridging strategy only for LSCO patients with an increased operative risk (age $\geq 70$ years/ASAscore $\geq 3) \cdot{ }^{13}$ The present data support such an approach for MOPC as well, which could ultimately lower mortality rates, especially in elderly and frail patients.

Several studies have tried to identify independent predictors of mortality when performing emergency surgery for large bowel obstruction. Tekkis et al. analyzed a group of right- and left-sided colonic obstructions and found significantly higher mortality for patients $\geq 70$ years or with an ASA-score $\geq 3 .{ }^{20}$ Kobayashi et al. evaluated a cohort of 15.275 patients who underwent a right hemicolectomy and found an odds ratio of 2.32 in patients with ASA-scores $\geq 3 .{ }^{9}$ In addition, several other studies have associated ASA-score $\geq 3$ and advanced age with higher mortality after colorectal surgery; however, no studies have specifically identified risk factors for MOPC. ${ }^{11,21,22}$

Studies on MOPC are scarce and to our knowledge no other articles on decompressing stoma as BTS for MOPC have been published. A recent systematic review comparing stent as BTS with acute resection in MOPC patients showed a mortality rate of $0 \%$ in the stent group versus $10.8 \%$ in the acute resection group ( $p=0.009)$. In addition, stenting was associated with lower morbidity rates and fewer permanent stomas. These retrospective results are similar and supportive to those found is our prospective analysis. ${ }^{15}$ However, these data should be interpreted with caution since all included studies in the systematic review were cohort studies. Kye et al. (2016) recently published the first study directly comparing stent and acute resection as treatment options for MOPC. Similar to our outcomes, they found laparoscopic resection rate to be significantly higher following stent placement. In addition, significantly more lymph nodes were harvested in the stent group. In contrary to our study, however, they did not find a significant difference in 30-day mortality and morbidity. ${ }^{23} \mathrm{~A}$ possible explanation for this discrepancy could be that they included considerable fewer patients than the current study and might have lacked power to demonstrate a significant difference.

Based on the currently available data, a bridging strategy might be the preferred initial approach in elderly and/or frail patients, where the risk of emergency surgery might be relatively high. However, stent placement for LSCO has been used with reserve during the past years, which is probably due to fear about stent-related complications and uncertainty about the oncologic long-term outcomes. ${ }^{24,25}$ Initially, based on retrospective data, stent placement for LSCO was thought to be a promising alternative to acute resection. However, several prospective trials had to be closed due to stent-related complications. ${ }^{17,26}$ The high technical and clinical success rates in retrospective studies might have been due to selection bias, where only patients with a subtotal, instead of a total occlusion were treated with stent placement. It is important to realize that this could also be the case for MOPC, since no prospective data are available yet. In addition, the long distance from the anus and the tortuosity of the bowel make proximal stenting considerably more difficult than in the distal colon. ${ }^{27-29}$ In line with this, higher technical failure rates have been reported for proximal stenting, which is most 
commonly caused by an inability to pass the guidewire trough an angulated colon, such as the hepatic flexure. ${ }^{29}$

The DSCA database has a high participation rate from Dutch hospitals (>95\%) and presents a good reflection of general practice in surgery for colorectal cancer in the Netherlands. ${ }^{1} \mathrm{~A}$ few limitations should be kept in mind. DSCA data are registered anonymously, making it impossible to retrieve missing values from the original patient files. Furthermore, since only patients with a colorectal resection are included in the DSCA database, patients in whom a stent was placed but never underwent colonic resection were excluded. Another limitation is that complications and hospital stay related to an initial decompression are not available in the registry; this might have had a positive influence on the overall outcomes in the BTS treatment groups.

Keeping these considerations in mind, this study indicates a possible advantage for stent or stoma as a bridge to surgery in patients with MOPC compared to acute resection, and high-risk patients potentially benefit most from such a strategy. Our data suggest that the current recommendation for stenting in left-sided colon cancer (ESGE guideline ${ }^{13}$ ) can be extended to proximal obstructions. A decompressing stoma can be considered an alternative in patients with high operative risk if stenting is not technically feasible, or in locally advanced tumors. To optimize a patient-tailored treatment strategy, future research should be focused on identifying more predictors to enable better selection of subgroups of patients who benefit most from a specific treatment strategy. 


\section{REFERENCES}

1. Jemal A, Bray F, Center MM, Ferlay J, Ward E, Forman D. Global cancer statistics. CA Cancer J Clin 2011;61:6990

2. Jullumstro E, Wibe A, Lydersen S, Edna TH. Colon cancer incidence, presentation, treatment and outcomes over 25 years. Colorectal Dis 2011;13:512-8

3. Cheynel N, Cortet M, Lepage C, Benoit L, Faivre J, Bouvier AM. Trends in frequency and management of obstructing colorectal cancers in a well-defined population. Dis Colon Rectum 2007;50:1568-75

4. Winner M, Mooney SJ, Hershman DL et al. Incidence and predictors of bowel obstruction in elderly patients with stage IV colon cancer: a population-based cohort study. J Am Med Assoc Surg 2013;148:715-22

5. Lee YM, Law WL, Chu KW, Poon RT. Emergency surgery for obstructing colorectal cancers: a comparison between right-sided and left-sided lesions. J Am Coll Surg. 2001;192(6):719-25

6. Hsu TC.Comparison of one-stage resection and anastomosis of acute complete obstruction of left and right colon. Am J Surg. 2005;189(4):384-7

7. McArdle CS, Hole DJ. Emergency presentation of colorectal cancer is associated with poor 5-year survival. Br J Surg. 2004;91(5):605-9

8. Smithers BM, Theile DE, Cohen JR, Evans EB, Davis NC. Emergency right hemicolectomy in colon carcinoma: a prospective study. Aust N Z J Surg 1986;56:749-752

9. Kobayashi $\mathrm{H}$, Miyata $\mathrm{H}$, Gotoh $\mathrm{M}$ et al. Risk model for right hemicolectomy based on 19,070 Japanese patients in the National Clinical Database. J Gastroenterol. 2014;49(6):1047-55

10. Smothers L, Hynan L, Fleming J, Turnage R, Simmang C, Anthony T. Emergency surgery for colon carcinoma. Dis Colon Rectum 2003;46:24-30

11. Sjo OH, Larsen S, Lunde OC, Nesbakken A. Short term outcome after emergency and elective surgery for colon cancer. Colorectal Dis. 2009;11(7):733-9

12. Tan KK, Sim R. Surgery for obstructed colorectal malignancy in an Asian population: predictors of morbidity and comparison between left- and right-sided cancers. J Gastrointest Surg 2010;14:295

13. van Hooft JE, van Halsema EE, Vanbiervliet $G$ etal. Selfexpandable metal stents for obstructing colonic and extracolonic cancer: European Society of Gastrointestinal Endoscopy (ESGE) Clinical Guideline. Gastrointest Endosc. 2014;80(5):747-61.e1-75
14. Bonin EA, Baron TH. Update on the Indications and Use of Colonic Stents. Curr Gastroenterol Rep 2010;12(5):374-382

15. Amelung FJ, de Beaufort HW, Siersema PD, Verheijen PM, Consten ECJ. Emergency resection versus bridge to surgery with stenting in patients with acute right-sided colonic obstruction: a systematic review focusing on mortality and morbidity rates. Int J Colorectal Dis. 2015;30(9):1147-55

16. Van Leersum NJ, Snijders HS, Henneman D et al. The Dutch surgical colorectal audit. Eur J Surg Oncol 2013;39:1063-1070

17. van Hooft JE, Bemelman WA, Oldenburg B et al. Colonic stenting versus emergency surgery for acute left-sided malignant colonic obstruction: a multicentre randomised trial. Lancet Oncol 2011; 12:344-352

18. Van Hooft JE, Fockend P, Marinelli AW, Timmer R, van Berkel AM, Bossuyt PM, Bemelman WA. Early closure of a multicenter randomized clinical trial of endoscopic stenting versus surgery for stage IV left-sided colorectal cancer. Endoscopy 2008; 40: 184 - 191

19. Tanis PJ, Paulino Pereira NR, van Hooft JE, Consten EC, Bemelman WA. Resection of Obstructive Left-Sided Colon Cancer at a National Level: A Prospective Analysis of Short-Term Outcomes in 1,816 Patients. Dig Surg. 2015;4;32(5):317-324

20. Tekkis PP, Kinsman R, Thompson MR, Stamatakis JD. The association of coloproctology of Great Britain and Ireland Study of large bowel obstruction caused by colorectal cancer. Ann Surg 2004;240:76-81

21. Aslar AK, Özdemir S, Mahmoudi H, Kuzu MA. Analysis of 230 cases of emergent surgery for obstructing colon cancer - lessons learned. J Gastrointest Surg 2011;15:110-119

22. Tan KK, Sim R. Surgery for obstructed colorectal malignancy in an Asian population: predictors of morbidity and comparison between left- and right-sided cancers. J Gastrointest Surg 2010;14:295

23. Kye B H, Lee YS, Cho HM et al. Comparison of long-term outcomes between emergency surgery and bridge to surgery for obstruction in right sided colon cancer: a multicenter retrospective study. Ann Surg Oncol 2016;23(6):1867-74

24. Sloothaak DA, van den Berg MW, Dijkgraaf MG, Fockens P, Tanis PJ, van Hooft JE, Bemelman WA. Oncological outcome of malignant colonic obstruction in the Dutch Stent-In 2 trial. collaborative Dutch Stent-In study group. Br J Surg. 2014;101(13):1751-7 
25. Sabbagh C, Browet F, Diouf M et al. Is Stenting as "a Bridge to Surgery" an Oncologically Safe Strategy for the Management of Acute, Left-Sided, Malignant, Colonic Obstruction? Ann Surg. 2013;258(1):107-15

26. Pirlet IA, Slim K, Kwiatkowski F, Michot F, Millat BL. Emergency preoperative stenting versus surgery for acute left-sided malignant colonic obstruction: a multicenter randomized controlled trial. Surg Endosc. 2011;25(6):1814-21

27. Y. Saida, J. Nagao, Y. Nakamura, T. Enomoto, Y. Sumiyama, and K. Tominaga. Trans-anal decompression for colorectal obstruction. Gastroenterological Endoscopy, 2008;50;1;80-90

28. Bonin EA, Baron TH. Update on the Indications and Use of Colonic Stents. Curr Gastroenterol Rep. 2010;12(5):374-82

29. Cho YK, Kim SW, Lee BI et al. Clinical outcome of selfexpandable metal stent placement in the management of malignant proximal colon obstruction. Gut Liver 2011;5:165-170 



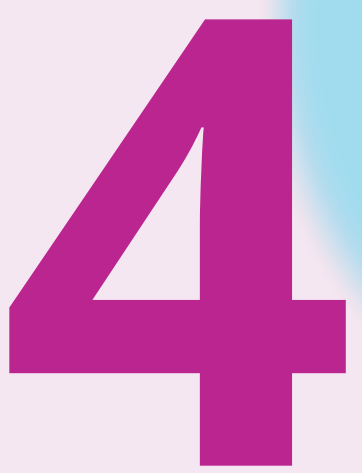

Self-expandable metal stent placement versus emergency resection for malignant proximal colon obstructions

Surgical Endoscopy 2017

F.J. Amelung

W.A. Draaisma

E.C.J. Consten

P.D. Siersema

F. ter Borg 


\section{ABSTRACT}

\section{Background}

Traditionally, all patients with a malignant obstruction of the proximal colon (MOPC) are treated with emergency resection. However, recent data suggest that Self-Expandable Metallic Stent (SEMS) placement could lower mortality and morbidity rates. This study therefore aimed to compare SEMS placement with emergency resection as treatment options for MOPC.

\section{Methods}

All consecutive patients that underwent SEMS placement for MOPC in the period 2004-2015 at our institution were identified. SEMS placement was the standard of care for colonic obstructions at our institution in that period. All included SEMS patients were matched (1:4) on age ( \pm 5 years), gender, ASA-score, tumor location, surgical approach and pTNM-stage with patients treated by emergency resection. Controls were selected from a national database that prospectively registers all patients undergoing surgery for colorectal cancer in the Netherlands.

\section{Results}

In total, 41 patients received SEMS placement for MOPC. In 19 patients SEMS served as a definite palliative measure and in 22 as bridge to surgery. Technical and clinical success rates of SEMS placement were $92.7 \%$ and $90.2 \%$, respectively. No significant differences between the SEMS and emergency resection group were found regarding morbidity and mortality rates, the number of radical resections and the number of primary anastomoses. Patients treated with SEMS were, however, less likely to have a temporary stoma constructed $(p=0.04)$. No SEMS-related complications occurred in patients in whom SEMS was placed as bridge to surgery, whereas one stent-related perforation, three stent migrations and five stent reobstructions were observed in the palliative group. Three re-obstructions could be treated with re-stenting, but all other SEMS-related complications required surgical intervention. In the palliative group, SEMS complications necessitating surgery occurred in $31.6 \%$ of the patients (6/19).

\section{Conclusion}

SEMS placement for MOPC appears to be a relatively feasible and safe alternative for emergency resection in both the curative and palliative setting. 


\section{INTRODUCTION}

Approximately $9-13 \%$ of the patients with colorectal carcinoma initially present with colonic obstruction, which is considered a life-threatening condition that requires immediate intervention. ${ }^{1,2}$ Current treatment strategies include emergency resection, self-expandable metal stent (SEMS) placement or deviating stoma construction. Both SEMS placement and stoma construction can be used as a bridge to elective surgery (BTS), hereby eliminating the need for major abdominal surgery in the emergency setting. ${ }^{3}$

A BTS approach is especially valuable in patients with an acute colonic obstruction, since they usually present in a poor clinical condition due to several days of reduced intake and weight loss prior to presentation. A BTS approach creates time to optimize the patients' condition before an elective resection is conducted. ${ }^{4,5}$

The BTS approach has been extensively studied for left-sided colonic obstructions (LSCO) and has become increasingly popular since it seems to be associated with lower morbidity and mortality rates compared to emergency resection. ${ }^{4-7}$ However, limited data is available on the safety and/or feasibility of this approach in malignant obstructions of the proximal colon (MOPC). Even though approximately $32-54 \%$ of the obstructing colon tumors are located in the proximal colon, it has been reported that only $5 \%$ of reported cases of colonic stenting involve the proximal colon..$^{8-11}$

Traditionally, MOPC is treated with emergency resection and primary anastomosis, which was was deemed safe after several studies showed similar morbidity and mortality rates when emergency and elective proximal resections were compared. ${ }^{12}$ However, recent studies have shown mortality rates following emergency resections to be significantly higher. ${ }^{13,14}$ This has prompted the search for a valid alternative and raised the question whether a BTS approach could also be beneficial in patients with MOPC.

Two previous studies show promising results for SEMS placement in MOPC, however, both studies are of limited methodological quality and definitive conclusions cannot be drawn based solely on the outcomes of these studies..$^{8-9}$ In the current study, we therefore assessed the safety of proximal stent placement and compared these data to case-matched patients that underwent emergency resection.

\section{MATERIALS AND METHODS}

\section{Study design and population}

Utilizing a case-control design, we matched all consecutive patients that underwent SEMS placement for acute MOPC between 2004 and 2015 at our institution to controls treated with emergency resection for MOPC. Matching occurred on a one-to-four basis using six covariates: gender, age ( \pm 5 year), ASA-score, tumor location, type of surgical procedure (open/laparoscopic) and pTNM-score. Patients were considered to have MOPC when the obstruction was: 1) symptomatic (absence of stool, abdominal pain, nausea and/or vomiting), 2) had a proximal 
location (cecum, ascending colon, hepatic flexure or transverse colon), 3) was confirmed with radiological imaging and 4) caused by histologically proven colon carcinoma.

A one-to-four match ratio was selected because statistical power is only marginally increased by adding more controls. ${ }^{15}$ When more than four matches were available, controls were randomly chosen. All controls were selected from a prospective national registry, the Dutch Surgical Colorectal Audit (DSCA). This registry includes all patients that undergo colonic resection for colorectal cancer in the Netherlands. It is obligatory for all Dutch hospitals to deliver these data to the database and validity is achieved by control tools in the web-based data entry program. ${ }^{16}$

\section{Outcome parameters}

Demographic, surgical and postoperative outcomes were collected from the patients' medical records in the SEMS group and extracted from the DSCA database for the emergency resection group. Demographic characteristics included age, gender, ASA-score, pTNM-stage, type of procedure performed, curative or palliative treatment intent and whether (neo-) adjuvant therapy was administered. The primary outcome measures were 30-day morbidity and mortality. Morbidity and mortality rates in the SEMS group included events following SEMS placement as well as elective resection. Complications were graded according to the classification of surgical complications created by Clavien et al.; major morbidity was classified as grade III or higher and minor morbidity as grade II or lower. ${ }^{17}$ Additional study outcomes were technical and clinical success rate of SEMS placement, primary anastomosis rate and temporary colostomy rate. Clinical success of SEMS placement was defined as the production of feces within 48 hours following SEMS placement.

In addition, in order to evaluate the safety of proximal stenting, all patients that had a SEMS placed for MOPC were compared to all patients that underwent distal stent placement at our institution during the same time period.

\section{SEMS placement and surgical technique}

All patients underwent either SEMS placement or emergency resection within 48 hours after presentation. Before SEMS placement, it would be decided whether the patient would be treated with curative or palliative intent based on CT-abdomen and chest-X-ray results. All SEMS were placed endoscopically by one of three endoscopists, experienced in colonic stenting. All three endoscopists had placed over 20 colonic stents before 2004, at the end op the study inclusion period the number of procedures performed per endoscopist ranged from 40 to 120 procedures. Placement of SEMS (Wallstent ${ }^{\circledR}$, Wallflex ${ }^{\circledR}$ (Boston Scientific) or Evolution ${ }^{\circledR}$ (Cook Medical)) took place under fluoroscopic control. SEMS length was based on the previously estimated length of the stenosis on CT. The malignant stricture was never passed with the endoscope or balloon dilated, since this is associated with an increased perforation risk. ${ }^{18,19}$

The stent was used as a palliative measure in case of incurable disease or high surgical risk $(A S A \geq 4)$. Patients with curable disease and acceptable surgical risk received SEMS placement 
as BTS. The elective resection included a routine mesocolic lymphadenectomy, with the goal of harvesting minimally 10 lymph nodes. The specific choice of resection was at the discretion of the treating surgeon and depended on tumor location and size.

\section{Statistical analysis}

Statistical analyses were performed using SPSS 23.0 (IBM). Continuous variables were tested for normality using the Kolmogorov-Smirnoff test; normally distributed variables were described as mean with standard deviation (SD), non-normally distributed variables as median with interquartile range (i.q.r.). Normally distributed variables were compared with the unpaired Student's $t$ test., non-normally distributed variables with the Mann-Whitney U test. Categorical variables were described as counts and percentages and were compared using Fisher's exact test. Kaplan-Meier analysis was used to investigate overall and disease free survival. A twosided $p$-value $<0.05$ was considered statistically significant.

\section{RESULTS}

\section{Patient characteristics and baseline}

Between 2004-2015, 41 patients presented with MOPC at our institution and all were treated with SEMS placement. Mean age was $74.8( \pm 10.7)$ years and 21 patients $(51.2 \%)$ were male. 30 patients (73.2\%) presented with TNM-stage III-IV and the location of the obstructing tumor was transverse colon in 15 patients (36.6\%), ascending colon in 15 patients (36.6\%), hepatic flexure in 6 patients $(14.6 \%)$ and cecum in 5 patients $(12.2 \%)$.

Since treatment algorithms differ for patients treated with palliative or curative intent, patients were divided into two groups for further analysis; 1 ) Patients treated with palliative intent (SEMS placement only, $n=19$ ) and 2) Patients treated with curative intent (SEMS placement as BTS followed by elective resection, $n=22$ ). (Figure 1 ) Indications for palliative treatment were inoperable disease $(n=12)$, high surgical risk $(n=6)$ or refusal of surgery $(n=1)$.

Matching of the 41 SEMS patients resulted in the inclusion of 164 patients treated with emergency resection; 76 were matched to the palliative SEMS group and 88 to the curative SEMS patients. As shown in Table 1, all baseline characteristics were similar between treatment groups with a $p$-value $>0.05$.

\section{SEMS-related outcomes}

In total, three different types of stents were placed: 29 Wallstents, 7 Wallflex stents and 5 Evolution stents. The type of stent choice was based on availability. SEMS placement was technically successful in 38 patients, resulting in an overall technical success rate of $92.7 \%$ (95\% Cl 84.7-100). Reasons for technical failure were the inability to pass the guidewire across the stricture $(n=2)$ or a synchronous colorectal tumor that precluded SEMS placement $(n=1)$. In addition, one patient in whom SEMS placement was technically successful did not experience relief of obstructive symptoms. All these patients had to undergo an emergency resection. Therefore, clinical success rate was $90.2 \%(95 \% \mathrm{Cl} 81.2-99.2)$. (Table 2) 
FIGURE 1 | Flowchart of patient selection

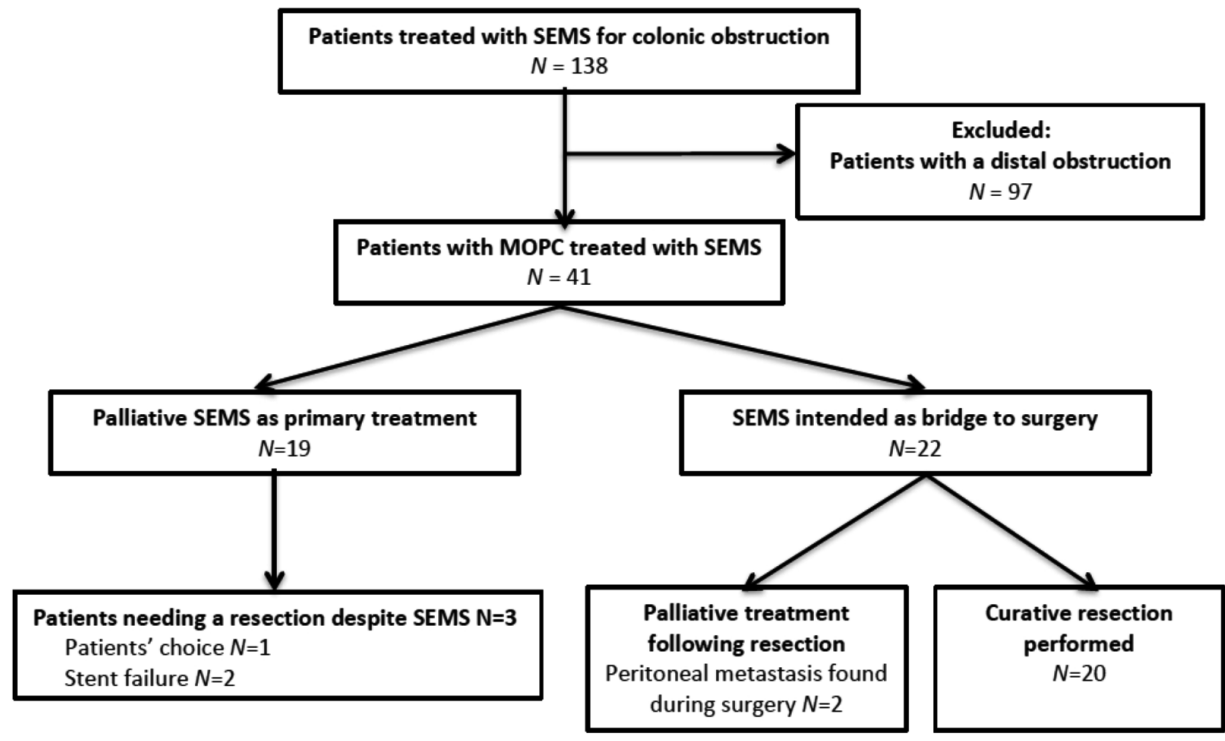

No SEMS-related complications occurred in patients in the BTS group. SEMS remained in place for a mean of $20( \pm 10.6)$ days and all successfully placed stents remained patent until the elective resection. However, in the palliative group, where SEMS was used as a long-term solution, five cases of stent re-obstruction (26.3\%), three cases of stent migration (15.8\%) and one stent-related perforation (5.3\%) were observed. The perforation occurred 1 year following SEMS placement and resulted in the patients' death (surgery was considered inappropriate given the extensive end-stage oncological situation). Stent re-obstructions occurred after 1 month ( $n=2), 6$ months $(n=1)$ and 7 months $(n=2)$. Three re-obstructions could be treated with re-stenting, all other SEMS-related complications required additional surgical interventions consisting of ileostomy construction $(n=1)$, ileocolonic bypass $(n=1)$ or resection with primary anastomosis $(n=3)$. Overall, this resulted in a long-term SEMS patency rate, defined as successful relief of obstructive symptoms by SEMS until death without the need of surgical interventions, of $68.4 \%(95 \% \mathrm{Cl} 57.6-99.2)$. Therefore stent placement spared approximately $70 \%$ of the palliative patients from major abdominal surgery.

\section{Comparison of SEMS and control group}

Mortality and morbidity

As shown in Table 3, no significant difference in mortality rate was found between patients treated with SEMS vs. emergency resection in both the curative and palliative groups $(p=1.00$ and $p=0.70$, respectively). In addition, major and minor morbidity were also found to be similar regardless of treatment approach. 
Primary anastomoses, temporary colostomies and the number of radical resections Primary anastomosis rate in the SEMS group was not significantly different from the emergency resection group in curatively treated patients $(100.0 \%$ vs. $90.9 \%, p=0.36)$. In the palliative group, $85.5 \%$ of the patients treated with emergency resection had a primary anastomosis constructed. In the palliative SEMS group only three patients eventually underwent a colonic resection and a primary anastomosis was constructed in all. Furthermore, SEMS placement led to a lower temporary stoma rate in both the curative as well as the palliative group ( $p=0.04$ and $p=0.04)$. The number of radical resections was similar between treatment groups.

\section{Hospital stay and time to resection}

The median total hospital stay was 9 (i.q.r. 4-18) days in the palliative SEMS group, and 11.5 (i.q.r. 10-15) days in the BTS group. Hospital stay included the total number of days the patient was admitted during SEMS placement and the elective resection. Hospital stay is not reported in the DSCA database and outcomes could therefore not be compared. The median time from SEMS placement to elective resection was 18.5 (i.q.r. 8.5-27.75) days.

\section{Overall and Disease free survival}

The median follow-up time was 4 months (i.q.r. 3-8, range 1-24) for the palliative and 36 months (i.q.r. 16-63, range 5-120) for the curative SEMS group. No long-term outcomes were available for the emergency resection group, since the DSCA database only includes short-term outcomes. Overall recurrence rate (including local recurrence and distant metastasis) in the curatively treated SEMS group was $36.4 \%(8 / 22)$. The median overall survival was 44 months (i.q.r. 16-63) and 4 months (i.q.r. 3-8) for the curative and palliative SEMS group, respectively (Figure 2). Three- and five-year survival rates were $63.6 \%$ and $50 \%$ in the curative SEMS group.

\section{Comparison between SEMS placement in the left- and right-colon}

In order to further evaluate the safety of proximal stenting, all patients that underwent proximal SEMS placement were compared to all consecutive patients that underwent distal SEMS placement at our hospital during the same time period (2004-2015). In total, 138 patients underwent SEMS placed for a colonic obstruction; 97 stents were placed in the distal colon and 41 in the proximal colon. Baseline characteristics between the two patient groups were comparable (Table 4). In addition, technical and clinical success rates of SEMS placement did not differ between both groups ( $92.7 \%$ vs. $95.9 \%, p=0.43$ and $90.2 \%$ vs. $82.3 \%, p=0.31$ ). Furthermore, 30-day stent-related short- and long-term complications, 30-day mortality and long-term SEMS success rate were similar (Table 4). 


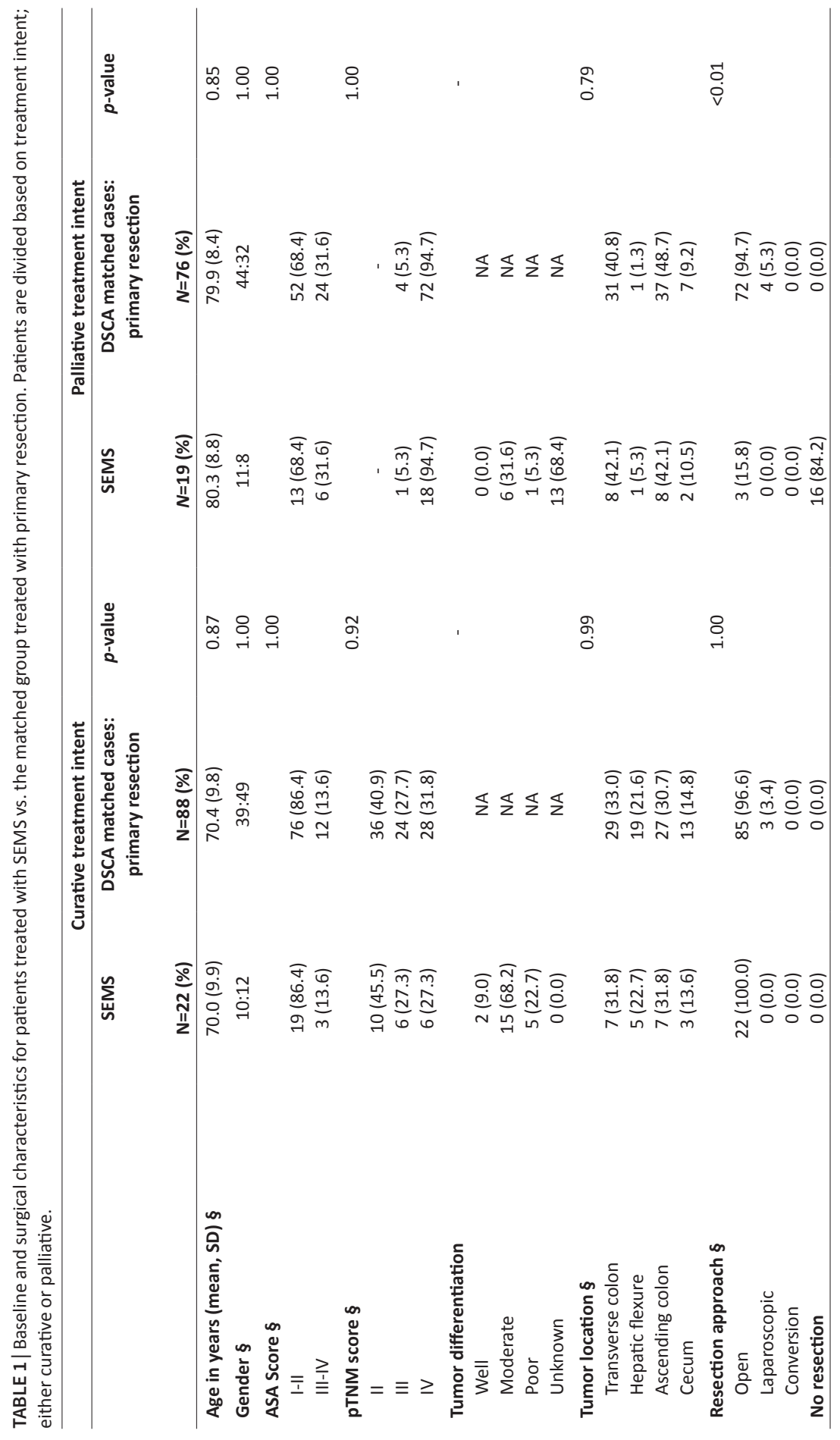




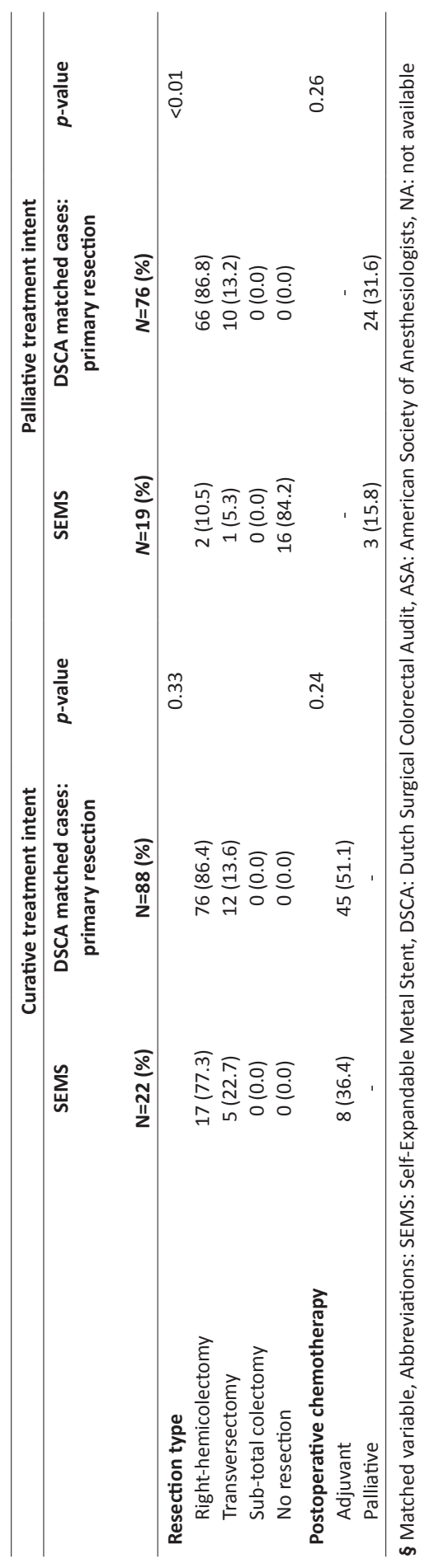


TABLE 2 | SEMS-related characteristics and outcomes

\begin{tabular}{|c|c|c|c|}
\hline & $\begin{array}{l}\text { SEMS total } \\
\mathrm{N}=41(\%)\end{array}$ & $\begin{array}{l}\text { SEMS as BTS } \\
\qquad \begin{array}{l}\mathrm{N}=\mathbf{2 2}(\%) \\
\end{array}\end{array}$ & $\begin{array}{l}\text { SEMS palliative } \\
\qquad \mathrm{N}=19(\%)\end{array}$ \\
\hline \multicolumn{4}{|l|}{ Stent type } \\
\hline Wallflex & $19(46.4)$ & $10(45.5)$ & $9(47.4)$ \\
\hline Wallstent & $17(41.5)$ & $10(45.5)$ & $7(36.8)$ \\
\hline Evolution & $5(12.2)$ & $2(9.0)$ & $3(15.8)$ \\
\hline Days until resection (SD) & - & $17.5(11.8)$ & - \\
\hline Technical success & $38(92.7)$ & $20(90.9)$ & $18(94.7)$ \\
\hline \multicolumn{4}{|l|}{ Reasons technical failure } \\
\hline $\begin{array}{l}\text { Guidewire not able to pass over the } \\
\text { obstruction }\end{array}$ & $2(4.9)$ & $1(4.5)$ & $1(5.3)$ \\
\hline $\begin{array}{l}\text { Double tumor not suitable for SEMS } \\
\text { placement }\end{array}$ & $1(2.4)$ & $1(4.5)$ & - \\
\hline Clinical success & $37(90.2)$ & $19(86.4)$ & $18(94.7)$ \\
\hline Long-term success§ & - & - & $13(68.4)$ \\
\hline \multicolumn{4}{|l|}{ SEMS related complications } \\
\hline Perforation & $1(2.4)$ & $0(0)$ & $1(5.3)$ \\
\hline Migration & $3(7.3)$ & $0(0)$ & $3(15.8)$ \\
\hline Re-obstruction & $5(12.2)$ & $0(0)$ & $5(26.3)$ \\
\hline
\end{tabular}

$\S$ Long-term success is defined as successful relief of obstructive symptoms until death without the need for additional re-interventions. Abbreviations: SEMS: Self-Expandable Metal Stent, BTS: Bridge to Surgery.

\section{DISCUSSION}

The results of this comparative study indicate no difference in morbidity or mortality when SEMS placement and emergency resection as treatment options are compared. However, significantly fewer patients were left with a temporary stoma in the SEMS group. Furthermore, our results indicate that stent placement in the proximal colon might not be more technically demanding than in the distal colon.

A strength of this study is its case-control design. This design enables control for all known confounding factors such as age and ASA-score, hereby ensuring comparability of treatment groups and minimalizing possible confounders. Furthermore, inclusion of all consecutive SEMS patients makes selection bias highly unlikely. In addition, controls were selected from a nationwide database containing over 50.000 patients with colorectal cancer. This led to the possibility of selecting four controls per case, enhancing the power of the current study.

Unfortunately, several limitations of the study should also be kept in mind. First, as this is a retrospective study, data quality may be less optimal than in prospective series. However, all SEMS cases as well as the DSCA database ware recorded prospectively. Second, only short-term outcomes are collected in the DSCA database and since all data are registered anonymously, 


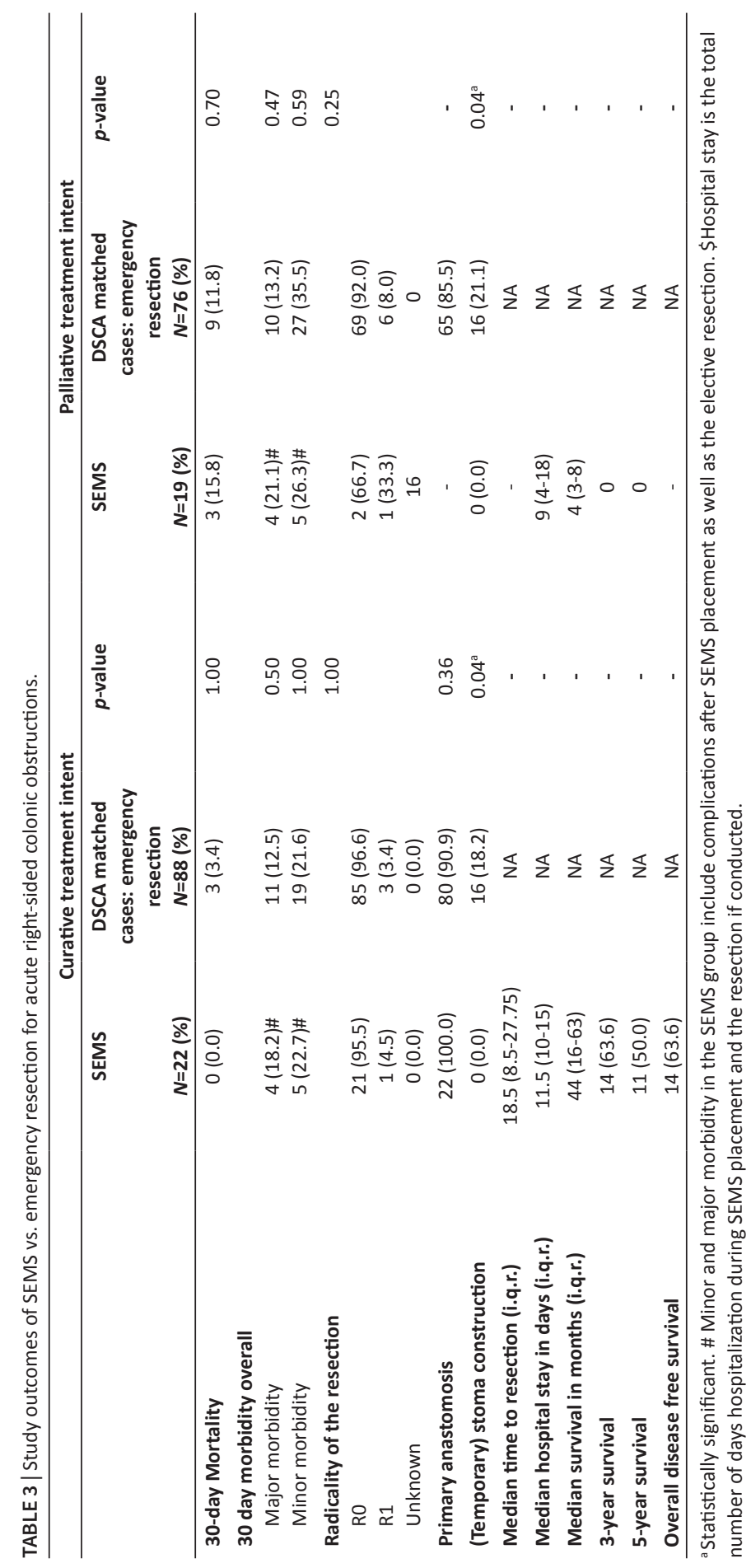


FIGURE 2 | Overall survival of patients treated with a SEMS for a MOPC. Overall survival was stratified according to treatment intent: palliative or curative. Abbreviations: OS: overall survival, DFS: disease free survival

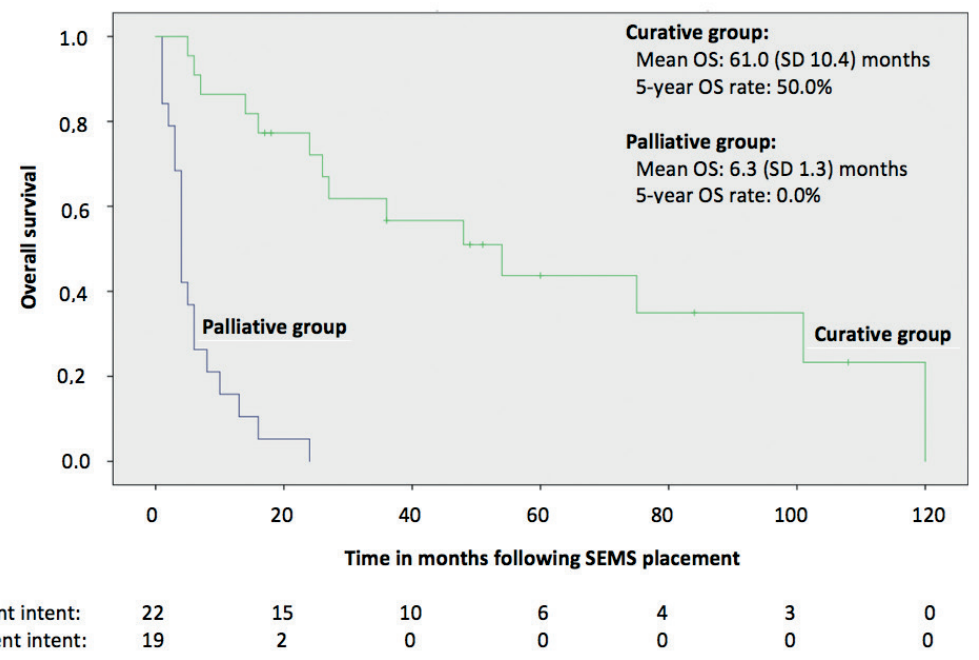

No. at risk

Curative treatment intent:

Palliative treatment intent:

Palliative group:

Mean OS: 6.3 (SD 1.3) months

vear OS rate: $0.0 \%$

it was impossible to retrieve long-term outcomes in the control group. Third, the number of patients was limited. However, even though this is not the largest reported series on proximal stent placement, it is, to our knowledge, the first comparative study available. Since our study design is far less prone to (selection) bias than the previously reported cohort studies on SEMS placement, we do not consider the limited number of patients as a major limitation.

In 2015 a systematic review comparing SEMS as BTS with emergency resection as treatment options for MOPC was published. This study concluded that stent placement led to lower mortality and morbidity rates, fewer colostomies and fewer anastomotic leakages. ${ }^{8}$ However, this systematic review was based on older cohort studies and included a limited number of patients. Despite these limitations, their results were underlined by a recent population-based analysis that compared SEMS as BTS with emergency resection for MOPC. ${ }^{9}$ However, since this was a retrospective analysis, selection bias might have greatly influenced their results, especially since it was unknown why some patients underwent SEMS placement and some underwent emergency resection. ${ }^{9}$ The current study could not confirm the significant difference in morbidity and mortality rates as found in the previous studies. This discrepancy could be explained by the fact that selection bias was minimalized in the current study due to its casecontrol design. On the other hand, the significantly lower temporary stoma rates following SEMS placement found in previous studies could be underlined in the present study. $8,9,20$

Contrary to proximal obstructions, SEMS placement has been extensively evaluated for distal obstructions. A recently conducted Dutch population-based analysis showed mortality rates in patients with severe co-morbidity ( $A S A \geq 3$ ) and/or high age ( $\geq 70$ years) to be $10.3 \%$ following 
TABLE 4 | Comparison of SEMS insertion between left- and right-sided colonic obstruction.

\begin{tabular}{|c|c|c|c|}
\hline Baseline & $\begin{array}{l}\text { Left-sided } \\
N=97(\%)\end{array}$ & $\begin{array}{l}\text { Right-sided } \\
N=41 \text { (\%) }\end{array}$ & $p$-value \\
\hline Age in years (SD) & $73.2(13.0)$ & $74.7(10.7)$ & 0.51 \\
\hline Male gender & $54(55.7)$ & $21(51.2)$ & 0.63 \\
\hline ASA score & & & 0.09 \\
\hline $\mathrm{I}-\mathrm{II}$ & $81(83.5)$ & $32(78)$ & \\
\hline III-IV & $16(16.5)$ & $9(22)$ & \\
\hline Tumor stage & & & 0.68 \\
\hline pTNM I-II & $28(29.8)$ & $10(24.4)$ & \\
\hline pTNM III-IV & $66(70.2)$ & $21(75.6)$ & \\
\hline Treatment intent & & & 0.92 \\
\hline Palliative & $44(45.4)$ & $19(46.3)$ & \\
\hline Curative & $53(54.6)$ & $22(53.7)$ & \\
\hline Stent type & & & 0.12 \\
\hline Wallflex & $27(28.1)$ & $7(17.1)$ & \\
\hline Wallstent & $35(45.8)$ & $17(41.5)$ & \\
\hline Ultraflex & $1(1.0)$ & $0(0.0)$ & \\
\hline Evolution & $13(13.5)$ & $5(12.2)$ & \\
\hline Unkown & $11(11.5)$ & $12(29.2)$ & \\
\hline Technical success & 93 (95.9) & $38(92.7)$ & 0.43 \\
\hline Reasons technical failure & & & 1.00 \\
\hline Guidewire not able to pass over the obstruction & 4 & 2 & \\
\hline Double tumor not suitable for SEMS placement & 0 & 1 & \\
\hline Clinical success & $80(82.3)$ & $37(90.5)$ & 0.31 \\
\hline 30-day morbidity\# & $18(18.8)$ & $10(24.4)$ & 0.49 \\
\hline 30-day mortality\# & $2(2.1)$ & $0(0)$ & \\
\hline Silent perforation & 0 & 0 & 1.00 \\
\hline Median hospital stay in days (i.q.r.) & $12(6-15)$ & $11(7.5-16.5)$ & 0.66 \\
\hline Median time to resection in days (i.q.r.) & $16.5(10-25.5)$ & $18.5(8.5-27.75)$ & 0.54 \\
\hline Long-term success (palliative) & $33 / 41(80.5)$ & $13 / 19(68.4)$ & 0.33 \\
\hline
\end{tabular}

Long-term success is defined as palliative treatment with SEMS without the need for additional surgical interventions. \#30-day morbidity and mortality include only events in the 30-days following SEMS placement; any events following a possible elective resection are not included.

emergency resection, compared to $7.2 \%$ in the SEMS group $(p<0.05)$. These results led to the current recommendation in the Dutch Guideline on Colorectal Surgery to refrain from emergency resection in patients with an ASA-score $\geq 3$ and/or aged $\geq 70$ years, but instead perform decompression as BTS. ${ }^{21}$ Our data indicate that these recommendations might also be beneficial for patients with MOPC. This study also found a $3 \%$ decrease in mortality in the SEMS group. However, our numbers might have been too small to obtain statistical significance.

It is important to emphasize that colonic stent placement should be performed by an experienced endoscopist. For distal stenting, the Dutch Guideline demands experience of at least 20 colorectal stent procedures. Proximal stenting might be even more technically demanding due to 1) a longer distance from anus to obstruction site and an increased colon tortuosity, ${ }^{22-24} 2$ ) the inability to prepare the colon since oral preparation cannot be given and 
enemas only clean distal bowel and 3) the fact that proximal malignant obstructions tend to be more severe since fecal contents are fluid at this point and clinical and radiological ileus requires an absolute obstruction. All these factors require a skilled endoscopist to successfully perform the procedure. Indeed, low technical success rates for proximal stenting have been reported in the past. ${ }^{22,24}$ The present study, however, demonstrates similar success rates for proximal and distal stenting, which is in accordance with other recent studies. ${ }^{25,26}$ It has been suggested that the high technical and clinical success rates found in retrospective studies, might be the result of selection bias for "easy-to-stent cases". ${ }^{27}$ However, in our hospital all consecutive patients with a malignant colonic obstruction were treated with SEMS, making selection bias highly unlikely. This study shows that in experienced hands, SEMS placement in the proximal colon is safe and might be less technically demanding than previously thought.

An ongoing discussion regarding stent-placement in curative patients concerns its influence on long-term oncological outcomes. The Stent-In II group showed six stent-related tumor perforations, eventually leading to local tumor seeding in three patients. ${ }^{28}$ In addition, the Stent-In I study showed development of silent micro-perforations at the site of stent ends in some patients. ${ }^{29}$ The oncological consequences of these adverse events may potentially be dangerous although firm evidence is lacking ${ }^{19,30,31}$ and not clearly demonstrated in other studies..$^{20,32,33}$ In addition, in a previous study conducted at our institution, no differences in recurrence rates, disease free and overall survival were found when SEMS and emergency resection were compared as treatment options for LSCO. ${ }^{34,35}$

In palliative patients the oncological outcomes are far less relevant and the current study shows that SEMS placement could provide a minimally invasive and fast treatment approach. This is in concordance with the European guideline on SEMS-placement for LSCO, stating that SEMS-placement is the preferred palliative treatment approach, except when eGF-blocking agents are used. ${ }^{21}$ Nonetheless, one in three palliative patients in the present study developed long-term complications. These long-term SEMS-related complication rates are similar to previously reported studies. ${ }^{7}$ Fortunately, SEMS-related perforation rate was low, although one end-stage cancer patient died as a consequence (2.4\%). All other complications could be treated, either by re-stenting or by surgery. Overall, SEMS placement provided successful relief of obstructive symptoms until death in approximately $70 \%$ of patients, sparing them from major abdominal surgery.

SEMS placement for MOPC appears to be a feasible and relatively safe alternative for emergency resection in both the curative and palliative setting. Our data indicate that the current recommendation for application of left-sided colonic stenting seem also applicable to proximal obstructions, with the same precautions about age and frail patient groups, and importance of endoscopist experience to minimize risk of perforation and tumor spill. 


\section{REFERENCES}

1. Setti Carraro PG, Segala M, Casana B, Tiberio G. Obstructing colonic cancer: failure and survival patterns over a ten-year follow up after one-stage curative surgery. Dis Colon Rectum 2001;44;243-50

2. Jullumstro E, Wibe A, Lydersen S, Edna TH. Colon cancer incidence, presentation, treatment and outcomes over 25 years. Colorectal Dis 2011;13:512-8

3. Cheynel N, Cortet M, Lepage C, Benoit L, Fauvre J, Bouviar AM. Trends in frequency and management of obstructing colorectal cancers in a well-defined population. Dis Colon Rectum 2007;50:1568-75

4. Tanis PJ, Paulino Pereira NR, van Hooft JE, Consten ECJ, Bemelman WA. Resection of Obstructive Left-Sided Colon Cancer at a National Level: A Prospective Analysis of Short-Term Outcomes in 1,816 Patients. Dig Surg 2015;4;32(5):317-324

5. Huang X, Lv B, Zhang S, Meng L. Preoperative colonic stents versus emergency surgery for acute left-sided malignant colonic obstruction: a meta-analysis. J Gastrointest Surg 2014;18(3):584-91

6. Amelung FJ, Mulder CLJ, Verheijen PM, Draaisma WA, Consten ECJ. Acute resection versus bridge to surgery with diverting colostomy for patients with acute malignant left-sided colonic obstruction: systematic review and meta-analysis. Surgical Oncology 2015;24:313-321

7. Zhao XD, Cai BB, Cao RS, Shi RH. Palliative treatment for incurable malignant colorectal obstructions: a metaanalysis. World J Gastroenterol 2013;7;19(33):5565-74

8. Amelung FJ, de Beaufort HWL, Siersema PD, Verheijen $\mathrm{PM}$, Consten ECJ. Emergency resection versus bridge to surgery with stenting in patients with acute right-sided colonic obstruction: a systematic review focusing on mortality and morbidity rates. Int J Colorectal Dis 2015;30:1147-1155

9. Amelung F.J. Consten E.C.J., Siersema PD, Tanis PJ. A population-based analysis of three treatment modalities for malignant obstruction of the proximal colon: acute resection versus stent or stoma as a bridge to surgery. Ann Surg Oncol 2016;23(11):3660-8

10. McArdle CS, Hole DJ. Emergency presentation of colorectal cancer is associated with poor 5-year survival. Br J Surg 2004;91:605-609

11. Sjo OH, Larsen S, Lunde OC, Nesbakken A. Short term outcome after emergency and elective surgery for colon cancer. Color Dis 2009;11:733-739
12. Smithers BM, Theile DE, Cohen JR, Evans EB, Davis NC. Emergency right hemicolectomy in colon carcinoma: a prospective study. Aust N Z J Surg 1986;56:749-752

13. Kobayashi H, Miyata H, Gotoh M, Baba H, Kimura W, Kitagawa Y, Nakagoe T, Shimada M, Tomita N, Sugihara $\mathrm{K}$, Mori M. Risk model for right hemicolectomy based on 19, 070 Japanese patients in the National Clinical Database. J Gastroenterol 2013;49;1047-55

14. Smothers L, Hynan L, Fleming J, Turnage R, Simmang C, Anthony T. Emergency surgery for colon carcinoma. Dis Colon Rectum 2003;46:24-30

15. Fletcher RW, Fletcher SW, Fletcher GS. Clinical Epidemiology - the essentials. Chapter 11. $5^{\text {th }}$ Edition, Lipincott Williams \& Wilkins

16. Van Leersum NJ, Snijders HS, Henneman D, kolfschoten NE, Gooiker GA, ten Berge MG, Eddes EH, Wouters MW, Tollenaar RA, Bemelman WA, van Dam RM, Elferink MA, Karsten TM, van Krieken JH, Lemmens VE, Rutten HJ, Menasama ER, van de Velde CJ, Meijerink WJ, Wiggers T, van der Harst E, Dekker JW, Boerma D. The Dutch surgical colorectal audit. Eur J Surg Oncol 2003;39:10631070

17. Dindo D, Demartines N, Clavien PA. Classification of surgical complications: a new proposal with evaluation in a cohort of 6336 patients and results of a survey. Ann Surg 2004;240: 205-213

18. Koch M, Kienle P, Sauer P, Willeke F, Buhi K, Benner A, Lehnert T, Herfahrt C, von Knebel Doeberitz M, Weitz J. Hematogenous tumor cell dissemination during colonoscopy for colorectal cancer. Surg Endosc 2004;18:587-59

19. Maruthachalam K, Lash GE, Shenton BK, Horgan AF. Tumour cell dissemination following endoscopic stent insertion. Br J Surg 2007;94:1151-1154

20. Kye B H, Lee YS, Cho HM, Kim JG, Oh ST, Lee IK, Kang WK, Ahn CH, Lee SC, Park JK, Kim HJ. Comparison of long-term outcomes between emergency surgery and bridge to surgery for obstruction in right-sided colon cancer: a multicenter retrospective study. Ann Surg Oncol 2016;23;1867-74

21. van Hooft JE, van Halsema EE, Vanbiervliet $G$, Beets-Tan RG, De Vitt JM, Donnellan F, Dumoncaeu JM, GlynneJones RG, Hassan C, Jimenez-Perez J, Meisner S, Muthusamy VR, Parker MC, Rigembeau JM, Sabbagh C, Sagar J, Tanis PJ, Vandervoort J, Webster GJ, Manes G, Barthet MA, Repici A. Self expandable metal stents for obstructing colonic and extracolonic cancer: European Society of Gastrointestinal Endoscopy (ESGE) Clinical Guideline. Gastrointest Endosc 2014;80:747-761 
22. Cho YK, Kim SW, Lee BI, Lee KM, Lim CH, Kim JS, Chag JH, Park JM, Lee IS, Choi MG, CHoi KY, Chung IS. Clinical outcome of self-expandable metallic stent placement in the management of proximal colon obstruction. Gut liver 2011;5: 165-170

23. Y. Saida, J. Nagao, Y. Nakamura. Trans-anal decompression for colorectal obstruction. Gastroent Endosc 2008;50;80-90

24. Bonin EA, Baron TH. Update on the Indications and Use of Colonic Stents. Curr Gastroenterol Rep 2010;12:37482

25. Moroi R, Endo K, Ichikawa R, Nagai H, Shinkai H, Kimura $T$, Ishiyama F, Yaguchi K, Kayaba S, Shimosegawa T. The Effectiveness of Self-Expandable Metallic Stent Insertion in Treating Right-Sided Colonic Obstruction: A Comparison between SEMS and Decompression Tube Placement and an Investigation of the Safety and Difficulties of SEMS Insertion in Right Colons. Gastroenterol Res Pract. 2014:372918

26. Tal AO, Friedrich-Rust M, Bechstein WO, Woeste G, Trojan J, Zeuzeum S, Sarrazin C, Albert JG. Selfexpandable metal stent for malignant colonic obstruction: outcome in proximal vs. left sided tumor localization. J Gastroenterol 2013;51:551-7

27. Ansaloni L, Andersson RE, Bazzoli F, Catena F, Cennamo V, Di Saverio S, Fuccio L, Jekkel H, Leppaniemi A, Moore E, Pinna AD, Pisano M, Repici A, Sugarbaker PH, Tuech JJ. Guidelines in the management of obstructing cancer of the left colon: consensus conference of the world society of emergency surgery (WSES) and peritoneum and surgery (PnS) society. World I Emerg Surg 2010;28(5):29

28. Sloothaak DA, van den Berg MW, Dijkgraaf MG, Fockens P, Tanis PJ, van Hooft JE, Bemelman WA. Collaborative Dutch Stent-In study group.Oncological outcome of malignant colonic obstruction in the Dutch Stent-In 2 trial. Br J Surg 2014;101(13):1751-7
29. van Hooft JE, Fockens P, Marinelli AW, Bossuyt PM, Bemelman WA. Premature closure of the Dutch Stentin I study. Lancet 2006;368:1573-1574

30. Erichsen R, Horváth-Puhó E, Jacobsen JB, Nilsson T, Baron JA, Sorensen HT. Long-term mortality and recurrence after colorectal cancer surgery with preoperative stenting: a Danish nationwide cohort study. Endoscopy 2015;47:517-24

31. Sabbagh C, Browet F, Diouf M, Cosse C, Brehant O, Bartolli E, Mauvais F, Chauffert B, Dupas JL, NguyenKhac E, Regimbeau JM. Is Stenting as "a Bridge to Surgery" an Oncologically Safe Strategy for the Management of Acute, Left-Sided, Malignant, Colonic Obstruction?: A Comparative Study With a Propensity Score Analysis. Ann Surg 2013;258:107-115

32. Tung KL, Cheung HY, Ng LW, Chung CC, Li MK. Endolaparoscopic approach versus conventional open surgery in the treatment of obstructing left-sided colon cancer: a long-term follow up of a randomized trial. Asian J Endosc Surg 2013;6:78-81

33. Choi JM, Lee $\mathrm{C}$, Han YM, Lee $\mathrm{M}$, Choi YH, Jang DK, Im JP, Kim SG, Kim JS, Jung HC. Long-term oncologic outcomes of endoscopic stenting as a bridge to surgery for malignant colonic obstruction: comparison with emergency surgery. Surg Endosc. 2014;28(9):2649-55

34. Van den Berg MW, Sloothaak DAM, Dijkgraaf MGW, van der Zaag ES, Bemelman WA, Tanis PJ, Bosker RJ, Fockens $P$, ter Borg F, van Hooft JE. Bridge to surgery stent placement versus emergency resection for acute malignant colonic obstruction. Br J Surg 2014;101 (7):867-73

35. Integraal Kankercentrum Nederland. National Working Group on Gastrointestinal Cancers. Guideline Colon Cancer 2.0. Available at: http://www.oncoline.nl/ coloncarcinoom. Accessed January 5th, 2016

36. Zhao XD, Cai BB, Cao RS, Shi RH. Palliative treatment for incurable malignant colorectal obstructions: a metaanalysis. World J Gastroenterol 2013;7;19(33):5565-74 


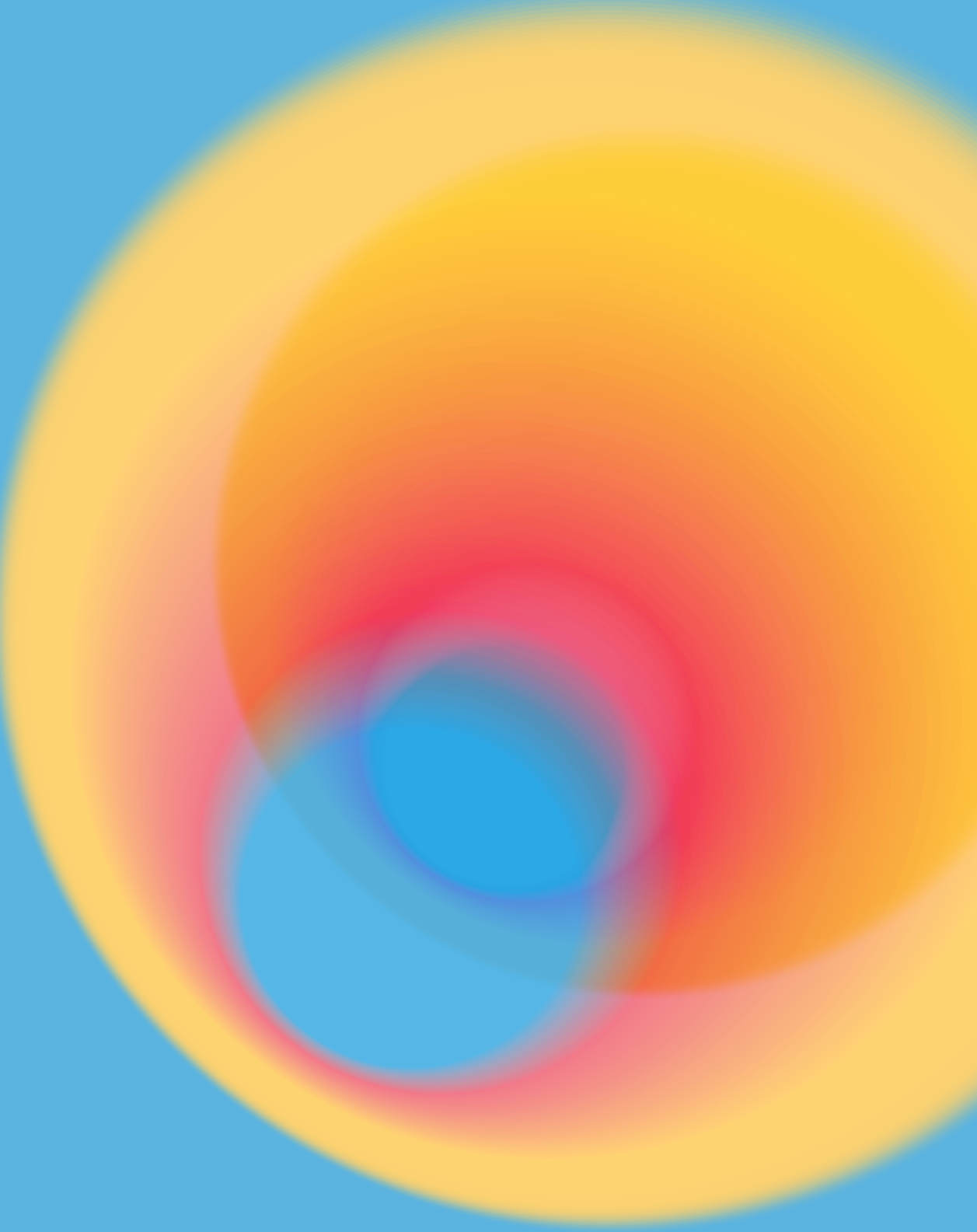




\section{PART II LEFT-SIDED COLONIC OBSTRUCTIONS}





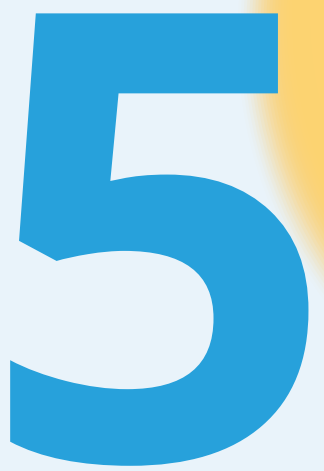

Acute resection versus bridge to surgery with diverting colostomy for patients with acute malignant left sided colonic obstruction: systematic review and meta-analysis

Surgical Oncology 2015
F.J. Amelung
C.L.J. Mulder
P.M. Verheijen
W.A. Draaisma
P.D. Siersema
E.C.J. Consten 


\section{ABSTRACT}

\section{Background}

Currently, no consensus exists on the best treatment strategy for acute malignant left-sided colonic obstruction. This systematic review and meta-analysis aims to compare the outcomes following the two surgical treatment options; primary resection versus colostomy creation as bridge to surgery.

\section{Methods}

This systematic review was performed using the Preferred Reporting Items for Systematic Reviews and Meta-Analyses (PRISMA) guidelines to minimize risk of bias. Pubmed, Embase and Cochrane Library were searched for all relevant literature. Methodological quality of included studies was assessed using the MINORS criteria. Pooled odds ratios with $95 \%$ confidence intervals $(95 \% \mathrm{Cl})$ were calculated using random effects models.

\section{Results}

Eight comparative studies were included, reporting on 2424 patients; 1973 patients were treated with primary resection and 451 patients with colostomy construction followed by elective resection. Meta-analysis showed no significant differences between both treatment groups regarding 30-day mortality and morbidity $(\mathrm{OR}=0.77,95 \% \mathrm{Cl} 0.3-1.96$ and $\mathrm{OR}=0.76$, $95 \% \mathrm{Cl} 0.51-1.13$, respectively). However, patients treated with a colostomy followed by elective resection had significantly more primary anastomoses constructed and were less likely to be left with a permanent colostomy $(\mathrm{OR}=0.17,95 \% \mathrm{Cl} 0.11-0.26$ and $\mathrm{OR}=0.22,95 \% \mathrm{Cl} 0.11-0.46$, respectively).

\section{Conclusion}

This systematic review provides an overview of all available literature on primary resection versus colostomy creation as bridge to surgery in patients with acute LSCO. Keeping the limitations of this study in mind, we conclude that a diverting colostomy as bridge to surgery is a safe and valid alternative for primary resection. 


\section{INTRODUCTION}

As much as $8-13 \%$ of all patients with colorectal cancer present with an acute left-sided colonic obstruction (LSCO). ${ }^{1,2}$ The majority of these patients are elderly and in a poor general condition due to several days of reduced intake, weight loss and severe bowel distension. ${ }^{3,4}$ In addition, patients presenting with an acute bowel obstruction are known to generally have a more advanced tumor stage compared to electively treated patients. ${ }^{5,6}$ All these factors contribute to an increased operative risk, resulting in high postoperative mortality and morbidity rates. However, when total obstruction is present, immediate intervention is required to prevent life-threatening complications due to extensive bowel dilation.

At present, three different treatment modalities for patients with LSCO exist; 1) primary resection, 2) stent placement as bridge to surgery or 3 ) diverting colostomy construction as a bridge to surgery. Both stent placement as well as colostomy construction address the immediate problem of a colonic obstruction and yet create time for optimization of the patients' condition prior to elective resection. Furthermore, accurate tumor staging can be achieved and neoadjuvant treatment can be considered as a possible interference if indicated. ${ }^{7,8}$

Stenting as bridge to surgery has been proposed as an attractive alternative to primary resection. Several meta-analyses have reported on favorable short-term outcomes of stent placement when compared with primary resection. ${ }^{9,10}$ However, stent placement for LSCO has recently been debated since several randomized trials were closed prematurely due to the high rate of stent-related complications. ${ }^{11-13}$ Furthermore, questions have been raised about the oncological long-term consequences of stent placement. ${ }^{14}$

This has led to a search for a valid treatment alternative for patients with an acute LSCO that is associated with fewer procedure-related complications. Successful colonic decompression in patients with acute LSCO is almost guaranteed when a diverting colostomy is used; hereby addressing the immediate problem and additionally providing the aforementioned benefits of a bridge to surgery approach. Furthermore, since the tumor is not manipulated directly such as is the case with stent placement, no influence on long-term oncologic outcomes is expected. Even though literature on stent placement for LSCO is extensive, studies reporting on diverting colostomy as bridge to surgery are limited.

The current study aims to provide an overview of procedure related mortality and morbidity rates of both primary resection and a diverting colostomy as bridge to surgery in patients with acute LSCO through a systematic review of the literature and subsequent meta-analysis.

\section{METHODS}

This systematic review was performed using the Preferred Reporting Items for Systematic Reviews and Meta-Analyses (PRISMA) guidelines to minimize risk of bias. ${ }^{15}$ 


\section{Search strategy}

A systematic literature search was conducted in EMBASE (Ovid), MEDLINE (Pubmed) and the Cochrane Database of Systematic Reviews. There were no restrictions to the search. The final search was conducted on August 24, 2015. The search terms were synonyms for acute left sided colon obstruction, primary resection and diverting colostomy as bridge to surgery. Search terms used in EMBASE were: (('acute obstruction':ab,ti OR ileus:ab,ti OR 'colon obstruction':ab,ti OR 'colonic obstruction':ab,ti OR 'intestinal obstruction':ab,ti OR 'malignant obstruction':ab,ti OR 'rectosigmoidal obstruction': ab,ti OR 'left-sided obstruction':ab,ti OR 'distal obstruction':ab,ti OR 'large bowel obstruction':ab,ti) AND (surgery:ab,ti OR colectomy:ab,ti OR resection:ab,ti OR 'three stage procedure':ab,ti OR 'bridge to surgery':ab,ti OR 'staged resection':ab,ti OR ostomy:ab,ti OR Hartman*:ab,ti OR stoma:ab,ti). MEDLINE and Cochrane Database were searched using similar search terms. Articles deemed potentially relevant were screened full text for inclusion. In addition, potentially important references of all included studies were also assessed.

\section{Inclusion and exclusion criteria}

All literature on primary resection and/or colostomy as bridge to surgery for acute malignant LSCO was considered for inclusion. Studies were included when they met the following criteria: 1) the obstruction was caused by primary colon carcinoma in at least $80 \%$ of patients, 2) patients included had a left-sided obstruction; defined as a tumor location distal of the splenic flexure and proximal of the rectum 3 ) at least $70 \%$ of the patients receiving a colostomy was treated with curative intent and underwent elective resection and 4) data on 30-day mortality and/or morbidity was provided. Exclusion criteria were studies in languages other than English or Dutch, non-comparative studies, studies that included fewer than 10 patients and studies performed on animals.

\section{Study selection and assessment of methodological quality}

Two reviewers (FA and CM) independently screened all citations for eligibility based on title and abstract. Title and abstract of all articles were verified for the previously stated in- and exclusion criteria. Both reviewers read the full text of the remaining articles after initial screening. Final inclusion was based on consensus. Methodological quality of the studies was assessed using the MINORS criteria. ${ }^{16}$

\section{Outcomes of interest}

Primary outcomes were mortality and morbidity following the different treatment strategies. Mortality was defined as death within 30 days of the intervention. Morbidity was subdivided in major and minor morbidity. Major morbidity was defined as morbidity grade IIIb or higher and minor morbidity as IIla or lower, as defined by the classification of surgical complications developed and validated by Clavien and Dindo et al. ${ }^{17}$ Secondary outcomes were percentage of patients with anastomotic leakage, permanent colostomy and total length of hospital stay following both treatment strategies. 


\section{Data extraction}

Baseline characteristics, procedure related mortality, morbidity, the number of completed anastomoses after resection, the number of patients left with a permanent colostomy and mean hospital stay were collected from all included studies.

\section{Statistical analysis}

Data analyses were performed using Review Manager 5.3 software (the Cochrane Collaboration 2012, the Nordic Cochrane Centre, Copenhagen, Denmark). Heterogeneity among trials was assessed using the Cochrane $Q$ statistic $(P<0.10$ was considered representative of statistically significant heterogeneity) and the $\mathrm{I}^{2}$ statistic $\left(\mathrm{I}^{2}>50 \%\right.$ was considered to represent significant heterogeneity). ${ }^{18} \mathrm{~A}$ random-effects model was used for analysis given the variability of methods and populations in the included studies. Results are presented as odds ratios (ORs) with $95 \%$ confidence intervals $(95 \% \mathrm{Cls})$. When significant heterogeneity was found, we tried to identify factors that might explain the heterogeneity. If possible a sub-analysis was conducted correcting for these factors. When no determinant for identified heterogeneity could be found, no metaanalysis was conducted for that outcome measure; instead, the outcomes per included study were reported separately. For continuous variables, the weighted mean difference was calculated with values below 1 favoring colostomy. To identify possible publication bias, a funnel plot of all primary outcomes was conducted.

\section{RESULTS}

\section{Study selection}

The initial search yielded 18.175 relevant citations, 11.986 remained after removal of all duplicates (Figure 1). Initial screening excluded 11.955 articles based on title and abstract. The full text of the remaining 31 articles was reviewed. After detailed analysis, another 25 articles were excluded. Reasons for exclusion were a non-compatible patient group $(n=4)$ or intervention ( $n=5)$, non-comparative study design $(n=3)$, no report on outcomes for left-sided obstructions specifically $(n=7)$, language other than English/Dutch $(n=3)$, review article $(n=2)$ and presentation of interim results $(n=1)$. Two additional studies were included after crossreference.

\section{Study characteristics}

The included studies were published between 1977 and 2015 and report outcomes on a total of 2424 patients; 1735 patients in the primary resection group and 451 patients in the colostomy group. Baseline characteristics are shown in Table 1. Mean age for patients undergoing primary resection ranged from $67.3-71.0$ years and $54.7 \%$ was male. For patients treated with colostomy and elective resection mean age ranged between $68.2-70.0$ years, and $59.7 \%$ was male. Due to lack of reporting in the included studies, ASA classification and tumor stage could not be documented for the separate treatment groups.

All 451 patients included in the colostomy group received a loop colostomy as initial decompression method; in 422 patents (94\%) loop colostomy was constructed as a bridge to 
FIGURE 1 | Flowchart of the search, performed on August 24, 2015

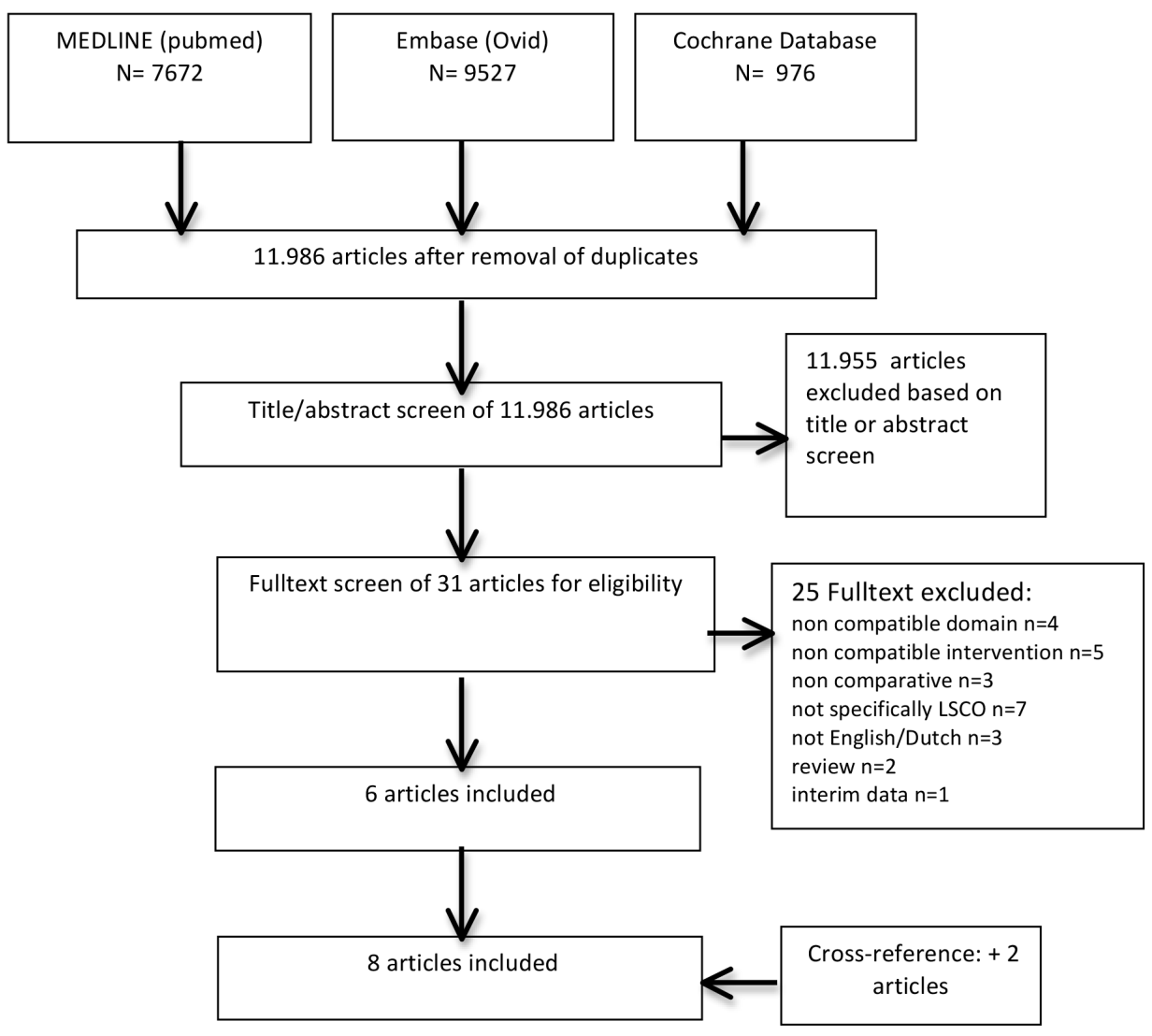

surgery, whereas in the remaining 29 patients it was as a palliative procedure only (Figure 2). The specific type of colostomy was not specified in three out of eight studies and is therefore unknown in 171 patients. ${ }^{19-21}$ Of the remaining 280 patients, 260 received a loop transverse colostomy, 13 a ceacostomy and 7 a loop sigmoid colostomy. ${ }^{7,22-25}$ Unfortunately, none of the studies specified whether colostomies were created using a laparoscopic or open approach. A total of 1735 patients received primary resection, which included primary resection with the construction of a primary anastomosis (with or without a protective proximal stoma) in 955 patients and a Hartmann's procedure in 744 patients. ${ }^{19-25}$ The specific procedure type was not reported for 36 patients. $^{7}$

All included studies had a comparative design; four were retrospective analyses and four had a prospective design, of which 1 was a randomized trial (Kronborg et al.). The mean MINORS criteria score was 17.4 (range 15 to 20) with a maximum possible score of 24 (Table 2). 
TABLE 1 | Baseline characteristics from included studies evaluating primary resection versus diverting colostomy as bridge to surgery for LSCO.

\begin{tabular}{|c|c|c|c|c|c|c|c|c|}
\hline \multirow[t]{2}{*}{ Author, Year } & \multirow[t]{2}{*}{ Study arm } & \multirow[t]{2}{*}{$\mathbf{N}$} & \multirow{2}{*}{$\begin{array}{c}\text { Age* } \\
\text { (years) }\end{array}$} & \multirow{2}{*}{$\begin{array}{l}\text { Male } \\
\text { gender } \\
\%(n)\end{array}$} & \multicolumn{4}{|c|}{ Duke's stage } \\
\hline & & & & & A & B & C & D \\
\hline \multirow[t]{2}{*}{ Carson, $1977^{19}$} & Primary resection & 9 & NA & NA & NA & NA & NA & NA \\
\hline & Colostomy & 28 & NA & NA & NA & NA & NA & NA \\
\hline \multirow[t]{2}{*}{ Fielding, $1979^{20}$} & Primary resection & 36 & 70.2 & NA & NA & NA & NA & NA \\
\hline & Colostomy & 41 & 68.4 & NA & NA & NA & NA & NA \\
\hline \multirow[t]{2}{*}{ Gandrup, $1992^{21}$} & Primary resection & 23 & NA & NA & NA & NA & NA & NA \\
\hline & Colostomy & 53 & NA & NA & NA & NA & NA & NA \\
\hline \multirow[t]{2}{*}{ Huddy, $1992^{22}$} & Primary resection & 30 & NA & NA & NA & NA & NA & NA \\
\hline & Colostomy & 12 & NA & NA & NA & NA & NA & NA \\
\hline \multirow[t]{2}{*}{ Kronborg, $1995^{24} \S$} & Primary resection & 63 & 71 & $44.4(28)$ & $1.6(1)$ & $63.5(40)$ & $25.4(16)$ & $0(0)$ \\
\hline & Colostomy & 58 & 70 & $39.7(23)$ & $0(0)$ & $48.3(28)$ & $19(11)$ & $5.2(3)$ \\
\hline \multirow[t]{2}{*}{ Tan, $1995^{7}$} & Primary resection & 36 & NA & NA & $0(0)$ & $55.6(20)$ & $38.9(14)$ & $5.6(2)$ \\
\hline & Colostomy & 34 & NA & NA & $0(0)$ & $70.0(21)$ & $30.0(9)$ & $0(0)$ \\
\hline \multirow[t]{2}{*}{ Jiang, $2008^{23}$} & Primary resection & 53 & 67.3 & $81.1(43)$ & NA & NA & NA & NA \\
\hline & Colostomy & 90 & 68.2 & $77.8(70)$ & NA & NA & NA & NA \\
\hline \multirow[t]{2}{*}{ Tanis, $2015^{25}$} & Primary resection & 1485 & 70 & $54.2(805)$ & NA & NA & NA & NA \\
\hline & Colostomy & 135 & 68 & $56.3(76)$ & NA & NA & NA & NA \\
\hline
\end{tabular}

$\mathrm{n}=$ number of patients in study arm; NA=not available. *Mean age, $\S$ Randomized controlled trial

\section{Primary outcomes \\ Mortality}

All included studies reported on mortality rate. ${ }^{7,19-25}$ Comparison between primary resection and colostomy creation did not lead to significantly different mortality rates. The OR was 0.79 $(95 \% \mathrm{Cl}=0.38-1.65)$, as assessed using the Mantel-Haenszel method with random effects model. However, some evidence of heterogeneity was found between studies $(P=0.07$, with a corresponding $\mathrm{I}^{2}=46 \%$ ) (Figure $3 \mathrm{~A}$ ).

A likely cause of this heterogeneity is that publication date varied greatly among the included studies, with the eldest studies dating from the 1970's. In view of the advances in surgical techniques and the fact that primary resection with immediate anastomosis was not widely practiced until the late 1990s as a safe alternative to colostomy, a sub-analysis of all included studies published after 1995 was conducted. The four studies taken into account in this subanalysis all used minimally invasive surgical techniques used in current practice., ${ }^{7,23}$ This sub-analysis revealed no statistically significant heterogeneity among studies $\left(p=0.12, I^{2}=49 \%\right)$ 


\begin{tabular}{|c|c|c|c|c|c|c|c|c|c|c|c|c|}
\hline Author, year & $\begin{array}{l}\frac{E}{\sigma} \\
\frac{1}{\pi} \\
\frac{\mathbb{U}}{U}\end{array}$ & 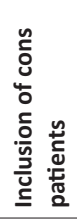 & 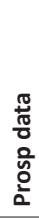 & $\begin{array}{l}\text { 눈 } \\
\text { 융 }\end{array}$ & 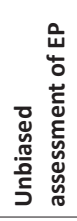 & 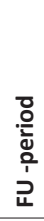 & 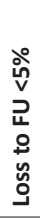 & 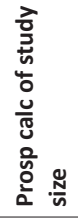 & 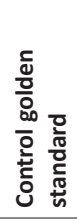 & 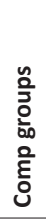 & 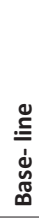 & 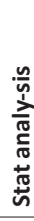 \\
\hline Carson, $1977^{19}$ & 2 & 2 & 1 & 2 & 0 & 1 & 0 & 0 & 2 & 2 & 2 & 2 \\
\hline Fielding, $1979^{20}$ & 2 & 2 & 2 & 2 & 0 & 0 & 0 & 0 & 2 & 2 & 2 & 2 \\
\hline Gandrup, $1992^{21}$ & 2 & 2 & 1 & 2 & 0 & 0 & 0 & 0 & 2 & 2 & 2 & 2 \\
\hline Huddy, $1992^{22}$ & 2 & 2 & 1 & 2 & 0 & 2 & 2 & 0 & 2 & 2 & 2 & 2 \\
\hline Kronborg, $1994^{24}$ & 2 & 2 & 2 & 2 & 1 & 2 & 0 & 0 & 2 & 2 & 2 & 2 \\
\hline Tan, $1995^{7}$ & 2 & 2 & 1 & 2 & 1 & 0 & 0 & 0 & 2 & 2 & 2 & 2 \\
\hline Jiang, $2008^{23}$ & 2 & 2 & 2 & 2 & 0 & 2 & 2 & 0 & 2 & 2 & 2 & 2 \\
\hline Tanis, $2015^{25}$ & 2 & 2 & 2 & 2 & 1 & 1 & 0 & 0 & 2 & 2 & 2 & 2 \\
\hline
\end{tabular}

The MINORs criteria are a validated critical appraisal tool especially suited to grade retrospective comparative studies. A maximum of 2 points per category can be given. When the study does not report on an item to be judged, no points were given. Consec=consecutive, prosp=prospective, app=appropriate, $\mathrm{EP}=$ =ndpoints, $\mathrm{FU}=$ follow up, calc=calculation, comp=comparable, stat=statistical

but also did not result in a significant difference between both treatment groups $(O R=0.77$, 95\% Cl=0.3-1.97) (Figure 3B).

All mortality and morbidity rates in the colostomy group include the initial colostomy construction, as well as the subsequently conducted elective resection. Reasons for mortality were not stated specifically in each study.

\section{Morbidity}

Morbidity was reported in 4 of 8 studies. ${ }^{7,23-25}$ Major morbidity rate, Clavien-Dindo grade IIIb or higher, was $15.6 \%(256 / 1637)$ in the primary resection group versus $12.6 \%(40 / 317)$ in the colostomy group. Analysis showed this not to be significantly different ( $\mathrm{OR}=0.76,95 \% \mathrm{Cl}=0.48$ 1.20). (Figure $3 C$ ). No evidence of statistically significant heterogeneity among studies was observed $\left(p=0.33, l^{2}=13 \%\right)$. Severe heterogeneity was found in studies reporting on minor morbidity with a $\mathrm{P}<0.001$ and a corresponding $\mathrm{l}^{2}=89 \%$. Therefore, data was not pooled for this outcome measure. Minor morbidity rate is reported per individual study in Table 3.

Long-term complications were not taken into account, since all included studies reported on short-term complications only.

\section{Secondary outcomes}

\section{Anastomotic completion and permanent colostomy}

The construction of a primary anastomosis directly following resection was reported in 4 of 8 included studies., ${ }^{73-25}$ In the colostomy group, patients were significantly more likely to have a primary anastomosis constructed following elective resection, when compared to the acute resection group $(\mathrm{OR}=0.17,95 \% \mathrm{Cl}=0.11-0.26)$ (Figure 3D). Although Tan et al. also reported on the number of primary anastomoses constructed following resection this study was not 
FIGURE 2 | Overview of treatment modalities performed in the included patients

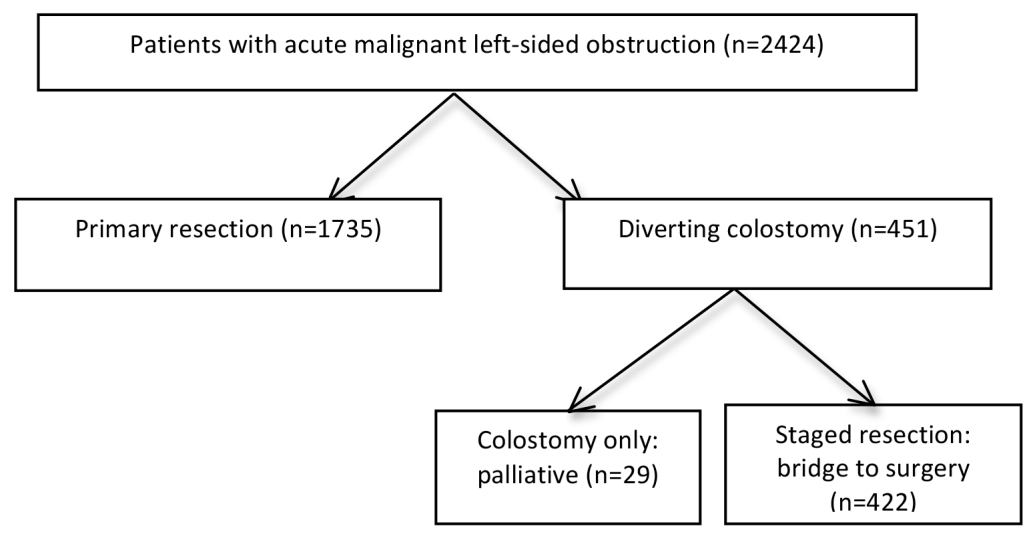

included in meta-analysis, because in this study it was predetermined that all patients treated with primary resection also received a primary anastomosis.

Permanent colostomy rate was $21.6 \%(30 / 139)$ in the primary resection group, compared to $9.4 \%(13 / 201)$ in the colostomy group. Meta-analysis revealed this to be statistically significant $(\mathrm{OR}=0.22,95 \% \mathrm{Cl}=0.11-0.46) .{ }^{21,23,24}$ No evidence of heterogeneity was found $\left(p=0.83, l^{2}=0 \%\right)$ (Figure 3E).

\section{Anastomotic leakage}

Data on anastomotic leakage was only available in 4 included studies, of which only two reported comparative data. Therefore, no meta-analysis was performed. Data on the number of anastomotic leakages per individual study is presented in Table 3., 7,20,22,24

\section{Hospital stay}

Mean hospital stay in days was reported in 6 of 8 studies and was analyzed for both groups. ${ }^{7,21-25}$ Since standard deviation was not reported in most studies, no meta-analysis could be conducted. However, weighed mean difference was 0.92 , meaning that hospital stay was shorter in the colostomy group. The length of hospital stay was defined as the total number of days spent in hospital in order to defunction, resect the tumor and restore bowel continuity in all studies. We also looked at operation time, recurrence and disease free period. However, only two or fewer of the included studies reported on these outcomes, preventing pooling of the data.

\section{Publication bias}

Funnel plots based on the incidence of mortality and morbidity are shown in Figure 4. The shape of both funnel plots did not reveal asymmetry and no study lay outside the limits of the $95 \% \mathrm{Cl}$, indicating no obvious publication bias. 


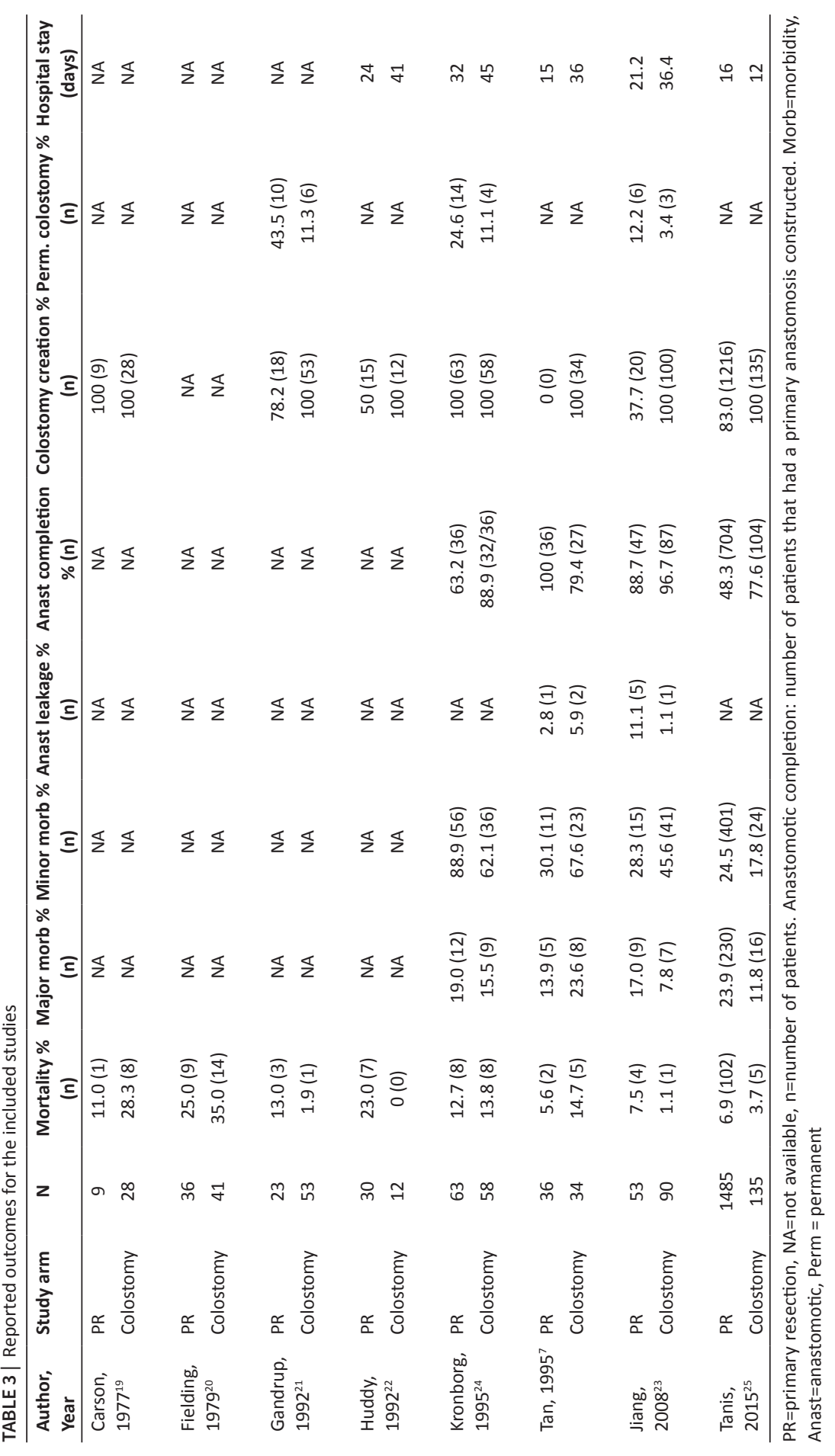


FIGURE 3 | Forest-plots for aggregated data of included studies comparing outcomes after primary resection versus diverting colostomy creation and elective resection in patients with malignant LSCO. A. Mortality B. Sub-analysis on mortality of studies published after 1995. C. Major morbidity. D. Number of patients that did not have a primary anastomosis constructed. E. Number of patients left with a permanent colostomy.

\section{A}

\begin{tabular}{lrrrrr} 
Study or Subgroup & \multicolumn{6}{c}{$\begin{array}{c}\text { Diverting colostomy } \\
\text { Events }\end{array}$} & $\begin{array}{r}\text { Total } \\
\text { Primary resection }\end{array}$ & Events & Total & Weight \\
\hline Carson, 1977 & 8 & 28 & 1 & 9 & $8.1 \%$ \\
Fielding, 1979 & 14 & 41 & 9 & 36 & $19.7 \%$ \\
Huddy, 1992 & 0 & 12 & 7 & 30 & $5.2 \%$ \\
Gandrup, 1992 & 1 & 53 & 3 & 23 & $7.6 \%$ \\
Kronborg, 1995 & 8 & 58 & 8 & 63 & $18.9 \%$ \\
Tan, 1995 & 5 & 34 & 2 & 36 & $11.6 \%$ \\
Jiang, 2008 & 1 & 90 & 4 & 53 & $8.2 \%$ \\
Tanis, 2015 & 5 & 135 & 102 & 1485 & $20.8 \%$ \\
Total (95\% Cl) & & 451 & & 1735 & $100.0 \%$ \\
Total events & 42 & \multicolumn{5}{l}{136} \\
Heterogeneity: Tau $^{2}=0.47 ;$ Chi $^{2}=12.97, \mathrm{df}=7(\mathrm{P}=0.07) ; I^{2}=46 \%$
\end{tabular}

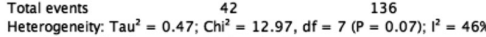

Heterogeneity: $\mathrm{Tau}^{2}=0.47 ; \mathrm{Ch}^{2}=12.97$
Test for overall effect: $\mathrm{Z}=0.63(\mathrm{P}=0.53)$

B

\begin{tabular}{|c|c|c|c|c|c|}
\hline \multirow[b]{2}{*}{ Study or Subgroup } & \multicolumn{2}{|c|}{ Diverting colostomy } & \multicolumn{2}{|c|}{ Primary resection } & \multirow[b]{2}{*}{ Weigh } \\
\hline & Events & Total & Events & Total & \\
\hline Kronborg, 1995 & 8 & 58 & 8 & 63 & $32.0 x$ \\
\hline Tan, 1995 & 5 & 34 & 2 & 36 & $19.2 x$ \\
\hline Jiang, 2008 & 1 & 90 & 4 & 53 & $13.4 x$ \\
\hline Tanis, 2015 & 5 & 135 & 102 & 1476 & $35.4 x$ \\
\hline Total $(95 \% \mathrm{Cl})$ & & 317 & & 1628 & $100.0 \%$ \\
\hline $\begin{array}{l}\text { Total events } \\
\text { Heterogeneity: Tau }{ }^{2} \\
\text { Test for overall effec }\end{array}$ & 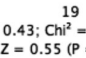 & $d f=$ & $\begin{array}{c}116 \\
(\mathrm{P}=0.12\end{array}$ & & \\
\hline
\end{tabular}

Odds Ratio

Odds Ratio

H, Random, 95\% Cl Year

$3.20[0.34,29.90] 1977$

$1.56(0.58,4.20) 1979$

$0.13[0.01,131] 1992$

$1.10[0.38,3.15] 1995$

$2.93[0.53,16.25] 1995$

$0.14[0.01,1.27] 2008$

$0.52[0.21,1.30] 2015$

$0.79[0.38,1.65]$

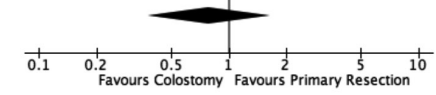

Odds Ratio
H, Random, $95 \% \mathrm{Cl}$ Year

Odds Ratio

Year $\quad M-H_{1}$ Random, $95 \% \mathrm{Cl}$

$1.10[0.38,3.15] 1995$

$2.93[0.53,16.25] 1995$

$0.52[0.21,1.29] 2015$

$0.77[0.30,1.96]$

Test for overall effect: $Z=0.55(P=0.58)$

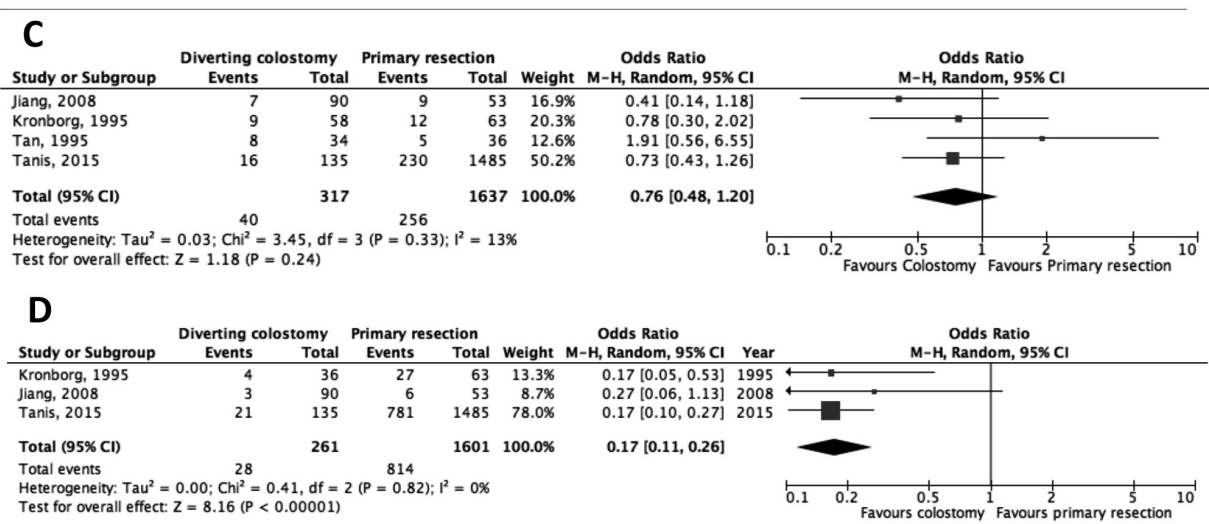

\section{E}

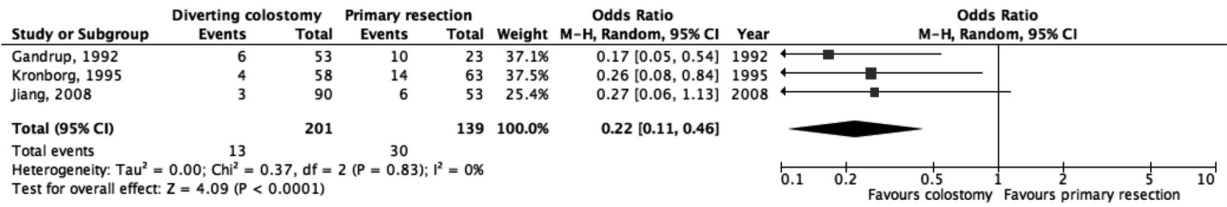


FIGURE 4 | Funnelplots were plotted of all studies that reported on mortality (A) and major morbidity (B) to identify whether publication bias is likely.
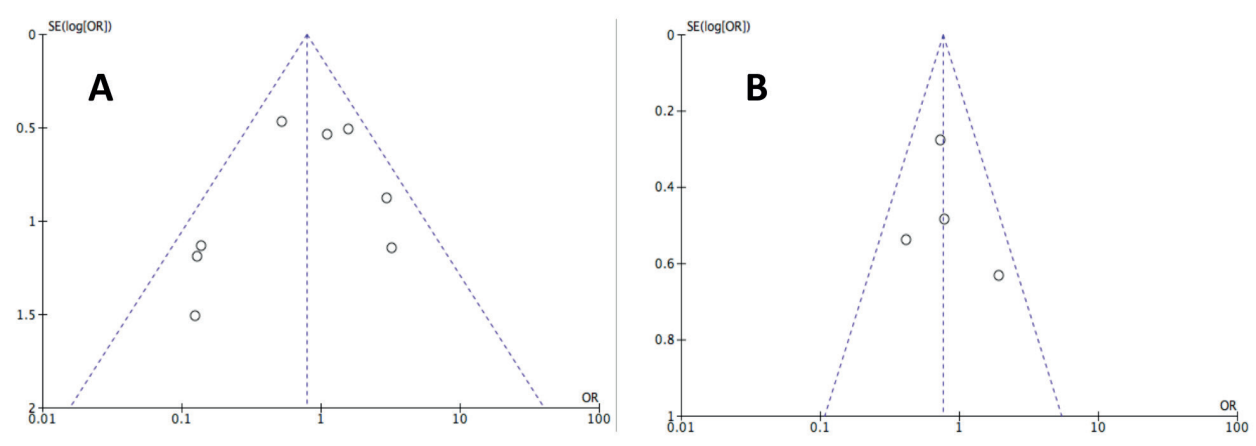

\section{DISCUSSION}

The results of this systematic review on treatment strategies for patients with LSCO suggest that a diverting colostomy as bridge to surgery is a safe and valid alternative to emergency resection. When compared with primary resection, colostomy creation followed by elective resection resulted in significantly more primarily constructed anastomoses $(\mathrm{OR}=0.17$, $95 \% \mathrm{Cl}=0.11-0.26)$ and fewer patients left with a permanent colostomy $(\mathrm{OR} 0.22,95 \% \mathrm{Cl} 0.11$ 0.46). Mortality and major morbidity tended to be lower for the colostomy group, although the difference was not significant. All reported outcomes include elective resection after colostomy construction, which was conducted in $94 \%$ of patients following colostomy creation. ASA classification could not be taken into account in this study, due to lack of reporting in the included articles.

Emergency surgery for LSCO is associated with a much higher mortality and morbidity rate, when compared to elective surgery. This is attributed to the advanced age, frequent comorbidities, reduced intake and progressive stage of disease. ${ }^{26}$ The use of a bridge to surgery approach using either stent or colostomy postpones the need for immediate resection. Hereby, time is created to optimize the patients' condition, let the (dilated) bowel restore and perform further tumor staging. ${ }^{27}$ When the patient is stabilized, elective resection of the tumor can be performed. The improved conditions means that laparoscopic surgery is often possible, as opposed to an open laparotomy approach, which is usually used in the emergency situation. ${ }^{28}$

This study underlines the relatively high morbidity and mortality rates reported in patients undergoing primary emergency resection for LSCO. ${ }^{7,29-31}$ Mortality and morbidity tended to be lower in the colostomy group and this is in accordance with data from previous cohort studies regarding colostomy as bridge to surgery. ${ }^{32}$ These results raise the question why colostomy as a bridge to surgery approach was abandoned in the past. Reasons for this included a prolonged hospital stay, increased number of surgical interventions and a lower quality of life reported 
for patients with a colostomy. ${ }^{31,33-36}$ However, the results of this systematic review show that significantly more primary anastomosis are created following resection in the colostomy group and fewer patients are left with a permanent colostomy. A recently published article by Young et al. demonstrated significant lower quality of life in patients treated with colostomy versus stent placement, which persisted up to 12 months after colonic decompression. However, in these patients, colostomies served as palliative treatment and therefore, none were reversed. ${ }^{37}$ Since the present study shows that significantly more patients will have to live with a permanent colostomy in the primary resection group, quality of life is more likely to be affected in this patient group versus when colostomy is constructed as bridge to surgery. In addition, total hospital stay seemed to be shorter in the colostomy group, which is probably accounted for by the improved surgical and minimally invasive techniques of current practice. Overall, these results may implicate that colostomy creation is a safe alternative and patients with malignant LSCO may benefit from this treatment modality, rather than primary resection.

Besides colostomy creation, stent placement can also be used as a bridge to elective surgery in patients with acute LSCO. Encouraged by good results from multiple retrospective studies on stent placement this seemed to be a very promising treatment option. However, the early closure of several randomized controlled trials investigating the role of stents in LSCO due to stent related complications has caused cautiousness towards this approach. ${ }^{11-13}$ Especially stent-related perforation is a feared complication. In the past years, several risk factors for stent-related colonic perforation were identified; such as total obstruction, length of the stricture $>40 \mathrm{~mm}$, benign disease and the use of an angiogenesis inhibitor. ${ }^{38-40}$ In addition to a possibly high complication rate, questions have been raised about the oncologic long-term results following stent placement, since it is thought that tumor manipulation by stent placement possibly leads to micro-perforations and tumor spill. However, only a few studies have reported on long-term outcomes. ${ }^{14,41,42} \mathrm{~A}$ recent systematic review recommended stent placement in palliative patients or when the patient is deemed unfit for major surgery only, which is in accordance with the latest European Guidelines for treatment of LSCO. ${ }^{38,43,44}$ Given the oncological uncertainties we agree that stent placement should be reserved for palliative patients and patients with a high surgical risk. In addition, we recommend that all palliative patients with one or more risk factors for stent-related colon perforation should also be treated with a colostomy, since stent perforation is a life-threatening complication that should be avoided at all cost. This means that colostomy creation is the only bridge to surgery option in curative patients and patients with risk factors for perforation.

This current study has several strengths. First, a meta-analysis is an important tool to highlight trends that may not be apparent in individual studies. Pooling data of similar studies increases precision and therefore the confidence level of the findings. Secondly, no publication bias was detected, which indicates that the pooled results may be unbiased.

However, there are several limitations of this study that should also be acknowledged. First, studies reporting on diverting colostomy as a bridge to surgery are limited. The outcomes of this systematic review and meta-analysis are therefore mainly based on comparative studies 
without a randomized design, while these would be preferred to obtain the highest level of evidence. Second, of the eight included studies four were published before 1995 and four studies reported on retrospective data, making them more prone to bias and confounding. Third, morbidity appeared to have been assessed differently in the different studies, therefore all complications were scored according to the surgical complication classification according to Clavien and Dindo. ${ }^{17}$ Although this classification is validated, it remains amendable to subjective bias. Also, no long-term complications were reported in the included studies; hereby, undesirable colostomy-related complications such as colostomy prolapse and parastomal herniation that occurred outside the 30-day morbidity range could have been missed. Finally, there were differences among studies in terms of surgical techniques for colostomy creation. However, this restriction was minimalized by conduction of a subgroup analysis of studies published after 1995 for the primary outcomes. All studies analyzed in this subgroup-analysis use minimally invasive techniques for colostomy creation.

This systematic review and meta-analysis provides an overview of all available literature on primary resection compared to colostomy creation followed by elective resection in patients with acute LSCO. Keeping the limitations of this study in mind, we conclude that a diverting colostomy as bridge to surgery may have several advantages over primary resection in patients with LSCO and should therefore be considered in patients with acute LSCO. Further prospective studies are warranted for the development of a patient tailored approach assigning patients with LSCO to the different treatment strategies. 


\section{REFERENCES}

1. Jullumstro E, Wibe A, Lydersen S, Edna TH. Colon cancer incidence, presentation, treatment and outcomes over 25 years. Colorectal Dis 2011;13:512-8

2. Winner M, Mooney SJ, Hershman DL, Feingold DL, Allendorf JD, Wright JD, Neugut Al. Incidence and predictors of bowel obstruction in elderly patients with stage IV colon cancer: a population-based cohort study. J Am Med Assoc Surg 2013;148:715-22

3. Scott NA, Jeacock J, Kingston RD. Risk factors in patients presenting as an emergency with colorectal cancer. $\mathrm{Br}$ J Surg 1995; Mar;82(3):321-3

4. Hennessey DB, Burke JP, Ni-Dhonochu T, Shields C, Winter DC, Mealy K. Preoperative hypoalbuminemia is an independent risk factor for the development of surgical site infection following gastrointestinal surgery: a multi-institutional study. Ann Surg 2010; Aug;252(2):325-9

5. Sjo OH, Larsen S, Lunde OC, Nesbakken A. Short term outcome after emergency and elective surgery for colon cancer. Colorectal Dis. 2009;11:733-739

6. Papadimitriou G, Manganas D, Phedias Georgiades C, Vougas V, Vardas K, Drakopoulos S. Emergency surgery for obstructing colorectal malignancy: prognostic and risk factors. J BUON 2015;20(2):406-12

7. Tan SG, Nambiar R. Resection and anastomosis of obstructed left colonic cancer: primary or staged?. Aust N Z J Surg 1995;65(10):728-31

8. Bosscher MR, van Leeuwen BL, Hoekstra HJ. Current management of surgical oncologic emergencies. PLOS One. 2015;10(5):e0124641

9. Huang X, Lv B, Zhang S, Meng L. Preoperative colonic stents versus emergency surgery for acute left-sided malignant colonic obstruction: a meta-analysis. J Gastrointest Surg, 2014;18 (3):584-91

10. Cirocchi R, Farinella E, Trastulli S, Desiderio J, Listorti C, Boselli C, Parisi A, Noya G, Sagar J. Safety and efficacy of endoscopic colonic stenting as a bridge to surgery in the management of intestinal obstruction due to left colon and rectal cancer: a systematic review and metaanalysis. Surg Oncol. 2013;22:14-21

11. van Hooft JE, Bemelman WA, Oldenburg B, Marinelli AW, Holzik MF, Grubben MJ, Sprangers MA, Dijkgraaf MG, Fockens P. Colonic stenting versus emergency surgery for acute left-sided malignant colonic obstruction: a multicentre randomised trial. Lancet Oncol 2011; 12: 344-352
12. Cheung HY, Chung CC, Tsang WW, Wong JC, Yau KK, Li MK. Endolaparoscopic approach vs conventional open surgery in the treatment of obstructing left-sided colon cancer: a randomized controlled trial. Arch Surg 2009; Dec;144(12):1127-32

13. Pirlet IA, Slim K, Kwiatkowski F, Michot F, Millat BL. Emergency preoperative stenting versus surgery for acute left-sided malignant colonic obstruction: a multicenter randomized controlled trial. Surg Endosc. 2011;25:1814-21

14. Sabbagh C, Browet F, Diouf M et al. Is Stenting as "a Bridge to Surgery" an Oncologically Safe Strategy for the Management of Acute, Left-Sided, Malignant, Colonic Obstruction?: A Comparative Study With a Propensity Score Analysis. Ann Surg 2013; 258:107-115

15. Moher D, Liberati A, Tetzlaff J, Altman DG. Preferred reporting items for systematic reviews and metaanalyses: the PRISMA statement. J Clin Epidemiol 2009; 62(10):1006-1012

16. Slim K, Nini E, Forestier D, Kwiatkowski F, Panis Y, Chipponi J. Methodological index for non-randomized studies (minors): development and validation of a new instrument. ANZ J Surg. 2003;73(9):712-6

17. Clavien PA, Barkun J, de Oliveira ML, Vauthey JN, Dindo D, Schulick RD, de Santibanes E, Pekolj J, Slankamenac K, Bassi C, Graf R, Vonlanthen R, Padbury R, Cameron $\mathrm{JL}$, Makuuchi M. The Clavien-Dindo classification of surgical complications: five-year experience. Ann Surg 2009;250(2):187-96

18. Higgins JP, Thompson SG, Deeks JJ, Altman DG. Measuring in- consistency in meta-analyses. BMJ 2003;327:557-560

19. Carson SN, Poticha SM, Shields TW. Carcinoma obstructing the left side of the colon. Arch Surg. 1977;112(4):523-6

20. Fielding LP, Stewart-Brown S, Blesovsky L. Large bowel obstruction caused by cancer: a prospective study. $B M J$ 1979;2:517-519

21. Gandrup P, Lund L, Balslev I. Surgical treatment of acute malignant large bowel obstruction. Eur J Surg 1992; 158: $427-430$

22. Huddy SP, Shorthouse AJ, Marks CG. The surgical treatment of intestinal obstruction due to left sided carcinoma of the colon. Ann $R$ Coll Surg Eng/ 1988;70(1):40-3 
23. Jiang JK, Lan YT, Lin TC, Chen WS, Yang SH, Wang HS, Chang SC, Lin JK. Primary vs. delayed resection for obstructive left-sided colorectal cancer: impact of surgery on patient outcome. Dis Colon Rectum. 2008;51(3):306-11

24. Kronborg O. Acute obstruction from tumour in the left colon without spread. A randomized trial of emergency colostomy versus resection. Int I Colorectal Dis. 1995;10(1):1-5

25. Tanis PJ, Paulino Pereira NR, van Hooft JE, Consten EC, Bemelman WA. Resection of Obstructive Left-Sided Colon Cancer at a National Level: A Prospective Analysis of Short-Term Outcomes in 1,816 Patients. Dig Surg. 2015;4;32(5):317-324

26. Runkel NS, Hinz U, Lehnert T, Buhr HJ, Herfarth C. Improved outcome after emergency surgery for cancer of the large intestine. Br J Surg 1998;85:1260-5

27. Small AJ, Coelho-Prabhu N, Baron TH. Endoscopic placement of self-expandable metal stents for malignant colonic obstruction: long-term outcomes and complication factors. Gastrointest Endosc. 2010;71(3):560-72

28. Quereshy FA, Poon JT, Law WL. Long-term outcome of stenting as a bridge to surgery for acute left-sided malignant colonic obstruction. Colorectal Dis. 2014;16(10):788-93

29. Sjo OH, Larsen S, Lunde OC, Nesbakken A. Short term outcome after emergency and elective surgery for colon cancer. Colorectal Dis 2009;11(7):733-9.

30. Anaraki F, Vafaie M, Behboo R, Maghsoodi N, Esmaeilpour S, Safaee A. Quality of life outcomes in patients living with stoma. Indian J Palliat Care. 2012;18(3):176-80

31. Vermeulen J, Gosselink MP, Busschbach JJ, Lange JF. Avoiding or reversing Hartmann's procedure provides improved quality of life after perforated diverticulitis. J Gastrointest Surg. 2010;14(4):651-7

32. Sommeling C.A., Haeck L. Caecostomy in the Management of Acute Left Colonic Obstruction. Acta chir belg, 1997, 97, 217-219

33. Park JJ, Del Pino A, Orsay CP, et al. Stoma complications: the Cook County Hospital experience. Dis Colon Rectum 1999; 42: 1575-80

34. Sprangers MA, Taal BG, Aaronson NK, te Velde A. Quality of life in colorectal cancer. Stoma vs. nonstoma patients. Dis Colon Rectum 1995; 38: 361-69

35. Cakmak A, Aylaz G, Kuzu MA. Permanent stoma not only affects patients' quality of life but also that of their spouses. World J Surg. 2010 Dec;34(12):2872-6
36. Small AJ, Coelho-Prabhu N, Baron TH. Endoscopic placement of self-expandable metal stents for malignant colonic obstruction: long-term outcomes and complication factors. Gastrointest Endosc 2010;71(3):560-72

37. Young CJ, De-Loyde KJ, Young JM, Solomon MJ, Chew $\mathrm{EH}$, Byrne CM, Salkeld G, Faragher IG. mproving Quality of Life for People with Incurable Large-Bowel Obstruction: Randomized Control Trial of Colonic Stent Insertion. Dis Colon Rectum. 2015;58(9):838-49

38. van Hooft JE, van Halsema EE, Vanbiervliet G, Beets-Tan RG, DeWitt JM, Donnellan F, Dumonceau JM, GlynneJones RG, Hassan C, Jiménez-Perez J, Meisner S, Muthusamy VR, Parker MC, Regimbeau JM, Sabbagh C, Sagar J, Tanis PJ, Vandervoort J, Webster GJ, Manes G, Barthet MA, Repici A. Self-expandable metal stents for obstructing colonic and extracolonic cancer: European Society of Gastrointestinal Endoscopy (ESGE) Clinical Guideline. Gastrointest Endosc. 2014;80(5):747-61.e175

39. Boyle DJ, Thorn C, Saini A, Elton C, Atkin GK, Mitchell IC, Lotzof K, Marcus A, Mathur P. Predictive factors for successful colonic stenting in acute large-bowe obstruction: a 15-year cohort analysis. Dis Colon Rectum. 2015;58(3):358-62

40. van Halsema EE, van Hooft JE, Small AJ, Baron $\mathrm{TH}$ García-Cano J, Cheon JH, Lee MS, Kwon SH, MucciHennekinne S, Fockens P, Dijkgraaf MG, Repici A. Perforation in colorectal stenting: a meta-analysis and a search for risk factors. Gastrointest Endosc. 2014;79(6):970-82

41. Erichsen $R$, Horváth-Puhó $E$, Jacobsen JB, Nilsson $T$, Baron JA, Sørensen HT. Long-term mortality and recurrence after colorectal cancer surgery with preoperative stenting: a Danish nationwide cohort study. Endoscopy. 2015;47(6):517-24

42. Sloothaak DA, van den Berg MW, Dijkgraaf MG, Fockens P, Tanis PJ, van Hooft JE, Bemelman WA collaborative Dutch Stent-In study group.Oncological outcome of malignant colonic obstruction in the Dutch Stent-In 2 trial. Br J Surg. 2014;101(13):1751-7

43. Zhoa X., Lio B., Zhao E., Wang J., Cai M., Xia Z., Xia Q., Shuai X., Tao K., Wang G., Cai K. The safety and efficiency of surgery with colonic stents in left-sided malignant colonic obstruction: a meta-analysis. Gastroenterol Res Pract. 2014:407325

44. Frago R, Ramirez E, Millan M, Kreisler E, del Valle E, Biondo $S$. Current management of acute malignant large bowel obstruction: a systematic review. Am J Surg 2014;207:127-3 



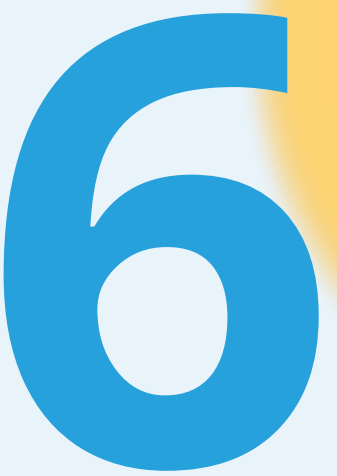

\title{
Efficacy of loop colostomy construction for acute left-sided colonic obstructions: a cohort analysis
}

International Journal of Colorectal Disease 2017

\author{
F.J. Amelung \\ C.L.J Mulder \\ E.C.J. Consten \\ W.A. Draaisma
}




\section{ABSTRACT}

\section{Background}

To investigate the safety of a loop colostomy in patients presenting with acute LSCO.

\section{Methods}

Retrospective analysis of all patients that received a loop colostomy for LSCO between 2003 and 2015. Primary outcomes were mortality, major morbidity (Clavien-Dindo grade III-IV) and minor morbidity (Clavien-Dindo grade I-II).

\section{Results}

146 patients presenting with acute LSCO received a diverting colostomy. After colostomy construction, mortality occurred in four patients $(2.7 \%)$ and major complications were reported in twenty patients (13.7\%). In 61 patients, the diverting colostomy served as a palliative measure, because of metastatic disease or unfitness for major surgery. The remaining 85 patients all underwent delayed resection, resulting in an overall mortality, major morbidity and minor morbidity of $6.9 \%(n=6), 14.0 \%(n=12)$ and $26.7 \%(n=23)$, respectively.

\section{Conclusions}

Diverting colostomy construction is a minimally invasive and safe treatment option for LSCO. It can serve as a definite palliative measure, as well as a bridge to elective surgery. A diverting colostomy as bridge to surgery might even be a valid alternative for emergency resections, since mortality and morbidity rates following colostomy construction and delayed resection appear lower than reported outcomes following primary resection. 


\section{INTRODUCTION}

In the Netherlands, around 15000 new patients present with colon cancer each year, making it one of the most common malignancies. ${ }^{1}$ Approximately $8-13 \%$ of these patients present with a colonic obstruction. ${ }^{2,3}$ The majority of these patients are elderly and in a poor clinical condition due to several days to weeks of reduced intake. In addition, presentation with acute colonic obstruction is associated with more advanced disease and a higher incidence of metastatic disease when caused by a malignancy. ${ }^{4-6}$ These factors all contribute to an increased peri- and postoperative surgical risk. On the other hand, left-sided colonic obstruction (LSCO) is considered a life-threatening condition that requires immediate intervention.

Primary resection is performed in the majority of patients presenting with LSCO in the Netherlands, even though this emergency procedure is associated with high mortality and morbidity rates. ${ }^{7,8}$ In addition, creation of a primary anastomosis is usually deemed unsafe due to severe bowel distension in this emergency setting. Because of this, many patients end up with a permanent colostomy, which is associated with frequent complications and a lower quality of life..$^{9-13}$

Minimally invasive salvage procedures can serve as a bridge to permanent cancer treatment while avoiding major surgery under poor conditions. Either placement of a Self-Expanding Metallic Stent (SEMS) or construction of a loop colostomy can be applied as an initial strategy to restore faecal passage with the objective to reduce bowel wall distension and to optimize the clinical condition prior to delayed resection. In addition, time is created for accurate tumour staging and neo-adjuvant treatment if necessary. ${ }^{14}$ Extensive surgery can hereby be avoided in patients with disseminated disease or unacceptable surgical risk.

Stenting as a bridge to surgery (BTS) has been proposed as an attractive alternative to emergency resection. A recent meta-analysis comparing SEMS as BTS with emergency resection showed significantly lower morbidity, mortality and long-term stoma rates for the SEMS group. ${ }^{7,15}$ However, SEMS as BTS in curable patients has been under debate since it has been suggested to worsen oncological outcomes and influence long-term survival. This could be attributed to tumour manipulation, possible perforation by passing the tumour with wires and by compressing the tumour with stents, which could lead to peritoneal tumour spill. ${ }^{16-19}$ No such risks are associated with the creation of a diverting colostomy. Therefore, this might be a safer alternative to primary resection rather than SEMS as BTS. However, literature on diverting colostomy as BTS is limited and randomized trials are lacking.

In our institution construction of a diverting colostomy has been the first treatment choice for LSCO, either as BTS or as a palliative procedure. To study the safety of diverting colostomy construction in patients with acute LSCO, we analysed mortality and morbidity rates for all patients that were treated with a colostomy between 2003-2015. 


\section{METHODS}

\section{Study design and population}

A retrospective cohort study was performed in a large teaching hospital in the Netherlands between 2003 and 2015. Hospital records were searched for all consecutive patients who presented with acute LSCO. Patients were considered to have LSCO when they had; 1 ) symptoms of bowel obstruction, such as absence of stool, abdominal pain, nausea and/or vomiting and 2) dilatation of the transverse colon, descending colon or rectum demonstrated on abdominal X-ray or computed tomography (CT). No direct evidence of a left-sided obstructing malignant tumour was required for inclusion. Patients treated with acute resection of the affected colon segment were excluded from further analysis.

We chose the timeframe 2003-2015 because from 2003 patient data was registered electronically, and starting from 2015 the hospital participated in the CONSTRUCT trial making colostomy creation no longer the standard of care for LSCOs.

\section{Outcome parameters}

Demographic characteristics (age, gender, ASA-score) and medical history were gathered from medical records. In addition, results of pre-operative examination (symptoms at presentation, findings at physical examination and radiological imaging), hospital stay, per-and postoperative morbidity and mortality were collected. Mortality or morbidity was defined as all events occurring within 30 days of the procedure. Complications were graded according to the classification of surgical complications developed and validated by Clavien and Dindo. ${ }^{20}$ Major morbidity was classified as grade III or higher, whereas minor morbidity was grade II or lower. Hospital stay was the total number of days the patient was admitted for during initial decompression, delayed resection and colostomy reversal (if conducted). Results were gathered and described for both primary colostomy construction and delayed resection if conducted. In addition, histological findings of biopsies were categorized according to the most advanced lesion. Described lesions were categorized by either adenocarcinoma, diverticulitis or other recorded non-neoplastic abnormalities.

\section{Surgical technique and sequel}

All included patients had a loop colostomy constructed on the right transverse colon. An incision in the right abdominal quadrant was made, the transverse colon was elevated to the skin and temporarily anchored with a plastic rod and fixed with resolvable stitches. After surgery, patients received enteral feeding as soon as possible. The eligibility for elective resection of the primary tumour was decided on after full diagnostic work-up consisting of a colonoscopy with histological biopsy and a CT-abdomen and/or MRI in case of rectal malignancy. In case of proven malignancy, additional imaging was performed to assess the presence of disseminated disease. Results were discussed within two weeks in a multidisciplinary oncological team consisting of surgeons, oncologists, radiologists and radiotherapists. Definitive treatment strategy was based upon consensus. Patients received either a delayed resection or a palliative approach in case of 
incurable disease or high surgical risk. Patients with resectable liver metastasis, or patients suited for HIPEC were not deemed incurable, but were considered for elective resection. When indicated, neo-adjuvant treatment was performed prior to elective resection. Finally, patients undergoing delayed resection were preferably operated laparoscopically with construction of a primary anastomosis. The diverting colostomy was only closed when deemed safe by the treating surgeon. BTS was defined as the step-up approach from primary colostomy construction to delayed resection, including all interim inquiries

\section{Statistical analysis}

Statistical analyses were performed using the Statistical Program for the Social Sciences (SPSS) version 21.0 (SPSS ${ }^{\circledR}$ Inc., Chicago, IL). Continuous variables were tested for normality using the Kolmogorov-Smirnoff test, and when normally distributed described as mean with standard deviation (SD). When continuous variables were non-normally distributed, they were described by medians with interquartile range (i.q.r.). Categorical variables were described as counts and percentages.

\section{RESULTS}

\section{Patient characteristics}

Between 2003 and 2015 a total of 154 patients presented with acute LSCO, as presented in Figure 1. Seven patients had to be excluded from analysis as they underwent primary resection as initial therapy for LSCO; five were diagnosed with peritonitis and suspected perforation necessitating emergency surgery and two were treated by acute resection because of untraceable reasons. One patient was excluded because she refused all further investigation and treatment.

Eventually 146 patients (62 male, 83 female) with a median age of 73.5 years (i.q.r. 62-82) were included in this study. An overview of all baseline and surgical characteristics is shown in Table 1.118 (80.8\%) patients underwent both plain abdominal X-ray and abdominal CT-scan prior to colostomy construction. The remaining patients underwent either an abdominal X-ray ( $n=13)$ or CT only ( $n=12)$. The site of obstruction was the splenic flexure in 8 patients, the descending colon in 19, the sigmoid in 94 , the rectosigmoid in 16 and the proximal rectum in 7 patients. 108 out of 146 left-sided obstructions were suspected for malignancy based on CT images.

\section{Diverting loop colostomy construction}

All colostomies were constructed at a median time interval of 1.5 days (0-14 days). Peritoneal metastases were encountered during surgery in sixteen patients (11.0\%). Four patients (2.7\%) died within 30 days after colostomy construction; all had an ASA-2 score and the cause of obstruction was colonic malignancy in three patients and benign disease in one. Cause of death was abdominal sepsis $(n=2)$, respiratory insufficiency after massive aspiration $(n=1)$ and unknown $(\mathrm{n}=1)$. Major morbidity occurred in 11 patients $(7.5 \%)$ and minor morbidity in 17 patients (11.6\%) (details in Table 2). 
FIGURE 1 | Flowchart of the study population

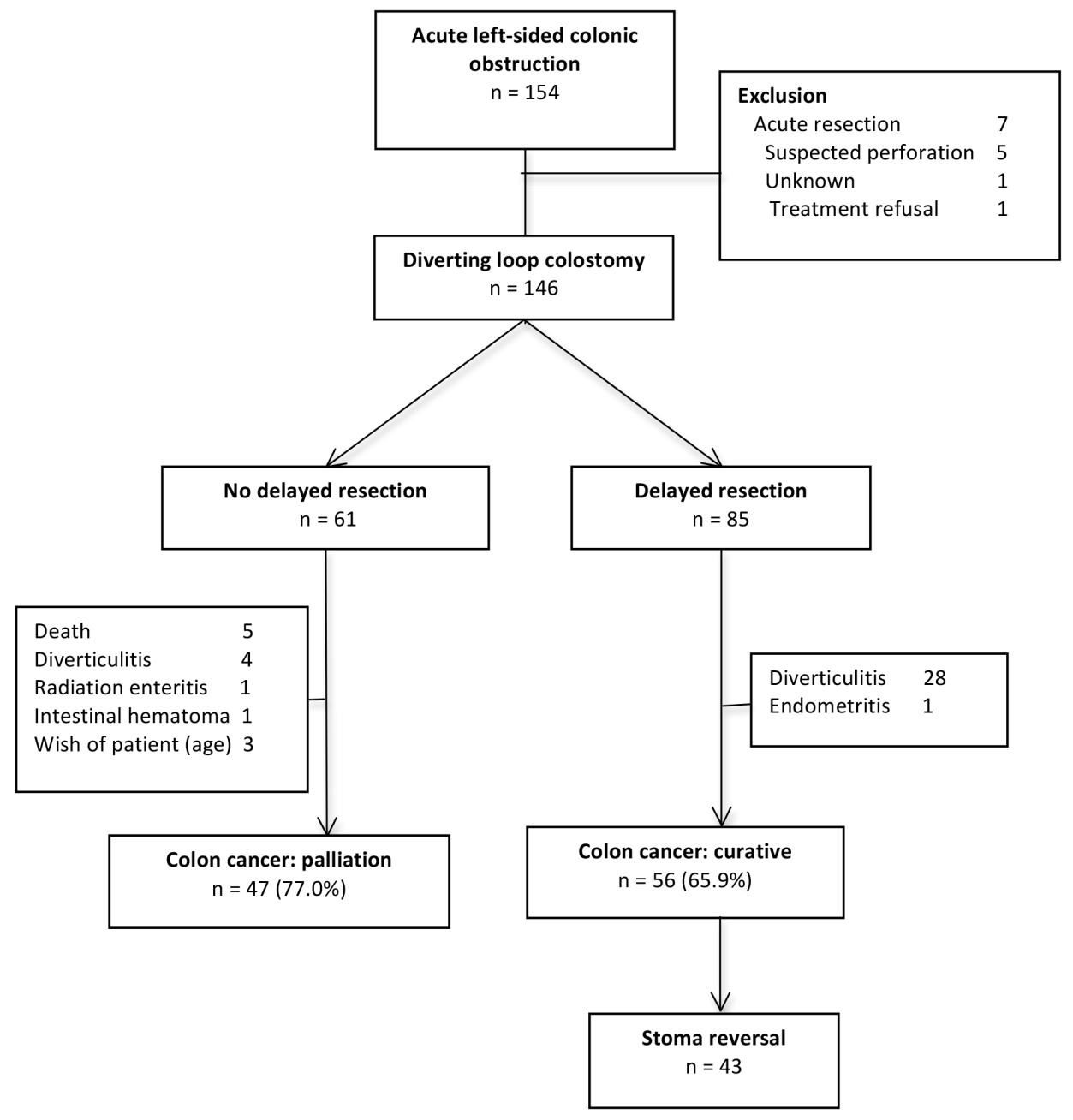

Eventually, colostomy construction served as a definite palliative measure in 61 patients. Reasons these patients did not undergo elective resection were; disseminated inoperable disease $(n=47)$, death after initial decompression $(N=5)$, diverticulitis $(n=4)$, radiation enteritis $(n=1)$, intestinal hematoma $(n=1)$ and according to the patients whish to refuse further treatment $(n=3)$. The remaining 85 patients were deemed to have curable disease and were fit enough to undergo delayed resection.

\section{Delayed resection}

85 patients underwent delayed resection of the affected colonic section. Three with proximal rectal cancer were given neo-adjuvant therapy prior to elective resection. Resection was 
TABLE 1 | Baseline and surgical characteristics for all patients treated with a deviating colostomy for a left-sided colonic obstruction at Meander Medical Centre from 2003-2015 ( $n=146)$

\begin{tabular}{|c|c|c|c|}
\hline & $\begin{array}{c}\text { Colostomy total } \\
\mathrm{n}(\%) \\
\mathrm{N}=146\end{array}$ & $\begin{array}{c}\text { Delayed resection } \\
\mathrm{n}(\%) \\
\mathrm{n}=85\end{array}$ & $\begin{array}{c}\text { No delayed } \\
\text { resection } n(\%) \\
n=61\end{array}$ \\
\hline Age in years & $71.4(13.8)$ & $68.3(12.6)$ & $75.8(14.4)$ \\
\hline Gender male:female & $63: 83$ & $35: 50$ & $28: 33$ \\
\hline \multicolumn{4}{|l|}{ ASA classification } \\
\hline 1 & $50(34.2)$ & $35(41.2)$ & $15(24.6)$ \\
\hline II & $79(54.1)$ & $44(51.8)$ & $35(57.4)$ \\
\hline III & 17 (11.6) & $6(7.1)$ & $11(18.0)$ \\
\hline Bowel surgery in past & $11(7.5)$ & $7(8.3)$ & $4(6.9)$ \\
\hline \multicolumn{4}{|l|}{ Symptoms at presentation } \\
\hline Obstipation & $123(84.2)$ & $71(83.5)$ & $52(85.3)$ \\
\hline Mean days of obstipation & $5(4-7)$ & $5(4-7)$ & $5(4-7)$ \\
\hline Nausea & $107(73.3)$ & $66(79.5)$ & $41(67.2)$ \\
\hline Vomiting & $87(59.6)$ & $55(66.3)$ & $32(52.2)$ \\
\hline Abdominal pain & $125(85.6)$ & $80(95.2)$ & $45(75.4)$ \\
\hline Rectal blood loss & $8(5.5)$ & $2(2.4)$ & $6(9.8)$ \\
\hline Weight loss (>3kg in 1 month) & $28(19.2)$ & $18(21.4)$ & $10(16.4)$ \\
\hline Fever $(>38 \mathrm{C})$ & $7(4.5)$ & $4(4.8)$ & $3(4.9)$ \\
\hline \multicolumn{4}{|l|}{ Biochemistry } \\
\hline C-reactive protein & $26(9-70)$ & $18(8-67)$ & $36(12.8-92.5)$ \\
\hline White blood cell count $t_{b}$ & $11.0(8.5-14)$ & $11.3(8.9-14.1)$ & $10.5(7.8-13.6)$ \\
\hline Hemoglobin $_{a}$ & $8.7(4.9)$ & $8.5(1.3)$ & $8.0(1.3)$ \\
\hline \multicolumn{4}{|l|}{ Pre-operative abdominal examination } \\
\hline Plain abdominal X-ray & $132(90.4)$ & $78(91.8)$ & $54(88.5)$ \\
\hline CT-scan & $132(90.4)$ & 79 (92.9) & $53(86.9)$ \\
\hline \multicolumn{4}{|l|}{ Tumor location } \\
\hline Splenic flexure & $10(6.4)$ & $5(5.9)$ & $5(8.2)$ \\
\hline Descending colon & $19(13.0)$ & $11(12.9)$ & $8(13.1)$ \\
\hline Sigmoid & $94(64.4)$ & $63(74.1)$ & $31(50.8)$ \\
\hline Rectosigmoid & $16(11.0)$ & $5(5.9)$ & $11(18.0)$ \\
\hline Proximal rectum & $7(4.8)$ & $1(1.2)$ & $6(9.8)$ \\
\hline \multicolumn{4}{|l|}{ Cause of obstruction } \\
\hline Benign disease & $35(24.0)$ & $29(34.1)$ & $6(9.8)$ \\
\hline Malignant disease & $108(74.0)$ & $56(65.9)$ & $52(85.3)$ \\
\hline Histologically proven colon carcinoma & - & $56(100 \%)$ & - \\
\hline Days to resection§ & - & - & $25(17-47)$ \\
\hline \multicolumn{4}{|l|}{ Resection type } \\
\hline Left-hemicolectomy & - & $13(15.3)$ & - \\
\hline Sigmoidectomy & - & $47(55.3)$ & - \\
\hline Sub-total colectomy & - & $3(3.5)$ & - \\
\hline LAR/rectosigmoidectomy & - & $22(25.9)$ & - \\
\hline Laparoscopic surgery & - & $40(47.1)$ & - \\
\hline Conversion & - & $10(25.0)$ & - \\
\hline
\end{tabular}

Values are ${ }_{\mathrm{a}}$ means (SD) and ${ }_{\mathrm{b}}$ median (inter quartile range). Abbreviations; ASA: American Society of Anesthesiologists.

performed after a median time interval of 25 days (i.q.r. 17-47) after colostomy construction. There were 10 low anterior resections, 12 rectosigmoidectomies, 47 sigmoidectomies, 13 left hemicolectomies and 3 (sub)total colectomies. 40 patients $(47.1 \%)$ had laparoscopic resection 
TABLE 2 | Colostomy construction related complications in the entire colostomy cohort $(n=146)$

\begin{tabular}{|c|c|c|c|c|}
\hline Complications n (\%) & $\begin{array}{c}\text { After } \\
\text { colostomy } \\
\text { construction } \\
\text { only }\end{array}$ & $\begin{array}{c}\text { Colostomy as } \\
\text { BTS after } \\
\text { colostomy } \\
\text { construction } \\
\text { only }\end{array}$ & $\begin{array}{c}\text { Colostomy as } \\
\text { BTS after } \\
\text { delayed } \\
\text { resection }\end{array}$ & $\begin{array}{l}\text { Colostomy } \\
\text { as BTS total }\end{array}$ \\
\hline & $n=146$ & $n=85$ & $n=85$ & $n=85$ \\
\hline Mortality & $4(2.7)$ & - & $2(2.4)$ & $6(6.9)$ \\
\hline \multicolumn{5}{|l|}{ Cause of death } \\
\hline Abdominal sepsis & $2(1.4)$ & - & $2(2.4)$ & $4.7(4.7)$ \\
\hline Respiratory insufficiency & $1(0.7)$ & - & - & $1(1.2)$ \\
\hline Unknown & $1(0.7)$ & - & - & $1(1.2)$ \\
\hline Major morbidity & $11(7.5)$ & $6(7.1)$ & $8(9.4)$ & $12(14.0)_{b}$ \\
\hline Abdominal dehiscence & $1(0.7)$ & - & - & - \\
\hline Intestinal ischemia & $2(1.4)$ & $2(2.4)$ & - & $2(2.3)$ \\
\hline Postoperative bleeding & $1(0.7)$ & - & $2(2.4)$ & $1(1.2)$ \\
\hline Surgical intra-abdominal abscess drainage & $1(0.7)$ & - & $1(1.2)$ & $1(1.2)$ \\
\hline Abdominal sepsis with exploratory laparotomy & $3(2.1)$ & $1(1.2)$ & $5(5.9)$ & $5(5.8)$ \\
\hline Stoma revision & $2(1.4)$ & $2(2.4)$ & - & $2(2.3)$ \\
\hline Respiratory insufficiency & $1(0.7)$ & $1(1.2)$ & - & $1(1.2)$ \\
\hline Minor morbidity & $6(9.8)$ & $8(9.4)$ & $21(24.7)$ & $23(26.7)_{b}$ \\
\hline Wound infection & $2(3.3)$ & $4(4.7)$ & $12(14.1)$ & $12(26.3)$ \\
\hline Urinary tract infection & $4(6.6)$ & $2(2.4)$ & $5(5.9)$ & $5(5.8)$ \\
\hline Pneumonia & $2(3.3)$ & $3(3.3)$ & $6(7.1)$ & $6(7.0)$ \\
\hline Permanent colostomy & - & - & - & $16(18.8)$ \\
\hline Hospital stay in days ${ }_{c}$ & & & & $17(14-22)$ \\
\hline
\end{tabular}

${ }_{a}$ Mortality includes all patients that died within 30 days after colostomy creation, since it was impossible to recover whether they would have undergone elective resection. ${ }_{b}$ Total outcomes are not the sum of the previous two columns since some patients had more than one complication. ${ }_{c}$ Hospital stay is reported in days and includes hospital stay for construction of the colostomy, delayed resection and colostomy reversal (if conducted), it is reported in median (interquartile range). Abbrevations; BTS: bridge to surgery.

of which 10 had to be converted to an open procedure due to extensive adhesions $(n=7)$, obesity $(n=1)$, ureter damage $(n=1)$ and advanced disease $(n=1)$. Laparoscopic resection was not performed in any patients operated on from 2003-2007. In the following years, the number of patients operated on laparoscopically increased annually up to 75\% in 2014.

Mortality occurred in 2 patients (2.4\%) after delayed resection due to intra-abdominal bleeding of unknown origin $(n=1)$ and abdominal sepsis $(n=1)$. Major morbidity was encountered in 8 patients (9.4\%); postoperative bleeding needing surgical re-intervention $(n=2)$, abscess formation requiring percutaneous drainage $(n=1)$ and exploratory laparotomy for abdominal sepsis ( $n=5) .21$ patients (24.7\%) had minor morbidity consisting of wound infection $(n=10)$, pneumonia $(n=5)$ and urinary tract infection $(n=6)$ (Table 2).

To provide a complete overview, total mortality and morbidity rates for the BTS group after the initial colostomy creation and delayed resection combined are shown in Table 2 . The overall mortality rate includes all patients that died after initial colostomy construction, as well as after elective resection. Of the four patients that died after colostomy construction, three 
TABLE 3 | TNM staging for resected specimens with malignant disease $(n=55)$

\begin{tabular}{ccc}
\hline Stage & TNM & Number (\%) \\
\hline O & Tis, N0, M0 & $0(0)$ \\
I & T1-2, N0, M0 & $3(5.4)$ \\
II & T3-4, N0, M0 & $14(25.5)$ \\
III & T1-4, N1, M0 & $22(40.0)$ \\
IV & T-14, N1-2, M1 & $16(29.1)$ \\
\hline
\end{tabular}

presented with disseminated disease. Colostomy would most likely have served as a palliative measure in these patients. However, since no additional staging was performed, it is impossible to determine with certainty that they would not have undergone elective resection. In order to present the most objective data, these patients were therefore all included in the overall mortality rate of the BTS group. Therefore, overall mortality in the BTS group following colostomy construction and delayed resection combined was $6.9 \%(n=6)$. Major morbidity occurred in 12 patients (14.0\%) and minor morbidity in 23 patients (26.7\%). Median hospital stay was 17 days (i.q.r. 14-22), which includes hospital stay for all conducted procedures.

\section{Stoma reversal pathology results}

Stoma reversal was possible in 69 of 146 patients (47.2\%). Of the patients that received a delayed resection after colostomy construction $(n=85)$, only 16 patients did not have their colostomy reversed (18.8\%). In 15 patients (22.1\%) the colostomy was reversed during the delayed resection surgery, whereas the other 54 patients $(n=78.3 \%)$ had their colostomy reversed in a third operation. Histological analysis of the 85 resected specimens demonstrated malignancy in 56 patients, diverticulitis in 28 and endometriosis in 1 patient. TNM staging showed that $67.9 \%$ $(n=38)$ of the patients with malignant disease had stadium III-IV colon carcinoma (Table 3). Five patients underwent additional liver metastatectomy after delayed resection.

\section{DISCUSSION}

This study investigates the safety of diverting colostomy construction with the intention to decompress the colon for LSCO in either a curative or palliative setting. The results of our study implicate that colostomy creation for acute left-sided colonic obstruction is a safe treatment option. Mortality and morbidity rates are relatively low for both palliative patients, as well as patients that had the colostomy constructed as a bridge to surgery and underwent subsequent elective resection.

In total, 85 patients (58.2\%) underwent a delayed resection after multidisciplinary oncological discussion. The remaining 61 patients kept their colostomy as a palliative measure and were not exposed to a colonic resection due to lack of perspective on cure, high surgical risk or according to the patients' wishes. Treatment algorithms for LSCOs differ depending on whether patients are treated with curative or palliative intend. Below we will therefore separately discuss possible treatment options for curative and palliative patients. 
Primary resection is currently the most performed treatment option in LSCO patients, but the construction of a diverting colostomy as BTS has several important advantages over this approach. Stoma construction is a relatively minor intervention that leads to immediate decompression of the colon, whilst postponing the need for emergency resection. In the current study, patients that did undergo an elective resection had their surgery performed a median of 25 days (17-47) after initial decompression. This time was used to optimize the patients' condition and let the bowel recover to its original state. Hereby, more favourable circumstances for colonic resection were created, making laparoscopic surgery more often an option. ${ }^{20}$ In the present study, 40 patients (47.1\%) underwent a laparoscopic procedure. However, laparoscopic resections weren't carried out until 2008 at our institution; when all patients operated on before 2008 are excluded from analyses, laparoscopic resection rate increases to $58.8 \%$ (40 out of 68 patients). The main goal of optimizing the patients' condition is, of course, reducing mortality and complication rate. Indeed, when we compare the outcomes of our 85 patients whom received a colostomy as BTS with outcomes reported for primary resection, overall complication, major morbidity and mortality rate are much higher in the primary resection group ( $26.7 \%$ vs. $29-52 \%, 14 \%$ vs. $19-28 \%$ and $7 \%$ vs. $10-24 \%$, respectively)..$^{21-25}$ In addition, a recent meta-analysis reported that approximately $30 \%$ of the patients treated with acute resection ends up with a permanent colostomy, compared to only $18.8 \%(n=16)$ in the present study. Comparable to our study, all included patients in the metaanalysis were treated with curative intent. ${ }^{7}$ This is an important finding, since a main reason surgeons use colostomy as BTS with reserve is because a permanent colostomy is associated with a lower quality of life. ${ }^{13,25}$ However, our results suggest that patients treated with a colostomy as BTS might be less likely to end up with a permanent colostomy compared to patients treated with primary resection; quality of life might therefore even be better in patients treated with a colostomy as BTS. Overall, colostomy construction as bridge to surgery seems to result in more laparoscopic resections, lower complication rates, fewer permanent stomas and lower mortality rates compared to results reported for primary resection. Disadvantages of stoma construction are the need for multiple surgeries and a high risk of incisional hernia rate following stoma reversal. ${ }^{26} \mathrm{~A}$ recent population-based analysis showed mortality and morbidity rates following acute resection for LSCO to be specifically increased in elderly frail patients (age $\geq 70$ years and/or ASA-score $\geq 3$ ). ${ }^{8}$ Therefore, a bridge to surgery approach might be especially beneficial in these high-risk patients. ${ }^{27}$

Apart from stoma construction, SEMS placement can also serve as a bridge to surgery approach. SEMS placement as BTS has been studied extensively in recent years. Encouraged by good short-term results seen in a recent meta-analysis comparing SEMS with acute resection, SEMS seemed a promising treatment option. ${ }^{7}$ However, recent studies show that stent placement might lead to worsening of long-term survival and higher local recurrence rates. ${ }^{18-22}$ Since the effects on long-term outcomes are still uncertain, the use of SEMS for acute LSCO is currently limited to incurable patients or patients with a high surgical risk (age $\geq 70$ or ASA-score $\geq 3$ ). ${ }^{27}$ Stoma construction does not seem to have any negative effects on oncological outcomes. ${ }^{28}$ In addition, it is important to realize that benign disease is a known risk factor for stent 
perforation, making SEMS an inapplicable treatment option in these patients as well. ${ }^{29}$ In $21.2 \%$ ( $n=31$ ) of the patients included in the current study diverticulitis was the underlying cause of the obstruction, $89 \%$ ( $n=27$ ) of these patients eventually underwent elective resection. The overall reasonably low mortality and morbidity rates presented in the present study imply that colostomy construction could serve as a safe treatment option for patients with benign disease as well. Therefore, colostomy construction appears to be the only BTS approach possible for fit curative patients and patients with benign disease.

For palliative patients, treatment goals are different and preserving quality of life has a great influence on treatment choice. Currently, the vast majority of patients with a LSCO are treated with primary resection. For palliative patients, this means they undergo an unnecessary major abdominal surgery, which could have been prevented if stent placement or stoma construction were used as initial decompression. Both stent and stoma provide time for adequate oncologic staging and, when the patient is deemed inoperable, can both serve as a definite palliative measure. A possible disadvantage of colostomy as treatment for palliative patients is that they will have their colostomy until death, which is associated with a poorer quality of life. ${ }^{10-12} \mathrm{~A}$ recent RCT by Young et al. (2015) showed a significant and persistent better quality of life in patients treated with SEMS as a palliative procedure versus emergency surgery (either colostomy or acute resection). ${ }^{24}$ This study indicates that palliative patients could be better off with SEMS rather than a colostomy for their final stage of life. We would therefore recommend SEMS placement to be the first choice of treatment for patients with preoperatively proven disseminated disease.

Prior studies focusing on diverting colostomy as treatment option for LSCOs are limited and of older date. One randomized trial dating from 1995 can be identified that reports on this approach. ${ }^{30}$ Unfortunately, this study suffers from severe methodological flaws and conclusions can therefore not be drawn solely based on this study. In addition to this trial, five retrospective studies and two prospective studies have been published demonstrating that diverting colostomy for acute LSCO can be safely executed. Although promising, these studies have failed to gain strong evidence due to poor study design and the fact that patients were only studied following the delayed resection and not after initial stoma construction. ${ }^{28,31-37}$ In the current study, these outcomes are included. In addition, modern minimally invasive surgical techniques were used, making the outcomes applicable to the current clinical practice. However, some limitations should be kept in mind, such as its retrospective design and the lack of a control group. Unfortunately, since acute resection and/or SEMS placement are not performed as a treatment for LSCO in our centre it was not possible to realise a comparative analysis. Possible selection bias introduced by the use of retrospective data was minimalized, since all consecutive patients that presented with acute LSCO from 2003-2015 were treated with colostomy construction, as this is the standard of care. Also, the relatively high number of patients included in this cohort strengthens the outcomes. We chose to include benign as well as malignant disease, because in the acute setting, treatment algorithm for a colonic obstruction is similar for all patients, regardless of aetiology. Certainty about the underlying 
cause of obstruction can only be fully provided by histological review of the resected colon segment; therefore all patients fit for surgery eventually undergo a full oncologic resection. Even though inclusion of different underlying causes might lead to a more heterogenic patient group, results remain applicable in general practice since treatment algorithms do not differ. In addition, subgroup analysis of patients with diverticulitis vs. malignancy as underlying cause did not reveal any difference regarding mortality and morbidity; therefore inclusion of these patients probably did not influence the overall results.

Keeping the limitations of this study in mind, we conclude that construction of a loop colostomy for acute LSCO is a safe procedure with promisingly low morbidity and mortality rates, which leads to a high percentage of colostomy reversal. Overall, we would recommend primary resection for all curative patients $\leq 70$ years and with a low surgical risk, since morbidity and mortality rates are relatively low for these patients. For all curative patients with an increased surgical risk (age $\geq 70$ and/or ASA-score $\geq 3$ ) and all patients with a benign obstruction we would recommend stoma construction, since the oncologic outcomes of SEMS placement remain uncertain and benign strictures are a known risk factor for SEMS-related perforation. Stent should be considered first choice in all palliative patients, since this treatment approach has the smallest effect on quality of life. 


\section{REFERENCES}

1. Dutch cancer registration. http://www.cijfersoverkanker. nl/selecties/Dataset_1/img54e494ab18fdf. Accessed September $7^{\text {th }}, 2015$

2. Jullumstro E, Wibe A, Lydersen S, Adna TH. Colon cancer incidence, presentation, treatment and outcomes over 25 years. Colorectal Dis 2011;13:512-8

3. Winner M, Mooney SJ, Hershman DL, Hershman DL, Feingold DL, Allendorf JD, Wright JD, Neugut AI. Incidence and predictors of bowel obstruction in elderly patients with stage IV colon cancer: a population-based cohort study. J Am Med Assoc Surg 2013;148:715-22

4. Scott NA, Jeacock J, Kingston RD. Risk factors in patients presenting as an emergency with colorectal cancer. $\mathrm{Br}$ J Surg 1995;82(3):321-3

5. Hennessey DB, Burke JP, Ni-Dhonochu T, Shields C, Winter DC, Mealy K. Preoperative hypoalbuminemia is an independent risk factor for the development of surgical site infection following gastrointestinal surgery: a multi-institutional study. Ann Surg 2010;252(2):325-9

6. Smothers L, Hynan L, Fleming J, Turnage R, Simmang C, Anthony T. Emergency surgery for colon carcinoma. Dis Colon Rectum 2003;46(1):24-30

7. Huang X, Lv B, Zhang S, Meng L. Preoperative colonic stents versus emergency surgery for acute left-sided malignant colonic obstruction: a meta-analysis. J Gastrointest Surg. 2014;18(3):584-91

8. Tanis PJ, Paulino Pereira NR, van Hooft JE, Consten ECJ, Bemelman WA. Resection of Obstructive Left-Sided Colon Cancer at a National Level: A Prospective Analysis of Short-Term Outcomes in 1,816 Patients. Dig Surg. 2015;4;32(5):317-324

9. Mealy K, O'Brian E, Donohue J, Tanner A, Kaene FB. Reversible colostomy: what is the outcome? Dis Colon Rectum 1996;39:1227-1231

10. Mauro MA, Koehler RE, Baron TH. Advances in gastrointestinal intervention: the treatment of gastroduodenal and colorectal obstruc- tions with metallic stents. Radiology 2000;215:659-669

11. Nugent KP, Daniels $P$, Stewart B, Patankar R, Johnson CD. Quality of life in stoma patients. Dis Colon Rectum 1999;42: 1569-74

12. Park JJ, Del Pino A, Orsay CP, Nelson RL, Pearl RK, Cintron JR, Abcarian H. Stoma complications: the Cook County Hospital experience. Dis Colon Rectum 1999;42: 1575-80
13. Sprangers MA, Taal BG, Aaronson NK, te Velde A. Quality of life in colorectal cancer. Stoma vs. nonstoma patients. Dis Colon Rectum 995;38: 361-69

14. Bosscher MR, van Leeuwen BL, Hoekstra HJ. Current management of surgical oncologic emergencies. PLOS One. 2015;1;10(5):e0124641

15. Zhao XD, Cai BB, Cao RS, Shi RH. Palliative treatment for incurable malignant colorectal obstructions: a metaanalysis. World J Gastroenterol 2013;7;19(33):5565-74

16. Maruthachalam K, Lash GE, Shenton BK, Horgan AF. Tumour cell dissemination following endoscopic stent insertion. Br J Surg 2007;94:1151-1154

17. Erichsen R, Horváth-Puhó E, Jacobsen JB, Nilsson $T$, Baron JA, Sørensen HT (2015) Long-term mortality and recurrence after colorectal cancer surgery with preoperative stenting: a Danish nationwide cohort study. Endoscopy 47(6):517-24

18. Sloothaak DA, van den Berg MW, Dijkgraaf MG, Fockens P, Tanis PJ, van Hooft JE, Bemelman WA. Oncological outcome of malignant colonic obstruction in the Dutch Stent-In 2 trial. Br J Surg. 2014;101(13):1751-7

19. Sabbagh C, Browet F, Diouf M, Cosse C, Brehant O, Bartoli E, Mauvais F, Chauffert B, Dupas JL, Nguyen-Khac E, Regumbeau JM. Is Stenting as "a Bridge to Surgery" an Oncologically Safe Strategy for the Management of Acute, Left-Sided, Malignant, Colonic Obstruction?: A Comparative Study With a Propensity Score Analysis. Ann Surg 2013;258:107-115

20. Quereshy FA, Poon JT, Law WL. Long-term outcome of stenting as a bridge to surgery for acute left-sided malignant colonic obstruction. Colorectal Dis 2014;16(10):788-93

21. Clavien PA, Barkun J, de Oliveira ML, Vauthey JN, Dindo D, Schulick RD, de Santibanes E, Pekoli J, Slankamenac K, Basse C, Graf R, Vonlanthen R, Padbury R, Cameron $\mathrm{JL}$, Makuuchi M. The Clavien-Dindo classification of surgical complications: five-year experience. Ann Surg 2009;250(2):187-196

22. Kolfschoten NE, Wouters MWJM, Gooiker GA, Eddes $\mathrm{EH}$, Kievit J, Tollenaar RA, Marang-van de Mheen PJ. Nonelective Colon Cancer Resections in Elderly Patients: Results from the Dutch Surgical Colorectal Audit. Dig Surg 2012;29:412-419

23. Hooft JE, Bemelman WA, Oldenburg B, Marinelli AW, Lutke Holzik MF, Grubben MJ, Sprangers MA, Dijkgraaf MG, Fockens P. Colonic stenting versus emergency surgery for acute left-sided malignant colonic obstruction: a multicentre randomised trial. Lancet Oncol 2011;12(4):344-352 
24. Martinez-Santos C, Lobato RF, Fradejas JM, Pinto I, Ortega-Debalion P, Moreno-Azcoita M. Self-expandable stent before elective surgery vs. emergency surgery for the treatment of malignant colorectal obstructions: comparison of primary anastomosis and morbidity rates. Dis Colon Rectum 2002;45: 401-06

25. Young CJ, De-Loyde KJ, Young JM, Solomon MJ, Chew EH, Byrne CM, Salkeld G, Faragher IG. Improving Quality of Life for People with Incurable Large-Bowel Obstruction: Randomized Control Trial of Colonic Stent Insertion. Dis Colon Rectum 2015;58(9):838-49

26. Bhangu A, Nepogodiev D, Futaba K. Systematic review and meta-analysis of the incidence of incisional hernia at the site of stoma closure. World J Surg 2012;36:973983

27. Richtlijn colorectaal carcinoom 2013. Integraal Kanker Centrum Nederland http://oncoline.nl/uploaded/docs/ Colorectaal\%20carcinoom/Conceptrichtlijn\%20 Colorectaal\%20carcinoom.pdf Accessed at October $20^{\text {th }}$ 2015

28. Jiang JK, Lan YT, Lin TC, Chen WS, Yang SH, Wang HS, Chang SC, Lin JK. Primary vs. delayed resection for obstructive left-sided colorectal cancer: impact of surgery on patient outcome. Dis Colon Rectum 2008;51(3):306-11

29. van Halsema EE, van Hooft JE, Small AJ, Baron TH, García-Cano J, Cheon JH, Lee MS, Kwon SH, MucciHennekinne S, Fockens P, Dijkgraaf MG, Repici A. Perforation in colorectal stenting: a meta-analysis and a search for risk factors. Gastrointest Endosc 2014;79(6):970-82.e7
30. Kronborg. Acute obstruction from tumour in the left colon without spread. A randomised trial of emergency colostomy versus resection. Int. I Colorectal Dis $1995 ; 10-1-5$

31. Amelung FJ, Mulder CLJ, Verheijen PM, Draaisma WA, Siersema PD, Consten ECJ. Acute resection versus bridge to surgery with diverting colostomy for patients with acute malignant left-sided colonic obstruction: systematic review and meta-analysis. Surgical Oncology 2015;24 313-321

32. Fielding LP, Stewart-Brown S, Blesovsky L. Large bowel obstruction caused by cancer: a prospective study. BMJ 1997;2:517-519

33. Gandrup P, Lund L, Balslev I. Surgical treatment of acute malignant large bowel obstruction. Eur J Surg 1992;158:427-430

34. Huddy SP, Shorthouse AJ, Marks CG. The surgical treatment of intestinal obstruction due to left sided carcinoma of the colon. Ann $R$ Coll Surg Engl 1988;70(1):40-3

35. Ragland JJ, Londe AM, Spratt JS Jr. Correlation of the prognosis of obstructing colorectal carcinoma with clinical and pathologic variables. Am J Surg 1971;121(5):552-6

36. Tan SG, Nambiar R. Resection and anastomosis of obstructed left colonic cancer: primary or staged?.Aust N Z J Surg 1995;65(10):728-31

37. Carson SN, Poticha SM, Shields TW. Carcinoma obstructing the left side of the colon. Arch Surg 1997;112(4):523-6 



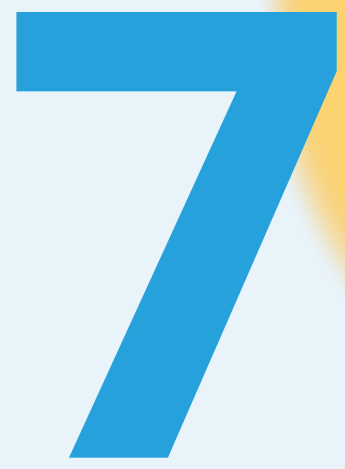

Deviating colostomy vs stent placement as bridge to surgery for malignant left-sided colonic obstruction

Surgical Endoscopy 2016

F.J. Amelung

F. ter Borg

E.C.J. Consten

P.D. Siersema

W.A. Draaisma 


\section{ABSTRACT}

\section{Background}

Acute colonic decompression using a deviating colostomy (DC) or a self-expandable metal stent (SEMS) has been shown to lead to fewer complications and permanent stomas compared to acute resection in elderly patients with malignant left-sided colonic obstruction (LSCO). However, no consensus exists on which decompression method is superior, especially in patients treated with curative intend. This retrospective study therefore aimed to compare both decompression methods in potentially curable LSCO patients.

\section{Methods}

All LSCO patients treated with curative intent between 2004-2013 in two teaching hospitals were retrospectively identified. In one institution, a DC was the standard of care, whereas in the other all patients were treated with SEMS.

\section{Results}

In total 88 eligible LSCO patients with limited disease and curative treatment options were included; 51 patients had a SEMS placed and 37 patients a DC constructed. All patients eventually underwent a subsequent elective resection. 235 patients were excluded due to benign or inoperable disease. No significant differences were found for hospital stay, morbidity, disease free and overall survival and mortality. Major complications were seen in 13/51 (25.5\%) patients in the SEMS group, and were mostly due to stent dysfunction $(n=7)$. Also, one stentrelated perforation occurred. Major complications occurred in 4/37 (10.8\%) patients in the DC group, including abdominal sepsis $(n=3)$ and wound dehiscence $(n=1)$. Long-term complication rate was significantly higher in the DC group ( $29.7 \%$ vs. $9.8 \%, p=0.01)$, mainly due to a high incisional hernia rate. Fewer patients had a temporary colostomy following elective resection after SEMS placement (62.2\% vs. $17.6 \%, \mathrm{p}<0.01)$. Permanent colostomy rate was not significantly different.

\section{Conclusion}

SEMS and DC are both effective decompression methods for curable LSCO patients with comparable short- and long-term oncological outcomes; however more surgical procedures are performed after DC due to an increased number of temporary colostomies and incisional hernia repairs. 


\section{INTRODUCTION}

Approximately one-tenth of patients with colorectal cancer (CRC) presents with an acute obstruction..$^{1,2}$ These patients are often elderly and in a poor general condition after a period of reduced intake with significant weight loss..$^{3,4}$ In addition, patients presenting with an acute obstruction are known to have more advanced disease. ${ }^{5,6}$ These circumstances all contribute to an increased surgical risk, especially in the acute situation. Also, the ability to conduct a proper oncologic resection, which is vital for the long-term prognosis, is technically demanding due to a severely distended bowel.

Nevertheless, acute colonic obstructions are usually treated with primary resection in the Netherlands, even though it is associated with morbidity and mortality rates up to $60 \%$ and $22 \%$, respectively. ${ }^{6,8} \mathrm{~A}$ recently conducted national audit reported slightly better outcomes ( $6.9 \%$ mortality in patients $<70$ years), but mortality still remains as high as $32.2 \%$ in high-risk elderly patients. ${ }^{9}$ In addition, many patients end up with a permanent colostomy, which adversely affects quality of life..$^{10,11}$

Colonic decompression is increasingly considered an appropriate alternative to acute resection. The above-mentioned national audit has also shown lower morbidity and mortality rates when colonic decompression was performed as bridge to elective surgery (BTS). ${ }^{9}$ Acute decompression can be achieved by constructing a deviating colostomy (DC) or by placement of a selfexpandable metallic stent (SEMS). Both approaches restore fecal passage, hereby avoiding the immediate risk of perforation and creating time to optimize the patients' clinical condition prior to elective resection. ${ }^{12}$ In addition, a BTS approach avoids extensive surgery in patients with disseminated disease or unacceptable surgical risk, since both SEMS and DC can also serve as a definitive palliative treatment. Both BTS approaches have their own (dis) advantages and no definite consensus exists on which treatment should be first choice.

A recent meta-analysis comparing SEMS placement with acute resection showed significantly lower morbidity, mortality and long-term stoma rates for patients who were treated with stent placement as BTS. ${ }^{13,14}$ However, SEMS as BTS has been under debate, especially in curative patients, since it has been suggested to negatively influence oncologic outcomes. ${ }^{15,18}$

A DC could prove a valid alternative to SEMS; colonic decompression is highly successful with this approach and patients are less likely to remain with a permanent stoma compared with acute resection. ${ }^{19-23}$ Furthermore, it is hypothesized that no long-term oncologic risks are associated with the construction of a DC. ${ }^{22}$ Deviating colostomy as BTS has, however, been abandoned in the past because of presumed prolonged hospital stay, increased number of surgical interventions and a possible negative influence on quality of life. Studies are, however, limited and of older date, and it may be questioned if this approach really is inferior to SEMS when performed according to the current standard of care.

Until now, no studies have directly compared colostomy construction with SEMS placement in curative patients. This retrospective multi-institutional cohort analysis aims to compare 
short- and long-term outcomes of SEMS placement vs. DC as BTS in patients presenting with acute colonic obstruction. According to the recent European Guidelines on Colonic Stent Placement, only patients with LSCO are studied. ${ }^{24}$

\section{METHODS}

\section{Study design and population}

A retrospective comparative study was conducted including all consecutive patients who presented with LSCO between January 2004 and December 2013 in two teaching hospitals in the Netherlands. Patients treated in Deventer Hospital (Hospital A) underwent SEMS placement as the initial treatment strategy, followed by elective resection. Patients in Meander Medical Center (Hospital B) underwent DC construction as first step.

Data were collected from the patients' medical records. Patients were included when they met the following inclusion criteria: 1 ) symptomatic malignant colonic obstruction (absence of stool, abdominal pain, nausea and/or vomiting) 2) left-sided location of the obstruction, defined as any tumor located in or distal to the splenic flexure and proximal to the rectum (with a minimum distance of $10 \mathrm{~cm}$ from the anal verge), 3) confirmation of colonic obstruction with abdominal X-ray or computed tomography (CT), 4) confirmation that the obstruction was caused by a histologically proven primary colon tumor and 5) a curative treatment intent as confirmed by the local multidisciplinary tumor board. Patients with resectable liver metastasis and patients eligible for HIPEC were also considered to have a curative perspective.

\section{Outcome parameters}

Demographic characteristics (age, gender and ASA-score) and medical history were collected from the medical records. In addition, the Charlson Co-morbidity Index (CCI) score was calculated in all patients. ${ }^{25}$ Furthermore, surgical characteristics such as TNM-stage, tumor differentiation, number of lymph nodes harvested, type of resection, type of stent and whether the patients were treated with adjuvant therapy were collected. Tumor staging was performed according to the American Joint Committee on Cancer Tumor Node Metastasis (TNM) classification, $5^{\text {th }}$ edition. ${ }^{26}$ The primary outcome measures were 30 -day morbidity and mortality. Complications were graded according to the classification of surgical complications developed and validated by Clavien and Dindo. ${ }^{27}$ Major morbidity was graded as III or higher, whereas minor morbidity was graded as II or lower. Results were collected for both the initial BTS approach as well as the subsequent elective resection.

Other study outcomes included technical and clinical success rates of stent placement, primary anastomosis rate, temporary colostomy rate, permanent colostomy rate, total hospital stay, disease recurrence and overall survival. Clinical success was defined as the production of feces within 48 hours after stent placement or colostomy construction. A BTS intent was considered to have failed when emergency surgery was still indicated. Permanent stoma rate was the number of patients who still had a colostomy during last follow-up with a minimum follow-up of at least 18 months. Hospital stay was the total number of days the patient was admitted including initial colonic decompression, elective resection and, if applicable, colostomy reversal. 
The initial bridge to surgery intervention: stent placement or colostomy construction All patients underwent the initial decompression procedure within 48 hours after presentation with obstructive symptoms. The gastro-intestinal surgeon on call performed the colostomy constructions, while SEMS placement was performed by one of three expert endoscopists with a minimum of 4 years experience ( $>20$ procedures) with colonic stent placement. Colostomies were constructed on the right transverse colon. An incision was made in the right upper abdominal quadrant; the transverse colon was elevated to the skin, temporarily anchored with a plastic rod and fixed with resolvable stitches. Details of the stent placing procedure have been published previously. ${ }^{28}$ Four different stent types were used, based on availability, i.e. Wallstent ${ }^{\circledast}$ (Boston Scientific, Natrick, MA, USA), Wallflex ${ }^{\circledast}$ (Boston Scientific), Ultraflex ${ }^{\circledR}$ (Boston Scientific) and Evolution ${ }^{\circledR}$ (Cook Medical, Limerick, Ireland).

After colostomy construction or stent placement, patients received enteral feeding as soon as possible. Elective resection was performed after approximately 2-4 weeks.

\section{Elective resection}

Experienced colorectal surgeons performed all elective resections, which were preferably performed laparoscopically with the construction of a primary anastomosis. In Hospital A, more open procedures were performed according to the preference of two surgeons. Based on the discretion of the treating surgeon, a protective deviating colostomy was created or, when the patient was treated with a colostomy as initial treatment, the existing colostomy was left in place. The resection included a routine mesocolic lymphadenectomy, with the goal of harvesting a minimum of 12 lymph nodes. The specific choice of resection was at the discretion of the treating surgeon and depended on tumor location, size and patient condition. Patients with resectable liver metastases underwent metastatectomy at a later stage, patients with resectable peritoneal metastases were referred for cytoreductive surgery and hyperthermic intraperitoneal chemotherapy at a tertiary center. Furthermore, patients with TNM-stage II (high risk patients only), III or IV were treated with adjuvant chemotherapy using either the FOLFOX (oxaliplatin, leucovorin and 5-fluoruoracil) or CAPOX (capecitabine, oxaliplatin) regime, if the patients' condition allowed it.

\section{Patient follow-up}

Patients were followed-up according to the Dutch guidelines. ${ }^{29}$ These guidelines include outpatient visits at a 6-month interval for the first 2-3 years and once a year until 5 years after the resection. A CT or abdominal ultrasound was made 6 months after resection and on a yearly basis thereafter. In addition, colonoscopy was performed approximately 2-3 years after resection. When there was suspicion of metastatic disease or recurrence, additional imaging was performed.

For the overall survival analysis, follow-up time was the number of months from presentation with LSCO until the last hospital visit, including visits to other physicians. For the disease-free survival analysis, follow-up time was the number of months from presentation with LSCO until the last evaluation at the surgical or oncological outpatient clinic. 


\section{Statistical analysis}

Statistical analyses were performed using the Statistical Program for the Social Sciences (SPSS) version 23.0 (SPSS ${ }^{\circledR}$ Inc., Chicago, IL). Continuous variables were tested for normality using the Kolmogorov-Smirnoff test, and when normally distributed, were reported as means with standard deviation. When continuous variables were non-normally distributed, they were reported as medians with interquartile range (i.q.r.). Continuous variables were compared between groups using an unpaired Student's t test or Mann-Whitney $U$ test depending on distribution. Categorical variables were described as counts and percentages and were compared using Fisher's exact test. Overall survival was analyzed by Kaplan-Meier statistics and treatment groups were compared using the log rank test. In addition, univariate and multivariate survival analyses were performed using Cox proportional-hazards regression methods to adjust for possible confounders. All $p$-values were two sided and $p<0.05$ was considered statistically significant. All analyses were performed on an intention-to-treat basis.

\section{RESULTS}

\section{Demographics and oncological characteristics}

Between 2004 and 2013, 325 patients presented at Hospital A and Hospital B with acute colonic obstruction. In total, 88 patients were eligible for inclusion because the obstruction was caused by colon carcinoma, located on the left side of the colon and they were initially considered treatable with curative intend. Fifty-one patients were treated with SEMS placement followed by elective resection while thirty-seven patients were treated with colostomy followed by elective resection (Figure 1). No significant differences were found in baseline characteristics, as is shown in Table 1.

\section{Surgical characteristics}

All patients eventually underwent an elective resection and no patients died after initial decompression. Colostomy construction resulted in successful colonic decompression and relief of symptoms within 24 hours in all 37 patients and no emergency resections were indicated. In addition, $59.5 \%(n=22)$ of the patients had immediate fecal discharge in the operating room when the stoma was opened. In almost all patients, a transversostomy was constructed $(n=34)$, three patients had a stoma constructed on the ascending or descending colon and one was treated with an ileostomy.

Four types of stents were placed, i.e. 17 Wallflex $^{\circledR}, 25$ Wallstent $^{\circledR}, 8$ Evolution $^{\circledR}$ and 1 Ultraflex ${ }^{\circledR}$ stents. Stent placement was technically successful in 49/51 (96.1\%) patients. Reasons for technical failure included perforation due to stent placement $(n=1)$ and inability to pass the guide-wire across the stricture $(n=1)$. Six patients with technically successful stent placement did not have any relief of symptoms, due to stent obstruction caused by fecal debris $(n=1)$, stent migration $(n=1)$ and unknown causes $(n=4)$. All these patients had to undergo an emergency resection. Therefore, stent placement was clinically successful in 43 (84.3\%) patients. Apart from the patients in whom SEMS placement was technically or clinically unsuccessful, relief of symptoms within 24 hours was seen in all patients. In addition, immediate fecal discharge following SEMS placement occurred in $58.8 \%$ of the patients $(n=30)$. 
FIGURE 1 | Study flowchart. A. Patients who underwent colostomy construction as initial treatment followed with elective surgery. B. Patients who underwent stent placement as BTS.
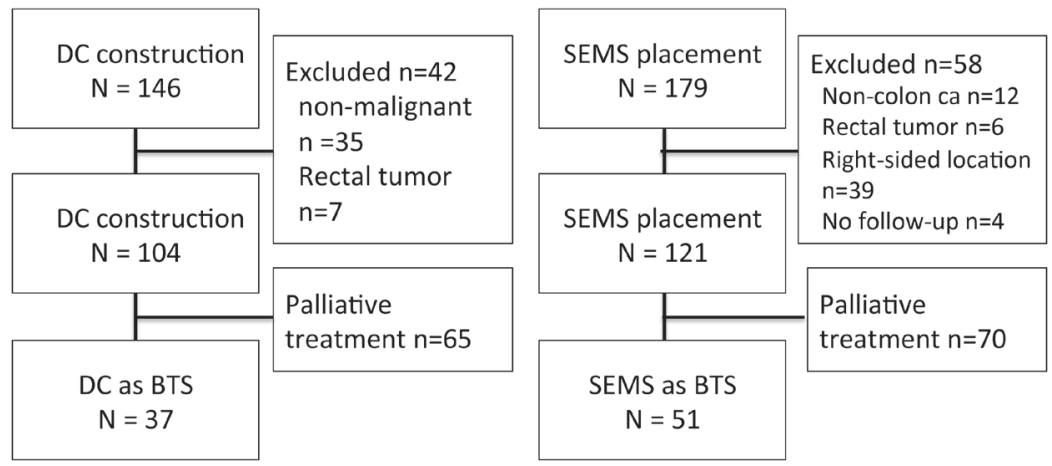

As shown in Table 1, no significant differences between treatment groups were found regarding resection type, the number of radical resection (RO) or the number of lymph nodes resected. More resections were performed laparoscopically in the DC group ( $35.1 \%$ vs. $15.7 \%, p<0.01)$, but this is most likely due to a difference in surgeons' preference between the two institutions, rather than the result of a different BTS approach used. Successful primary anastomosis construction was not different between both treatment groups, but the temporary stoma formation rate was significantly lower in the SEMS group ( $37.8 \%$ vs. $82.4 \%, p<0.01)$. Permanent stoma rates were not different between treatment groups ( $16.2 \%$ vs. $17.6 \%, p=0.86)$. (Table 2 )

\section{Outcome parameters}

\section{Morbidity and Mortality}

No significant difference in 30-day major and minor morbidity and mortality was observed between both treatment approaches (Table 2). Mortality occurred in one patient in each treatment group, and was caused by abdominal sepsis in both patients. A perforation occurred in one patient treated with SEMS, for which an emergency resection was performed; this resulted in anastomotic leakage and subsequent abdominal sepsis. The cause of abdominal sepsis in the DC patient was unclear.

Major morbidity in the SEMS group ( $n=13,25.5 \%)$ was mainly caused by stent dysfunction $(n=8)$. Four patients $(10.8 \%)$ in the DC group had a major complication, i.e. abdominal sepsis $(n=3)$ and wound dehiscence $(n=1)$, which was not found to be statistically different from the SEMS group ( $p=0.1$ ). In addition, after adjusting for potential confounders (age, gender, ASA score and surgical approach), SEMS placement was still not associated with a higher 30-day mortality risk (Hazard Rate (HR) $1.12 \quad(95 \% \mathrm{Cl} 0.94-1.33)$ ). A detailed overview of all complications is shown in Table 3.

The long-term complications rate was significantly more favorable in the SEMS group compared to the DC group ( $29.7 \%$ vs. $9.8 \%$, respectively, $p=0.03$ ). This difference was mainly caused by a higher percentage of incisional hernias in the DC group compared to the SEMS group; $18.9 \%$ 
TABLE 1 | Demographic, oncologic and surgical characteristics

\begin{tabular}{|c|c|c|c|}
\hline & $\begin{array}{l}\text { Colostomy group } n(\%) \\
\qquad N=37\end{array}$ & $\begin{array}{l}\text { SEMS group } n(\%) \\
\quad \mathrm{N}=\mathbf{5 1}\end{array}$ & p-value \\
\hline Age in yearsๆ & $66.63(13.2)$ & $71.8(13.1)$ & $0.07 \#$ \\
\hline Gender male:female & $14: 23$ & $25: 26$ & 0.31 \\
\hline Age adjusted $\mathrm{CCl}$-scoreף & $4.5(1.8)$ & $5.2(1.7)$ & $0.09 \#$ \\
\hline ASA score & & & 0.10 \\
\hline 1 & $20(54.1)$ & $17(33.3)$ & \\
\hline II & $16(43.2)$ & $29(56.9)$ & \\
\hline III & $1(2.7)$ & $5(9.8)$ & \\
\hline IV & $0(0)$ & $0(0)$ & \\
\hline pTNM stage & & & 0.48 \\
\hline 1 & $0(0)$ & $3(5.9)$ & \\
\hline II & $17(45.9)$ & $23(45.1)$ & \\
\hline III & $16(43.2)$ & $20(39.2)$ & \\
\hline IV & $4(10.8)$ & $4(7.8)$ & \\
\hline Tumor differentiation & & & 0.23 \\
\hline Well & $0(0)$ & $2(3.9)$ & \\
\hline Moderate & $33(89.2)$ & $46(90.2)$ & \\
\hline Poor & $4(10.8)$ & $2(3.9)$ & \\
\hline Unknown & $0(0)$ & $1(1.9)$ & \\
\hline Tumor Location & & & 0.92 \\
\hline Sigmoid & $27(73.0)$ & $39(76.5)$ & \\
\hline Descending colon & $7(21.6)$ & $8(15.7)$ & \\
\hline Splenic flexure & $3(8.1)$ & $4(7.8)$ & \\
\hline Resection & & & 0.33 \\
\hline RO & $37(100)$ & $48(94.1)$ & \\
\hline $\mathrm{R} 1$ & 0 & 0 & \\
\hline Unknown & 0 & $2(3.9)$ & \\
\hline Number of lymph nodes harvested§ & $14.5(10.5-19.8)$ & $13(10.5-18.5)$ & $0.71 \mathrm{C}$ \\
\hline Follow-up in months last check-up§ & $29(20.5-62)$ & $36(17-59)$ & $0.91 c$ \\
\hline Follow-up in months alive $\S$ & $30(21-67)$ & $46(26-82)$ & $0.24 \mathrm{C}$ \\
\hline Resection type & & & 0.21 \\
\hline Left hemicolectomy & $10(27.0)$ & $16(31.4)$ & \\
\hline Sigmoidectomy & $17(35.9)$ & $22(43.1)$ & \\
\hline Low anterior resection & $6(16.2)$ & $4(7.8)$ & \\
\hline Subtotal colectomy & $2(5.4)$ & $1(1.9)$ & \\
\hline Hartmann & $1(2.7)$ & $8(15.7)$ & \\
\hline Type of procedure & & & $<0.01^{\mathrm{a}}$ \\
\hline Laparotomy & $19(51.4)$ & $43(84.3)$ & \\
\hline Laparoscopy & $13(35.1)$ & $8(15.7)$ & \\
\hline Conversion & $5(21.7)$ & $2(25)$ & \\
\hline Days to resection§ & $25(16.5-35)$ & $17(10-24)$ & $<0.01 C$ \\
\hline Patients who received adjuvant chemotherapy & $20(54.1)$ & $17(19.6)$ & 0.05 \\
\hline TNM stage I & $0(0)$ & $0(0)$ & \\
\hline TNM stage II & $5(29.4)$ & $0(0)$ & $<0.01$ \\
\hline TNM stage III & $12(75)$ & $14(70)$ & 0.74 \\
\hline TNM stage IV & $3(75)$ & $3(75)$ & 0.78 \\
\hline
\end{tabular}

Values are ๆ means (SD) and § median (inter quartile range). All comparisons were made using Fisher's exact test, except: \#one-way ANOVA and $\mathrm{C}=$ Mann-Withney $\mathrm{U}$ test. ${ }^{\mathrm{a}}$ This difference is most likely due to a difference in surgeons' preference between the two institutions, rather than the result of a different BTS approach. Abbreviations :CAPOX= capecitabine and oxaliplatin, FOLFOX = oxaliplatin, leucovorin and 5-fluorouracil). 
TABLE 2 | Overview of all study outcomes

\begin{tabular}{|c|c|c|c|}
\hline & $\begin{array}{l}\text { Colostomy group } \mathrm{n}(\%) \\
\mathrm{N}=\mathbf{3 7}\end{array}$ & $\begin{array}{l}\text { SEMS group } n(\%) \\
\qquad N=51\end{array}$ & p-value \\
\hline 30-day mortality & $1(2.7)$ & $1(1.9)$ & 0.82 \\
\hline Complications overall & $12(32.4)$ & $23(45.1)$ & 0.23 \\
\hline \multicolumn{4}{|l|}{ 30-day morbidity } \\
\hline Major morbidity & $4(10.8)$ & $13(25.4)$ & 0.10 \\
\hline Minor morbidity & $11(29.7)$ & $12(23.5)$ & 0.67 \\
\hline Hospital stay in days§ & $14(11-17)$ & $14(11-17)$ & $0.78 c$ \\
\hline Immediate feacal production & $22(59.5)$ & $58.8(30)$ & 0.95 \\
\hline Relief of symptoms $<24$ hours & $37(100.0)$ & $45(88.2)$ & 0.04 \\
\hline Number of interventions§ (i.q.r.) & $3.0(2-4)$ & $2.0(2-4)$ & $<0.01 \mathrm{C}$ \\
\hline $\begin{array}{l}\text { Primary anastomosis without } \\
\text { colostomy }\end{array}$ & $14(37.8)$ & $42(82.4)$ & $<0.01$ \\
\hline Permanent colostomy & $6(16.2)$ & $9(17.6)$ & 0.86 \\
\hline Long term complications & $11(29.7)$ & $5(9.8)$ & 0.01 \\
\hline Local recurrence in follow-up & $4(10.8)$ & $2(3.9)$ & 0.21 \\
\hline Metastasis in follow-up & 7 (18.9) & $7(13.7)$ & 0.51 \\
\hline 3-year survival & & & 0.83 \\
\hline Alive & $17 / 22(77.3)$ & $35 / 44(79.5)$ & \\
\hline Unknown & 15 & 6 & \\
\hline 5-year survival & & & 0.43 \\
\hline Alive & $14 / 19$ (73.7) & $24 / 38(63.2)$ & \\
\hline Unknown & 18 & 13 & \\
\hline
\end{tabular}

Values are Imeans (SD) and §median (inter quartile range). All comparisons were made using Fisher's exact test, except: \#one-way ANOVA and CMann-Withney $U$ test. Number of surgeries: includes the BTS procedure, the elective resection, surgeries performed for complications and possible surgeries for stoma reversal; surgeries for long-term complications were not included.

(7/37) vs. 9.8\% (5/51), respectively. In addition, five of seven patients in the DC group underwent surgical repair of the hernia, whereas surgical intervention was only indicated in one of five patients in the SEMS group (Table 3).

\section{Hospital stay and the number of interventions}

Median (i.q.r) hospital stay was 14 (11-17) days in both treatment groups ( $p=0.78)$. Patients treated with DC had a median number of $3(2-4)$ surgical interventions per patient, which was significantly higher than the number of interventions performed in the SEMS group (2 (2-4), $\mathrm{p}<0.01)$.

\section{Disease recurrence and overall survival}

Median (i.q.r.) follow-up was 29 (20.5-62) months in the DC group and 36 (17-59) months in the SEMS group $(p=0.91)$. No significant differences were found in possible confounders of long-term oncological outcomes; e.g. age, ASA-score, age adjusted $\mathrm{CCl}$-score, tumor stage, number of radical resections and number of lymph nodes resected. The use of adjuvant chemotherapy differed between the two hospitals. When treatment with adjuvant chemotherapy was analyzed according to tumor stage, significantly more stage II patients were 
TABLE 3 | Overview of all complications during total follow-up

\begin{tabular}{|c|c|c|}
\hline & $\begin{array}{c}\text { Colostomy group } \\
\qquad N=37\end{array}$ & $\begin{array}{l}\text { SEMS group } \\
\qquad N=51\end{array}$ \\
\hline \multicolumn{3}{|l|}{ Major morbidity } \\
\hline Stent perforation & - & 1 \\
\hline Stent dysfunction & - & 7 \\
\hline Technical placement failure & - & 2 \\
\hline Clinical stent failure (unknown cause) & - & 4 \\
\hline Overgrowth & - & - \\
\hline Feacal impaction & - & 1 \\
\hline Stent migration & - & 1 \\
\hline Anastomotic leakage & - & $1\{1\}$ \\
\hline Abdominal sepsis & $3\{1\}$ & - \\
\hline Wound dehiscence & 1 & 2 \\
\hline Other* & 0 & 2 \\
\hline \multicolumn{3}{|l|}{ Minor Morbidity } \\
\hline Wound infection & 4 & 1 \\
\hline Pneumonia & 4 & 5 \\
\hline Urinal tract infection & 1 & 4 \\
\hline Urine retention & 0 & 2 \\
\hline Other** & 2 & 2 \\
\hline \multicolumn{3}{|l|}{ Long term complications } \\
\hline Cicatricial hernia & 7 & 5 \\
\hline Enterocutaneous fistula & 1 & 0 \\
\hline Abscess abdominal wall & 1 & 0 \\
\hline Stenosis anastomosis & 1 & 0 \\
\hline
\end{tabular}

*Other includes: splenic rupture during surgery (1), cystic damage during surgery (1). Abdominal sepsis causes were: wound infection (2) and unknown (1). **Other includes: cerebral hemorrhage (1), new atrial fibrillation (10) deliria (1) and minor bleed (1). The numbers in \{brackets\} represent patients that died within 30-days after initial decompression or elective resection.

treated with adjuvant chemotherapy in the DC group ( $25.0 \%$ vs. $0 \%, p=0.005)$. We therefore stratified patients for tumor stage and analyzed them separately for stage I\&II and stage III\&IV (Figure 2). No significant differences in disease free survival were found between the treatment groups when stratified for tumor stage (stage I\&II log rank 1.7, P=0.19, stage III\&IV: log rank=1.2, $\mathrm{P}=0.27$.). Median survival was not reached in any TNM-stage. In addition, no significant differences in overall survival between treatment groups was found; stage I\&II: log rank 1.9, $\mathrm{P}=0.17$, stage III\&IV: log rank $0.55, \mathrm{P}=0.46$.

As no significant difference in survival was found when data was stratified by tumor stage, pooled disease recurrence and overall survival were also analyzed (Figure 3 ). These analyses also did not show significant differences in disease free survival (log rank=0.03, $\mathrm{P}=0.96)$ and overall survival (log rank=0.25, $\mathrm{P}=0.61$ ). In addition, adjustment for possible confounders (age (<70 vs. $\geq 70$ ), ASA-score (II-II vs. III-IV), TNM-stage (I-II vs. III-IV) and adjuvant therapy (yes/ no)) using a multivariable Cox model also did not result in a statistically significant association between SEMS and death (Hazard ratio HR $0.98(95 \% \mathrm{Cl} 0.37-2.57), \mathrm{p}=0.96)$ or SEMS and disease recurrence ( $\mathrm{HR} 0.84(95 \% \mathrm{Cl} 0.3-2.3), \mathrm{p}=0.73)$. 
FIGURE 2 | Survival analysis using Kaplan-Meier analyses stratified by tumor stage. A. Overall survival in stage I\&II, logrank=1.9, $P=0.17$. B. Overall survival in stage III\&IV, logrank $=0.55, P=0.46 \mathrm{C}$. Disease free survival in TNM-stage I\&II, $\operatorname{logrank}=1.7, P=0.19 \mathrm{D}$. Disease free survival in stage III\&IV, logrank=1.2, $\mathrm{P}=0.27$ ).

A

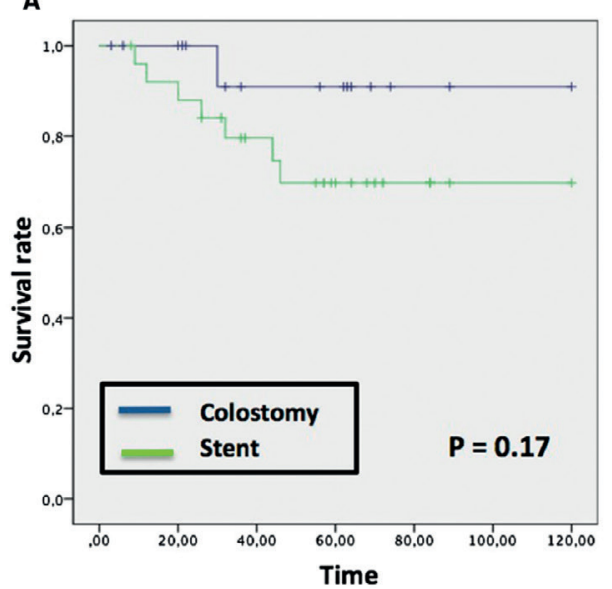

C

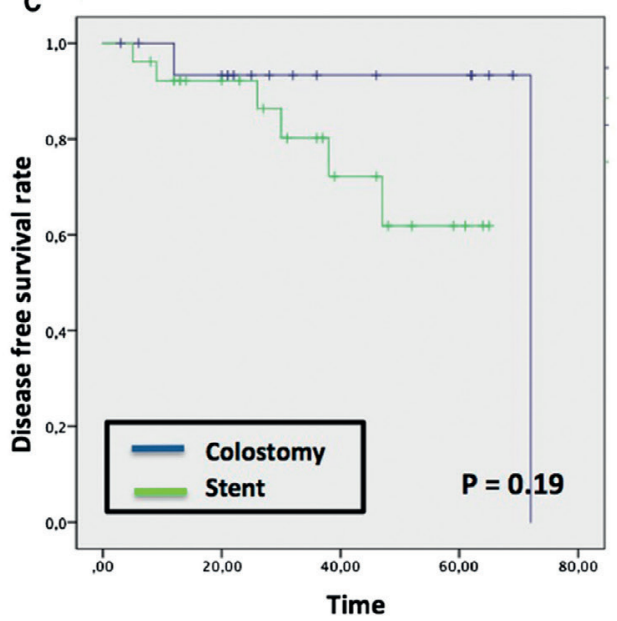

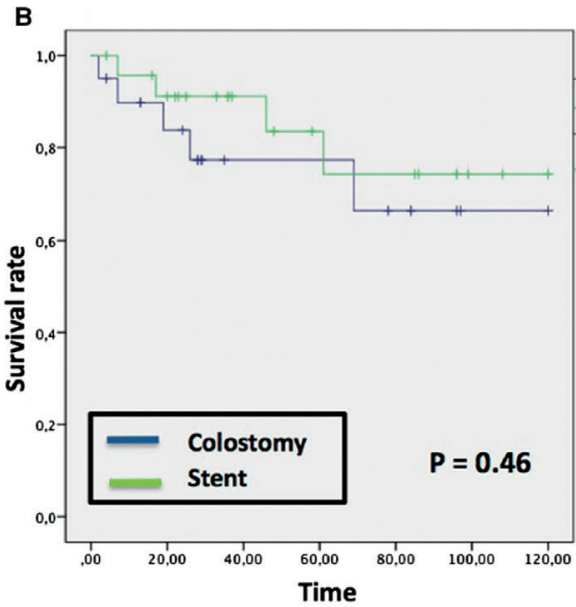

D

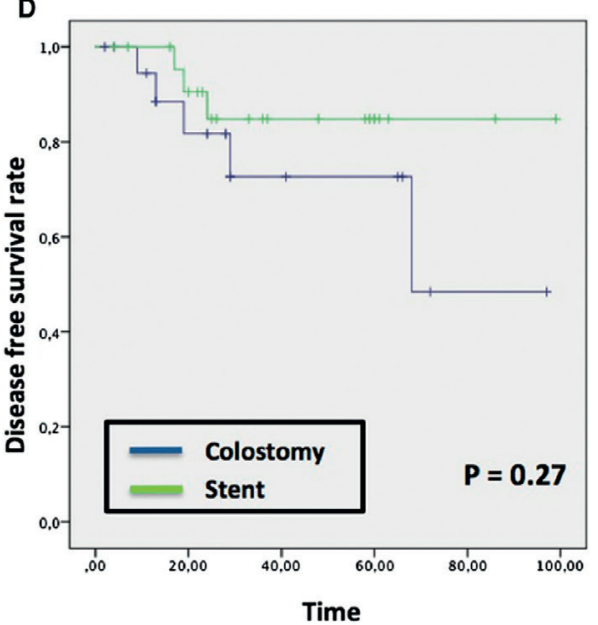

\section{DISCUSSION}

Hospital stay, morbidity rates, mortality rates, recurrence rates and overall survival were not significantly different between both treatment groups. The number of temporary stomas, surgical interventions and long-term complications were, however, significantly lower in the SEMS group. According to a recent Dutch population based analysis, approximately $81.8 \%$ of all patients with acute LSCO are treated with acute resection in the Netherlands, $10.8 \%$ with stent placement and $7.4 \%$ with a colostomy as BTS. ${ }^{9}$ These numbers seem to indicate that a BTS approach is not yet a routine procedure in the current treatment practice for patients. 
FIGURE 3 | Survival analysis using Kaplan-Meier analyses without stratification. A. Overall survival, logrank=0.25, P=0.61. B. Disease free survival, logrank=0.03, $P=0.96$.
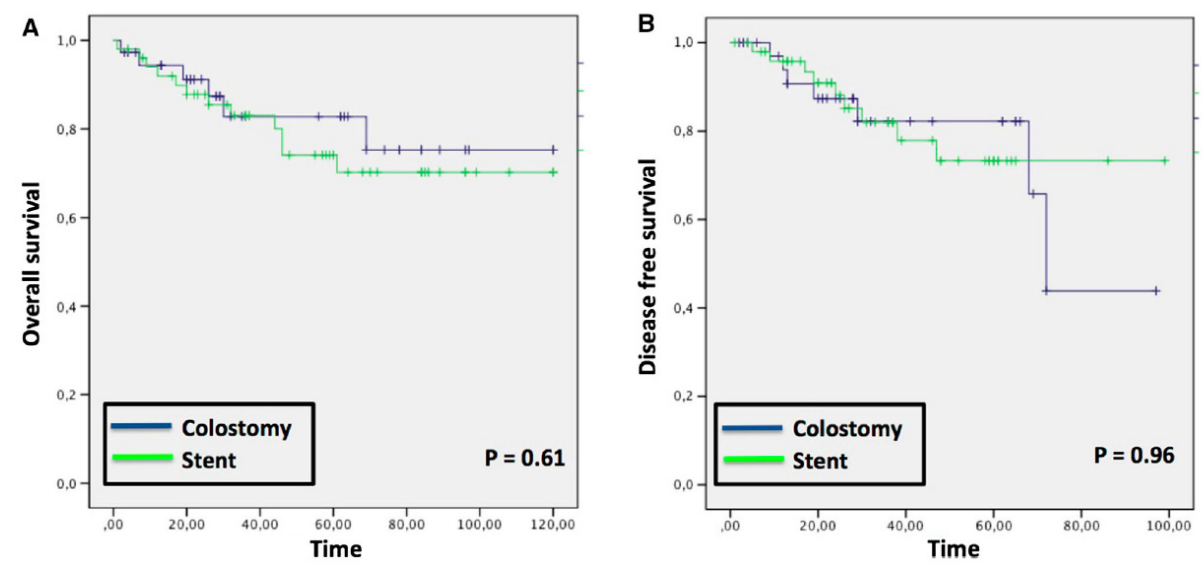

The only available study comparing SEMS with DC as BTS in curative patients is a recent population-based analysis by Tanis et al. (2015). In accordance to our study, they found no significant difference in morbidity rates, hospital stay, number of radical resections, number of lymph nodes retrieved and mortality rates. However, this study is limited by the fact that only patients that eventually underwent an elective resection were included, while those not undergoing a resection, for instance due to premature death or other problems, were not included. This could have positively biased the results. ${ }^{9}$ These patients were not excluded from the analyses in our study. Furthermore, two randomized studies comparing SEMS with DC in the palliative setting were identified. These authors also found no difference in morbidity and mortality rates following initial decompression. ${ }^{34,35}$ However, since SEMS placement or DC construction was performed as a definitive treatment and patients did not undergo subsequent resection, other outcomes were difficult to compare with those in the current study.

Presumed prolonged hospital stay, an increased number of surgical interventions and a negative influence on quality of life (QoL) have been reasons to abandon DC as BTS in the past. ${ }^{31}$ The current study confirms a higher surgical intervention rate in the DC group. Colostomy reversal during a third surgical procedure and incisional hernias repair are the main reasons why the number of surgical procedures is increased in the DC group. Indeed, incisional hernia rates of up to $30 \%$ following stoma closure have been reported in prospective studies and approximately $50 \%$ of these patients eventually require surgical correction. ${ }^{32}$ In the current study a $19 \%$ incisional hernia rate was found in the DC group and surgical correction was performed in $71.4 \%$ of these patients, compared to $9 \%$ and $20 \%$ in the SEMS group, respectively $(p=0.35$ and $p<0.01)$. Interestingly, the fact that patients treated with DC underwent significantly more surgical interventions did not result in a prolonged hospital stay. Another presumed disadvantage of DC as BTS is that it is thought to decrease QoL. Indeed, a recent randomized 
controlled trial by Young et al. (2015) showed a significant and persistent better QoL in palliative patients treated with SEMS versus emergency surgery (either colostomy or acute resection). ${ }^{10}$ No data on QoL was collected in the present study, but it is unlikely that QoL was severely affected in the DC group, as $>80 \%$ of the DC patients had their colostomy reversed and DC was not intended as a definite palliative measure. All currently available studies reporting on a decreased QoL in stoma patients include only patients with a permanent stoma and it remains to be determined whether $\mathrm{QoL}$ is also decreased in patients that have their stoma reversed. ${ }^{10,11,20}$

SEMS placement was highly successful in the current study (clinical success $84.3 \%$ ) and only $17 \%$ of the patients had a stoma constructed. These findings are in line with findings of metaanalyses and systematic reviews, reporting on short-term success and stoma rates of around $90 \%$ and $15 \%$, respectively. ${ }^{33,36,37}$ However, since two randomized trials investigating SEMS placement have been closed prematurely due to SEMS-related complications, it is important to also focus on specific complications caused by stent placement. ${ }^{38,39}$ Indeed, this study underlines that the consequences of SEMS-related perforations could be severe; SEMS-related perforation is solely accountable for mortality in the SEMS group in our study. To keep perforation rate following SEMS placement as small as possible, stents should not be placed in patients with an increased risk of stent-related colonic perforation. Currently known risk factors for stent-related perforation are; benign obstruction, post-stent dilatation and treatment with bevacizumab (or any other angiogenesis-inhibitor)..$^{40} \mathrm{DC}$ construction should therefore always be the preferred BTS approach in patients with one or more of these risk factors. Stent re-obstruction and migration rates are relatively low in the current study, which is probably due to the fact that an elective resection was generally performed within 4 weeks and these complications often occur when stents are longer in place..$^{33,36,40,41}$ In addition, uncertainty about the effect of SEMS on long-term oncological outcomes makes many physicians hesitant to use this approach in curative patients. ${ }^{15-18}$

Studies comparing SEMS and DC on long-term oncologic outcomes have not been performed up until now. The oncologic safety of SEMS has been under debate for several years, whereas colostomy construction is hypothesized not to be associated with an increased oncologic risk. ${ }^{22}$ The present study found no significant difference in disease free survival as well as overall survival between both treatment groups. Even though results are based on a limited number of patients, this study did not substantiate the fear of negative oncological impact after SEMS placement due to (micro) perforations. Several other studies comparing stent placement with acute resection are in line with these results. ${ }^{41,42}$ However, there are studies that did find significantly worse oncologic outcomes for patients treated with SEMS placement as BTS versus acute resection. ${ }^{17,43-45}$ This could be explained by the fact that a relatively high number of stent-related perforations occurred in these studies, which is associated with significantly worse oncologic outcomes. ${ }^{17}$ As stated above, one (1.9\%) stent-related perforation occurred in the present study and this patient unfortunately died due to abdominal sepsis after emergency surgery and was therefore no longer at risk for disease recurrence. 
This study is limited by its retrospective design. Possible bias could also have been introduced by the two-center design of the study. This restriction was minimalized since, except for the BTS approach, perioperative management was similar in both hospitals. Both are large teaching hospitals in the Netherlands with a similar volume of colorectal operations. In addition, although baseline characteristics were not significantly different, multivariate regression analyses were conducted when possible in order to minimize the risk of possible confounders influencing the results.

Another limitation of this study was the small number of patients, which was due to our strict in- and exclusion criteria. As more data are required on the role of a bridge to surgery approach, further studies are warranted. We are currently participating in a Dutch prospective multicenter trial that compares the different treatment options as suggested by the recently introduced European Guidelines on the treatment of left-sided colonic obstructions. ${ }^{24}$

In conclusion, SEMS placement and DC construction are both effective decompression methods for acute LSCO in patients who may be cured by resection. SEMS placement seems to result in fewer surgical interventions and long-term complications. However, the consequences of SEMS-related perforations could be severe and safety with regard to the oncologic outcomes can as yet not be guaranteed. Long-term oncological data from prospective trials are therefore awaited before stent placement can broadly be applied in patients with acute LSCO. 


\section{REFERENCES}

1. Jullumstro E, Wibe A, Lydersen S, Edna TH. Colon cancer incidence, presentation, treatment and outcomes over 25 years. Colorectal Dis, 2011;13(5):512-8

2. Cheynel N, Cortet M, Lepage C, Benoit L, Faivre J, Bouvier AM. Trends in frequency and management of obstructing colorectal cancers in a well-defined population. Dis Colon Rectum 2007;50:1568-75

3. Scott NA, Jeacock J, Kingston RD. Risk factors in patients presenting as an emergency with colorectal cancer. $\mathrm{Br}$ J Surg 1995182(3):321-3

4. Hennessey DB, Burke JP, Ni-Dhonochu T, Shields C, Winter DC, Mealy K. Preoperative hypoalbuminemia is an independent risk factor for the development of surgical site infection following gastrointestinal surgery: a multi-institutional study. Ann Surg 2010;252(2):325-9

5. Sjo OH, Larsen S, Lunde OC, Nesbakken A. Short term outcome after emergency and elective surgery for colon cancer. Colorectal Dis 2009;11:733-739

6. Papadimitriou G1, Manganas D, Phedias Georgiades C, Vougas V, Vardas K, Drakopoulos S. Emergency surgery for obstructing colorectal malignancy: prognostic and risk factors. J BUON 2015;20(2):406-12

7. Tan KK, Sim R. Surgery for obstructed colorectal malignancy in an Asian population: predictors of morbidity and comparison between left- and right-sided cancers. J Gastrointest Surg 2010;14:295

8. Iversen LH, Bülow S, Christensen IJ, Laurberg S, Harling $\mathrm{H}$; Danish. Colorectal Cancer Group. Postoperative medical complications are the main cause of early death after emergency surgery for colonic cancer. Br J Surg 2008;95:1012-9

9. Tanis PJ, Paulino Pereira NR, van Hooft JE, Consten EC, Bemelman WA. Resection of Obstructive Left-Sided Colon Cancer at a National Level: A Prospective Analysis of Short-Term Outcomes in 1,816 Patients. Dig Surg 2015;4;32(5):317-324

10. Young CJ, De-Loyde KJ, Young JM, Solomon MJ, Chew EH, Byrne CM, Salkeld G, Faragher IG Improving Quality of Life for People with Incurable Large-Bowel Obstruction: Randomized Control Trial of Colonic Stent Insertion. Dis Colon Rectum 2015;58(9):838-49

11. Anaraki F, Vafaie M, Behboo R, Maghsoodi N, Esmaeilpour S, Safaee A. Quality of life outcomes in patients living with stoma. Indian J Palliat Care 2012;18(3):176-80

12. Bosscher MR, van Leeuwen BL, Hoekstra HJ. Current management of surgical oncologic emergencies. PLoS One 2015;10(5):e0124641
13. Huang X, Lv B, Zhang S, Meng L. Preoperative colonic stents versus emergency surgery for acute left-sided malignant colonic obstruction: a meta-analysis. J Gastrointest Surg 2014;18(3):584-91

14. Zhao XD, Cai BB, Cao RS, Shi RH. Palliative treatment for incurable malignant colorectal obstructions: a meta-analysis. World J Gastroenterol. 2013;7; 19(33):5565-74

15. Maruthachalam K, Lash GE, Shenton BK, Horgan AF. Tumour cell dissemination following endoscopic stent insertion. Br J Surg 2007;94:1151-1154

16. Erichsen R, Horváth-Puhó $\mathrm{E}$, Jacobsen JB, Nilsson $\mathrm{T}$, Baron JA, Sørensen HT. Long-term mortality and recurrence after colorectal cancer surgery with preoperative stenting: a Danish nationwide cohort study. Endoscopy 2015;47(6):517-24

17. Sloothaak DA, van den Berg MW, Dijkgraaf MG, Fockens P, Tanis PJ, van Hooft JE, Bemelman WA collaborative Dutch Stent-In study group. Oncological outcome of malignant colonic obstruction in the Dutch Stent-In 2 trial. Br J Surg. 2014;101(13):1751-7

18. Sabbagh C, Browet F, Diouf M, Cosse C, Brehant O, Bartoli E, Mauvais F, Chauffert B, Dupas JL, Nguyen-Khac E, Regimbeau JM. Is Stenting as "a Bridge to Surgery" an Oncologically Safe Strategy for the Management of Acute, Left-Sided, Malignant, Colonic Obstruction?: A Comparative Study With a Propensity Score Analysis. Ann Surg 2013;258:107-115

19. Park JJ, Del Pino A, Orsay CP, Nelson RL, Pearl RK, Cintron JR, Abcarian H. Stoma complications: the Cook County Hospital experience. Dis Colon Rectum 1999; 42: $1575-80$

20. Sprangers MA, Taal BG, Aaronson NK, te Velde A. Quality of life in colorectal cancer. Stoma vs. nonstoma patients. Dis Colon Rectum 1995;38(4):361-9

21. Gandrup P, Lund L, Balslev I. Surgical treatment of acute malignant large bowel obstruction. Eur J Surg 1992;158: 427-430

22. Jiang JK, Lan YT, Lin TC, Chen WS, Yang SH, Wang HS, Chang SC, Lin JK. Primary vs. delayed resection for obstructive left-sided colorectal cancer: impact of surgery on patient outcome. Dis Colon Rectum 2008;51(3):306-11

23. Kronborg O. Acute obstruction from tumour in the left colon without spread. A randomized trial of emergency colostomy versus resection. Int J Colorectal Dis 1995;10(1):1-5. 
24. van Hooft JE, van Halsema EE, Vanbiervliet $\mathrm{G}$, Beets-Tan RG, DeWitt JM, Donnellan F, Dumonceau JM, GlynneJones RG, Hassan C, Jiménez-Perez J, Meisner S, Muthusamy VR, Parker MC, Regimbeau JM, Sabbagh C, Sagar J, Tanis PJ, Vandervoort J, Webster GJ, Manes G, Barthet MA, Repici A. Self-expandable metal stents for obstructing colonic and extracolonic cancer: European Society of Gastrointestinal Endoscopy (ESGE) Clinical Guideline. Gastrointest Endosc 2014;80(5):747-61.e1-75

25. Charlson ME, Pompei P, Ales KL, MacKenzie CR. A new method of classifying prognostic comorbidity in studies: development and validation. J Chronic Dis 1987;40:373383

26. Sobin LH, Fleming ID. TNM Classification of Malignant Tumors, fifth edition (1997). Union Internationale Contre le Cancer and the American Joint Committee on Cancer. Cancer 1997;80: 1803-1804

27. Dindo D, Demartines N, Clavien PA. Classification of surgical complications: a new proposal with evaluation in a cohort of 6336 patients and results of a survey. Ann Surg 2004;240: 205-213

28. Otchy D, Hyman NH, Simmang C, Anthony T, Buie WD, Cataldo P, Church J, Cohen J, Dentsman F, Ellis CN, Kilkenny JW 3rd, Ko C, Moore R, Orsay C, Place R, Rafferty J, Rakinic J, Savoca P, Tjandra J, Whiteford M. Practice parameters for colon cancer. Dis Colon Rectum 2004;47(8):1269-84

29. Integraal Kankercentrum Nederland. National Working Group on Gastrointestinal Cancers. Guideline Colon Cancer 2.0. Available at: http://www.oncoline.nl/ coloncarcinoom. Accessed 1 June 2015

30. Tan CJ, Dasari BV, Gardiner K. Systematic review and meta-analysis of randomized clinical trials of selfexpanding metallic stents as a bridge to surgery versus emergency surgery for malignant left-sided large bowel obstruction. Br J Surg 2012;99: 469-476.

31. Amelung FJ, Mulder CLJ, Verheijen PM, Draaisma WA, Siersema PD, Consten ECJ. Acute resection versus bridge to surgery with diverting colostomy for patients with acute malignant left-sided colonic obstruction: systematic review and meta-analysis. Surgical Oncology $2015 ; 24 ; 313-321$

32. Bhangu A, Nepogodiev D, Futaba K; West Midlands Research Collaborative. Systematic review and metaanalysis of the incidence of incisional hernia at the site of stoma closure. World J Surg. 2012;36(5):973-83

33. Liang TW, Sun Y, Wei YC, Yang DX. Palliative treatment of malignant colorectal obstruction caused by advanced malignancy: a self-expanding metallic stent or surgery? a system review and meta-analysis. Surg Today 2014;44:22-33
34. Fiori E, Lamazza A, De Cesare A, Bononi M, Volpino P, Schillaci A, Cavallaro A, Cangemi V. Palliative management of malignant rectosigmoidal obstruction. Colostomy vs. endoscopic stenting. A randomized prospective trial. Anticancer Res. 2004;24(1):265-8

35. Xinopoulos D, Dimitroulopoulos $D$, Theodosopoulos $T$, Tsamakidis K, Bitsakou G, Plataniotis G, Gontikakis M, Kontis M, Paraskevas I, Vassilobpoulos P, Paraskevas E. Stenting or stoma creation for patients with inoperable malignant colonic obstructions? Results of a study and cost-effectiveness analysis. Surg Endosc 2004;18(3):421-6

36. Sebastian $\mathrm{S}$, Johnston $\mathrm{S}$, Geoghegan $\mathrm{T}$, Torreggiani $\mathrm{W}$ Buckley M. Pooled analysis of the efficacy and safety of selfexpanding metal stenting in malignant colorectal obstruction. Am J Gastroenterol 2004;99:2051-2057

37. Zhao XD, Cai BB, Cao RS, Shi RH. Palliative treatment for incurable malignant colorectal obstructions: a metaanalysis. World J Gastroenterol 2013;19:5565-5574

38. van Hooft JE, Bemelman WA, Oldenburg B, Marinelli AW, Lutke Holzik MF, Grubben MJ, Sprangers MA, Dijkgraaf MG, Fockens P. Colonic stenting versus emergency surgery for acute left-sided malignant colonic obstruction: a multicentre randomised trial. Lancet Oncol 2011;12: 344-352

39. Pirlet IA, Slim K, Kwiatkowski F, Michot F, Millat BL. Emergency preoperative stenting versus surgery for acute left-sided malignant colonic obstruction: a multicenter randomized controlled trial. Surg Endosc 2011;25(6):1814-21

40. van Halsema EE, van Hooft JE, Small AJ, Baron TH, García-Cano J, Cheon JH, Lee MS, Kwon SH, MucciHennekinne S, Fockens P, Dijkgraaf MG, Repici A. Perforation in colorectal stenting: a meta-analysis and a search for risk factors. Gastrointest Endosc. 2014;79(6):970-82.e7

41. Abbott S, Eglinton TW, Ma Y, Stevenson C, Robertson GM, Frizelle FA. Predictors of outcome in palliative colonic stent placement for malignant obstruction. $\mathrm{Br}$ J Surg 2014;101:121-126

42. Small AJ, Coelho-Prabhu N, Baron TH. Endoscopic placement of self-expandable metal stents for malignant colonic obstruction: long-term outcomes and complication factors. Gastrointest Endosc 2010;71:560572

43. Kim HJ, Huh JW, Kang WS, Kim CH, Lim SW, Joo YE, Kim HR, Kim YJ. Oncologic safety of stent as bridge to surgery compared to emergency radical surgery for leftsided colorectal cancer obstruction. Surg Endosc 2013;27(9):3121-8 
44. Choi JM, Lee C, Han YM, Lee $M$, Choi YH, Jang DK, Im JP, Kim SG, Kim JS, Jung HC. Long-term oncologic outcomes of endoscopic stenting as a bridge to surgery for malignant colonic obstruction: comparison with emergency surgery. Surg Endosc 2014;28(9):2649-55

45. Gorissen KJ, Tuynman JB, Fryer E, Wang L, Uberoi R, Jones OM, Cunningham C, Lindsey I Local recurrence after stenting for obstructing left-sided colonic cancer. Br J Surg 2013;100: 1805-1809
46. Kim JS, Hur H, Min BS, Sohn SK, Cho CH, Kim NK. Oncologic outcomes of self-expanding metallic stent insertion as a bridge to surgery in the management of left-sided colon cancer obstruction: comparison with nonobstructing elective surgery. World J Surg; 2009;33: 1281-1286

47. Sabbagh C, Browet F, Diouf M, Cosse C, Brehant O, Bartoli $E$ et al. Is stenting as 'a bridge to surgery' an oncologically safe strategy for the management of acute, left-sided, malignant, colonic obstruction?: a comparative study with a propensity score analysis. Ann Surg; 2013;258: 107-115 



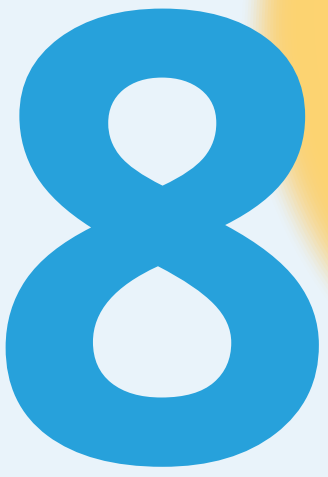

Ileostomy versus colostomy: which is preferable? Translated from Dutch

Nederlands Tijdschrift voor Geneeskunde 2017
F.J. Amelung
C.P. van t Hullenaar
P.M. Verheijen
E.C.J. Consten 


\section{ABSTRACT}

\section{Background}

To investigate whether an ileostomy or colostomy should be preferred in patients requiring temporary deviating stoma construction.

\section{Method}

A systematic literature search and retrospective comparative analysis was conducted. PubMed, Embase and Cochrane Library were searched for literature comparing loop ileostomy and loop colostomy. In addition, we retrospectively analyzed 361 patients who received a temporary loop ileostomy or loop colostomy between 2009 and 2015 in our hospital, Meander Medical Center in the Netherlands. Outcome measures were: quality of life, short-term and long-term complications.

\section{Results}

Literature search yielded 2092 studies, of which eight were included following full text screening. Six of these studies recommended ileostomy construction over colostomy construction; no preference was given in the other two studies. In addition, we analyzed 361 patients from our own hospital that had a temporary deviating stoma constructed. $49.6 \%$ (n $=179)$ had a colostomy constructed, the other $50.4 \%(n=182)$ an ileostomy. Significantly fewer stoma retractions $(p<0.01)$ and incisional hernias $(p<0.01)$ were reported in the loop ileostomy group. Patients with an ileostomy, however, more often presented with a high-output stoma $(p<0.01)$ resulting in dehydration $(p=0.03)$.

\section{Conclusion}

A loop ileostomy has a number of advantages over a colostomy. However, in patients with an increased risk for developing dehydration or compromised renal function, colostomy construction should be seriously considered given the higher complication risk in case of a high-output stoma. 


\section{INTRODUCTION}

In the Netherlands, stoma construction is one of the most commonly performed abdominal surgeries. About 9000 new stomas are constructed each year, resulting in a total of 38.000 patients with a stoma in the Netherlands alone. ${ }^{1}$

Stoma construction can be performed for several reasons; anastomotic leakage, severe perianal fistulas, obstructive colon cancer or a slow transit colon. The most common indication, however, is protection of a newly constructed colonic anastomosis. ${ }^{2}$ Several studies show a significant decrease in the number of clinically relevant anastomotic leakages when a deviating stoma is constructed. ${ }^{3}$

When a stoma is constructed, the surgeon has to decide which type of stoma will be constructed; a loop ileostomy, a loop colostomy, an end ileostomy or an end colostomy. The choice between a loop or end stoma is relatively simple. When the stoma is thought to be temporary, a loop stoma should always be preferred over an end stoma, since reversal of a loop stoma is technically less difficult. ${ }^{4}$

The choice between an ileostomy or a colostomy, however, is more difficult. When a patient presents with left-sided obstructive colon cancer, a colostomy should always be constructed. The ileocaecal valve prohibits retrograde movement of feces, making adequate colonic decompression in these patients impossible. Inadequate decompression can lead to life threatening complications, such as a colonic perforation and should always be prevented.

Whether a colostomy or ileostomy should be constructed is not as clear for other indications. The current Dutch guidelines do not recommend one stoma type over the other. ${ }^{5}$ The American Society of Colon and Rectal Surgeons hints towards a slight preference for ileostomy construction, since reversal is thought to be easier and stoma prolapse is less common. However, they also state that a loop ileostomy is more likely to result in a high output stoma, which could result in dehydration. Therefore, no clear preference is stated. ${ }^{6}$

The aim of the current study was to investigate which type of loop stoma should be preferred in patients receiving a temporary stoma and in whom it is technically possible to construct both types. This was investigated through systematic review of all available literature and a retrospective analysis of our own data.

\section{METHODS}

\section{Systematic literature research}

A systematic literature search was performed according to the 'Preferred reporting items for systematic reviews and meta-analysis (PRISMA) guidelines. ${ }^{7}$ Pubmed, Embase and Cochrane Library were searched on October $6^{\text {th }} 2016$ with the following search terms '(colostomy[tiab] OR transverstomy[tiab] OR ostomy[tiab] OR stoma[tiab] OR colostoma[tiab]) AND (ileostomy[tiab] OR blowhole[tiab] OR ileostoma[tiab])'. In addition, all reference lists were manually screened for additional relevant studies. 
After removal of all duplicates, two independent authors (FA and TB) screened all articles on title and abstract. In case of disagreement, the articles were discussed until consensus was reached. Literature was judged based on quality of evidence, where meta-analyses and systematic reviews were included first, followed by randomized controlled trials (RCT) and non-randomized, original studies. When an original study was already included in a metaanalysis or systematic review, the study was excluded.

All articles comparing a temporary loop ileostomy with a loop colostomy were included. For systematic reviews, the risk of bias was assessed using the 'Quality of reporting of metaanalysis' (QUOROM)-criteria, for RCTs the 'Consolidated standards of reporting trials' (CONSORT) criteria were used. Non-randomized studies were assessed using the 'Methodological index for non-randomized studies' (MINOR) criteria.

Two authors performed data collection independently. All demographical and clinical data, data on methodological quality, outcomes following stoma construction and outcomes on quality of life were collected.

\section{Retrospective analysis}

In addition to the literature search; data on all patients that underwent stoma construction between 2009 and 2015 in our hospital was collected. Patients were identified using the surgical code and data was collected from the electronic patient records. Age, gender, ASAscore and all stoma related outcomes were gathered. Stoma related outcomes included all complications that occurred while the stoma was in situ, such as high-output $(>1500 \mathrm{cc} / 24 \mathrm{~h}$ stoma production), dehydration, renal failure, prolapse, parastomal hernia, stoma retraction or obstruction, as well as all complications following stoma reversal, such as wound infection, anastomotic leakage, ileus or obstruction, incisional hernia and mortality. Complications were classified according to the Clavien-Dindo classification. Grade $3 \mathrm{~b}$ or higher was defined as major morbidity, whereas grade 3a or lower was considered minor morbidity.

\section{Systematic analysis}

Statistical analyses were conducted using SPSS 23.0. Continuous variables were reported as mean with standard deviation (SD) and compared using the independent t-test. Categorical variables were reported as exact numbers with percentages and compared between groups using the Fisher exact test. A difference was considered statistically significant when the twosided $\mathrm{p}$-value was $<0.05$ of when the confidence interval no longer included 1.0.

\section{RESULTS}

\section{Systematic literature research}

Figure one shows the results of the literature search. Following exclusion of all duplicates, 2092 articles remained for title and abstract screening. Twenty-seven studies were screened full text, where after nineteen were excluded for analysis. Sixteen were excluded because they 
FIGURE 1 | Flowchart of literature search, conducted on October $26^{\text {th }} 2016$, and consequent article selection.

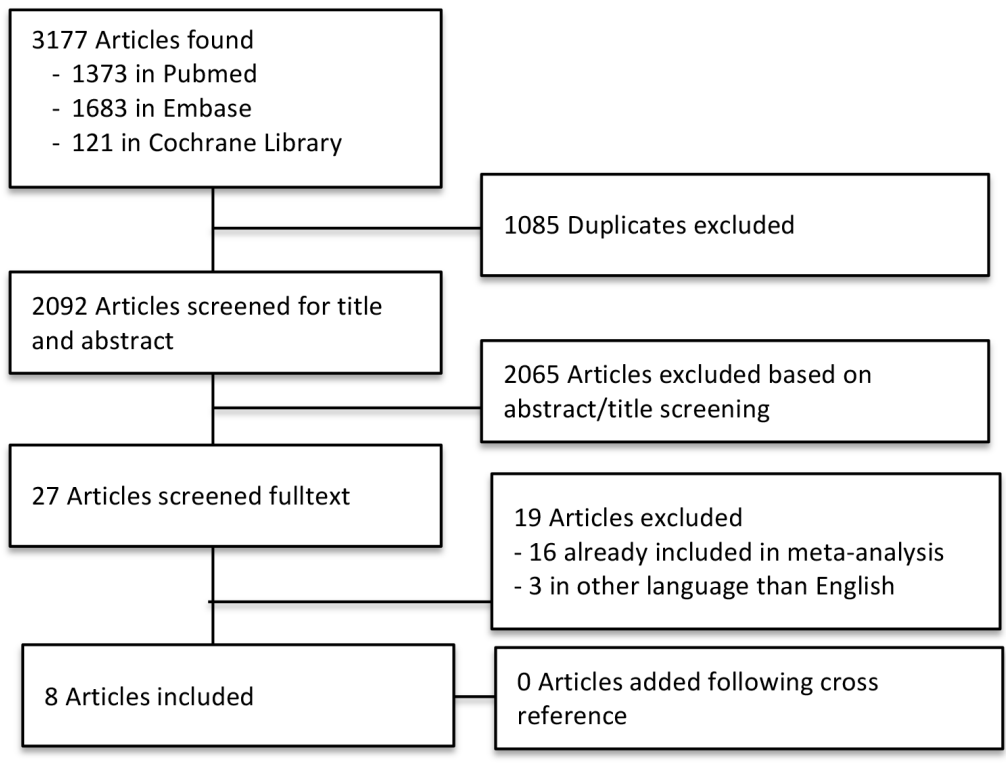

were already included in one of the meta-analyses and the other three studies were excluded because they were not written in English or Dutch. Cross-reference of all reference lists did not yield any additional articles.

In total, eight articles were included. All included studies compared a loop ileostomy with a loop colostomy. The primary and secondary outcome measures differed between the different studies.

Five meta-analyses reported on short-term outcomes, one systematic review reported longterm outcomes (incisional hernia's) following stoma reversal and two prospective studies reported on quality of life as a primary outcome measure (Table 1).

All studies were published between 2000 and 2014, with a range in the level of Evidence from $1 \mathrm{~A}$ to $2 \mathrm{~B} .{ }^{12}$ Table two shows a detailed critical appraisal of the methodology of all included studies. Five meta-analyses only included patients with a deviating stoma proximal of a colorectal anastomosis, ${ }^{13-17}$ one of these studies even limited inclusion to only patients that had undergone a low anterior resection for rectal carcinoma. ${ }^{13}$ The two studies investigating quality of life included patients with a stoma for any indication. ${ }^{18,19}$ In addition, the systematic review with incisional hernia's following stoma reversal as primary outcome measure also included patients with any indication for stoma construction. ${ }^{20}$ 


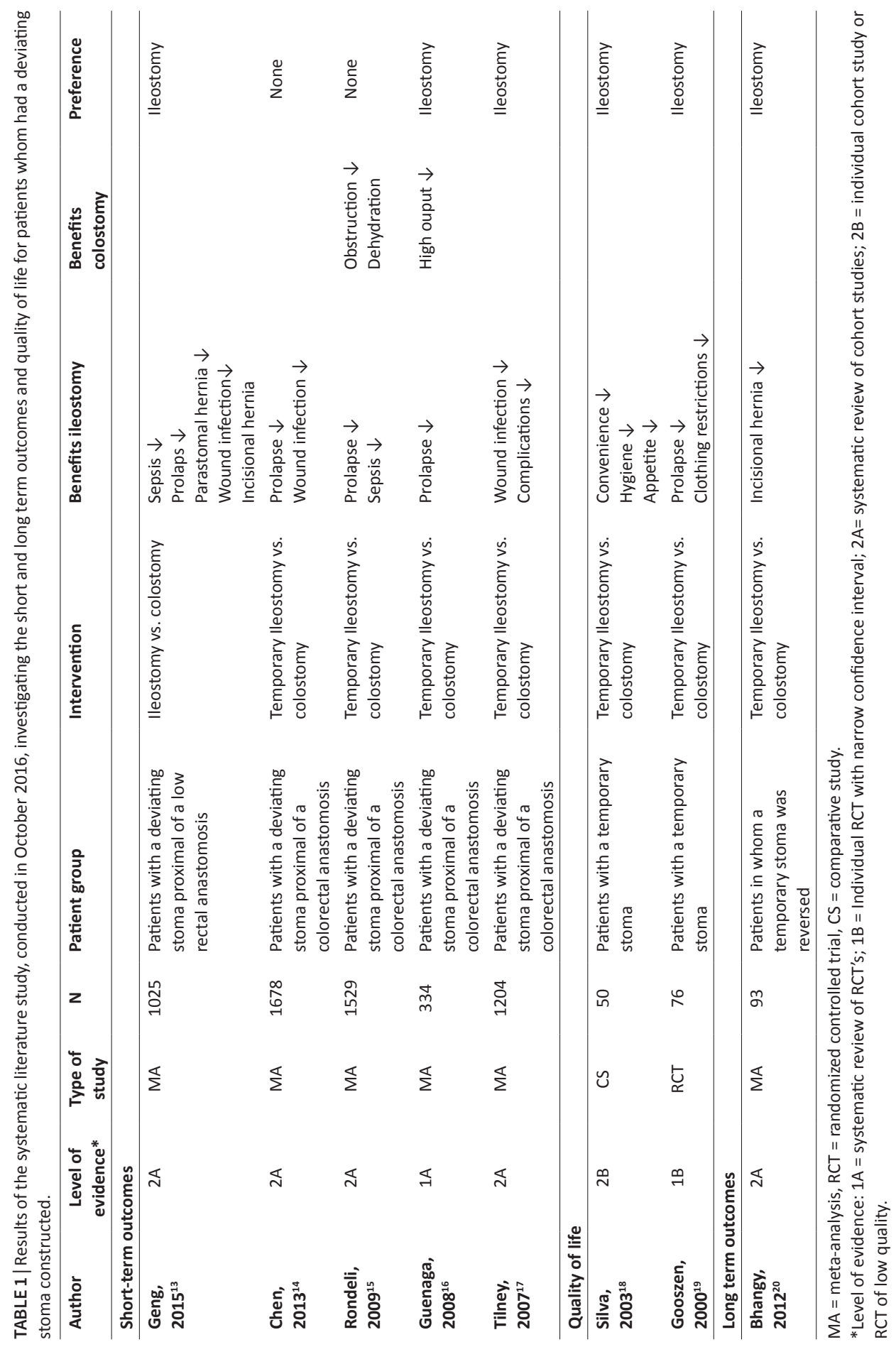




\section{Short-term outcomes}

Out of the five meta-analyses that investigated short-term outcomes following ileostomy or colostomy construction, three report an overall preference for ileostomy construction. ${ }^{13,16,17}$ An ileostomy is associated with fewer complication, prolapses, parastomal hernia's and wound infections. The two studies that did not specifically indicate a preference, found a lower incidence of high output stomas and dehydration with a colostomy.

\section{Quality of life}

Both studies that investigated quality of life concluded that a stoma negatively influences quality of life, independent of the stoma type. ${ }^{18,19}$ In some patients, however, an ileostomy seems to affect quality of life less than a colostomy. An ileostomy seems to be more hygienic, has less influence on appetite and requires fewer clothing adjustments. Influence on traveling or sexual activity was similar for both stoma types.

\section{Long-term outcomes}

Follow-up is limited in most studies. Only one large meta-analysis is available that compares the number of incisional hernia's following stoma reversal of both stoma types. The risk of an incisional hernia was substantially lower after ileostomy reversal compared to colostomy reversal with an odds ratio of $0.28(95 \% \mathrm{Cl} 0.12-0.65)$.

\section{Results from the retrospective analysis}

Between January 2009 to December 2015, a deviating stoma was constructed in 361 patients; in 179 patients a loop ileostomy was constructed, versus 182 patients (50.4\%) with a colostomy. Protection of the colorectal anastomosis was the indication for stoma construction in $76.5 \%$ of the patients $(n=274)$. Other indications were peri-anal fistulas $(15.0 \%, n=54)$ or anastomotic leakage $(9.1 \%, n=33)$ (Table 3$)$.

Mean age in the ileostomy group was 61.6 years (SD 14.1) and 60.4 years (SD 14.8) in the colostomy group. This age difference, as well as the baseline differences in gender and ASAscore, were not statistically significant.

\section{Results following stoma construction}

The number of stoma related complications was not significantly different when ileo- and colostomy were compared (69/182 patients (37.9\%) versus 67/179 (37.4\%) patients, respectively $(p=0.93))$. When looking at the specific complications separately, however, some significant differences were found. Patients with an ileostomy significantly more often had a high output stoma $(16.1 \%$ vs. $5.0 \%, p<0.01)$. This also results in a significantly higher dehydration rate in the ileostomy group $(12.9 \%$ vs. $3.4 \%, p=0.03)$. In the colostomy group, however, significantly more retractions were reported $(3.4 \%$ vs. $0.0 \%, p<0.01)$. All other shortterm outcomes, such as parastomal hernia rate, prolapse and stoma obstruction were not significantly different. In addition, the number of stomas that was not reversed was not different in both groups. 
TABLE 2A | Critical appraisal of the level of evidence of systematic reviews, according to the QUORUM-criteria ${ }^{10}$

\begin{tabular}{|c|c|c|c|c|c|c|}
\hline \multirow{2}{*}{$\begin{array}{l}\text { Criteria } \\
\text { Author, year }\end{array}$} & \multicolumn{6}{|c|}{ Critical appraisal } \\
\hline & $\begin{array}{l}\text { Geng, } \\
2015^{13}\end{array}$ & $\begin{array}{l}\text { Chen, } \\
2013^{14}\end{array}$ & $\begin{array}{c}\text { Rondelli, } \\
2009^{15}\end{array}$ & $\begin{array}{c}\text { Guanaga, } \\
\text { 2008 }^{16}\end{array}$ & $\begin{array}{l}\text { Tilney, } \\
2007^{17}\end{array}$ & $\begin{array}{c}\text { Bhangy, } \\
2^{2012^{20}}\end{array}$ \\
\hline Clear research question & + & + & + & + & + & + \\
\hline Adequate search & + & $?$ & + & + & + & + \\
\hline Reproducibility & + & + & + & - & $?$ & + \\
\hline Publication bias & + & + & + & + & + & ? \\
\hline Clear in- and exclusion criteria & + & + & + & + & + & + \\
\hline Methodologic quality & + & - & + & + & + & + \\
\hline Baseline characteristics & + & + & + & - & + & + \\
\hline Meta-analysis conducted correctly & + & + & + & + & + & + \\
\hline Comparable results & + & + & + & + & + & + \\
\hline Relevant effect size & + & + & + & + & + & + \\
\hline Confidence interval & + & + & + & + & + & + \\
\hline Level of evidence & $2 a$ & $2 a$ & $2 a$ & $1 a$ & $2 a$ & $2 a$ \\
\hline
\end{tabular}

Legend: + = good, - = poor, ? = unclear

TABLE 2B | Critical appraisal of the level of evidence of randomized controlled trials according to the CONSORT criteria $^{11}$

\begin{tabular}{lc}
\hline Criteria & $\begin{array}{c}\text { Critical appraisal } \\
\text { Gooszen, 2014 }\end{array}$ \\
\hline Clear research question & $?$ \\
Randomization & + \\
Blinding & + \\
Clear in- and exclusion criteria & - \\
Comparable groups & + \\
Comparable interventions & + \\
Clear outcomes & + \\
Loss-to-follow up described & + \\
Relevant effect size & + \\
Confidence interval & + \\
General izable & + \\
\hline Level of evidence & $1 \mathrm{~B}$ \\
\hline
\end{tabular}

TABLE 2C | Critical appraisal of the level of evidence of comparative studies (prospective). according to the MINORS criteria ${ }^{12}$

\begin{tabular}{ll}
\hline Criteria & Critical appraisal \\
Author, year & Silva, 2003 \\
\hline Design & + \\
Clear aim & $?$ \\
Consecutive patient inclusion & + \\
Prospective data & + \\
Relevant outcome measures & + \\
Bias & + \\
Adequate follow-up length & + \\
Loss-to-follow-up $<5 \%$ & + \\
Sample size calculated & - \\
\hline Level of evidence & $2 \mathrm{~B}$ \\
\hline
\end{tabular}

TABLE 3 | Indications for deviating stoma construction in 361 patients at the Meander Medical Center Amersfoort between January 2009 and December 2015.

\begin{tabular}{lc}
\hline Indication & $\mathbf{N}(\%)$ \\
\hline Protection of a colorectal anastomosis & $\mathbf{2 7 4}(\mathbf{7 6 . 5 )}$ \\
Colostomy & $116(42.3)$ \\
Ileostomy & $158(57.7)$ \\
Peri-anal fistulas or anal carcinoma & $\mathbf{5 4 ( 1 5 . 0 )}$ \\
Colostomy & $40(74.1)$ \\
lleostomy & $14(25.9)$ \\
Anastomotic leakage & $\mathbf{3 3 ( 9 . 1 )}$ \\
Colostomy & $23(69.7)$ \\
lleostomy & $-10(30.3)$ \\
\hline
\end{tabular}




\section{Results following stoma reversal}

The number of overall, but also major complications did not differ between the two stoma types. In addition, 30-day mortality, the number of wound infections, anastomotic leakages and postoperative cases of ileus were equal for patients that underwent ileostomy or colostomy reversal. On the long-term, however, patients that had a colostomy reversed were significantly more likely to develop an incisional hernia $(27.4 \%$ vs. $14.8 \%, p<0.01)$.

\section{DISCUSSION}

\section{Outcomes}

The results of our literature search indicated that an ileostomy should be preferred over a colostomy because of the favorable short and long-term outcomes. Patients with an ileostomy have fewer wound infections, stoma prolapses, stoma retractions, parastomal hernia's and incisional hernias and report a better quality of life. The results from the data in our own hospital underline these findings; stoma retraction was significantly less common in the ileostomy group. It should however, be taken into account that both the literature search as well as the results from our own data indicate that having an ileostomy increases the risk of a high output stoma and dehydration as a consequence hereof.

Interestingly, our own data show that currently, in $50 \%$ of the cases an ileostomy is constructed and in $50 \%$ of the cases a colostomy. This reflects the current state of clinical uncertainty, where the type of stoma constructed varies per hospital or even per surgeon. Patients that present with perianal fistulas, anal carcinoma or anastomotic leakage as indication for stoma construction were more likely to have a colostomy (70\%) constructed compared to an ileostomy (30\%) in our hospital. A possible explanation for the higher colostomy rate in these patients might be that a colostomy can be constructed in a minimally invasive way using the trephine method. According to this method, a transverse colon loop is externalized through a small incision in the upper right abdominal quadrant, where after the stoma can be constructed. ${ }^{21}$ For ileostomy construction, more anatomical orientation is needed requiring a laparotomy or laparoscopy. ${ }^{22}$

Both the literature search, as well our own results indicate that an ileostomy is associated with an increased risk for high-output and dehydration. In most cases, this can be managed conservatively with medication and severe complications are rare. This is substantiated by the fact that the incidence of renal failure was not significantly different between ileo- and colostomy patients. However, in some instances, a high-output stoma can be a dangerous and even life-threatening complication. An important risk factor for dehydration is fluid depletion, which is more common in patients that are not self-sufficient, such as patients with dementia, patient with mental retardation and patients that are bedridden. In addition, patient with a pre-existent compromised renal function are at higher risk of (severe) complications of a highoutput stoma. ${ }^{23}$ Lastly, there are suggestions that adjuvant chemotherapy, and specifically capecitabine, increases the chance of chemotherapy induced diarrhea and high-output stoma 
TABLE 4 | Baseline characteristics and outcomes for patients

\begin{tabular}{|c|c|c|c|}
\hline Variable & $\begin{array}{c}\text { Ileostomy; } \mathbf{n}(\%)^{*} \\
\mathrm{~N}=182\end{array}$ & $\begin{array}{c}\text { Colostomy; } \mathbf{n}(\%)^{*} \\
\text { N = } 179\end{array}$ & Odds ratio $(95 \% \mathrm{Cl})^{\mathrm{a}}$ \\
\hline Age in years (SD) & $61.6(14.1)$ & $60.4(14.8)$ & - \\
\hline Male gender & $100(54.9)$ & $91(50.8)$ & \\
\hline \multicolumn{4}{|l|}{ ASA-score } \\
\hline$I-I I$ & $164(90.1)$ & $162(90.5)$ & \\
\hline III-IV & $18(9.9)$ & $17(9.5)$ & \\
\hline \multicolumn{4}{|l|}{ While stoma is in place } \\
\hline Complication & 69 (37.9) & $67(37.4)$ & $0.7(0.6-1.4)$ \\
\hline High output & $24(16.1)$ & $6(5.0)$ & $3.8(1.6-9.0)$ \\
\hline Dehydration & $17(12.9)$ & $4(3.4)$ & $3.7(1.2-11.4)$ \\
\hline Renal failure & $10(6.7)$ & $4(3.4)$ & $2.0(0.6-6.8)$ \\
\hline Prolapse & $6(4.7)$ & 9 (12.9) & $0.5(0.2-1.5)$ \\
\hline Parastomal hernia & $10(6.7)$ & $10(8.5)$ & $0.7(0.3-1.9)$ \\
\hline Obstruction & $12(7.9)$ & $7(5.9)$ & $1.4(0.5-3.5)$ \\
\hline Retraction & $0(0)$ & $4(3.4)$ & $0.01(0.01-0.02)$ \\
\hline Permanent stoma & $4(2.2)$ & $8(4.5)$ & $2.0(0.6-7.0)$ \\
\hline \multicolumn{4}{|l|}{ Following stoma reversal } \\
\hline Complication & $33(18.1)$ & $33(19.5)$ & $0.9(0.5-1.6)$ \\
\hline Major complication & $16(8.8)$ & $15(80.7)$ & $1.0(0.5-2.1)$ \\
\hline 30-day mortality & $1(0.5)$ & $1(0.5)$ & $1.0(0.1-15.8)$ \\
\hline Wound infection & $8(4.4)$ & $13(7.6)$ & $0.5(0.2-1.4)$ \\
\hline Anastomotic leakage & $2(1.1)$ & $3(1.7)$ & $0.6(0.1-3.8)$ \\
\hline Obstruction & $13(7.1)$ & $7(4.1)$ & $1.8(0.7-4.6)$ \\
\hline Incisional hernia & $27(14.8)$ & $49(27.4)$ & $0.5(0.3-0.8)$ \\
\hline
\end{tabular}

*Unless stated differently. ${ }^{a}$ Colostomy is reference. Significant differences $(p<0.05)$ are bold. Abbreviations: ASA-score; American Society of Anesthesiologists.

when an ileostomy is present. ${ }^{24,25}$ When choosing between an ileostomy and colostomy, the physician should therefore always weigh the (dis-)advantages of both approaches in the light of the individual patient and its future treatment perspectives.

The results of our retrospective analysis showed a complication rate of $40 \%$ in patients with a stoma, irrespective of the stoma type. In a previous study in our hospital, where all complications were registered prospectively, a percentage over $80 \%$ was found. ${ }^{26}$ This difference is probably due to the fact that our data collection was retrospective. Mild complications, such as skin irritation, leakage and material problems might not always be documented. When interpreting these results, it is therefore important to realize that the reported complication rate might be an underestimation.

\section{Limitations}

A limitation of the current study is its retrospective design, since this increases the chance of selection bias. The number of patients $(n=361)$ and the fact that patients were included consecutively, however, might have reduced this risk. A second limitation is the heterogeneity of the study cohort. In order to generate a widely applicable advice, we choose to include all patients with a stoma between 2009-2015, independent of the indication for stoma 
construction. However, because of this heterogeneity, some information about the number of complications following stoma construction is not available. In addition, the type of surgery the patients underwent while the stoma was constructed differed substantially. For some patients, stoma construction was the only intervention, while stoma construction was only part of a larger surgery in others.

A loop ileostomy has several advantages over a loop colostomy. A loop ileostomy should therefore be preferred. However, two exceptions should be taken into account when a colostomy should be constructed; 1 ) in patients with left-sided obstructive colon cancer, since adequate colonic decompression is of great importance and; 2 ) in patients with an increased risk of dehydration or compromised renal function (figure 2).

FIGURE 2 | Helpful flowchart in deciding which stoma type to construct.

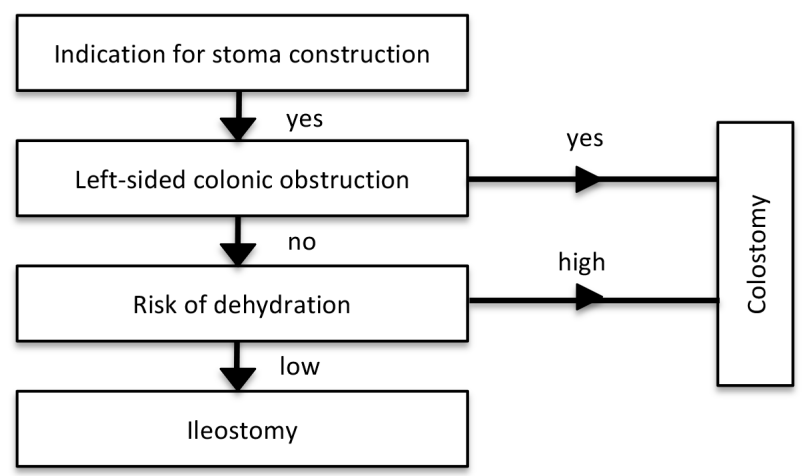




\section{REFERENCES}

1. Wat is een stoma? Bergambacht: Stichting Stomaatje; 2005-2013. www.stomaatje.nl/watisnueenstoma/ watiseenstoma.html, accessed Oct 6th 2016

2. Scott-Conner, CEH. Chassin's operative strategy in colon and rectal surgery. New York: Springer; 2006:18

3. Hüser N, Michalski CW, Erkan M, et al. Systematic review and metaanalysis of the role of defunctioning stoma in low rectal cancer surgery. Ann Surg. 2008;248:52-60

4. Bruns BR, DuBose J, Pasley J, et al. Loop versus end colostomy reversal: has anything changed? Eur $J$ Trauma Emerg Surg. 2015;41:539-543.

5. Landelijke werkgroep Gastro Intestinale Tumoren. Richtlijn colorectaal carcinoom, versie 3.0. Utrecht: IKNL; 2014

6. Monson JRT, Weiser MR, Buie WD, Chang GJ, Rafferty JF. Practice parameters for the management of rectal cancer (revised). Dis Colon Rectum. 2013;56:535-50

7. Moher D, Liberati A, Tetzlaff J, Altman DG; PRISMA Group. Preferred reporting items for systematic reviews and meta-analyses: the PRISMA statement. J Clin Epidemiol. 2009;62:1006-12

8. Moher D, Cook DJ, Eastwood S, Olkin I, Rennie D, Stroup DF. Improving the quality of reports of meta-analyses of randomised controlled trials: the QUOROM statement. Lancet. 1999;354:1896-900

9. Moher D, Schulz KF, Altman DG; CONSORT GROUP (Consolidated Standards of Reporting Trials). The CONSORT statement: revised recommendations for improving the quality of reports of parallel-group randomized trials. Ann Intern Med. 2001;134:657-62

10. Slim K, Nini E, Forestier D, Kwiatkowski F, Panis $\mathrm{Y}$, Chipponi J. Methodological index for non-randomized studies (minors): development and validation of a new instrument. ANZ J Surg. 2003;73:712-6

11. Clavien PA, Barkun J, de Oliveira ML, et al. The ClavienDindo classification of surgical complications: five-year experience. Ann Surg. 2009;250:187-96.

12. OCEBM Levels of Evidence Working Group. The Oxford 2011 levels of evidence. Oxford: Oxford Centre for Evidence-Based Medicine; 2011. www.cebm.net/ ocebm-levels-of-evidence/, geraadpleegd oktober 2016

13. Geng HZ, Nasier D, Liu B, Gao H, Xu YK. Meta-analysis of elective surgical complications related to defunctioning loop ileostomy compared with loop colostomy after low anterior resection for rectal carcinoma. Ann R Coll Surg Engl. 2015;97:494-501
14. Chen J, Wang DR, Zhang JR, Li P, Niu G, Lu Q. Metaanalysis of temporary ileostomy versus colostomy for colorectal anastomoses. Acta Chir Belg. 2013;113:330-9

15. Rondelli F, Reboldi P, Rulli A, et al. Loop ileostomy versus loop colostomy for fecal diversion after colorectal or coloanal anastomosis: a metaanalysis. Int I Colorectal Dis. 2009;24:479-88

16. Güenaga KF, Lustosa SA, Saad SS, Saconato H, Matos D. lleostomy or colostomy for temporary decompression of colorectal anastomosis. Systematic review and metaanalysis. Acta Cir Bras. 2008;23:294-303

17. Tilney HS, Sains PS, Lovegrove RE, Reese GE, Heriot AG, Tekkis PP. Comparison of outcomes following ileostomy versus colostomy for defunctioning colorectal anastomoses. World J Surg. 2007;31:1142-51

18. Silva MA, Ratnayake G, Deen KI. Quality of life of stoma patients: temporary ileostomy versus colostomy. World J Surg. 2003;27:421-4

19. Gooszen AW, Geelkerken RH, Hermans J, Lagaay MB, Gooszen HG. Quality of life with a temporary stoma: ileostomy vs. colostomy. Dis Colon Rectum. 2000;43:650-5

20. Bhangu A, Fletcher L, Kingdon S, Smith E, Nepogodiev D, Janjua U. A clinical and radiological assessment of incisional hernias following closure of temporary stomas. Surgeon. 2012;10:321-5

21. Nylund G, Oresland T, Hultén L. The trephine stoma: formation of a stoma without laparotomy. Eur J Surg. 1997;163:627-9

22. Kini SU, Perston Y, Radcliffe AG. Laparoscopically assisted trephine stoma formation. Surg Laparosc Endosc. 1996;6:371-4

23. Messaris E, Sehgal R, Deiling $S$, et al. Dehydration is the most common indication for readmission after diverting ileostomy creation. Dis Colon Rectum. 2012;55:175-80

24. Robertson JP, Wells $\mathrm{Cl}$, Vather R, Bissett IP. Effect of Diversion lleostomy on the Occurrence and Consequences of Chemotherapy-Induced Diarrhea. Dis Colon Rectum. 2016;59:194-200

25. Oliphant R, Czerniewski A, Robertson I, McNulty C, Waterston A, Macdonald A. The effect of adjuvant chemotherapy on stoma-related complications after surgery for colorectal cancer: a retrospective analysis. J Wound Ostomy Continence Nurs. 2015;42:494-8

26. Formijne Jonkers HA, Draaisma WA, Roskott AM, van Overbeeke AJ, Broeders IA, Consten EC. Early complications after stoma formation: a prospective cohort study in 100 patients with 1-year follow-up. Int J Colorectal Dis. 2012;27:1095-9 


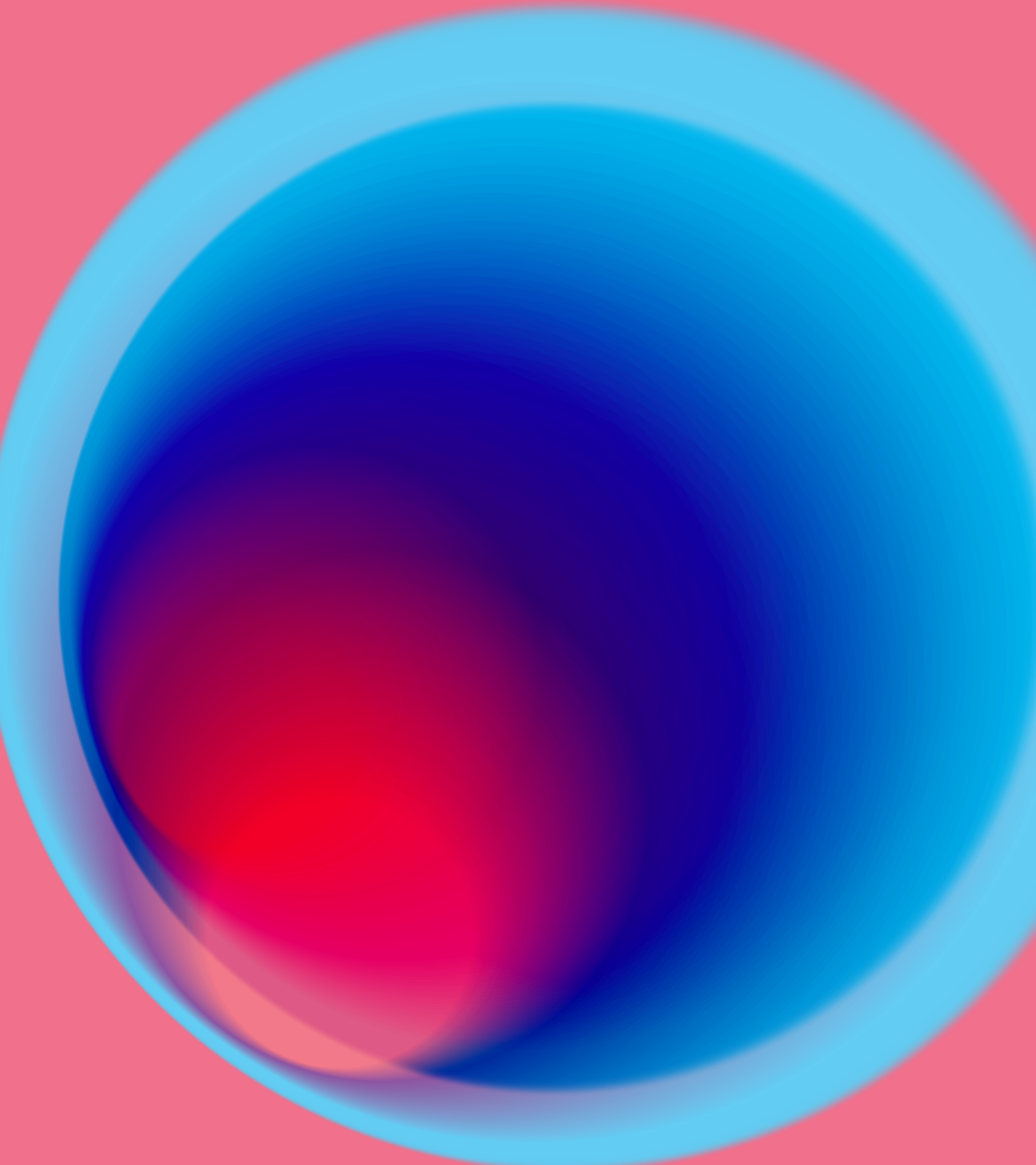





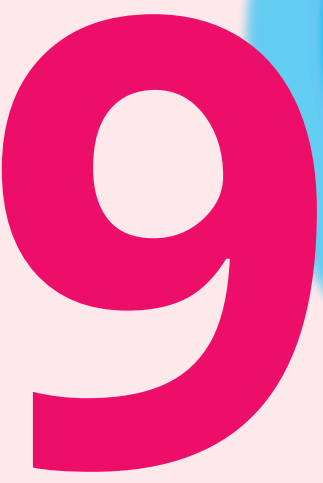

\section{Long-term outcomes following stent placement as bridge to surgery compared to emergency surgery in patients with acute left-sided colonic obstruction; an updated meta-analysis}

Critical Reviews in Oncology Hematology 2018

\section{F.J. Amelung}

T.A. Burghgraef

P.J. Tanis

J.E. van Hooft

F. ter Borg

P.D. Siersema

W.A. Bemelman

E.C.J. Consten 


\section{ABSTRACT}

\section{Background}

Controversy remains on the role of self-expandable metal stent (SEMS) placement as bridge to surgery (BTS) in curative patients with obstructive colon cancer. Hesitance mainly relates to uncertainness about the long-term oncological outcomes. The aim of this meta-analysis was to determine the long-term oncological outcomes of SEMS as BTS versus emergency surgery (ES).

\section{Methods}

This systematic review was performed using the PRISMA guidelines. Pubmed, Embase and Cochrane Library were searched without restrictions. All studies comparing SEMS as BTS with ES reporting on long-term oncological outcomes and with a follow-up of $>2$ years were included. Methodological quality was assessed using the Cochrane Collaborations tool or the ROBINS-I tool, depending on study design. The main outcome was three- and five year overall survival. Pooled odds ratios (OR) with $95 \%$ confidence intervals were calculated using a random effects model.

\section{Results}

Twenty-one comparative studies including five RCTs were selected, reporting on a total of 1919 patients; 938 in the SEMS group versus 981 in the ES group. Meta-analysis showed no significant difference regarding three- and five-year overall survival (OR:0.85, (0.68-1.08) and OR:1.04 (0.68-1.57)), three- and five-year disease-free survival (OR:0.96 (0.73-1.26) and OR:0.97 (0.71$1 \cdot 33))$ and the number of local recurrences (OR:1.32 (0.78-2.23)). Sensitivity analysis revealed significantly better survival in the SEMS group in studies with $\geq 40$ SEMS procedures (OR:0.73; $0.55-0.97$ ) or when perforation rate was <8\% (OR:0.71 (0.52-0.97)). An opposite trend was observed in RCTs (OR:1.18 (0.66-2.11)).

\section{Conclusion}

This meta-analysis suggests that SEMS placement as BTS is oncologically safe, especially in experienced hands, but available data do not allow for a definitive conclusion because of the risk of bias from the majority of non-randomized studies. 


\section{INTRODUCTION}

Traditionally, acute malignant colonic obstruction has been managed with emergency surgery (ES), either consisting of acute resection or decompressing stoma construction. Resection in the emergency setting might be associated with a substantial risk of mortality and morbidity rates, especially in patients with high operative risk. ${ }^{1-3}$ This resulted in the search for alternative treatment approaches. In 1991, Dohmoto first proposed the placement of a self-expandable metallic stent (SEMS) to relief colonic obstruction. ${ }^{4}$ SEMS placement potentially enables an elective (laparoscopic) resection in a patient with an optimized clinical condition with the potential to avoid a stoma.

Almost 30 years after its first introduction, SEMS placement for left-sided obstructing colon cancer is still surrounded by controversy. Initial meta-analyses of cohort studies comparing SEMS as bridge to surgery (BTS) with ES showed favorable short-term outcomes in the SEMS group. ${ }^{5,6}$ Several randomized controlled trials (RCTs) have been performed thereafter, but were often prematurely terminated because of stent-related complications. Meta-analyses of these RCTs could not confirm an impact of SEMS on postoperative mortality, but did reveal a higher chance of undergoing laparoscopic surgery, lower morbidity rates, fewer temporary stoma constructions and higher primary anastomoses rates. ${ }^{7-11}$

Even though the short-term benefits of SEMS as BTS have been established in recent years, many physicians remain hesitant to place stents in a curative setting, since it has been suggested to negatively influence oncological outcomes. ${ }^{12}$ Concerns include an altered pathology after stent insertion. ${ }^{13-15}$ To what extent these histological findings translate into worse survival remains unclear. Additionally, concerns have been raised about the prognostic impact of a SEMS-related perforation. ${ }^{16,17}$ Since SEMS-related perforations occur in about $8 \%$ of the patients, it is important to further investigate the oncological consequences. ${ }^{18}$ Because of the on-going debate and the increasing body of relevant literature, this systematic review and meta-analysis was aimed to determine long-term oncological outcomes after SEMS as BTS compared to ES.

\section{METHODS}

This systematic review and meta-analysis was performed using the Preferred Reporting Items for Systematic Reviews and Meta-Analyses (PRISMA) guidelines to minimize risk of bias. ${ }^{19}$

\section{Search strategy and study selection}

A systematic literature search was conducted in MEDLINE (Pubmed), EMBASE (Ovid) and Cochrane Library. There were no restrictions to the search. The final search was conducted on December $20^{\text {th }}$ 2017. The search terms used were synonyms for stent and colonic obstruction. The exact search as used in EMBASE was: (stent:ab,ti OR SEMS:ab,ti OR 'bridge to surgery':ab,ti OR 'staged resection':ab,ti) AND ('acute obstruction':ab,ti OR ileus:ab,ti OR 'colon 
obstruction':ab,ti OR 'colonic obstruction' :ab,ti OR 'intestinal obstruction':ab,ti OR 'malignant obstruction':ab,ti OR 'rectosigmoidal obstruction':ab,ti OR 'left sided obstruction':ab,ti OR 'left-sided obstruction':ab,ti OR 'distal obstruction':ab,ti OR 'large bowel obstruction':ab,ti). MEDLINE and the Cochrane Library were searched using similar terms. Articles deemed potentially relevant were screened full text for inclusion by two authors (FA and TB) independently. In addition, references of all included studies were hand-searched for additional studies. Final inclusion of the articles was based on consensus.

\section{Inclusion/exclusion criteria}

In- and exclusion criteria were predefined. Studies were considered eligible for inclusion when they met the following criteria: 1) comparative study between SEMS placement as BTS and emergency surgery, 2) reporting on at least one long-term oncological outcome measure; overall survival, disease free survival and/or any type of recurrence, 3) all included patients in the studies had to be treated with curative intent, and 4) a median follow-up time of minimally two years. Studies were excluded if: 1) a language other than Dutch, English, German or French was used, or 2) there was no possibility to extract the exact numbers for outcome measures. In addition, conference abstracts without subsequent publication were excluded.

\section{Data extraction and study outcomes}

A priori, a data sheet was created and data were extracted independently by two authors (FA and TB). Disagreements were resolved by re-examination of the relevant study until consensus was reached; in case of disagreement a third author (EC) was involved for the final decision. For each included study, the primary author's name, publication year, design and duration of the study, number of included patients, median follow-up, and baseline characteristics were collected. Baseline characteristics entailed age, sex, tumor location, ASA-score, comorbidity, and adjuvant chemotherapy.

Primary outcomes were three and five-year overall and disease free survival. Overall recurrence rate, local recurrence rate, and permanent stoma rate were considered as secondary outcome measures. If the study provided only a Kaplan-Meier survival curve and not absolute survival rates, the rate was extracted as accurately as possible from the figure, and combined with the number of patients still in the study to reconstruct the number of events. In order to prevent overestimation of the precision of the effect measure, survival rates determined from a Kaplan Meier graph were only taken into account if at least two-third of the patients were still at follow-up after three and/or five years postoperatively. Whether more than two-third of the patients were still in follow-up after three and/or five years postoperatively was determined by counting the number of censored patients, or by looking at the median follow-up and its inter-quartile range. Local recurrence was defined as a recurrence at the anastomosis, tumor bed, mesentery, draining lymphatic system, surgical scar, or port sites. Overall recurrences included all cases of local recurrence as well as synchronous distant metastases. 


\section{Quality assessment}

All included studies were critically and independently appraised by two authors (FA and TB). For RCTs, the Cochrane Collaboration's tool for assessing the risk of bias was used. ${ }^{20}$ Observational comparative studies were appraised using the ROBINS-I tool for assessment of risk of bias. ${ }^{21}$

\section{Statistical analysis}

Data analyses were performed using Review Manager 5.3 software (the Cochrane Collaboration 2012, Denmark). Pooled odds ratios (OR) with $95 \%$ confidence intervals $(95 \% \mathrm{Cl})$ for overall survival, disease free survival, overall recurrence, local recurrence, and permanent stoma were calculated using a random effects model. The OR represents the odds of an adverse event (e.g. mortality or disease recurrence) occurring in the experimental group (SEMS as BTS) versus the control group (ES). An OR $<1$ favored the SEMS as BTS group. The point estimate OR was considered statistically significant when the $p$-value was $<0.05$ and the $95 \% \mathrm{Cl}$ did not include the value 1 . Heterogeneity among the included studies was assessed using graphical exploration of funnel plots, the Cochrane Q-statistic ( $p<0 \cdot 1$ was considered representative of statistically significant heterogeneity) and the $\mathrm{I}^{2}$ statistic $\left(\mathrm{I}^{2}>50 \%\right.$ was considered to represent substantial heterogeneity). ${ }^{22} \mathrm{~A}$ random-effects model was used for analysis given the variability of methods and populations in the included studies.. Furthermore, sensitivity analysis on three-year overall survival across six variables was conducted in order to investigate the robustness of the findings of this meta-analysis; variables included study design, region of the study, number of patients treated with SEMS, technical success rate of SEMS placement, publication year and perforation rate.

\section{RESULTS}

\section{Study selection}

The initial search yielded 2573 relevant articles. After removal of duplicates, 1913 studies remained, of which 1886 studies were excluded based on title and/or abstract (Figure 1). Eventually, the full text of 27 articles was evaluated, with eight more studies being excluded. Reasons for exclusion were; non-comparative study $(n=2)$, review article $(n=1)$, Chinese language $(n=1)$, comparison with elective surgery $(n=2)$, no exact data retrievable $(n=2)$. Two additional studies were added following manual cross-reference.

The included studies were published between 2003 and 2017, and reported on a total of 1919 patients: 938 in the SEMS as BTS group versus 981 in the ES group. ${ }^{12,14,17,23-40}$ The number of patients in the individual studies ranged from 26-240. Five studies were RCTs, ${ }^{17,25,26,29,37}$ four reported on prospectively collected data, ${ }^{27,28,30,36}$ and twelve studies had a retrospective design, ${ }^{12,14,23,24,31-35,38-40}$ Ten studies were conducted in Europe, ${ }^{12,17,24,25,28-30,33,36,37}$ and eleven in Asia. ${ }^{14,23,26,27,31,32,34,35,38-40}$ Median follow-up time ranged from 24 to 58 months. The baseline characteristics of each included study are shown in Table 1 . Nine studies had a low risk of bias, ${ }^{12,14,27,29,31,34-37}$ ten a moderate risk of bias, ${ }^{24,25,29,30,32,35,38,40}$ and two had a severe risk of bias. ${ }^{33,39}$ (Table 2 ) 


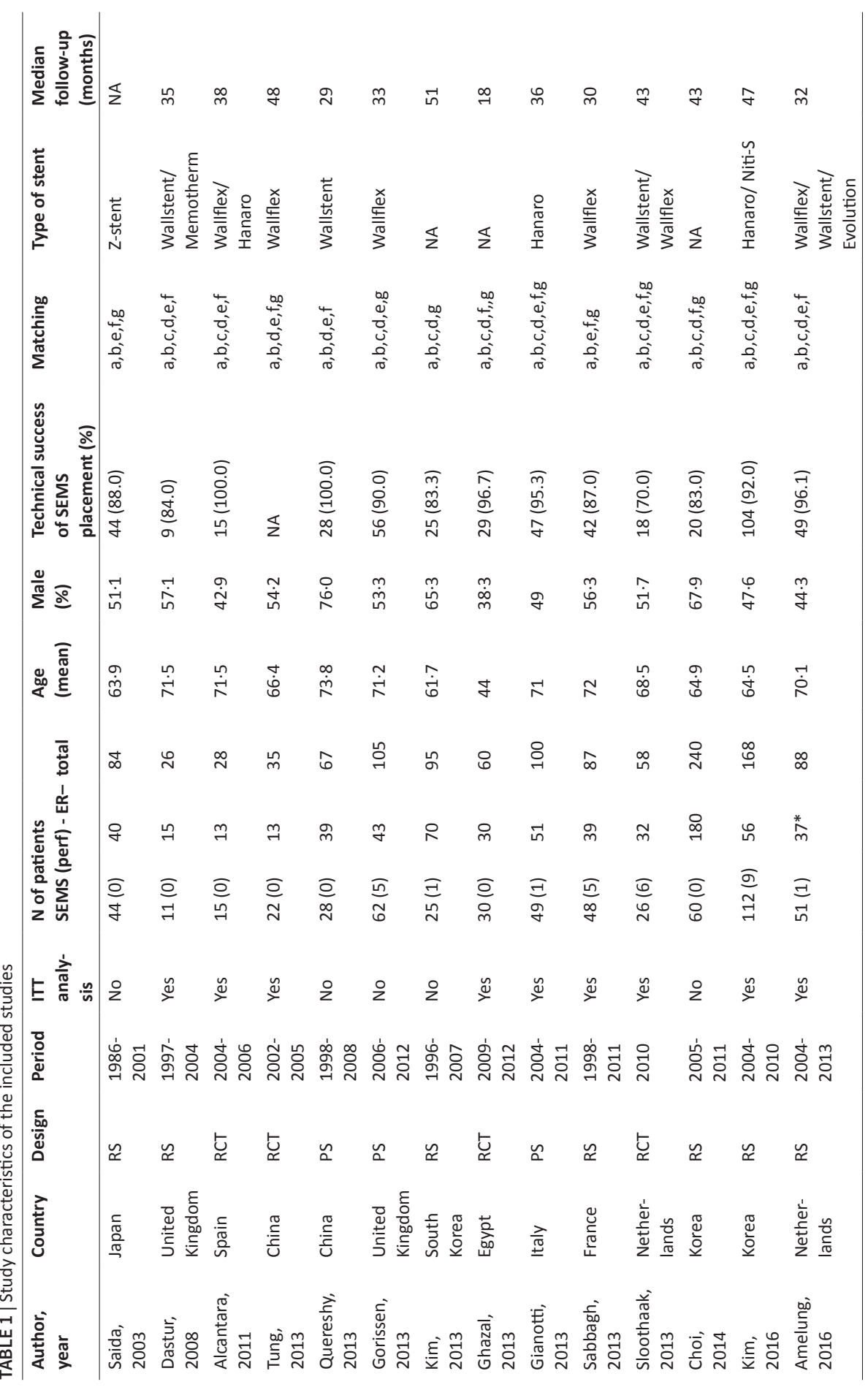




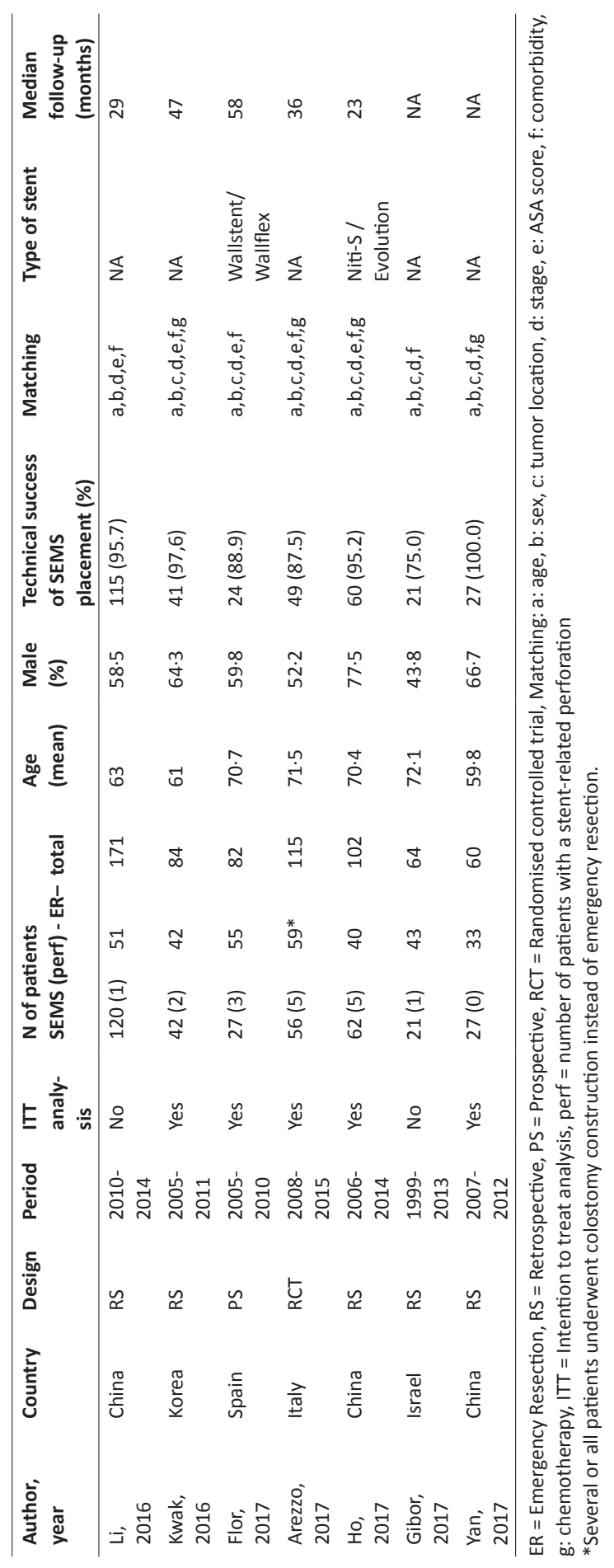


FIGURE 1 | Flowchart of literature search on December $20^{\text {th }} 2017$

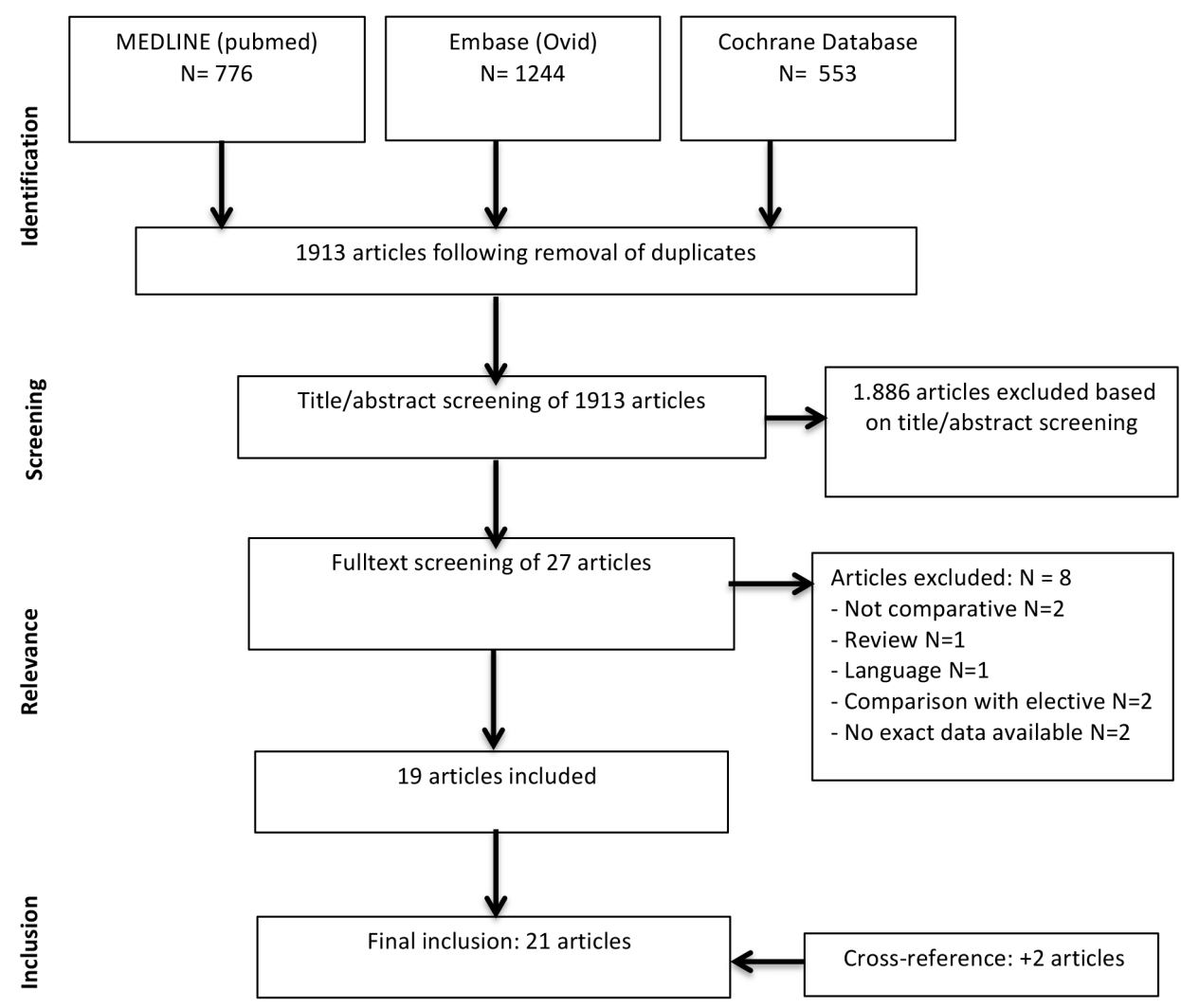

\section{Overall survival}

Of the twenty-one studies included in this meta-analysis, only Ghazal et al. did not report overall survival (OS) data. ${ }^{27}$ Meta-analysis of the pooled data did not show a significant difference in three-year survival rates when SEMS was compared to ES (69.7\% vs. $67.9 \%$, respectively; OR 0.85, 95\% $\mathrm{Cl} 0 \cdot 68-1 \cdot 08 ; 20$ studies, 758 vs. 797 patients). Moderate evidence of heterogeneity was found ( $p=0 \cdot 02, l^{2}=43 \%$ ) (Figure $2 A$ ). Five-year overall survival rate was retrievable from seven studies (230 vs. 283 patients), $14,24,28,32,33,36,39$ and was $63 \cdot 5 \%$ in the SEMS group versus $57.9 \%$ in the ES group. This difference was not significant (OR $1 \cdot 04,95 \% \mathrm{Cl} 0.68$ 1.57), without evidence of heterogeneity ( $\left.p=0.92, I^{2}=0 \%\right)$ (Figure $\left.2 B\right)$. Visual inspection of the funnel plot did not suggest the presence of publication bias (Figure $5 \mathrm{~A}$ ).

\section{Disease free survival}

Three-year disease-free survival (DFS) was reported in thirteen studies ( 525 vs. 582 patients), 12,14,26-28,30-35,36-38 five-year disease-free survival in six studies (206 vs. 306 patients). ${ }^{14,23,27,31,32,36}$ The three-year and five-year disease-free survival rates were $66 \cdot 1 \%$ and $63 \cdot 1 \%$ in the SEMS 
TABLE 2A | Risk of bias summary of the included studies. Quality assessment of RCTs using the Cochrane Collaborations Tool for assessment of risk of bias.

\begin{tabular}{|c|c|c|c|c|c|}
\hline & 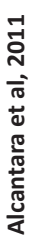 & 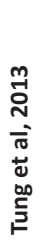 & $\begin{array}{l}m \\
\stackrel{n}{N} \\
\frac{N}{\pi} \\
\frac{0}{0} \\
\frac{N}{N} \\
\frac{\pi}{5}\end{array}$ & 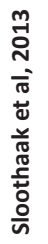 & 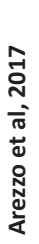 \\
\hline \multicolumn{6}{|c|}{ Random sequence generation } \\
\hline \multicolumn{6}{|c|}{ Allocation concealment } \\
\hline \multicolumn{6}{|c|}{ Blinding of participants and personnel } \\
\hline \multicolumn{6}{|c|}{ Blinding of outcome assessment } \\
\hline \multicolumn{6}{|c|}{ Incomplete outcome data } \\
\hline \multicolumn{6}{|c|}{ Selective reporting } \\
\hline \multicolumn{6}{|c|}{ Other bias } \\
\hline Overall & & & & & \\
\hline
\end{tabular}

Legend; Risk of bias: $\square$ Low, $\square$ Moderate, $\square$ Serious, $n$ Critical

TABLE 2B | Quality assessment of non-randomized studies using the ROBINS-I tool for assessment of risk of bias.

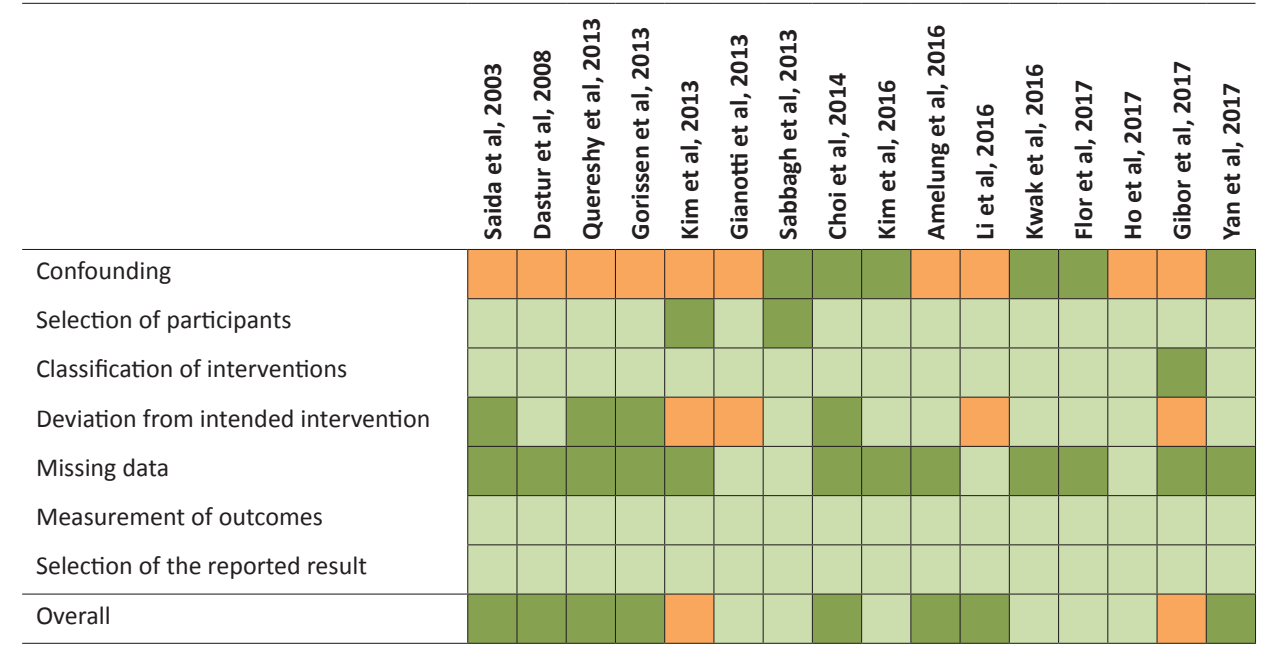

Legend; Risk of bias: $₫$ Low, $\square$ Moderate, $₫$ Serious, $\square$ Critical

group compared to $65.3 \%$ and $59.8 \%$ in the ES group, respectively. These rates were not

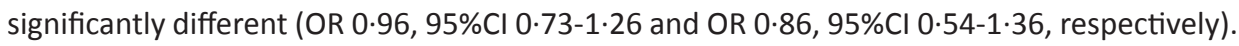
In addition, no significant heterogeneity was found $\left(p=0 \cdot 46, l^{2}=0 \%\right.$ and $p=0 \cdot 35, l^{2}=10 \%$, respectively) (Figure 2). Visual inspection of the funnel plot did not suggest the presence of publication bias (Figure 5B). 
FIGURE 2 | Meta-analysis of 3-year OS (2A) and 5-year OS (2B) and 3-year (2C) and 5-year (2D) DFS SEMS placement as BTS versus emergency surgery for acute malignant colonic obstruction.

A

SEMS as BTS Emergency Surgery

Odds Ratio

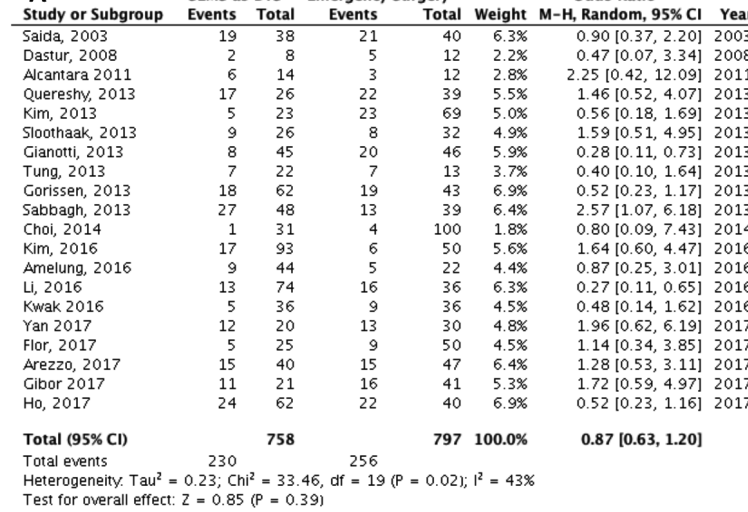

$\mathrm{M}-\mathrm{H}$, Random, $95 \% \mathrm{Cl}$

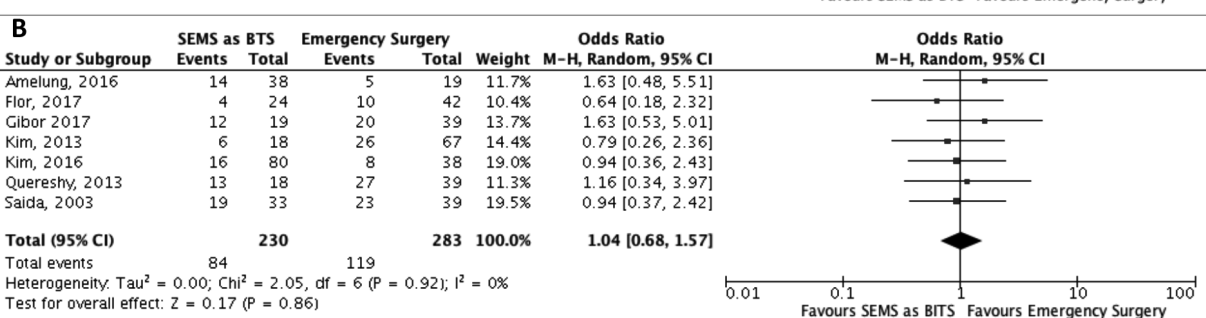

C

SEMS as BTS Emergency Surgery

Odds Ratio

Study or Subgroup Events Total Events Total Weight M-H, Random, $95 \% \mathrm{Cl}$ Year

\begin{tabular}{lrrrrrr}
\hline Gorissen, 2013 & 19 & 60 & 11 & 39 & $10.3 \%$ & $1.18[0.49,2.86] 2013$
\end{tabular}

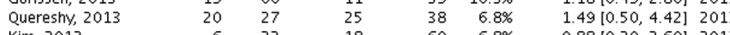

Kim, 2013

Tung, 2013

Sloothaak, 2013

Sabbagh, 2013

Choi, 2014

Kim, 2016

Amelung, 2016

Kwak 2016

$\mathrm{Ho}, 2017$

Arezzo, 2017

Flor, 2017

$\begin{array}{ll}38 & 6.8 \% \\ 60 & 6.8 \%\end{array}$

$0.88[0.29,2.60] 2013$

$0.98[0.19,5.01] 2013$

$1.94[0.68,5.57] 2013$

$1.45[0.62,3.41] 2013$

$0.58[0.24,1.45] 2014$

$1.35[0.60,3.01] 2016$

$1.12[0.33,3.76] 2016$

$0.39[0.09,1.73] 2016$

$0.74[0.31,1.75] 2017$

$1.00[0.39,2.55] 2017$

$0.17[0.04,0.82] 2017$

Total $(95 \% \mathrm{Cl})$

$\begin{array}{lcc}\text { Total events } & 178 & 202 \\ \text { Heterogeneity. } \mathrm{Tau}^{2}=0.00 ; \mathrm{Chi}^{2}=11.76, \mathrm{df}=12(\mathrm{P}=0.46) ; \mathrm{I}^{2}=0 \%\end{array}$

Heterogeneity. $\mathrm{Tau}^{2}=0.00 ; \mathrm{Chi}^{2}=11.76$,

$0.99[0.75,1.31]$

D SEMS as BTS Emergency Surgery Odds Ratio

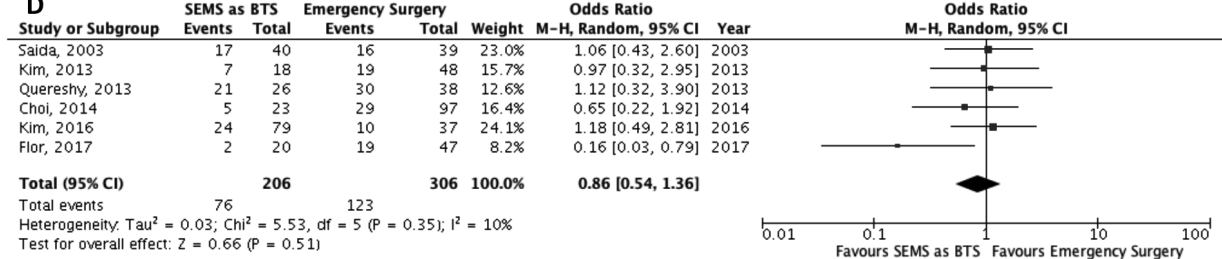


FIGURE 3 | Meta-analysis of overall recurrence (3A) and local recurrence (3B) rate between SEMS placement as BTS versus emergency surgery for acute malignant colonic obstruction.

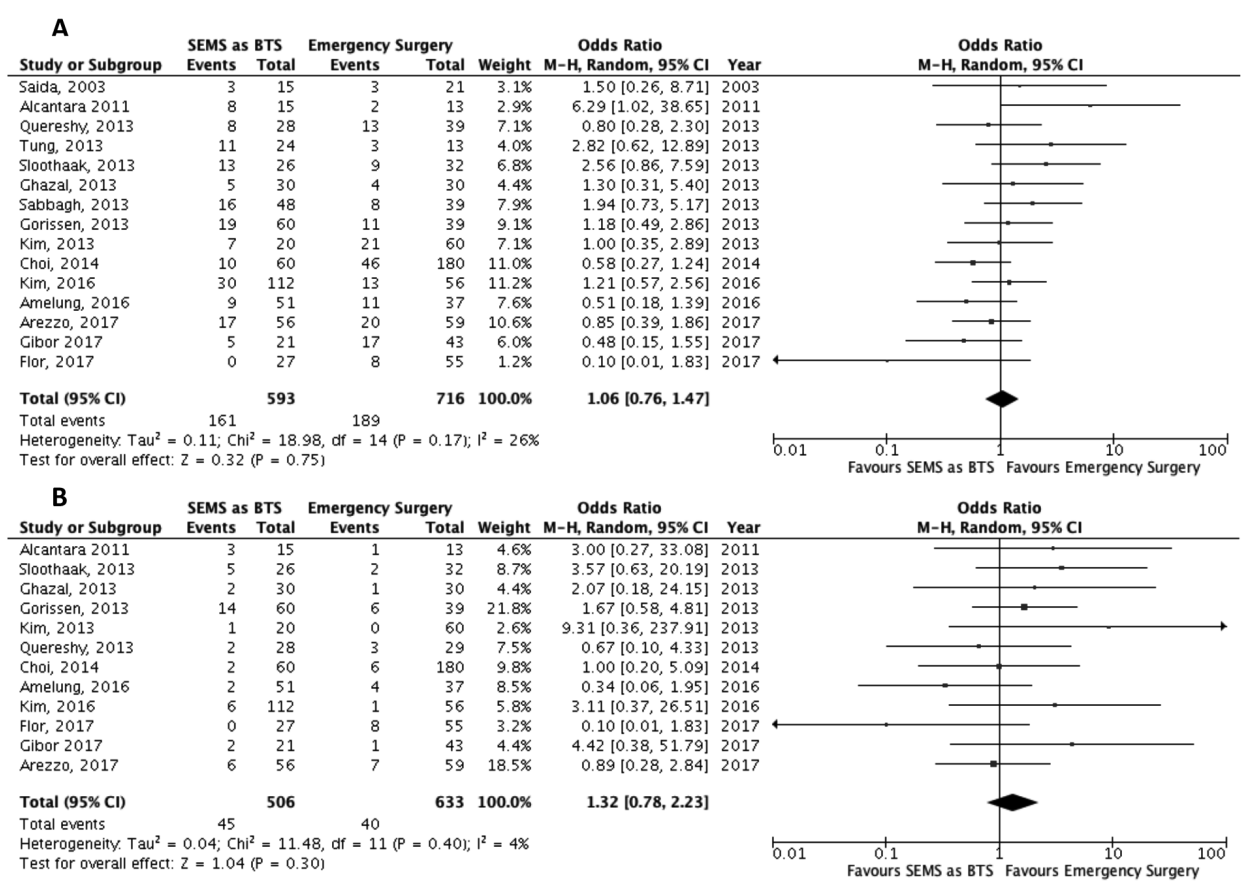

\section{Overall and local recurrence}

A total of fifteen studies reported on recurrence rates, $12,14,17,23,25-28,31-33,36,37,39$ and eleven of these fifteen studies also specifically mentioned whether the recurrence was local or distant. $^{14,17,23,25,27-29,31-33,36,37,39}$ Meta-analysis of any recurrence showed no significant difference between treatment groups with a recurrence rate of $27 \cdot 2 \%$ in the SEMS group versus $26 \cdot 4 \%$ in the ES group (OR 1.06, 95\% Cl 0.76-1.47, 593 vs. 716 patients), with evidence of heterogeneity $\left(p=0 \cdot 17, I^{2}=26 \%\right)$ (Figure $\left.3 A\right)$. Again, the funnel plot did not indicate the presence of publication bias (Figure $5 \mathrm{C}$ ).

For the risk of local recurrence, meta-analysis in a total of 1139 patients ( $506 \mathrm{vs.} 633$ patients) demonstrated no significant difference between treatment groups (OR $1 \cdot 32,95 \% \mathrm{Cl} 0 \cdot 78-2 \cdot 23$ ) (Figure 3B).

\section{Permanent stoma}

Twelve of the twenty-one included studies reported on permanent stoma rates at the end of follow-up. 12,24-28,30,33,35-38 In the SEMS group, $14.7 \%$ (70 out of 475 patients) were found to have a permanent stoma compared to $26 \cdot 5 \%$ (121 out of 456 patients) in the ES group. This difference was statistically significant with an OR of $0.49(95 \% \mathrm{Cl} 0 \cdot 32-0 \cdot 74)$ (Figure $4 \mathrm{~A})$. No significant heterogeneity was identified $\left(p=0 \cdot 19, I^{2}=27 \%\right)$ and there was no evidence of public ation bias (Figure 5D). 
FIGURE 4 | Meta-analysis of permanent stoma rate between SEMS placement as BTS versus emergency surgery for acute malignant colonic obstruction.

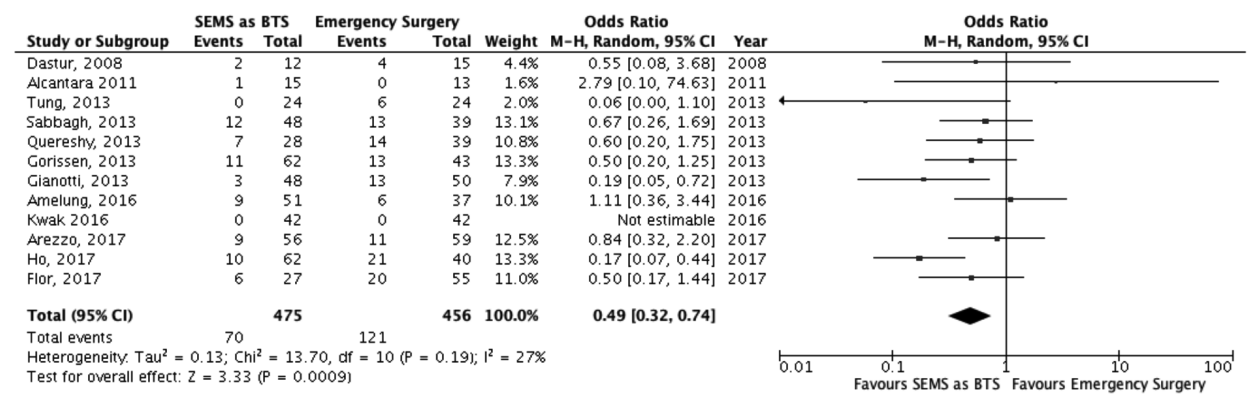

TABLE 3 | Sensitivity analysis of 3-year overall survival between SEMS as BTS and ES for acute malignant colonic obstruction.

\begin{tabular}{|c|c|c|c|c|c|c|c|c|}
\hline \multirow[t]{2}{*}{ Variable } & \multirow[t]{2}{*}{ Subgroup } & \multirow{2}{*}{$\begin{array}{c}\text { No of } \\
\text { studies }\end{array}$} & \multirow{2}{*}{$\begin{array}{l}\text { No of } \\
\text { cases }\end{array}$} & \multirow[t]{2}{*}{ OR } & \multirow[t]{2}{*}{$95 \% \mathrm{Cl}$} & \multirow[t]{2}{*}{ p-value } & \multicolumn{2}{|c|}{ Heterogeneity } \\
\hline & & & & & & & p-value & $1^{2}$ \\
\hline \multirow[t]{2}{*}{ Study Design } & $\mathrm{RCT}$ & 4 & 206 & $1 \cdot 18$ & $0 \cdot 66-2 \cdot 11$ & 0.57 & $0 \cdot 37$ & $4 \%$ \\
\hline & Non-RCT & 16 & 1349 & $0 \cdot 80$ & $0.63-1.03$ & 0.09 & 0.02 & $48 \%$ \\
\hline \multirow[t]{2}{*}{ Region of study } & Europa & 9 & 615 & 0.95 & $0 \cdot 67-1 \cdot 33$ & $0 \cdot 75$ & 0.05 & $48 \%$ \\
\hline & Asia & 11 & 940 & $0 \cdot 79$ & $0 \cdot 58-1 \cdot 07$ & $0 \cdot 13$ & $0 \cdot 07$ & $44 \%$ \\
\hline \multirow[t]{2}{*}{ No of Cases } & $<80$ & 7 & 316 & $1 \cdot 34$ & $0 \cdot 84-2 \cdot 12$ & 0.22 & 0.54 & $0 \%$ \\
\hline & $\geq 80$ & 13 & 1239 & $0 \cdot 74$ & $0.56-0.96$ & $0 \cdot 02 *$ & 0.02 & $49 \%$ \\
\hline \multirow[t]{2}{*}{ No of SEMS cases } & $<40$ & 9 & 483 & $1 \cdot 16$ & $0 \cdot 78-1 \cdot 72$ & 0.47 & 0.53 & $0 \%$ \\
\hline & $\geq 40$ & 11 & 1072 & $0 \cdot 73$ & $0.55-0.97$ & $0 \cdot 03 *$ & 0.01 & $56 \%$ \\
\hline \multirow[t]{2}{*}{ Publication year } & $<2014$ & 10 & 657 & $0 \cdot 85$ & $0 \cdot 62-1 \cdot 18$ & 0.33 & 0.03 & $51 \%$ \\
\hline & $\geq 2014$ & 10 & 898 & 0.86 & $0 \cdot 62-1 \cdot 19$ & 0.36 & 0.08 & $41 \%$ \\
\hline \multirow{2}{*}{$\begin{array}{l}\text { SEMS-related perforation } \\
\text { rate }\end{array}$} & $<8 \%$ & 13 & 898 & $0 \cdot 71$ & $0.52-0.97$ & $0.03 *$ & 0.08 & $38 \%$ \\
\hline & $\geq 8 \%$ & 7 & 657 & 1.07 & $0.76-1 \cdot 50$ & 0.72 & 0.08 & $47 \%$ \\
\hline \multirow{2}{*}{$\begin{array}{l}\text { Technical success rate } \\
\text { of SEMS placement }\end{array}$} & $\geq 90 \%$ & 10 & 839 & 0.68 & $0.50-0.92$ & $0.01 *$ & 0.02 & $53 \%$ \\
\hline & $<90 \%$ & 10 & 725 & 1.15 & $0.81-1.62$ & 0.43 & 0.42 & $2 \%$ \\
\hline
\end{tabular}

\section{Sensitivity analysis}

Sensitivity analysis across six different variables (study design, country, number of patients that received SEMS, technical success rate, publication year and perforation rate) was conducted (Table 3). Sub-analysis for study region (Asia vs. Europe) or publication year (<2014 vs. 22014 ) resulted in similar outcomes in three-year overall survival rate. Sub-analysis of the four randomized studies, showed a non-significant trend towards worse survival in the SEMS group with low heterogeneity (OR $1 \cdot 1895 \% \mathrm{Cl} 0 \cdot 66-2 \cdot 11,102$ vs. 104 patients). ${ }^{17,25,26,37}$ These results are opposite to the non-randomized studies, showing an OR of 0.80 (95\% $\mathrm{Cl}$ 0.63-1.03; 656 vs. 693 patients), with high heterogeneity $\left(I^{2}=48 \%, p=0.02\right)$.

Sub-analysis assessing the potential impact of experience in SEMS procedures revealed a significant survival benefit in the SEMS group in studies in which more than 40 patients 
FIGURE 5 | Funnelplots were plotted of all studies that reported on 3-year overall survival (5A), 3-year disease-free survival (5B), recurrence (5C) and permanent stoma (5D) to identify whether publication bias is likely.

A

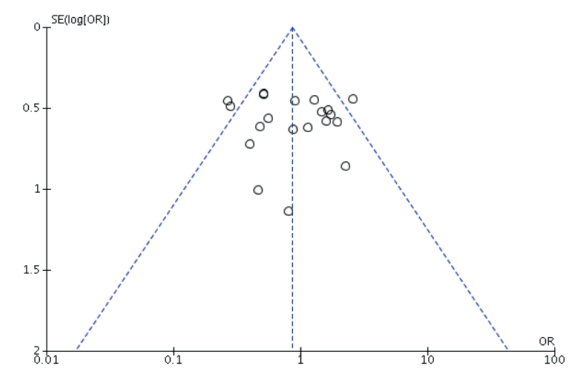

C

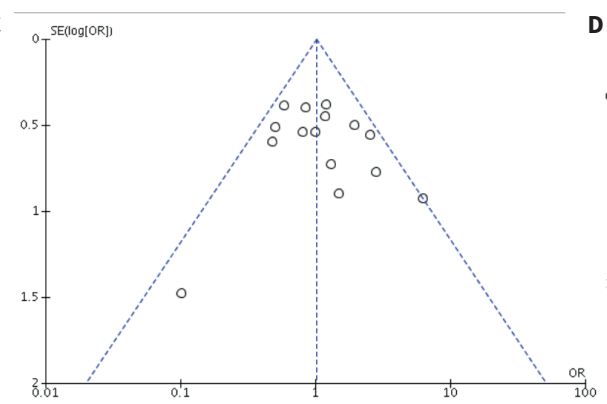

B

D
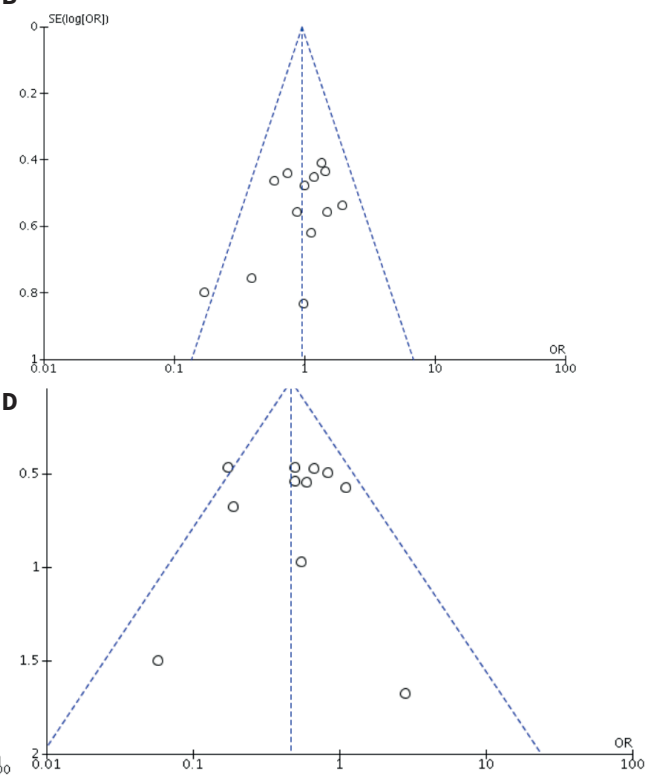

underwent SEMS placement and in studies where the technical success rate was $\geq 90 \%$ (OR $0.73,95 \% \mathrm{Cl} 0.55-0.97$ and $\mathrm{OR0} 0.68,95 \% \mathrm{Cl} 0.50-0.92$, respectively), although with significant heterogeneity $\left(I^{2}=56 \% ; p=0 \cdot 01\right.$ and $\left.53 \% ; p=0.02\right) .12,23,28,30-35,37,38$ Furthermore, studies with a SEMS-related perforation rate below $8 \%$ showed a significantly better survival in the SEMS

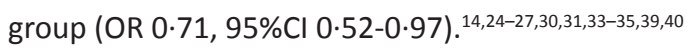

\section{DISCUSSION}

The results of this systematic review and meta-analysis suggest that SEMS placement as BTS in patients with an acute malignant left-sided colonic obstruction might not negatively influence oncological long-term outcomes if compared to emergency surgery. Sensitivity analysis for three-year overall survival even revealed a significant survival benefit after SEMS for studies in which $\mathbf{4 0}$ or more stenting procedures were performed and in studies with a SEMS related perforation rate below $8 \%$. Furthermore, patients treated with SEMS were significantly less likely to end up with a permanent stoma. These findings should, however, still be interpreted with caution, since the majority of patients were included in non-randomized studies, with half of the included studies having a moderate or high risk of bias. Sensitivity analysis showed contradictory trends regarding three-year overall survival between randomized and nonrandomized studies. 
Even though the short-term results of SEMS placement as BTS have been well, ${ }^{5-9}$ stent placement in curative patients has been used with reservation due to uncertainty about its impact on long-term oncological outcomes. This originates from concerns about tumor manipulation during stent insertion, guidewire perforations during stent placement, stent deployment force and eventual micro-perforations at the proximal and distal ends of the stent, which may induce tumor cell dissemination locally, but also in the blood stream. In 2007, Marutchalam et al. showed an increase of cytokeratin 20mRNA expression (a marker for circulating tumor cells) in peripheral venous blood following SEMS placement. ${ }^{13} \mathrm{~A}$ few years later, two studies showed a higher rate of perineural and lymph node invasion after SEMS placement. ${ }^{12,14}$ The prognostic impact of these pathological findings is, however, not clear. A recent multicenter study in patients with stage III colon cancer found no significant association between the presence of circulating tumor cells and worsened survival, although these findings might not be extrapolated to SEMS for obstructing cancers. ${ }^{41}$ Another study focused on perineural invasion in relation to SEMS could not demonstrate an association with tumor recurrence. ${ }^{14}$ These findings underline the need for clinical studies reporting on long-term oncological outcomes, rather than surrogate endpoints.

Three previous meta-analyses, reporting on a total of four, eleven and seventeen studies, respectively, showed no difference in long-term oncological outcomes when SEMS as BTS is compared to emergency surgery. ${ }^{42-44}$ The current meta-analysis includes a total of twenty-one studies and confirms the findings of the previous studies. Although the number of studies increases, there is still a need for more randomized data to be included in meta-analyses on this topic. This is illustrated by the contradictory trends in OR as found in the sensitivity analysis on three-year overall survival, although these were not statistically different. The four RCTs including 206 patients revealed an OR of $1.18(0.66-2 \cdot 11)$ with very low heterogeneity $(4 \%)$, while pooling of the sixteen non-randomized studies including 1349 patients resulted in an OR of $0.80(0.63-1.03)$ with significant heterogeneity (48\%).

Time interval between SEMS and subsequent elective resection, as well as the percentage of SEMS related perforations might be controllable factors that influence oncological outcome. In the study by Gorissen et al., the interval between SEMS and resection was relatively long (19 days). ${ }^{28}$ In 2016, Broholm et al. showed that an increased time from stent placement to elective resection resulted in a significantly higher risk of recurrence (OR 5.1, 95\% $\mathrm{Cl} 1 \cdot 6-5 \cdot 8) .{ }^{45}$ The time interval between stent placement and emergency resection was $<15$ days in all other studies included in this meta-analysis. This is in accordance with the current ESGE guideline that recommends elective resection to be performed within five to seven days following SEMS placement. ${ }^{46}$ Sabbagh et al. (2013) also reported worse overall and disease-free survival after SEMS, despite having a short SEMS-resection time interval of eight days. ${ }^{12}$ In this study, perforation rate was however relatively high. Several studies have indicated that SEMS-related perforation is associated with higher (local) recurrence rates. ${ }^{16,28,30}$ Interestingly, this is underlined by the current study, where sub-analysis of studies with a perforation rate $\geq 8 \%$ showed a trend towards lower survival in the SEMS group. 
SEMS-related perforations might be preventable to some extent. SEMS placement should be avoided in patients treated with bevacizumab, a known risk factor for perforation. ${ }^{18}$ Also, post-stent dilation should also be avoided..$^{18}$ Furthermore, the endoscopists' experience seems to be an important factor for technical and clinical success in stent placement and might be associated with the risk of perforation. ${ }^{47}$ Two studies investigating the learning curve for colorectal stenting recommend at least an experience of 20-30 cases. ${ }^{48,49}$ This is also included in the ESGE guideline recommending a minimum of 20 procedures before SEMS can be placed without supervision. ${ }^{46}$ In the current meta-analysis, such a volume-outcome relationship was confirmed by sub-analysis according to the number of SEMS procedures $(<40 \mathrm{vs.} \geq 40)$.

Nevertheless, potential oncological risks of SEMS might be counterbalanced by some advantages that positively influence oncological outcomes. SEMS facilitates elective laparoscopic resection. Laparoscopic colorectal cancer surgery has been associated with lower mortality risks in population-based studies when compared to open surgery, especially in high risk patients. ${ }^{41,50,51}$ Furthermore, the lower postoperative morbidity rates in patients initially treated with SEMS could result in an earlier start of adjuvant chemotherapy, which has been associated with better survival..$^{52}$ In a shared decision making process, also the advantage of a lower risk of permanent stoma after SEMS should be taken into account, because of its relevance for quality of life..$^{53}$

The current study has several strengths. First, a meta-analysis is an important tool to highlight trends that may not be apparent in individual studies related to statistical power. Secondly, all primary outcomes are objective outcome measures making them less prone to bias. Nonetheless, several limitations should be kept in mind. First, since studies reporting on longterm oncological outcomes following SEMS placement are rare, various methodological designs were included. This meta-analysis was therefore mainly based on non-randomized studies, making it prone to bias. Secondly, surgical techniques differed among the included studies. In most studies, emergency surgery consisted of emergency resection, but in some it entailed the construction of a decompressing stoma. This heterogeneity was expected a priori and therefore a random effects model was used.

Overall, the results of this meta-analysis indicate that SEMS placement is oncologically safe and results in fewer permanent stomas, especially in experienced hands. However, since these results are mainly based on the outcomes of studies with a non-randomised design, these data should be interpreted with caution. More data from randomised trials are needed to draw a definitive conclusion, but currently available evidence should not discourage the use of SEMS as BTS in expert centres to treat patients with left-sided obstructing colon cancer with curative intent. 


\section{REFERENCES}

1. Tanis PJ, Paulino Pereira NR, van Hooft JE, Consten EC, Bemelman WA. Resection of obstructive left-sided colon cancer at a national level: a prospective analysis of short-term outcomes in 1,816 patients. Dig Surg 2015;32:317-324

2. Cheynel N, Cortet M, Lepage C, Benoit L, Faivre J, Bouvier AM. Trends in frequency and management of obstructing colorectal cancers in a well-defined population. Dis Colon Rectum 2007;50:1568-1575

3. Tekkis PP, Kinsman R, Thompson MR, Stamatakis JD. The Association of Coloproctology of Great Britain and Ireland study if large bowel obstruction caused by colorectal cancer. Ann Surg 2004;240;76-81

4. Dohmoto M. New method: endoscopic implantation of rectal stent in palliative treatment of malignant stenosis. Endosc Dig. 1991;3:1507-12

5. Vitale MA, Villotti G, d'Alba L, Frontespezi S, lacopini F, lacopini G. Preoperative colonoscopy after selfexpandable metallic stent placement in patients with acute neoplastic colon obstruction. Gastrointest Endosc. 2006;63:814-9

6. Saida $Y$, Sumiyama $Y$, Nagao J, Takase $M$. Stent endoprothesis for obstructing colorectal cancers. Dis Colon Rectum 1996;39:552-5

7. Arezzo A, Passera R, Lo Secco G, Verra M, Bonino MA, Targarona $\mathrm{E}$, Morino $\mathrm{M}$. Stent as bridge to surgery for left-sided malignant colonic obstruction reduces adverse events and stoma rate compared with emergency surgery: results of a systematic review and meta-analysis of randomized controlled trials. Gastrointest Endosc. 2017; 86:416-426

8. Allievi N, Ceresoli M, Fugazzola P, Montori G, Coccolini F, Ansaloni L. Endoscopic Stenting as Bridge to Surgery versus Emergency Resection for Left-Sided Malignant Colorectal Obstruction: An Updated Meta-Analysis. Int J Surg Oncol. 2017;2017:2863272

9. Atukorale,Y.N. Church,J.L. Hoggan,B.L. Lambert,R.S. Gurgacz,S.L Goodall,S. Maddern,G.J. Self-Expanding Metallic Stents for the Management of Emergency Malignant Large Bowel Obstruction: a Systematic Review. J.Gastrointest.Surg 2016;20;455-462

10. Bridoux,V. Schwarz,L. Kianifard,B. Moutel,G. Herve,C. Tuech,J.J. Systematic review and meta-analysis of randomized clinical trials of self-expanding metallic stents as a bridge to surgery versus emergency surgery for malignant left-sided large bowel obstruction. $\mathrm{Br} J$ Surg 2012;99:469-476
11. Huang X, Lv B, Zhang S, Meng L. Preoperative colonic stents versus emergency surgery for acute left-sided malignant colonic obstruction: a meta-analysis. J Gastrointest Surg 2014;18(3):584-91

12. Sabbagh C, Browet F, Diouf $\mathrm{M}$ et al. Is stenting as "a bridge to surgery" an oncologically safe strategy for the management of acute, left-sided, malignant, colonic obstruction? A comparative study with a propensity score analysis. Ann Surg 2013;258:107-115

13. Maruthachalam K, Lash GE, Shenton BK, Horgan AF. Tumour cell dissemination following endoscopic stent insertion. Br J Surg 2007;94:1151-1154

14. Kim HJ, Choi GS, Park JS et al. Higher rate of perineural invasion in stent-laparoscopic approach in comparison to emergent open resection for obstructing left-sided colon cancer. Int J Colorect Dis 2013;28:207-14

15. Poon J. Pang R. Law W. The impact of colonic stenting on tumor cell dissemination in colorectal cancer patients. Dis.Colon Rectum 2011;54:160

16. Avlund TH, Erichsen R, Ravn S, Ciplys Z, Andersen JC, Laurberg S, Iversen LH. The prognostic impact of bowel perforation following self-expanding metal stent as a bridge to surgery in colorectal cancer obstruction. Surg Endosc 2018;32;328-336

17. Sloothaak DA, van den Berg MW, Dijkgraaf MG, Fockens P, Tanis PJ, van Hooft JE, Bemelman WA. Oncological outcome of malignant colonic obstruction in the Dutch Stent-In 2 trial. Br J Surg 2014;01(13):1751-7

18. van Halsema EE, van Hooft JE, Small AJ et al. Perforation in colorectal stenting: a meta-analysis and a search for risk factors. Gastrointest Endosc 2014;79(6):970-82

19. Moher D, Liberati A, Tetzlaff J, Altman DG, Group P. Prefreed reporting items for systematic reviews and meta-analyses: the PRISMA statement. $B M$ 2009;339;b2535

20. Higgins Julian P T, Altman Douglas G, Gøtzsche Peter C et al. The Cochrane Collaboration's tool for assessing risk of bias in randomised trials BMJ 2011;343;d5928

21. Sterne JA, Hernán MA, Reeves BC, Savović $J$ et al ROBINS-I: a tool for assessing risk of bias in nonrandomised studies of interventions. BMJ 2016;12;355:i4919

22. Higgins JP, Thompson SG, Deeks JJ, Altman DG. Measuring in- consistency in meta-analyses. $B M J$ 2003;327; 557-560 
23. Saida Y, Sumiyama Y, Nagao J, Uramatsu M. Long-term prognosis of preoperative "bridge to surgery" expandable metallic stent insertion for obstructive colorectal cancer: Comparison with emergency operation. Dis.Colon Rectum, 2003;46:S44-S49

24. Dastur JK, Forshaw MJ, Modarai B, Solkar MM, Raymond T, Parker MC. Comparison of short-and long-term outcomes following either insertion of self-expanding metallic stents or emergency surgery in malignant large bowel obstruction. Tech.Coloproctol 2008;12;51-55

25. Alcantara M, Serra-Aracil X, Falco J, Mora L, Bombardo J, Navarro S. Prospective, controlled, randomized study of intraoperative colonic lavage versus stent placement in obstructive left-sided colonic cancer. World J Surg 2011;35:1904-10

26. Tung KL, Cheung HY, Ng LW, Chung CC, Li MK. Endolaparoscopic approach versus conventional open surgery in the treatment of obstructing left-sided colon cancer: long-term follow-up of a randomized trial. Asian J Endosc Surg 2013;6:78-81

27. Quereshy FA, Poon JT, Law WL. Long-term outcomes of stenting as a bridge to surgery for acute left-sided malignant colonic obstruction. Gastroenterology, 2013;144:S1118-S1119

28. Gorissen KJ, Tuynman JB, Fryer E et al. Local recurrence after stenting for obstructing left-sided colonic cancer. BJS 2013;100:1805-9

29. Ghazal AH, El-Shazly WG, Bessa SS, El-Riwini MT, Hussein AM. Colonic endolumenal stenting devices and elective surgery versus emergency subtotal/total colectomy in the management of malignant obstructed left colon carcinoma. I Gastrointest Surg. 2013;17(6):1123-9

30. Gianotti L, Tamini N, Nespoli L et al. A prospective evaluation of short-term and long-term results from colonic stenting for palliation or as a bridge to elective operation versus immediate surgery for large-bowel obstruction. Surg Endosc 2013;27:832-42

31. Choi JM, Lee C, Han YM et al. Long-term oncologic outcomes of endoscopic stenting as a bridge to surgery for malignant colonic obstruction: comparison with emergency surgery. Surg.Endosc 2014;28(9);2649-2655

32. Kim MK, Kye BH, Lee IK et al. Outcome of bridge to surgery stenting for obstructive left colon cancer. ANZ J Surg. 2016;87(9):746

33. Amelung FJ, Ter Borg F, Consten EC, Siersema PD, Draaisma WA. Deviating colostomy construction versus stent placement as bridge to surgery for malignant leftsided colonic obstruction. Surg Endosc. 2016;30(12):5345-5355
34. Li ZX, Wu XH, Wu HY et al. Self-expandable metallic stent as a bridge to elective surgery versus emergency surgery for acute malignant colorectal obstruction. Int J Colorectal Dis. 2016;31(3):561-70

35. Kwak MS, Kim WS, Lee JM et al. Does Stenting as a Bridge to Surgery in Left-Sided Colorectal Cancer Obstruction Really Worsen Oncological Outcomes? Dis Colon Rectum 2016;59(8):725-32

36. Flor-Lorente B, Báguena G, Frasson M et al. E. Selfexpanding metallic stent as a bridge to surgery in the treatment of left colon cancer obstruction: Cost-benefit analysis and oncologic results. Cir Esp. 2017;95(3):143151

37. Arezzo A, Balague C, Targarona E et al. Colonic stenting as a bridge to surgery versus emergency surgery for malignant colonic obstruction: results of a multicentre randomised controlled trial (ESCO trial). Surg Endosc. 2017;31(8):3297-3305

38. Ho KM, Chan KM, Kwok SY, Lau P. Colonic self-expanding metal stent (SEMS) as a bridge to surgery in left-sided malignant. Colonic obstruction: an 8-year review. Surg Endosc. 2017;31(5):2255-2262

39. Gibor U, Perry Z, Tirosh D et al. Comparison of the LongTerm Oncological Outcomes of Stent as a Bridge to Surgery and Surgery Alone in Malignant Colonic Obstruction. Isr Med Assoc J. 2017;19(12):736-740

40. Yan FH, Lou Z, Liu XS et al. Long-Term Oncological Outcomes of Endoscopic Stenting as a Bridge to Surgery Versus Emergency Surgery for Malignant Colorectal Obstruction: A Comparative Study. J Laparoendosc Adv Surg Tech A. 2017;27(6):611-617

41. Sotelo MJ, Sastre J, Meastro ML et al. Role of circulating tumor cells as prognostic marker in resected stage III colorectal cancer. Ann Oncol 2015;26;535-541

42. Zhang Y, Shi J, Shi B, Song CY, Xie WF, Chen YX. Selfexpanding metallic stent as a bridge to surgery versus emergency surgery for obstructive colorectal cancer: a meta-analysis. Surg Endosc. 2012;26(1):110-9

43. Matsuda A, Miyashita $M$, Matsumoto $S$ et al. Comparison of long-term outcomes of colonic stent as "bridge to surgery" and emergency surgery for malignant large-bowel obstruction: a meta-analysis. Ann Surg Oncol 2015;22(2):497-504

44. Ceresoli M, Allievi N, Coccolini F et al. Long-term oncologic outcomes of stent as a bridge to surgery versus emergency surgery in malignant left side colonic obstructions: a meta-analysis. J Gastrointest Oncol 2017;8(5):867-876 
45. Broholm M, Kobborg M, Frostberg E, Jeppesen M, Gögenür I. Delay of surgery after stent placement for resectable malignant colorectal obstruction is associated with higher risk of recurrence. Int $J$ Colorectal Dis. 2017;32(4):513-516

46. van Hooft JE, van Halsema EE, Vanbiervliet $\mathrm{G}$ et al. European Society of Gastrointestinal Endoscopy. Selfexpandable metal stents for obstructing colonic and extracolonic cancer: European Society of Gastrointestinal Endoscopy (ESGE) Clinical Guideline. Endoscopy. 2014;46(11):990-1053

47. Small AJ, Coelho-Prabhu N, Baron TH. Endoscopic placement of self-expandable metal stents for malignant colonic obstruction: long-term outcomes and complication factors. Gastrointest Endosc 2010;71(3):560-572

48. Lee HJ, Park SJ, Cheon JH, Kim TI, Kim WH, Hong SP. What is the necessity of endoscopist for successful endoscopic stenting in patients with malignant colorectal obstruction? Int I Colorectal Dis. 2015;30(1):119-25
49. Williams D, Law R, Pullyblank AM. Colorectal stenting in malignant large bowel obstruction: the learning curve. Int.J.Surg.Oncol 2011;2011;917848

50. Gietelink L, Wouters MW, Bemelman WA, Dekker JW, Tollenaar RA, Tanis PJ; Dutch Surgical Colorectal Cancer Audit Group. Reduced 30-Day Mortality After Laparoscopic Colorectal Cancer Surgery: A Population Based Study From the Dutch Surgical Colorectal Audit (DSCA). Ann Surg. 2016;264(1):135-40

51. Lacy AM, Delgado S, Castells A, Prins HA, Arroyo V, Ibarzabal A, Pique JM. The long-term results of a randomized clinical trial of laparoscopy-assisted versus open surgery for colon cancer. Ann Surg 2008;248;1-7

52. Biaggi JJ, Raphael MJ, Mackillop WJ, Kong W, King WD, Booth CM. Association between time to initiation of adjuvant chemotherapy and survival in colorectal cancer: a systematic review and meta-analysis. JAMA 2011;305:2335-42

53. Young CJ, De-Loyde KJ, Young JM et al. Improving Quality of Life for People with Incurable Large-Bowe Obstruction: Randomized Control Trial of Colonic Stent Insertion. Dis Colon Rectum 2015;58(9):838-4 


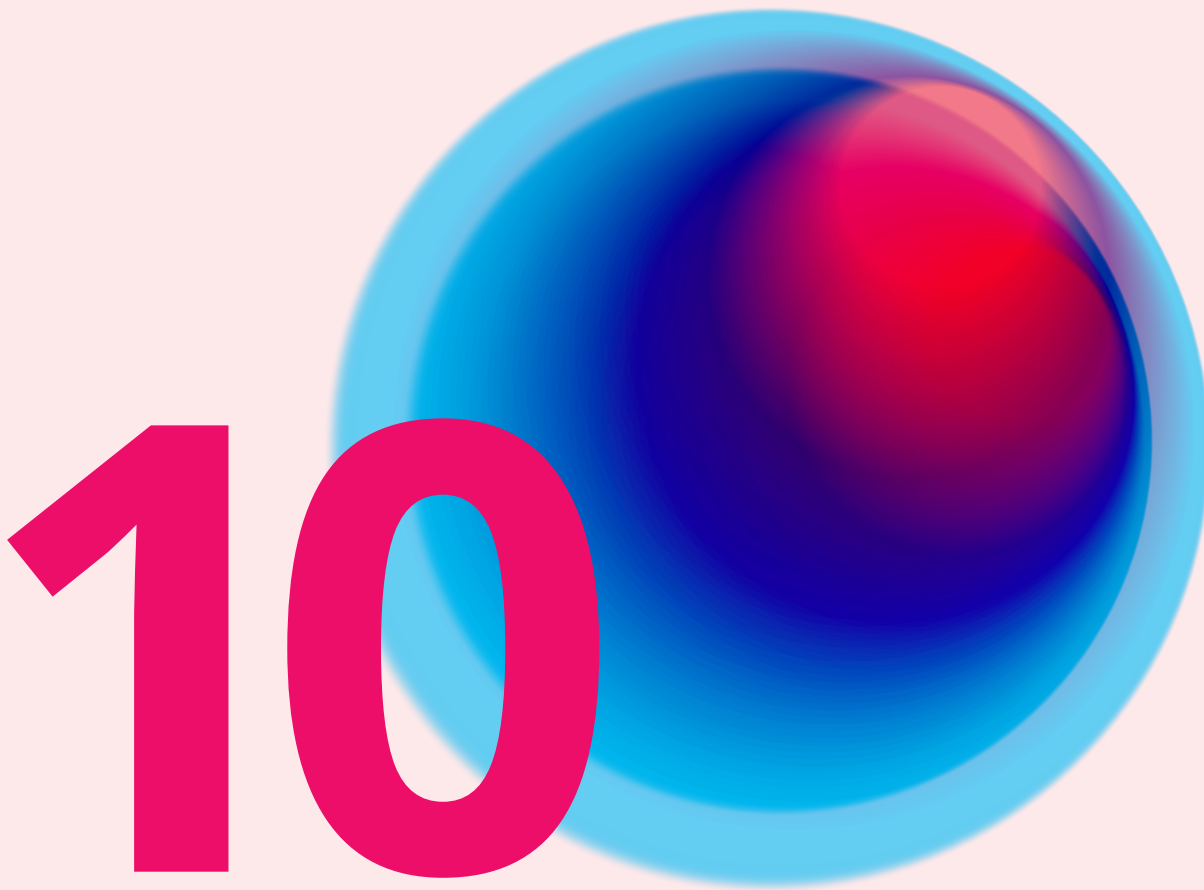

\title{
A propensity score matched analysis of oncological outcomes between stent as bridge to surgery and emergency resection for left-sided obstructive colon cancer
}

Submitted

\author{
F.J. Amelung \\ W.A.A. Borstlap \\ E.C.J. Consten \\ J.V. Veld \\ E.E. van Halsema \\ J. van Hooft \\ W.A. Bemelman \\ P.D. Siersema \\ F. ter Borg \\ P.J. Tanis
}

On behalf of the Dutch Snapshot Research Group 


\section{ABSTRACT}

\section{Introduction}

To investigate the oncological safety of self-expandable metal stent (SEMS) placement as bridge to surgery (BTS) in patients with left-sided obstructive colon cancer in comparison to emergency resection.

\section{Patients and Methods}

A national collaborative research project was conducted to collect long-term outcomes of all patients who underwent resection for left-sided obstructive colon cancer between 2009-2016 in 75 Dutch hospitals. SEMS as BTS was compared with emergency resection in the curative setting after one-to-two propensity score matching.

\section{Results}

Of 2242 potentially eligible patients, 229 underwent SEMS as BTS, of whom 222 were matched with 444 emergency resection patients. Eight SEMS patients underwent emergency resection due to SEMS-related perforation. Including silent and micro-perforations, the overall SEMSrelated perforation rate was $7 \cdot 7 \%$ (17/222). Median follow-up was 36 months (i.q.r. 16-60). Three-year locoregional recurrence rates after SEMS and emergency resection were $11.4 \%$ and $13.6 \%(p=0.457)$, disease-free survival was $58.8 \%$ and $52.6 \%(p=0.175)$ and overall survival was $74.0 \%$ and $68.3 \%(p=0 \cdot 231)$, respectively. SEMS resulted in significantly fewer permanent stomas (23.9\% vs. $43.5 \%$; $p<0.001)$, especially in elderly patients $(29.0 \%$ vs. $57.0 \%$; $p<0.001)$. For SEMS patients with or without perforation, three-year locoregional recurrence rate was $17.9 \%$ and $11.0 \%(p=0.674)$, disease-free survival $48.5 \%$ and $59.6 \%(p=0.717)$ and overall survival $61 \cdot 1 \%$ and $75 \cdot 1 \%(p=0 \cdot 529)$, respectively.

\section{Conclusion}

Overall, SEMS as BTS appears to be an oncological safe alternative for emergency resection with fewer permanent stomas. Nevertheless, the risk of SEMS-related perforation, as well as the risk of a permanent stoma, might influence shared decision making in individual patients. 


\section{INTRODUCTION}

Of all patients with colon cancer, nine to thirteen per cent initially present with an acute obstruction, which accounts for $85 \%$ of colonic emergencies. ${ }^{1}$ Traditionally, left-sided obstructing colon cancer (LSOCC) has been managed with emergency resection. ${ }^{2}$ However, since patients presenting with LSOCC are often elderly and frequently have a deteriorated clinical condition, emergency resection has been associated with substantial morbidity and mortality rates. ${ }^{1-3}$ In addition, many patients end up having a permanent stoma, which is known to have a negative impact on quality of life and independence. ${ }^{4}$

In the past decade, self-expandable metal stent (SEMS) placement has been proposed as an alternative to emergency resection for LSOCC. Initial decompression of the distended colon by SEMS placement can transform the resection into an elective procedure, enabling a laparoscopic approach in a clinically optimized patient. Meta-analyses confirmed higher proportions of laparoscopic surgery after SEMS, with lower postoperative morbidity rates, fewer temporary stoma constructions and, higher primary anastomosis rates. ${ }^{5}$

Even though the short-term benefit has been established in the past years, SEMS as bridge to surgery (BTS) for curative treatment of LSOCC has been debated and is currently not recommended as a standard treatment according to international guidelines. ${ }^{6}$ Concerns include a higher rate of perineural invasion and an increase in tumour cell dissemination after stent insertion. ${ }^{7,8}$ Furthermore, stent- or guidewire-related perforations may increase the risk of recurrence. ${ }^{9}$ SEMS as BTS has also been associated with worse survival although not confirmed by recent meta-analyses. ${ }^{10,11}$

Treatment of LSOCC in the Netherlands has been strongly influenced by two multicentre Dutch randomized trials, the Stent-In 1 and 2 trials. ${ }^{12,13}$ After early termination of both trials due to increased morbidity in the stent arms, SEMS were infrequently used with only a few centres that continued to perform stenting. Emergency resection is therefore still the preferred approach in the vast majority of Dutch hospitals. Allocation of treatment in the Netherlands is therefore mainly hospital-based instead of patient-based. With this propensity score matched population-based analysis, we aimed to provide real-world evidence on long-term oncological outcomes after SEMS as BTS and emergency resection for LSOCC. $^{14}$

\section{METHODS}

\section{Study Design}

A retrospective national collaborative research project was conducted in the Netherlands. The methodology of such a project has been described previously in the first publication of the Dutch Snapshot Research Group (DSRG). ${ }^{15}$ Briefly, short-term data of all patients in the Netherlands undergoing resection of primary colorectal cancer are prospectively collected in the Dutch Colorectal Audit (DCRA, formerly known as DSCA). All patients with a registered resection for LSOCC between 2009 and 2016 were identified from the DCRA database. Short- 
term DCRA data were extended with additional procedural data and long-term surgical and oncological data. For collection of these additional data, a web-based tool was developed and secured according to privacy regulations by Medical Research Data Management (MRDM, Deventer, the Netherlands).

All hospitals $(\mathrm{N}=77)$ registering in the DCRA were invited to participate in this project. Collaborators in each participating hospital were provided with their own registered patients. Under the supervision of one or two consultants, surgical residents performed web-based data collection during a four-month period (August 2017 to December 2017). Following this period, the collected data was analyzed for discrepancies and missing values. Any discrepancies were sent back to the participants, who were asked to verify or complete the data. The combined set of DCRA and DSRG data was anonymized and sent to the central research coordinator. The design of the study and the preparation of the manuscript was performed according to The Strengthening the Reporting of Observational Studies in Epidemiology (STROBE) statement. ${ }^{16}$

\section{Patient selection}

Patients with LSOCC were selected from the DCRA database (2009-2016) using primary tumor localization (splenic flexure, descending colon or sigmoid) and either registration of clinical obstruction and/or an intervention (i.e. SEMS) preceding primary tumor resection. Patients who presented with perforation and those who underwent pre-operative radiotherapy were excluded.

The DSRG collaborators reviewed the original patient files on eligibility criteria with additional variables on signs of clinical obstruction for more precise patient selection. Patients were considered to have an acute colonic obstruction when they had 1) at least one clinical sign of colonic obstruction (bloated abdomen, nausea and/or vomiting) and, 2) radiological signs of colonic obstruction on computed tomography (CT) (dilated large and/or small bowel).

For this specific analysis, only patients who underwent either SEMS as BTS or emergency resection with curative intent were extracted. Whether a patient was treated with curative intent was one of the additionally acquired variables in the DSRG dataset.

\section{Endpoints}

The primary endpoints were three-year disease free and overall survival. Secondary outcomes were locoregional recurrence and permanent stoma rate. Locoregional recurrence was defined as a recurrence at the level of the anastomosis, in a locoregional lymph node or a peritoneal metastasis.

\section{Adjuvant chemotherapy and follow-up}

The Dutch colorectal cancer guideline recommended adjuvant chemotherapy in high-risk stage II (T4, <ten lymph nodes harvested, presentation with obstruction, vascular invasion, undifferentiated tumour) and, stage III patients. Adjuvant chemotherapy until 2016 consisted of either FOLFOX (oxalipatin, leucovorin, 5-fluorouracil) or CAPOX (capecitabine, oxaliplatin) for a total period of 6 months. ${ }^{17}$ 
Follow-up according to the guideline includes clinical visits at 6-month intervals for the first 2-3 years and on a yearly basis thereafter until five years after the curative resection, with at least CEA measurement at each visit. ${ }^{20}$ Abdominal (liver) ultrasonography or a CT is recommended every six months for one to two years following resection and yearly thereafter. In addition, a surveillance colonoscopy is performed between one and three years following resection, with three to five year intervals thereafter.

\section{Statistical analysis}

Since patients were not randomly assigned to a treatment approach, a propensity score method was used to balance baseline covariates between groups. Covariates were selected a priori and included age, sex, ASA-score, BMI, pathological T, N and M stage, location of the tumour, year of presentation, length of the tumour on CT and, previous abdominal surgery. One-to-two nearest neighbour matching without replacement was performed within a calliper of 0.2 logit of the standard deviation of the propensity score.${ }^{18}$ Covariate balance of the matched cohort was assessed using mean standardized differences, with differences less than ten percent indicating good balance. In order to account for the matched nature of the propensity score matched sample, the outcome variables were assessed using a conditional logistic regression.

Before matching, categorical or dichotomous variables were presented as absolute numbers with percentages and compared using the Chi-square test. After matching the McNemar test was used. Continuous variables were reported as mean with standard deviation (SD) or median with interquartile range (i.q.r.) in accordance to their distribution. Normality was assessed by visual inspection of histograms and tested using the Kolmogorov-Smirnov test. According to the distribution, treatment groups were compared using either the independent Students' t-test or the Mann Whitney U-test. Following matching, a paired t-test was used. All analyses were conducted based on an intention-to-treat principle (i.e. emergency resection for SEMS perforation was analysed in the SEMS group).

Comparison of survival probabilities in the matched cohort were performed using a Cox proportional hazards model with shared frailty. Sub-analyses were conducted to compare outcome parameters between SEMS-patients with and without a stent- or guidewire-related perforation. Survival curves in the SEMS group were plotted according to the Kaplan Meier method and tested for significant differences using the logrank test. A p-value $<0.05$ was considered significant. All analyses were performed with IBM SPSS statistics, version 25.0. (IBM Corp Amonk, NY, United States) and R statistical software version R3.3.2 (Matching and Frailtypack package, R Foundation for Statistical Computing, Vienna, Austria).

\section{Ethics}

The medical ethical committee of the Academic Medical Centre in Amsterdam reviewed and approved the observational study design and concluded that informed consent was not required as there was no additional burden for the patient due to the observational design of the study. 


\section{RESULTS}

\section{Patient selection and matching}

Seventy-five of 77 invited Dutch hospitals participated in this collaborative research project. A total of 3879 patients were registered out of the 4216 identified patients (92\%). After retrospective evaluation of original patient files, 726 patients were excluded (Figure 1), of whom 670 patients appeared to be incorrectly registered as obstruction in the DCRA (i.e. stenosing tumour, unable to pass with the endoscope, but without clinical signs of obstruction). This resulted in an overall cohort of 3153 patients with LSOCC. Applying eligibility criteria for this specific analysis resulted in 2242 patients, of whom 229 underwent SEMS as BTS and 2013 emergency resection. Using one-to-two propensity score matching, 222 SEMS patients were matched to 444 patients out of the emergency resection group. Seven SEMS patients were excluded since no compatible matches in the emergency resection group could be found.

Before matching, baseline characteristics were biased for ASA-score, previous abdominal surgery, tumour location and, length of the tumour on CT (mean standardised difference $>10 \%$; Table 1). After propensity score matching, none of these differences remained. The histograms of the estimated propensity scores before and after matching are shown in Figure 2A. The accompanying Nagelkerke $\mathrm{R}^{2}$ of 0.095 indicates a good overlap in propensity scores between treatment groups. The degree of overlay in propensity score (Figure 2B), as well as all standardised mean differences between baseline variables being below ten per cent, indicate that matching was successful in obtaining two well-balanced groups.

\section{Treatment characteristics}

Of all SEMS procedures, $58 \cdot 1 \%$ ( $n=129$ ) were performed in five out of 75 participating hospitals. Overall technical success rate of SEMS was $87 \cdot 4 \%$ (194 of 222 patients). The two main reasons for technical failure were not being able to pass the obstruction with the guide wire $(N=14)$ or stent $(\mathrm{N}=4)$. Due to technical SEMS-failure, twenty-two patients underwent emergency resection within 24 hours, three patients had a decompressing stoma constructed, and, a second attempt of SEMS placement was successful in the three remaining patients.

Two guidewire perforations and six clinically overt SEMS-related perforations occurred, and all these patients underwent emergency resection. During elective resection, two silent SEMS perforations were noted. In addition, the pathologist reported micro-perforation by SEMS in seven other patients. This results in an overall perforation rate of $7 \cdot 7 \%$ (17 out of 222 ).

Table 2 summarizes treatment characteristics of the two groups. Patients in the SEMS group more often underwent laparoscopic resection $(42.5$ vs. $7.0 \%, p<0.001)$ or had a primary anastomosis constructed ( $74.3 \%$ vs. $39.9 \%, p<0.001$ ), and were less likely to have a temporary stoma ( $28.4 \%$ vs. $64 \cdot 1 \%, \mathrm{p}<0.001)$. Thirty- and ninety-day mortality rate, morbidity rate and, the proportion of patients who underwent adjuvant chemotherapy was not different between the treatment groups. 
FIGURE 1| Flowchart of patient selection

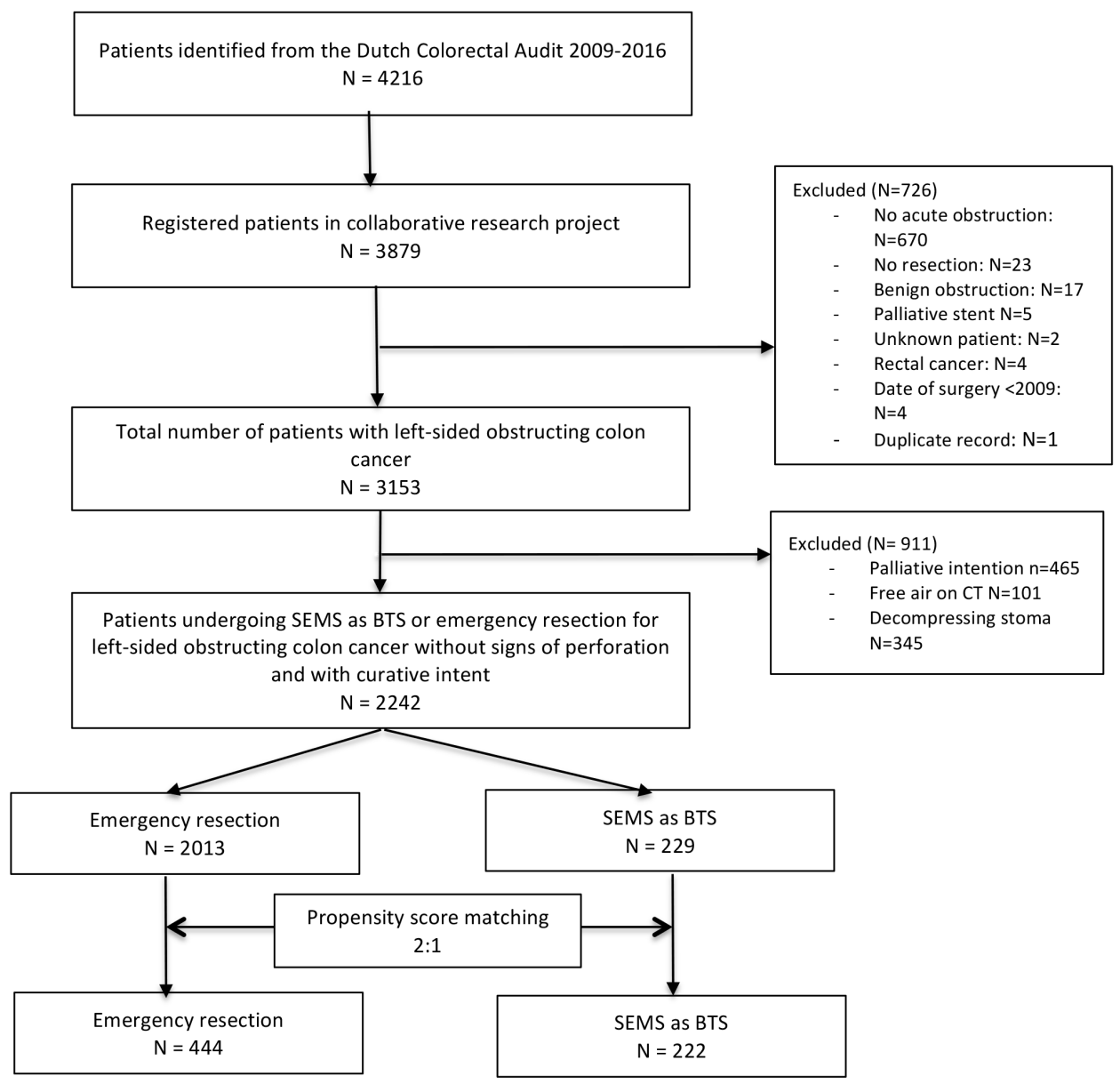

\section{Long-term outcomes}

Median follow-up time in the emergency resection and SEMS groups was 34 (i.q.r. 15-58) and 42 months (i.q.r. 17-65), respectively. Three-year disease free survival was $52 \cdot 6 \%$ and $58 \cdot 8 \%$ and three-year overall survival was $68.3 \%$ and $74.0 \%$ for the emergency resection and SEMS group, respectively. Cox proportional hazard survival analysis with shared frailty showed no significant difference in disease free and overall survival between treatment groups $(p=0.175$ and $p=0 \cdot 231$, respectively (Figure 3 )). An additional Cox-regression analysis was conducted on the unmatched dataset $(\mathrm{N}=2242)$, with propensity score as a covariate. This analysis showed similar outcomes, with no significant difference in disease free and overall survival between treatment groups (HR $1.5(95 \% \mathrm{Cl} 0 \cdot 61-3.76)$ and $\mathrm{HR} 1.8(95 \% \mathrm{Cl} 0 \cdot 64-5 \cdot 21)$, respectively). 
TABLE 1 | Baseline characteristics before and after propensity score matching

\begin{tabular}{|c|c|c|c|c|c|c|}
\hline & \multicolumn{3}{|c|}{ Before PS matching } & \multicolumn{3}{|c|}{ After PS matching 1:2 } \\
\hline & $\begin{array}{c}\text { Emergency } \\
\text { resection } \\
\mathrm{N}=2013(\%)\end{array}$ & $\begin{array}{c}\text { SEMS as } \\
\text { BTS } \\
\mathrm{N}=229(\%) \\
\end{array}$ & MSD & $\begin{array}{c}\text { Emergency } \\
\text { resection } \\
\mathrm{N}=444(\%)\end{array}$ & $\begin{array}{c}\text { SEMS as } \\
\text { BTS } \\
\mathrm{N}=\mathbf{2 2 2}(\%)\end{array}$ & MSD \\
\hline Median age in years (i.q.r.) & $71(62-79)$ & $72(64-80)$ & $6.8 \%$ & $73(63-79)$ & $72(64-80)$ & $1.7 \%$ \\
\hline Male sex & $1071(53.2)$ & $128(55.9)$ & $5.4 \%$ & $253(57.0)$ & $124(55.9)$ & $2.3 \%$ \\
\hline Mean BMI (SD) & $25.5(4.2)$ & $25.7(4.3)$ & $8.3 \%$ & $24.7(4.2)$ & $24.9(4.4)$ & $2.5 \%$ \\
\hline ASA Score & & & $23.3 \%$ & & & $4.7 \%$ \\
\hline ASA 1 & $355(17.7)$ & $49(21.7)$ & & $94(21.4)$ & $48(21.8)$ & \\
\hline ASA 2 & $968(48.2)$ & $120(53.1)$ & & $227(51.6)$ & $117(53.2)$ & \\
\hline ASA 3 & $593(29.5)$ & $53(23.5)$ & & $110(25.0)$ & $51(23.2)$ & \\
\hline ASA 4 & $91(4.5)$ & $4(1.7)$ & & $9(2.0)$ & $4(1.8)$ & \\
\hline pT stage & & & $12.3 \%$ & & & $4.0 \%$ \\
\hline pT1-2 & $78(3.8)$ & $12(5.3)$ & & $26(5.9)$ & $11(5.0)$ & \\
\hline pT3 & $1399(69.9)$ & $162(71.7)$ & & $313(70.7)$ & $158(71.8)$ & \\
\hline pT4 & $530(26.4)$ & $51(22.6)$ & & $104(23.5)$ & $51(23.2)$ & \\
\hline pN stage & & & $6.7 \%$ & & & $3.9 \%$ \\
\hline pNO & $928(46.1)$ & $111(49.1)$ & & $220(49.8)$ & $105(47.9)$ & \\
\hline $\mathrm{pN} 1$ & $699(34.8)$ & $73(32.3)$ & & $140(31.7)$ & $73(33.3)$ & \\
\hline $\mathrm{pN} 2$ & $374(18.6)$ & $41(18.1)$ & & $82(18.6)$ & $41(18.7)$ & \\
\hline Metastases present at presentation & $186(9.3)$ & $24(10.5)$ & $4.2 \%$ & $50(11.3)$ & $22(10.0)$ & $4.5 \%$ \\
\hline Previous abdominal surgery & $595(29.6)$ & $53(23.6)$ & $14.4 \%$ & $112(25.5)$ & $53(24.2)$ & $2.9 \%$ \\
\hline Location of the tumor & & & $23.7 \%$ & & & $1.1 \%$ \\
\hline Sigmoid & 1364 (67.8) & $169(73.8)$ & & $322(72.5)$ & $162(73.0)$ & \\
\hline Descending colon & $375(18.6)$ & 45 (19.7) & & $91(20.5)$ & $45(20.3)$ & \\
\hline Splenic flexure & $274(13.6)$ & $15(6.6)$ & & $31(7.0)$ & $15(6.8)$ & \\
\hline Median length of stenosis in cm (i.q.r.) & $4(3-6)$ & $4(3-5)$ & $23.6 \%$ & $4(3-5)$ & $4(3-5)$ & $6.5 \%$ \\
\hline
\end{tabular}

Abbreviations: ASA-score = Association for Anesthesiology-score; BMI = body mass index; SD = standard deviation; SEMS = self-expandable metallic stent; BTS = bridge to surgery; I.q.r. = inter quartile range; MSD = mean standardized difference; $\mathrm{N}=$ number; $\mathrm{PS}$ = propensity score.

Three-year locoregional recurrence rates were $13.6 \%$ and $11.4 \%(p=0.457)$ in the emergency resection and SEMS group, respectively. Almost twice as many patients in the emergency resection group had a permanent stoma at the end of follow-up compared to the SEMS group (43.5\% vs. $23.9 \%, p<0.001)$. Younger patients (<70 years) were significantly more likely to have their temporary stoma reversed ( 73 out of $135,54.0 \%$ ) compared to elderly patients (30 out of $214,14.0 \%, p<0.001)$. Whether the stoma was reversed was independent of the treatment group. The overall long-term outcomes are summarized in Table 3.

When comparing oncological outcomes after SEMS as BTS with or without guidewire- or SEMSrelated perforation (Figure 4), the three-year disease free survival rates were $48.5 \%$ and $59.6 \%$ $(p=0 \cdot 717)$ and three-year overall survival rates $61 \cdot 1 \%$ and $75 \cdot 1 \%(p=0 \cdot 529)$, respectively. Threeyear locoregional recurrence rates with and without perforation were $17.9 \%$ and $11.0 \%$ $(p=0 \cdot 432)$. 
FIGURE 2 | Overlap in propensity score between treatment groups overall (2A) and after matching (2B)
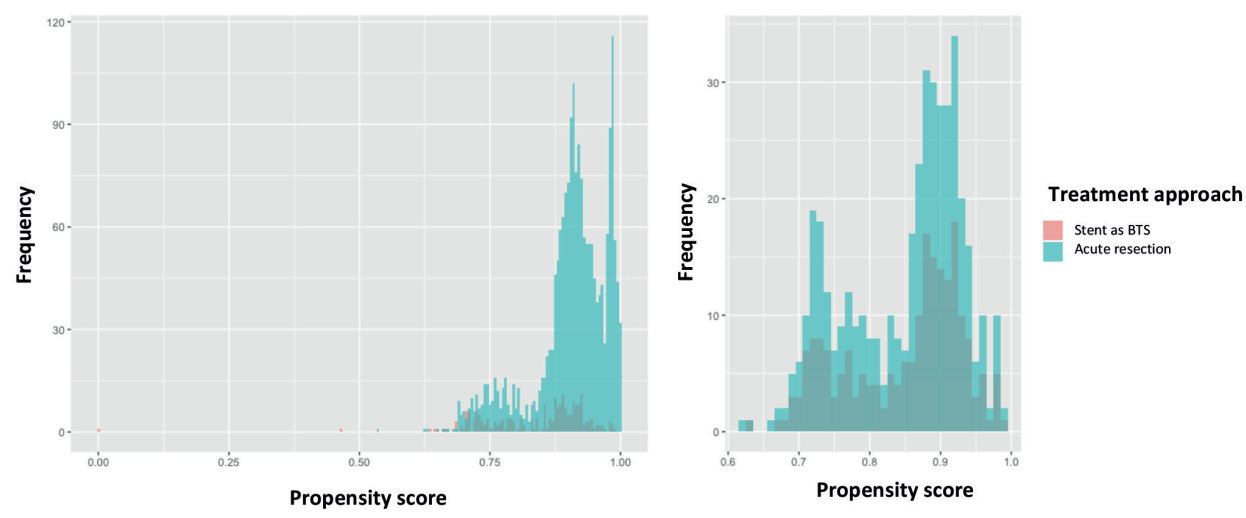

TABLE 2 | Treatment characteristics for the matched cohort; emergency resection versus SEMS placement

\begin{tabular}{|c|c|c|c|}
\hline & $\begin{array}{l}\text { Emergency resection } \\
\mathrm{N}=\mathbf{4 4 4}(\%)\end{array}$ & $\begin{array}{c}\text { SEMS as BTS } \\
\mathrm{N}=\mathbf{2 2 2}(\%)\end{array}$ & P-value \\
\hline Surgical approach & & & 0.001 \\
\hline Open & $410(93.0)$ & $126(57.5)$ & \\
\hline Laparoscopic & $31(7.0)$ & $93(42.5)$ & \\
\hline Resection type & & & 0.095 \\
\hline Sigmoid resection & $279(62.8)$ & $132(59.5)$ & \\
\hline Left hemicolectomy & $99(22.3)$ & $68(30.6)$ & \\
\hline Subtotal colectomy & $28(6.3)$ & $6(2.7)$ & \\
\hline Anterior resection & $11(2.9)$ & $7(3.2)$ & \\
\hline Other & $27(6.1)$ & $9(4.1)$ & \\
\hline Primary anastomosis & $177(39.9)$ & $165(74.3)$ & $<0.001$ \\
\hline Temporary stoma & $286(64.1)$ & $63(28.4)$ & $<0.001$ \\
\hline$<70$ & $114 / 180(63.3)$ & $21 / 91(23.1)$ & $<0.001$ \\
\hline$>70$ & $172 / 256(67.2)$ & $42 / 131(32.1)$ & $<0.001$ \\
\hline Median no. of lymph nodes resected (i.q.r.) & $14(10-19)$ & $16(13-23)$ & $<0.001$ \\
\hline \multicolumn{4}{|l|}{ No of patients with a complication } \\
\hline During the entire treatment approach & $186(41.9)$ & $95(42.8)$ & 0.823 \\
\hline Anastomotic leakage $^{b}$ & $25 / 177(14.1)$ & 18/165 (10.9) & 0.275 \\
\hline Complications requiring re-intervention & $35(7.9)$ & $24(10.8)$ & 0.219 \\
\hline Following tumor resection & $186(41.9)$ & $78(35.1)$ & 0.095 \\
\hline 90-day or in hospital mortality & $28(6.3)$ & $12(5.4)$ & 0.337 \\
\hline Adjuvant chemotherapy & $165(37.2)$ & $84(37.8)$ & 0.868 \\
\hline $\begin{array}{l}\text { Median time in weeks to start of adjuvant } \\
\text { chemotherapy following resection (I.q.r.) }\end{array}$ & $6(5-9)$ & $9(7-12)$ & 0.071 \\
\hline
\end{tabular}

${ }^{a}$ Complications following SEMS placement and/or tumor resection combined. ${ }^{b}$ Not all patients had a primary anastomosis constructed, therefore, the number of patients at risk for an anastomotic leakage differs from the entire treatment group. Abbreviations: SEMS = self-expandable metal stent; BTS = bridge to surgery; I.q.r. = inter quartile range; $\mathrm{N}=$ number. 
TABLE 3 | Long-term outcomes for the matched cohort; emergency resection versus SEMS as BTS

\begin{tabular}{|c|c|c|c|}
\hline & $\begin{array}{c}\text { Emergency resection } \\
\mathrm{N}=\mathbf{4 4 4}(\%)\end{array}$ & $\begin{array}{l}\text { SEMS as BTS } \\
\mathrm{N}=222(\%)\end{array}$ & P-value \\
\hline Median follow-up in months (i.q.r.) & $34(15-58)$ & $42(17-65)$ & 0.002 \\
\hline Permanent stoma at end of follow-up & $193(43.5)$ & $53(23.9)$ & $<0.001$ \\
\hline Age $<70$ & $47 / 180(26.1)$ & $15 / 91(16.5)$ & 0.057 \\
\hline Age $\geq 70$ & $146 / 256(57.0)$ & $38 / 131(29.0)$ & $<0.001$ \\
\hline Any recurrence during follow-up & $225(50.7)$ & $105(47.3)$ & 0.384 \\
\hline Locoregional recurrence & $53(11.9)$ & $22(9.9)$ & 0.516 \\
\hline Anastomotic recurrence & $15(28.3)$ & $7(31.8)$ & \\
\hline Loco-regional lymph node metastasis & $1(1.9)$ & $2(9.1)$ & \\
\hline Peritoneal metastasis & $37(69.8)$ & $13(59.1)$ & \\
\hline Distant metastases & $118(26.6)$ & $48(21.6)$ & 0.125 \\
\hline Para-aortic lymph node metastasis & $3(5.2)$ & $2(8.0)$ & \\
\hline Liver & 73 (61.9) & $32(66.7)$ & \\
\hline Lung & $59(50.0)$ & $19(39.6)$ & \\
\hline Bone & $5(8.5)$ & $4(8.3)$ & \\
\hline Brain & $6(5.1)$ & $3(6.3)$ & \\
\hline 3-year locoregional recurrence rate ${ }^{a}(\%)$ & 13.6 & 11.4 & 0.457 \\
\hline 3-year disease free survivala (\%) & 52.6 & 58.8 & 0.175 \\
\hline 3-year overall survivala $(\%)$ & 68.3 & 74.0 & 0.231 \\
\hline
\end{tabular}

aactuarial probability as determined by Kaplan Meier analysis and tested using shared frailty analysis. Abbreviations: SEMS = self-expandable metal stent; $B T S=$ bridge to surgery; i.q.r. = Inter quartile range; $\mathrm{N}=$ number.

FIGURE 3 | Kaplan Meier curve of disease free survival (3A) and overall survival (3B) when emergency resection and SEMS placement as BTS are compared.

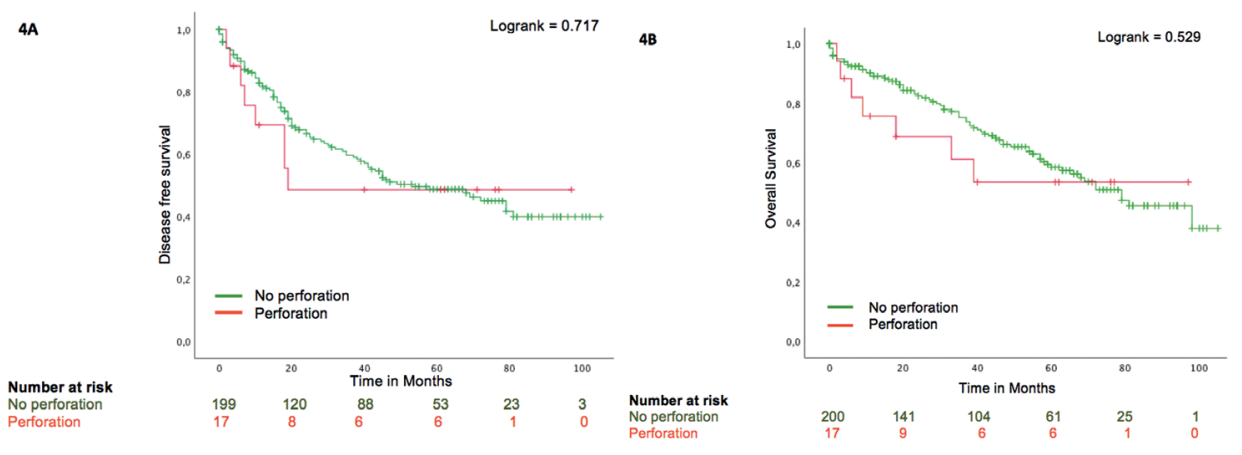


FIGURE 4 | Sub-analysis comparing disease free and overall survival within the SEMS group: perforation versus no perforation (4A/4B).

3A

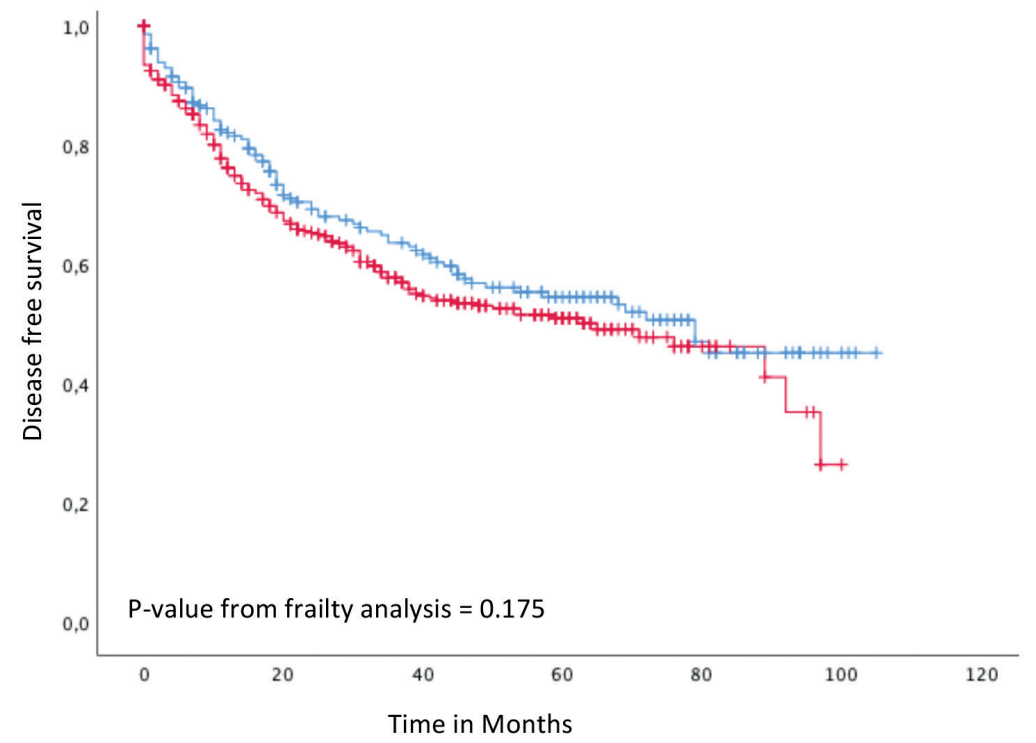

Number at risk

\begin{tabular}{|c|c|c|c|c|c|c|}
\hline Stent & 217 & 127 & 95 & 59 & 24 & 3 \\
\hline Emergency resection & 428 & 244 & 141 & 80 & 16 & 1 \\
\hline
\end{tabular}

3B

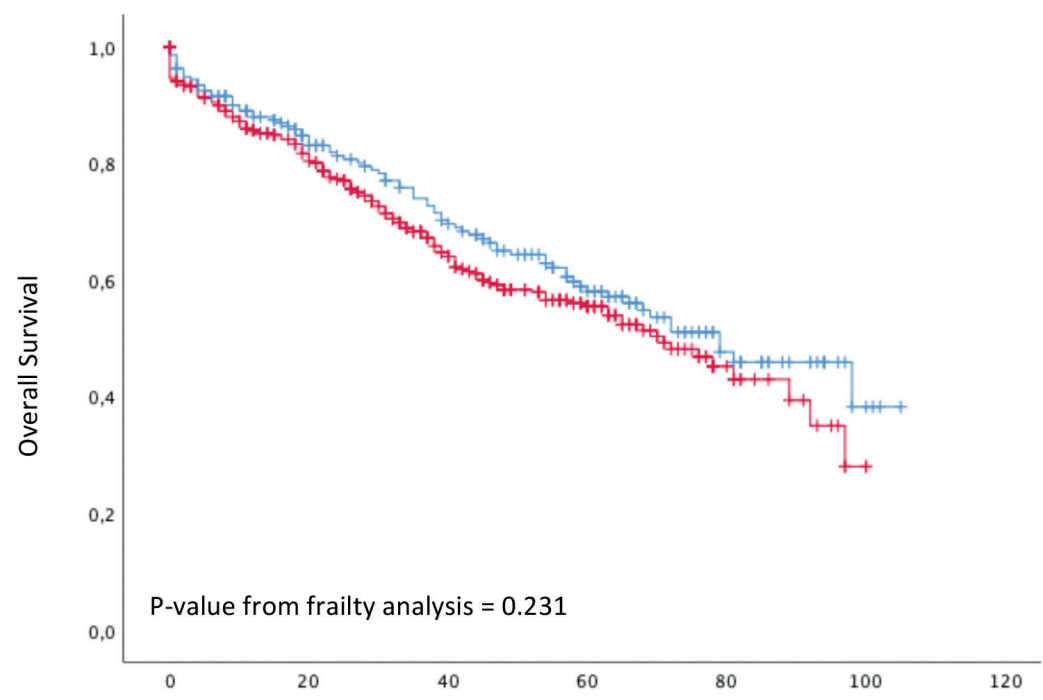

Number at risk

Stent

Time in Months

Emergency resection

428

303

1




\section{DISCUSSION}

The results of this study indicate that SEMS-placement as BTS with curative intent in patients presenting with LSOCC does not negatively influence long-term oncologic outcomes. When compared to emergency resection, no significant differences in locoregional recurrence, and disease free and overall survival rates were found. SEMS-related perforation rate was $8 \%$, with worse oncological outcome parameters in this small subgroup, but not being significantly different from the SEMS patients without perforation. Patients in the SEMS group had significantly fewer permanent stomas at the end of follow-up, especially in elderly patients.

Even though short-term benefit of SEMS placement has been well established, concerns have been raised about worsened oncological outcome. ${ }^{5,7-11}$ Especially SEMS-related perforations have been associated with an increase in loco-regional recurrence and possible worse survival in the past. ${ }^{9,19}$ The results of the current study implicate the same, as a higher recurrence rate and worse survival was seen in the 17 patients $(7 \cdot 7 \%)$ who developed a SEMS-related perforation. Due to insufficient statistical power, however, no firm conclusions could be drawn.

In case of uncomplicated SEMS placement, concerns regarding oncological outcomes have also been raised. Several studies in the past have reported on pathophysiological mechanisms that could possibly lead to worse oncological outcome after SEMS. It was suggested that tumour manipulation and forces acting on the tumour during stent deployment could induce early metastases. These suspicions were strengthened by several pathological studies that reported an increase in circulating tumour cells (CTC) and a higher rate of perineural and lymph node invasion following SEMS placement. ${ }^{7-10}$ These concerns are also reflected in the current guideline of the European Society of Gastro-Enterology (ESGE), which states that SEMS placement should only be first choice in palliative patients or in patients with an increased surgical risk (Age $\geq 70$ and/or ASA-score $\geq 3$ ), and only be performed in centres with substantial experience. ${ }^{6}$

Despite these concerns, the clinical relevance of the previously mentioned surrogate outcome measures remains uncertain. Two recent meta-analyses show an increase in CTCs to be significantly associated with a poor prognosis. ${ }^{20,21}$ Extrapolation of these data to the setting of SEMS as BTS might be inappropriate, since SEMS induced CTCs might not be of similar oncological importance and only a transient phenomenon. Postsurgical CTC increase has often been observed, but studies also show CTC count to normalize or even decrease in the period thereafter. ${ }^{22}$ In addition, Wind et al. have shown CTCs to significantly increase more following open surgery compared to laparoscopic surgery. ${ }^{23}$ As a laparoscopic approach is significantly more often possible in the SEMS group, this could counterbalance the CTC increase initially caused by SEMS placement. Overall, the long-term effects of CTC release by an intervention have not been definitively determined, and conclusions on survival should therefore not be based solely on these surrogate outcome parameters. 
Since publication of the ESGE guideline, multiple studies, including recent meta-analyses, have investigated long-term outcomes following SEMS placement in comparison to emergency resection. ${ }^{10,11}$ They were not able to demonstrate a significant survival difference between treatment groups. Unfortunately, most studies were retrospective and underpowered, seldom had recurrence and survival as the primary outcome measures, and follow up was often relatively short. Only a recent study by Kang et al., comparing 226 SEMS patients to 109 emergency resection patients using propensity score as a covariate, specifically focussed on long-term outcomes.

Even though matching in this cohort was not possible due to a lack of control patients and an insufficient overlap of propensity scores between treatment groups, the methodology of this study is stronger than previous studies. They also found no significant difference in (locoregional) recurrence and survival rates. ${ }^{24}$ Our propensity score matched cohort, including 666 patients of whom 222 were treated with SEMS, further strengthens the previously published conclusions about oncological safety of stenting in LSOCC.

Overall, the $8 \%$ perforation rate and its associated impact on survival, does not seem to impact oncological outcome in the complete SEMS group, since recurrence and survival were similar in both treatment groups. Worse survival due to perforation might therefore be counterbalanced by a positive impact in other SEMS subgroups. For instance, several studies have shown that postoperative complications, occurring non-significantly more in the emergency resection group (Table 2), also negatively affect oncological outcome. ${ }^{25}$ Therefore, different risks and potential advantages related to both strategies should be considered when deciding on optimal management for the individual patient. For example, a young fit patient with low operative risk profile and absence of competing risks of mortality (i.e. cardiovascular) might not want to take the risk of stent perforation. On the other hand, the $8 \%$ perforation risk might outweigh the high operative risk in elderly patients or patients with significant comorbidity. Also, the difference in risk of permanent stoma between age groups should be taken into account, underlining the need for patient-tailored treatment and shared decision-making in patients with LSOCC.

Given its oncological impact, it is crucial to minimize perforation risk. Experience of the endoscopist seems of importance. ${ }^{26}$ In the current study, almost $60 \%$ of the SEMS were placed in dedicated stent-centres, which might explain the relatively low perforation rate. Studies have shown the learning curve for stent placement to be steep, especially when the endoscopist is experienced in colonoscopy and cholangiopancreatography. ${ }^{27}$ Generally, an operator can be labelled 'experienced' after 20 procedures, which is also reflected in the ESGE guidelines. ${ }^{6,28}$ In addition, SEMS-placement is not recommended in patients with a benign stricture or in patients treated with bevacizumab. Also, post-stent dilatation should be avoided. ${ }^{29}$

An important strength of the present study is the high participation of $92 \%$ of the Dutch hospitals. In total, 75 out of the 77 centres contributed to this national cohort study, resulting 
in a good representation of current nationwide practice. In addition, low pre-treatment differences suggested a low risk of selection bias, probably related to the fact that treatment choices were mostly according to institutional protocol, rather than selective allocation to a certain intervention. Furthermore, detailed retrospective review of the original patient files enabled validation and enrichment of the DCRA dataset. Finally, the robustness of our findings is supported by the fact that two different analytical methods resulted in similar outcomes.

Nonetheless, several limitations should be kept in mind. First, only patients who underwent a colonic resection are included in the DCRA registry. Secondly, defining acute colonic obstruction is difficult in the absence of international consensus, and using retrospective chart review. Furthermore, patients who might have died as a consequence of SEMS placement remained out of the scope of the current study. However, since no cases of post-SEMS mortality have been reported in any randomised controlled trial reporting on SEMS as BTS, the influence of this shortcoming is expected to be negligible. ${ }^{5}$ Inherent to real-world evidence, we were also not informed by the degree of guideline adherence regarding follow-up, although there is strong evidence not showing any impact on survival of differences in intensity of follow-up. ${ }^{30}$ Lastly, although propensity score matching was conducted to minimize selection bias, there might still be residual confounding due to unknown factors.

Overall, the data of this propensity score matched population study indicate that SEMS as BTS does not negatively influence long-term oncologic outcomes and significantly reduces the risk of permanent stoma when compared to emergency resection. Therefore, SEMS placement appears to be a valid and safe alternative in dedicated centres. Nevertheless, the risk of SEMSrelated perforation, as well as permanent stoma risk, might influence shared decision making in individual patients with LSOCC. 


\section{REFERENCES}

1. Jullumstro E, Wibe A, Lydersen S, Edna TH. Colon cancer incidence, presentation, treatment and outcomes over 25 years. Colorectal Dis 2011;13(5):512-518

2. Tanis PJ, Paulino Pereira NR, van Hooft JE, Consten EC, Bemelman WA. Resection of obstructive left-sided colon cancer at a national level: a prospective analysis of short-term outcomes in 1,816 patients. Dig Surg 2015;32(5):317-324

3. Tekkis PP, Kinsman R, Thompson MR, Stamatakis JD. The Association of Coloproctology of Great Britain and Ireland study if large bowel obstruction caused by colorectal cancer. Ann Surg 2004;240;76-81

4. Young CJ, De-Loyde KJ, Young JM et al. Improving quality of life for people with incurable large-bowel obstruction: randomized control trial of colonic stent insertion. Dis Colon Rectum 2015;58(9):838-849

5. Arezzo A, Passera R, Lo Secco G, Verra M, Bonino MA, Targarona $\mathrm{E}$, Morino $\mathrm{M}$. Stent as bridge to surgery for left-sided malignant colonic obstruction reduces adverse events and stoma rate compared with emergency surgery: results of a systematic review and meta-analysis of randomized controlled trials. Gastrointest Endosc. 2017;86(3):416-426

6. van Hooft JE, van Halsema EE, Vanbiervliet $\mathrm{G}$ et al. Selfexpandable metal stents for obstructing colonic and extracolonic cancer: European Society of Gastrointestinal Endoscopy (ESGE) Clinical Guideline. Endoscopy. 2014;46(11):990-1053

7. Maruthachalam K, Lash GE, Shenton BK, Horgan AF. Tumour cell dissemination following endoscopic stent insertion. Br J Surg 2007;94:1151-1154

8. Yamashita S, Tanemura M, Sawada G et al K. Impact of endoscopic stent insertion on detection of viable circulating tumor cells from obstructive colorectal cancer. Oncol Lett. 2018;15(1):400-406

9. Sloothaak DA, van den Berg MW, Dijkgraaf MG, Fockens P, Tanis PJ, van Hooft JE, Bemelman WA; collaborative Dutch Stent-In study group. Oncological outcome of malignant colonic obstruction in the Dutch Stent-In 2 trial. Br J Surg. 2014;101(13):1751-7

10. Ceresoli $\mathrm{M}$, Allievi N, Coccolini F et al. Long-term oncologic outcomes of stent as a bridge to surgery versus emergency surgery in malignant left side colonic obstructions: a meta-analysis. J Gastrointest Oncol. 2017;8(5):86-876.

11. Matsuda A, Miyashita M, Matsumoto S, Matsutani T, Sakurazawa N, Takahashi G, Kishi T, Uchida E. Comparison of long-term outcomes of colonic stent as "bridge to surgery" and emergency surgery for malignant large-bowel obstruction: a meta-analysis. Ann Surg Oncol. 2015;22(2):497-504

12. van Hooft JE, Fockens $P$, Marinelli AW, Timmer R, van Berkel AM, Bossuyt PM, Bemelman WA; Dutch Colorectal Stent Group. Early closure of a multicenter randomized clinical trial of endoscopic stenting versus surgery for stage IV left-sided colorectal cancer. Endoscopy. 2008;40(3):184-91

13. van Hooft JE, Bemelman WA, Oldenburg B et al. Colonic stenting versus emergency surgery for acute left-sided malignant colonic obstruction: a multicentre randomised trial. Lancet Oncol. 2011;12(4):344-52

14. Sherman RE, Anderson SA, Dal Pan GJ et al. Real-World Evidence - What Is It and What Can It Tell Us? N Engl J Med. 2016;8;375(23):2293-2297

15. Dutch Snapshot Research Group. Benchmarking recent national practice in rectal cancer treatment with landmark randomised controlled trials. Colorectal Dis. 2017;219-231

16. Von Elm E, Altman DG, Egger M, et al. The Strengthening the Reporting of Observational Studies in Epidemiology (STROBE) statement: guidelines for reporting observational studies. Lancet. 2007 370:1453-1457

17. Integraal Kankercentrum Nederland. National Working Group on Gastrointestinal Cancers. Guideline Colon Cancer 3.0. http://www.oncoline.nl/ colorectaalcarcinoom [accessed April 3rd 2018]

18. Austin, PC. Statistical Criteria for Selecting the Optimal Number of Untreated Subjects Matched to Each Treated Subject When Using Many-to-One Matching on the Propensity Score. Am J Epidemiol. 2010;172(9):10921097

19. Avlund TH, Erichsen R, Ravn S, Ciplys Z, Andersen JC, Laurberg S, Iversen LH.The prognostic impact of bowel perforation following self-expanding metal stent as a bridge to surgery in colorectal cancer obstruction. Surg Endosc. 2018;32(1):328-336

20. Tan $Y$, Wu H.The significant prognostic value of circulating tumor cells in colorectal cancer: A systematic review and meta-analysis. Curr Probl Cancer. 2017;29:S0147-0272(17)30129-0

21. Yang C, Zou K, Zheng L, Xiong B. Prognostic and clinicopathological significance of circulating tumor cells detected by RT-PCR in non-metastatic colorectal cancer: a meta-analysis and systematic review. BMC Cancer. 2017;717-725 
22. Kaifi JT, Li G, Clawson G, Kimchi ET, Staveley-O'Carroll KF. Perioperative circulating tumor cell detection: Current perspectives. Cancer Biology \& Therapy. 2016;17(8):859-869

23. Wind J, Tuynman JB, Tibbe AG, Swennenhuis JF, Richel DJ, van Berge Henegouwen MI, Bemelman WA. Circulating tumour cells during laparoscopic and open surgery for primary colonic cancer in portal and peripheral blood. Eur J Surg Oncol. 2009;35(9):942-50

24. Kang SI, Oh HK, Yoo JS et al. Oncologic outcomes of preoperative stent insertion first versus immediate surgery for obstructing left-sided colorectal cancer. Surg Oncol. 2018;27(2):216-224

25. Ha GW, Kim JH, Lee MR. Oncologic Impact of Anastomotic Leakage Following Colorectal Cancer Surgery: A Systematic Review and Meta-Analysis. Ann Surg Oncol. 2017;24(11):3289-3299

26. Small AJ, Coelho-Prabhu N, Baron TH. Endoscopic placement of self-expandable metal stents for malignant colonic obstruction: long-term outcomes and complication factors. Gastrointest Endosc 2010;71(3):560-572

27. Lee HJ, Park SJ, Cheon JH, Kim TI, Kim WH, Hong SP. What is the necessity of endoscopist for successful endoscopic stenting in patients with malignant colorectal obstruction? Int J Colorectal Dis. 2015;30(1):119-25

28. Williams,D.; Law,R.; Pullyblank,A.M. Colorectal stenting in malignant large bowel obstruction: the learning curve. Int I Surg Oncol., 2011:9:17848

29. van Halsema EE, van Hooft JE, Small AJ et al. Perforation in colorectal stenting: a meta-analysis and a search for risk factors. Gastrointest Endosc 2014;79(6):970-82

30. Jeffery M, Hickey BE, Hider PN, See AM. Follow-up strategies for patients treated for non-metastatic colorectal cancer. Cochrane Database Syst Rev. 2016;24;11:CD002200 



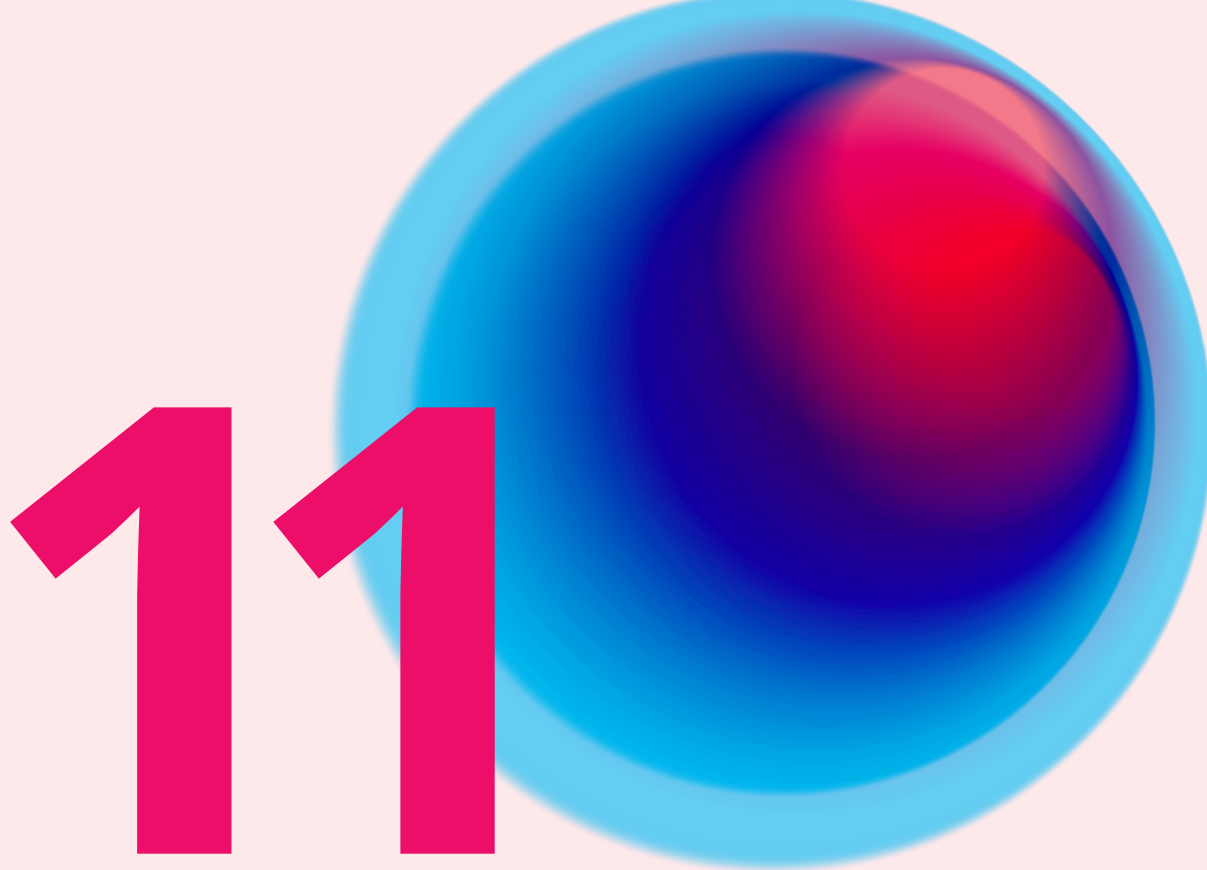

\title{
Incidence and risk factors of ostomy site incisional hernia following stoma reversal
}

British Journal of Surgery Open 2018

\author{
F.J. Amelung \\ L.E.V.M. de Guerre \\ E.C.J. Consten \\ P.M. Verheijen \\ I.A.M.J. Broeders \\ W.A. Draaisma
}




\section{ABSTRACT}

\section{Background}

Stoma reversal is often considered a straightforward procedure with low short-term complication rates. The aim of this study was to determine the incidence of incisional hernia following stoma reversal and identify risk factors for its development.

\section{Methods}

This is an observational study of consecutive patients who underwent stoma reversal between 2009-2015 at a teaching hospital. Patients followed for at least 12 months were eligible. The primary outcome was incisional hernia development at the previous stoma site. Independent risk factors were assessed using multivariable logistic regression.

\section{Results}

Some 318 patients were included. After a median follow-up of 24 months (range 1-89) 110 patients (34.6\%) developed an incisional hernia at the previous stoma site. In 85 (77.3\%) the hernia was symptomatic and 72 patients (65.5\%) underwent surgical correction. A higher BMI (OR: $1.12,95 \% \mathrm{Cl} 1.04-1.21$ ), stoma prolapse (OR: 3.27, 95\% Cl 1.04-10.27), parastomal hernia (OR: $5.08,95 \% \mathrm{Cl} 1.30-19.85$ ) and hypertension (OR: 2.52, 95\% Cl 1.14-5.54) were identified as independent risk factors for incisional hernia development at the previous stoma site. In addition, for patient with underlying malignant disease; having a colostomy compared to an ileostomy increased the odds for incisional hernia development with 5.05 times $(95 \% \mathrm{Cl} 2.28$ 11.23).

\section{Conclusion}

Incisional hernia of the previous stoma site was common and frequently required surgical correction. A higher BMI, reversal of colostomy in patients with an underlying malignancy, stoma prolapse, parastomal hernia and hypertension were identified as independent risk factors. 


\section{INTRODUCTION}

Stoma reversal is often regarded as a straightforward and safe surgical procedure with low short-term postoperative morbidity and mortality rates. ${ }^{1}$ The stoma incision site however is at risk for incisional hernia development. ${ }^{2}$ Early studies have estimated incisional hernia incidence following stoma reversal at approximately $7 \%{ }^{3,4,5}$ Recent studies specifically designed to investigate stoma site herniation have reported a considerably higher incidence of 30 $35 \% .6,7,8$ Incisional hernias following stoma closure may therefore be an underestimated clinical problem. ${ }^{6}$ Incisional hernias can cause abdominal pain, discomfort and impaired quality of life. ${ }^{6}$ Medical consultation is often sought. Approximately $50 \%$ of the patients requires surgical correction to relief symptoms. ${ }^{6}$ Hernia repair is often considered a high-risk procedure due to co-morbidities and intra-abdominal adhesions from previous abdominal surgery. ${ }^{9,10}$ The rate of incisional hernia at the stoma site seems to be much higher than rates of herniation following other abdominal incisions (10-15\%). ${ }^{11,12}$

Further investigation of risk factors is warranted..$^{12}$ This could lead to new strategies for hernia prevention in high-risk patients. Prophylactic mesh placement at the previous stoma site during stoma closure may markedly decrease the risk of incisional hernia development. ${ }^{6,13}$ It does however increase medical costs per procedure and may increase the risk of surgical site infection. ${ }^{14}$ In this study the aim was to accurately quantify the incidence of and identify risk factors for incisional hernia at the previous stoma site following reversal.

\section{METHODS}

This was an observational study involving consecutive patients who underwent stoma reversal between January 2009 and December 2015 at a teaching hospital in the Netherlands. They were identified by operation code in the hospital's electronic medical system. Patients older than 18 years were analysed when they underwent reversal of an ileostomy or colostomy and their follow-up was longer than 12 months or an incisional herniation was observed. Medical records were retrieved to see if incisional hernia was identified by physical examination, computed tomography (CT) or ultrasound during follow-up. Patients whose clinical or radiological follow-up (CT or ultrasound) was less than twelve months following stoma reversal were contacted and asked whether the diagnosis of an incisional hernia was made in another hospital or by their general practitioner. When the diagnosis was considered correct, two additional questions were asked. When was the hernia diagnosed and had they undergone surgical correction. If incisional hernia had not been diagnosed at that time, the patient was invited to the outpatient clinic for physical examination of their previous stoma site. Any palpable defect was recorded. When it was clinically unclear, abdominal ultrasound was performed in supine position during a Valsalva manoeuvre.

All stoma reversals were performed under general anaesthesia. All patients received prophylactic antibiotics (cefmetazole $1 \mathrm{~g}$ and metronidazole $500 \mathrm{mg}$ ). An incision was made around the stoma and the bowel mobilized from surrounding tissues. In patients with a loop 
stoma, intestinal continuity was restored without the need for an additional laparotomy. When patients had an end stoma, laparotomy or laparoscopy was necessary to restore bowel continuity. Anastomosis was created by a double stapling technique for loop stomas and with a circular stapler for end stomas. The fascia defect was closed primarily with either continuous or interrupted absorbable sutures. The skin was closed using a purse-string technique. ${ }^{15}$ All procedures were performed or supervised by, one of four experienced gastro-intestinal surgeons.

Data collected from the patients' medical records included patient characteristics (age at stoma site closure, sex, body mass index, ASA-score, smoking behaviour), comorbidities (diabetes, chronic obstructive pulmonary disease, hypertension (defined as a systolic tension $>140 \mathrm{mmHg}$ on more than three consecutive occasions), cardiovascular disease, previous abdominal surgery), surgical variables (operation technique, material and technique of sutures for fascia closure, preoperative haemoglobin, length of stay during stoma construction and reversal), stoma characteristics (colostomy or ileostomy, loop or end, indication for stoma) complications after reversal (re-intervention, surgical site infection, anastomotic leakage, postoperative ileus), complications while stoma was in situ (parastomal hernia, stoma prolapse, skin irritation, surgical site infection, dehydration, stoma obstruction, high output stoma, pneumonia). Indication for the primary operation (benign or malignant), thirty-day mortality and in-hospital mortality following stoma reversal were documented.

\section{End points}

The primary outcome measure was whether or not an incisional hernia developed at the previous stoma site. Incisional hernia was defined as a defect within the musculature and fascia detected either by physical examination, ultrasound examination or computed tomography. Other outcomes included risk factors for stoma site incisional hernia development identified by multivariable analysis.

\section{Statistical analysis}

Statistical analysis was performed using SPSS version 23.0. Normally distributed continuous variables were expressed as means $( \pm S D)$ and non-normally distributed variables were expressed as medians (range). Categorical data were presented as number and percentages and compared using Fisher's exact test or chi square test as appropriate. Continuous variables were compared using Mann-Whitney $\mathrm{U}$ and Student's T-test as appropriate. Multivariable logistic regression analysis was performed to identify independent predictors of incisional hernia development following stoma reversal. Variables with a $p$-value $<0.10$ in univariable analysis were entered in multivariate logistic regression analysis, keeping a 1:10 event-pervariable (EPV) ratio in mind. Furthermore, several pre-specified theoretically and biologically plausible interaction terms were added to the multivariable analysis to account for possible interaction. Statistical significance was set at $p<0.05$ for all analyses. 


\section{RESULTS}

409 patients underwent reversal of an ileostomy or colostomy between January 2009 and December 2015. 292 patients had been followed for more than 12 months or were diagnosed with a hernia before 12 months. The other 117 patients had limited follow-up. Twenty-five patients attended an outpatient clinic for further examination, so that eventually 318 patients (77.8\%) were analysed (Figure 1).

Baseline characteristics are shown in Table 1. Of the 318 patients, 143 (45.0\%) had a temporary ileostomy and 175 (55.0\%) a temporary colostomy. Ninety-five patients (29.9\%) experienced stoma related complications while the stoma was in situ.

Intraoperative data and complications are shown in Table 2. Median follow-up was 24 months (range 12-89). Since most patients were followed-up after resection of malignancy, most patients ( $n=230,72.3 \%$ ) had radiological follow-up exceeding 12 months. The guidelines for oncologic follow-up in the Netherlands include outpatient visits at a 6-month interval for the first 2-3 years and once a year until 5 years after the resection. A CT or abdominal ultrasound

FIGURE 1 | Flowchart of patients selection

All patients who underwent stoma reversal between 2009 and 2015

$$
\mathrm{N}=409
$$

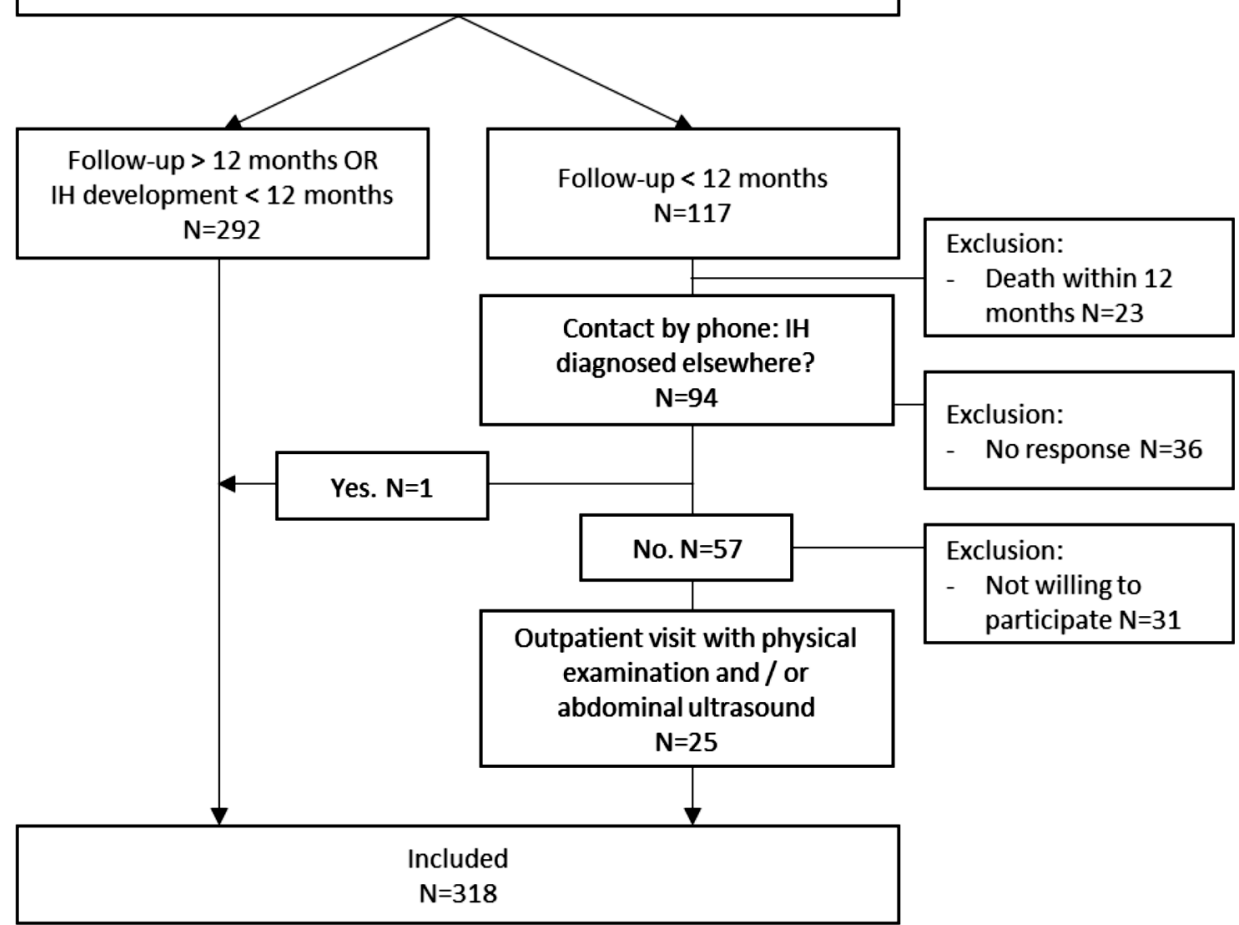


TABLE 1 | Baseline characteristics

\begin{tabular}{|c|c|c|c|c|}
\hline Characteristics & $\begin{array}{c}\text { Total } \\
\mathrm{N}=\mathbf{3 1 8}(\%)\end{array}$ & $\begin{array}{c}\text { Hernia } \\
\mathrm{N}=110(\%)\end{array}$ & $\begin{array}{l}\text { No hernia } \\
\text { N= } 208 \text { (\%) }\end{array}$ & p-value \\
\hline Median age in years (i.q.r.) & $61.7(53-72)$ & $61.7(52-73)$ & $61.7(54-71)$ & .992 \\
\hline Male sex & $170(53.5)$ & $52(47.3)$ & $118(56.7)$ & .108 \\
\hline Median BMI (i.q.r.) & $26(23-30)$ & $27.2(23.5-32.5)$ & $25.5(22-28)$ & $.000^{*}$ \\
\hline ASA score & & & & .700 \\
\hline$|-| \mid$ & 286 (89.9) & $98(89.1)$ & $188(90.4)$ & \\
\hline III-IV & $32(10.1)$ & $12(10.9)$ & $20(9.6)$ & \\
\hline Indication for stoma construction & & & & $.001^{*}$ \\
\hline Protection of anastomosis & $194(61.0)$ & $51(46.4)$ & $143(68.8)$ & \\
\hline Anastomotic leakage & 25 (7.9) & $10(9.1)$ & $15(7.2)$ & \\
\hline Acute colonic obstruction & $52(16.4)$ & $24(21.8)$ & $28(13.5)$ & \\
\hline Other Benign ${ }^{a}$ & $47(14.8)$ & $25(22.7)$ & $22(10.6)$ & \\
\hline Underlying malignant disease & $191(60.1)$ & $48(43.6)$ & $143(68.8)$ & $.000^{*}$ \\
\hline Current smoker & $66(20.7)$ & $24(22.2)$ & $42(20.2)$ & .665 \\
\hline Median pre-operative haemoglobin (i.q.r.) & $7.9(7.2-8.5)$ & $8.1(7.3-8.5)$ & $7.8(7.1-8.4)$ & .140 \\
\hline \multicolumn{5}{|l|}{ Co-morbidity } \\
\hline Hypertension & $106(33.3)$ & $47(42.7)$ & $59(28.4)$ & $.012^{*}$ \\
\hline Diabetes Mellitus & $41(12.9)$ & $15(13.6)$ & $26(12.6)$ & .861 \\
\hline COPD & $22(6.9)$ & $6(5.5)$ & $16(7.7)$ & .643 \\
\hline Cardiovascular disease & $69(21.7)$ & $28(25.5)$ & $41(19.7)$ & .254 \\
\hline Primary abdominal surgery & & & & .796 \\
\hline - Open & $138(43.4)$ & $39(44.5)$ & $89(42.8)$ & \\
\hline - Laparoscopic & $160(50.3)$ & $53(48.2)$ & $107(51.4)$ & \\
\hline$-N A^{b}$ & $20(6.3)$ & $8(7.3)$ & $12(5.8)$ & \\
\hline Primary resection type & & & & .131 \\
\hline - Left hemicolectomy & $22(6.9)$ & $10(9.1)$ & $12(5.8)$ & \\
\hline - Right hemicolectomy & $13(4.1)$ & $4(3.6)$ & $9(4.3)$ & \\
\hline - Subtotal colectomy & 19 (6.0) & $7(6.4)$ & $12(5.8)$ & \\
\hline - Sigmoidectomy & $101(31.8)$ & $43(39.1)$ & $58(27.9)$ & \\
\hline - LAR & $143(45.0)$ & $38(34.5)$ & $105(50.5)$ & \\
\hline$-N A^{b}$ & $20(6.3)$ & $8(7.3)$ & $12(5.8)$ & \\
\hline Adjuvant therapy administration & $49(15.4)$ & $11(10.0)$ & $38(18.3)$ & $.071^{*}$ \\
\hline Emergency surgery & $87(27.4)$ & $39(35.5)$ & $48(23.1)$ & $.024^{*}$ \\
\hline
\end{tabular}

Abbreviations: i.q.r. = Inter quartile range, $\mathrm{BMI}=$ Body Mass Index, ASA = American Society of Anaesthesiologists, COPD = chronic obstructive pulmonary disease, $N A=$ not applicable. ${ }^{*} p$-value $<0.1$, this variable will therefore also be included in the multivariate logistic regression analysis. ${ }^{a}$ Other Benign includes diverticulitis, slow transit colon and anal fistulas. ${ }^{\mathrm{b}} \mathrm{NA}$ not applicable; in these cases stoma construction was the primary surgery without subsequent resection (anal fistula).

is made 6 months after resection and on a yearly basis thereafter. ${ }^{16}$ The remaining 88 patients with a follow-up $<12$ months underwent physical examination to detect incisional hernia. Following stoma reversal 110 patients developed an incisional hernia at the stoma incision site. The median time to hernia detection was 7 months. Within 12 months, 83 (75.5\%) of all reported hernias were detected. In 56 (50.9\%) patients incisional herniation was diagnosed using physical examination, in $12(10.9 \%)$ it was diagnosed by CT and in $8(7.3 \%)$ patients by ultrasound. In 33 (30\%) patients a hernia was diagnosed both during physical examination and on $\mathrm{CT}$. 
TABLE 2 | Intraoperative data and complications

\begin{tabular}{|c|c|c|c|c|}
\hline Characteristics & $\begin{array}{l}\text { Total } \\
\mathrm{N}=318(\%)\end{array}$ & $\begin{array}{l}\text { Hernia } \\
\mathrm{N}=110(\%)\end{array}$ & $\begin{array}{l}\text { No hernia } \\
\mathrm{N}=208(\%)\end{array}$ & p-value \\
\hline Type of stoma & & & & $.001^{*}$ \\
\hline - Ileostomy & $143(45.0)$ & $36(32.7)$ & $107(51.4)$ & \\
\hline - Colostomy & $175(55.0)$ & $74(67.3)$ & $101(48.6)$ & \\
\hline Type of stoma & & & & 1.000 \\
\hline - End stoma & $282(88.7)$ & $98(89.1)$ & $184(88.5)$ & \\
\hline - Loop stoma & $36(11.3)$ & $12(10.9)$ & $24(11.5)$ & \\
\hline Stoma related complications & $100(31.4)$ & $37(33.6)$ & $63(30.3)$ & .612 \\
\hline - Prolapse & $24(7.5)$ & $13(16.3)$ & $11(7.1)$ & $.039 *$ \\
\hline - Parastomal hernia & $17(5.3)$ & $10(12.5)$ & $8(5.1)$ & $.067^{*}$ \\
\hline - Dehydration & $19(8.0)$ & $4(5.0)$ & $15(9.5)$ & .313 \\
\hline - Obstruction & $17(7.2)$ & $5(6.3)$ & $12(7.6)$ & .785 \\
\hline - Necrosis & $5(2.1)$ & $2(2.5)$ & $3(1.9)$ & 1.00 \\
\hline - High output & $23(9.7)$ & $7(8.9)$ & $16(10.1)$ & .820 \\
\hline - Retraction & $4(1.7)$ & $0(0)$ & $4(2.6)$ & .303 \\
\hline Re-admission for stoma related complication & $25(7.9)$ & $10(9.1)$ & $15(7.2)$ & .662 \\
\hline $\begin{array}{l}\text { Median time between stoma construction and } \\
\text { closure in weeks (i.q.r.) }\end{array}$ & $16(12-30)$ & $16(12-31)$ & $16(12-30)$ & .399 \\
\hline Suture material for fascia closure & & & & .759 \\
\hline - Polydioxanone (PDS, Ethicon) & $57(18.0)$ & $21(21.0)$ & 36 (19.6) & \\
\hline - Polyglactin (Vicryl, Ethicon) & $227(71.4)$ & $79(79.0)$ & $148(80.4)$ & \\
\hline Suture technique & & & & .461 \\
\hline - Intermittent & $217(68.2)$ & $73(73.7)$ & $144(78.3)$ & \\
\hline - Continuous & $66(20.8)$ & $26(26.3)$ & $40(21.7)$ & \\
\hline Stoma closure technique & & & & .856 \\
\hline - Local reversal & $257(80.8)$ & $94(85.5)$ & $163(78.4)$ & \\
\hline - Including laparotomy & $45(14.2)$ & $14(12.7)$ & $31(14.9)$ & \\
\hline - Including laparoscopy & $16(5.0)$ & $2(1.8)$ & $14(6.7)$ & \\
\hline \multicolumn{5}{|l|}{ Complications following reversal } \\
\hline - Surgical site infection & $29(9.1)$ & $16(14.7)$ & $13(6.3)$ & $.023^{*}$ \\
\hline - Pneumonia & $17(5.3)$ & $9(8.9)$ & $8(4.0)$ & .112 \\
\hline - Anastomotic leakage & $3(1.0)$ & $1(0.9)$ & $2(1.0)$ & 1.00 \\
\hline - Obstruction & $22(7.0)$ & $9(8.3)$ & $13(6.3)$ & .643 \\
\hline - Mortality & & & & \\
\hline $\begin{array}{l}\text { Complications following reversal } \\
\text { requiring re-intervention }\end{array}$ & $30(9.6)$ & $11(10.1)$ & 19 (9.3) & .842 \\
\hline Median follow-up time (i.q.r.) ${ }^{a}$ & & $24(12-48)$ & $28(18-48)$ & .578 \\
\hline - Radiological follow-up >12 months & $230(72.3)$ & $78(70.1)$ & $152(73.1)$ & .388 \\
\hline
\end{tabular}

Abbreviations: i.q.r. $=$ Inter quartile range. ${ }^{*}$ p-value $<0.1$, this variable will therefore also be included in the multivariate logistic regression analysis. ${ }^{a}$ Median follow-up time: the longest follow-up time was taken into account for this variable, this could be the longest clinical (with physical examination) or radiological (abdominal ultrasound or CT) follow-up time.

The incisional hernia was symptomatic in 85 of 110 patients (75.5\%), of whom 72 (65.5\%) required surgical correction. Patients with symptomatic hernias who chose not to undergo surgical correction either had minor symptoms or were sufficiently helped with non-surgical solutions such as an abdominal wall support. Several variables were identified as possible risk factors for incisional hernia development since their $p$-value was $<0.1$ in univariate analysis. $\mathrm{BMI}$, having a colostomy, stoma prolapse, parastomal hernia, surgical site infection following 
TABLE 3 | Primary multivariable analysis of risk factors for the development of an incisional hernia

\begin{tabular}{|c|c|c|}
\hline Variable & OR $(95 \% \mathrm{Cl})$ & P-value \\
\hline BMI & $1.12(1.04-1.21)$ & $.004^{*}$ \\
\hline Adjuvant therapy & & .457 \\
\hline Yes & $.63(.18-2.15)$ & \\
\hline No & 1.00 (Ref) & \\
\hline \multicolumn{3}{|l|}{ Stoma indication } \\
\hline - Protection of anastomosis & 1.00 (ref) & \\
\hline - Anastomotic leakage & $.64(.19-2.17)$ & .477 \\
\hline - Acute colonic obstruction & $.53(.05-5.78)$ & .604 \\
\hline - Other Benign ${ }^{a}$ & $.99(.13-7.69)$ & .990 \\
\hline Type of stoma & & .584 \\
\hline Colostomy & $1.36(.46-4.05)$ & \\
\hline Ileostomy & 1.00 (Ref) & \\
\hline Surgical site infection & & .307 \\
\hline Yes & $2.06(.52-8.22)$ & \\
\hline No & 1.00 (Ref) & \\
\hline Prolapse & & $.042^{*}$ \\
\hline Yes & $3.27(1.04-10.27)$ & \\
\hline No & 1.00 (Ref) & \\
\hline Parastomal hernia & & $.020^{*}$ \\
\hline Yes & $5.08(1.30-19.85)$ & \\
\hline No & 1.00 (Ref) & \\
\hline Hypertension & & $.022 *$ \\
\hline Yes & $2.52(1.14-5.54)$ & \\
\hline No & 1.00 (Ref) & \\
\hline Emergency surgery & & .951 \\
\hline Yes & $1.06(.15-7.73)$ & \\
\hline No & 1.00 (Ref) & \\
\hline Malignancy & & .779 \\
\hline Yes & $1.18(.37-3.71)$ & \\
\hline No & 1.00 (Ref) & \\
\hline Type of stoma in pts with malignant disease & & $.034^{*}$ \\
\hline Colostomy & $5.05(2.28-11.23)$ & \\
\hline Ileostomy & 1.00 (Ref) & \\
\hline
\end{tabular}

* Independent risk factor for the development of an incisional hernia following stoma reversal, ${ }^{\mathrm{a}}$ Other Benign includes diverticulitis, slow transit colon and anal fistulas, Abbreviations: BMI = Body Mass Index, pts = patients

stoma reversal, hypertension, undergoing adjuvant therapy, emergency stoma construction, having a malignancy as underlying disease and indication for stoma construction were identified as possible risk factors and therefore included in the multivariate logistic regression model (Table 3).

The subsequent multivariate analysis identified increasing BMI, stoma prolapse, parastomal hernia, hypertension and colostomy reversal in patients with an underlying malignancy as independent risk factors for the development of an incisional hernia. (Table 3) The type of stoma (colo- versus ileostomy) was not a significant risk factor in patients without an underlying malignancy. Other interaction terms tested were; BMlxHypertension, MalignancyxWoundinfection, AgexHypertension, AgexMalignancy. These terms did not result in a significant outcome; interaction between these variables was therefore ruled out. 


\section{DISCUSSION}

Incisional hernia at the previous stoma site occurred in approximately one third of all patients after stoma reversal. This seems an appropriate reflection of the true patient population experiencing incisional herniation, as this was a consecutive cohort of patients who had a stoma for a variety of reasons. Many patients wished to undergo surgical correction of these hernias. Higher BMI, a stoma prolapse, a parastomal hernia, hypertension and colostomy reversal in patients with an underlying malignant disease were identified as independent risk factors for incisional hernia development.

The high incisional hernia rate observed in this study is concordant with the rate observed in recent studies that specifically focused on this problem. ${ }^{6,7,8}$ Older studies have reported markedly lower incisional hernia rates following stoma closure, ${ }^{3}$ but tended to be focused mainly on short-term outcomes (complications, anastomotic leakage) following stoma reversal. Incisional hernia rate was a secondary outcome and registration could therefore have been less precise as follow-up was short and incomplete. ${ }^{6}$

Furthermore, it is remarkable that hernia rates following stoma reversal were substantially higher than those seen with midline laparotomy wounds (around $10 \%$ ). ${ }^{11,12} \mathrm{~A}$ possible explanation could be that the fascial defect is larger in stoma wounds. In addition, the defect is oval shaped and closure of these specific wounds may result in increased tension on the abdominal wall compared to laparotomy incisions. Tension may impair scarring of the fascia. Furthermore, stoma reversal is considered a surgery in a possibly contaminated area. This might lead to higher surgical site infection rates, which is thought to be a risk factor for incisional hernia development. ${ }^{17,18}$ The current study did not identify surgical site infection as an independent risk factor; this might be explained by a low incidence of surgical site infection in our study do to the use of a purse string closure. This closure technique is known to markedly decrease surgical site infection following stoma reversal. ${ }^{19}$

It is suggested that a continuous suture technique with slowly absorbable monofilament suture material is preferred to prevent incisional hernias in laparotomy wounds. ${ }^{20}$ This preference however, is based on the number of wound infections as primary outcome. Studies on preferred suturing materials or whether a continuous or intermittent technique is preferred in order to prevent incisional hernia are not available. It is known however, that a small bites suture technique $(5 \mathrm{~mm})$ is more effective than a large bites $(1 \mathrm{~cm})$ technique in preventing incisional hernias. ${ }^{21}$ Several different suture techniques and materials have been used in the current study without clear superiority for a particular technique (intermittent/continuous) or suture material. Unfortunately, it was not possible to retrieve whether sutures were placed with small or large bites from the surgical reports. The most effective closure technique for stoma wounds therefore remains unclear.

The surgical correction rate of diagnosed hernias was high in the present series compared to most previous studies. ${ }^{6}$ This may be the result of longer follow-up. In addition, a surgeon examined all patients, which could have lowered the threshold for re-intervention. Another study with a similar follow-up also showed re-intervention rates of around $70 \%{ }^{14}$ 
Several previous studies have tried to identify possible risk factors for incisional hernia development following stoma reversal. High BMI and having a temporary colostomy have been identified as significant risk factors in several of these studies. ${ }^{6,8,22,23}$ Parastomal hernia, stoma prolapse and hypertension have not been identified previously. Most of these factors were not included in other studies. In addition, previous studies had a considerably smaller sample size. Parastomal hernia or stoma prolapse increases the size of the existing fascial defect. This could further weaken the abdominal wall, making it more prone to incisional hernia development. The effects of hypertension on wound healing are less clear, possibly chronic microvascular changes secondary to hypertension could impair adequate tissue perfusion, hereby reducing proper wound healing and contributing to possible wound dehiscence. ${ }^{22}$ It is likely that other factors identified in previous studies, such as diabetes mellitus and wound infection, are also independent risk factors for hernia development. ${ }^{23}$ The low incidence of these factors in this study might explain why they were not identified.

The most important limitation of this study is its retrospective design, which is prone to selection bias. Not all patients were followed for more than twelve months with imaging. In these patients, smaller asymptomatic incisional hernias could have been missed. This may have led to an underestimation of the true incisional hernia rate. ${ }^{2,24}$ Patients with risk factors have a higher risk of developing an incisional hernia. These patients would therefore benefit most from prophylactic measures. Stoma reversal in these high-risk patients could even be regarded as a hernia repair instead of a simple fascia closure. ${ }^{25}$ This might imply placement of a (prophylactic) mesh in order to prevent future herniation at the stoma incision site. Several retrospective studies of prophylactic mesh placement showed promising results. Randomized controlled trials, however, are lacking but should be undertaken to see if mesh placement reduces incisional hernia incidence at the stoma site without increasing surgical site infection. 


\section{REFERENCES}

1. Chow A, Tilney HS, Paraskeva P, Jeyarajah S, Zacharakis $\mathrm{E}$, Purkayastha $\mathrm{S}$. The morbidity surrounding reversal of defunctioning ileostomies: a systematic review of 48 studies including 6,107 cases. Int J Colorectal Dis; 2009; 24:711-23

2. Cingi A, Cakir T, Sever A, Aktan AO. Enterostomy site hernias: a clinical and computerized tomographic evaluation. Dis Colon Rectum; 2006; 49(10):1559-63.

3. Tilney HS, Sains PS, Lovegrove RE et. Comparison of outcomes following ileostomy versus colostomy for defunctioning colorectal anastomoses. World J Surg 2007;31:1142-1151

4. Bakx R, Busch OR, Bemelman WA et al. Morbidity of temporary loop ileostomies. Dig Surg 2004;21:277-281 10. Rondelli F, Reboldi P, Rulli A et al. Loop ileostomy versus loop colostomy for fecal diversion after colorectal or coloanal anastomosis: a meta-analysis. Int J Colorectal Dis 2009;24:479-488

5. Guenaga KF, Lustosa SA, Saad SS et al. lleostomy or colostomy for temporary decompression of colorectal anastomosis. Cochrane Database Syst Rev 2007;1:CD004647

6. Bhangu A, Nepogodiev D, Futaba K. Systematic review and meta-analysis of the incidence of incisional hernia at the site of stoma closure. World J Surg; 2012; 36:973-983

7. Bhangu A, Fletcher L, Kingdon S, Smith E, Nepogodiev $D$, Janjua U. A clinical and radiological assessment of incisional hernias following closure of temporary stomas. Surgeon; 2012; 10(6):321-5

8. Schreinemacher MH, Vijgen GH, Dagnelie PC, Bloemen $J G$, Huizinga BF, Bouvy ND. Incisional hernias in temporary stoma wounds: a cohort study. Arch Surg; 2011; 146(1):94-9

9. Hamel MB, Henderson WG, Khuri SF, Daley J. Surgical outcomes for patients aged 80 and older: morbidity and mortality from major noncardiac surgery. J Am Geriatr Soc; $2005 ;$ 53:424-429

10. McGugan E, Burton H, Nixon SJ, Thompson AM. Deaths following hernia surgery: room for improvement. $J R$ Coll Surg Edinb; 2000; 45:183-186

11. Kuhry E, Schwenk WF, Gaupset R, Romild U, Bonjer HJ. Long-term results of laparoscopic colorectal cancer resection. Cochrane Database Syst Rev; 2008; (2):CD003432

12. Kingsnorth $A$, LeBlanc K. Hernias: inguinal and incisional. Lancet; 2003; 362(9395): 1561-1571
13. Maggiori L, Moszkowicz D, Zappa M, Mongin C, Panis Y. Bioprosthetic mesh reinforcement during temporary stoma closure decreases the rate of incisional hernia: A blinded, case-matched study in 94 patients with rectal cancer. Surgery; 2015; 158(6): 1651-1657

14. van Barneveld KW, Vogels RR, Beets GL, Breukink SO, Greve JW, Bouvy ND, et al. Prophylactic intraperitoneal mesh placement to prevent incisional hernia after stoma reversal: a feasibility study. Surg Endosc; 2014; 28:1522-7

15. Wada $Y$, Miyoshi N, Ohue M, Noura S, Fujino S, Sugimura K, Akita H, Motoori M, Gotoh K, Takahashi H, Kobayashi S, Ohmori T, Fujiwara Y, Yano M. Comparison of surgical techniques for stoma closure: A retrospective study of purse-string skin closure versus conventional skin closure following ileostomy and colostomy reversal. $\mathrm{Mol}$ Clin Oncol. 2015;3(3):619-622

16. Integraal Kankercentrum Nederland. National working group on gastrointestinal cancers. Guideline colon cancer 2.0. Available at: http://www.oncoline.nl/ coloncarcinoom. Accessed October 22nd 2017

17. Murray BW, Cipher DJ, Pham T, Anthony T. The impact of surgical site infection on the development of incisional hernia and small bowel obstruction in colorectal surgery. Am J Surg. 2011;202(5):558-60.

18. Walming S, Angenete E, Block M, Bock D, Gessler B, Haglind E. Retrospective review of risk factors for surgical wound dehiscence and incisional hernia. BMC Surg. 2017;22;17(1):19

19. Camacho-Mauries D, Rodriguez-Díaz JL, Salgado-Nesme N, González QH, Vergara-Fernández O. Randomized clinical trial of intestinal ostomy takedown comparing pursestring wound closure vs conventional closure to eliminate the risk of wound infection. Dis Colon Rectum. 2013;56(2):205-11

20. Heger P, Pianka F, Diener MK; Mihaljevic AL. Current standards of abdominal wall closure techniques: Conventional suture techniques; Chirurg; 2016;87(9):737-43

21. Deerenberg EB, Harlaar J, Steyerberg EW, Lont HE, van Doorn HC, Heisterkamp J, Wijnhoven BP, Schouten WR, Cense HA, Stockmann HB, Berends FJ, Dijkhuizen FPH, Dwarkasing RS, Jairam AP, van Ramshorst $G H$, Kleinrensink GJ, Jeekel J, Lange JF. Small bites versus large bites for closure of abdominal midline incisions (STITCH): a double-blind, multicentre, randomised controlled trial. Lancet. 2015;26;386(10000):1254-1260

22. Van Ramshorst GH, Nieuwenhuizen J, Hop WC. Abdominal wound dehiscence in adults: development and validation of a risk model. World J Surg 2010: $34: 20-27$ 
23. Sharp SP, Francis JK, Valerian BT, Canete JJ, Chismark $A D$, Lee EC. Incidence of ostomy site incisional hernias after stoma closure. The American surgeon; 2015; 12(81):1244-1248

24. Bhangu A, Fletcher L, Kingdon S, Smith E, Nepogodiev $D$, Janjua U. A clinical and radiological assessment of incisional hernias following closure of temporary stomas. Surgeon; 2012; 10(6):321-5.

25. Cingi A, Solmaz A, Attaallah W, Aslan A, Aktan AO. Enterostomy closure site hernias: a clinical and ultrasonographic evaluation. Hernia; 2008; 12:401-405 


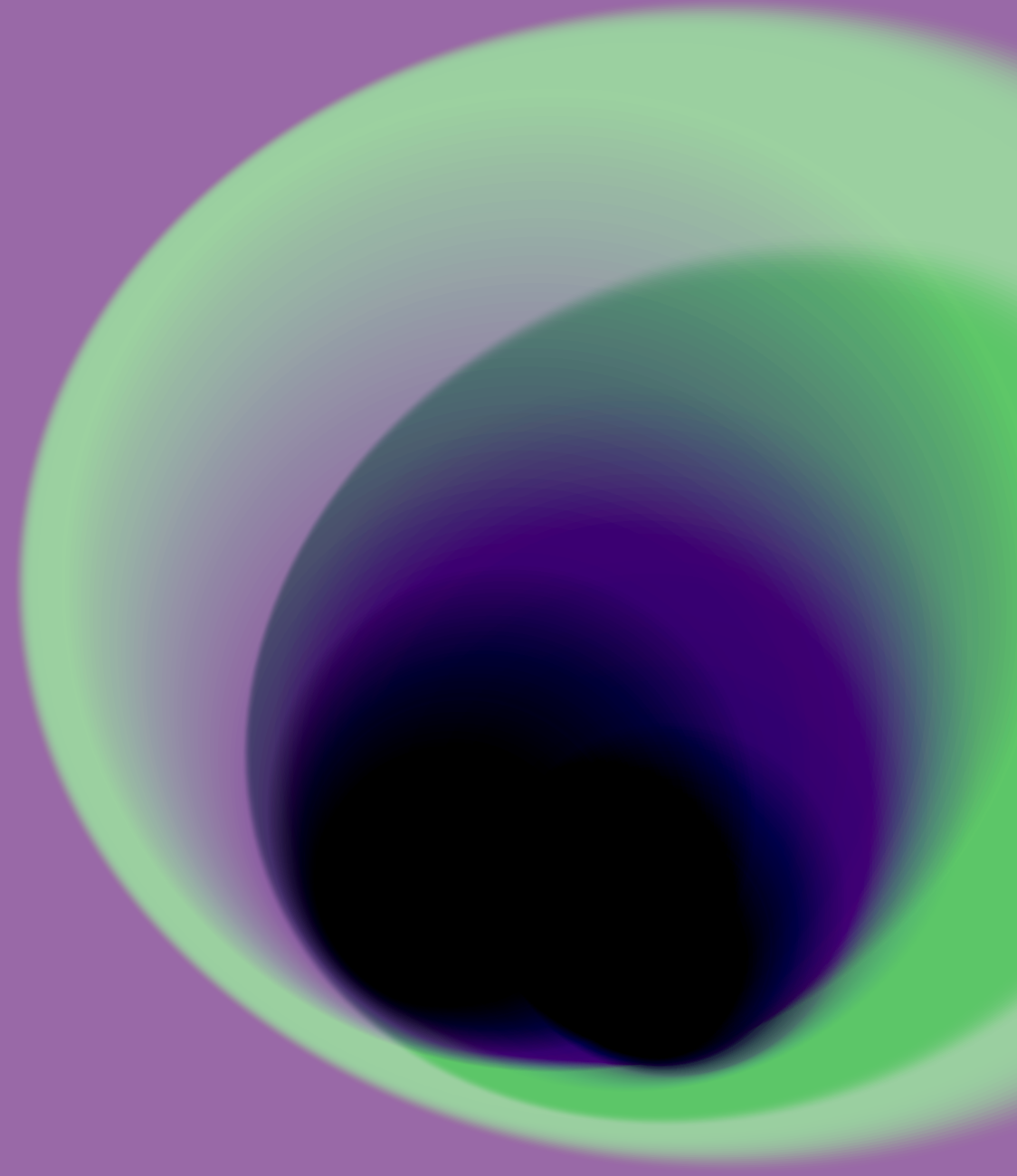





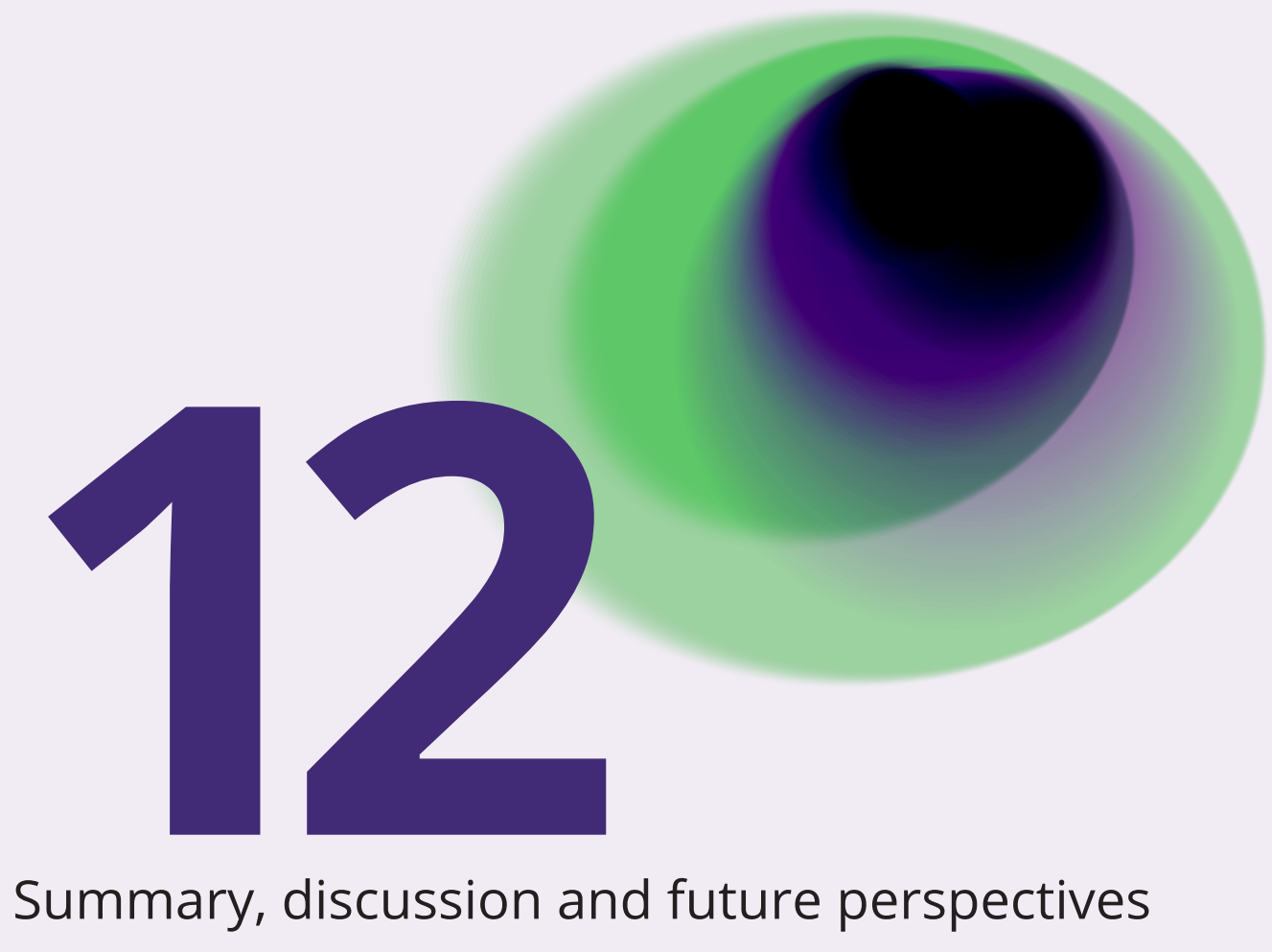


The management of obstructive colon cancer is a much debated issue. This thesis addresses several gaps in the current knowledge in order to optimize treatment strategies. The content is divided in three main parts, with specific research questions in each part. The summery, discussion, clinical implications and limitations will be addressed accordingly in this chapter.

\section{PART I - RIGHT-SIDED COLONIC OBSTRUCTIONS}

Emergency resection is the standard of care for patients with acute right-sided obstructive colon cancer. ${ }^{1}$ Even though bridge-to-surgery approaches have been extensively studied in left-sided obstructive colon cancer and the short-term benefits are well established, studies investigating this strategy for proximal tumors are scarce. ${ }^{2}$ Morbidity and mortality rates in patients with a right-sided obstruction of up to $48 \%$ and $18.5 \%$, respectively, have been reported. ${ }^{3}$ These high numbers are comparable to those found in patients with a left-sided obstruction, and have initiated the search for a safer alternative treatment approach, such as a bridge to surgery. Therefore, the first research question of this thesis was;

\section{Is a bridge to surgery approach also beneficial for patients with right-sided obstructive colon cancer?}

A first step in exploring this research question was evaluating the existing literature. We conducted a systematic literature search, which is presented in chapter $\mathbf{2}$. Interestingly, no comparative studies were found whatsoever. In addition, no studies investigating stoma construction as bridge to surgery in proximal malignant obstructions could be identified, highlighting the lack of data on this subject.

Out of the fourteen included cohort studies in chapter 2; ten evaluated patients that underwent emergency resection, whereas the other four reported on SEMS placement as bridge to surgery. In order to provide preliminary data on the safety of SEMS placement in patients with a proximal obstruction, a comparative analysis between the outcomes of these cohort studies was conducted. Significantly lower major morbidity ( 23.9 vs. $0.8 \%, p=0.009$ ) and mortality ( 10.8 vs. $0.0 \%, p=0.049$ ) rates were found in patients initially treated with SEMS. Also, a trend towards more primary anastomoses and fewer permanent stomas in the SEMS group were reported. Although these outcomes suggest that SEMS as bridge to surgery might decrease morbidity and mortality rates, the results should be interpreted with caution due to the low methodological quality of all included studies. The most important drawback was the fact that none of the included studies were comparative and all of them were retrospective with a resulting risk for selection bias. However, even though no definite conclusions can be drawn due to the previously mentioned limitations, the outcomes presented in chapter $\mathbf{2}$ do indicate a possible role for SEMS placement in right-sided obstructions and provide a starting point for future studies.

Since the publication of chapter $\mathbf{2}$, four comparative studies have been publilshed comparing SEMS placement as bridge to surgery with emergency resection for right-sided obstructions. 
In 2016, Kye et al. conducted the first comparative study. Twenty-five SEMS patients were compared to 49 emergency resection patients on short-term outcomes. ${ }^{4}$ Despite a significantly higher laparoscopic resection rate, no significant differences were found regarding all other short-term outcomes in this study. The outcomes of a similar study by Ji et al, comparing 14 SEMS with 25 emergency resection patients, confirmed the higher laparoscopy rate, and also showed a significantly shorter hospital stay in the SEMS group. ${ }^{5}$ Mortality and morbidity outcomes were not significantly different. Even though these studies are also retrospective and included a limited number of patients, they are the first comparative studies available and outcomes are similar. These outcomes therefore strengthen the previously formulated hypothesis that SEMS placement in the proximal colon seems safe.

In order to determine the use and corresponding outcomes of the different treatment strategies in the Netherlands for right-sided colonic obstructions, a population-based analysis was conducted in 2017 (chapter 3). In total, 1860 patients with an acute right-sided colon obstruction were identified in the Netherlands between 2009 and 2014. The vast majority of these patients was treated according to the golden standard; emergency resection (95.4\%). SEMS placement (2.4\%) or stoma construction (2.3\%) as a bridge to surgery was conducted in the remaining patients. Interestingly, this was, and to date still is, the only study that investigates stoma construction as bridge to surgery for right-sided colonic obstructions. A significant difference in 30-day mortality in favor of the bridge to surgery groups was found; with $8.8 \%$ mortality in the emergency resection group compared to $2.4 \%$ in both bridge to surgery groups $(p=0.04)$. In patients over 70 years, the difference in mortality rates was even more evident. Furthermore, the higher rate of laparoscopic resections following a bridge to surgery approach was once again confirmed. No other short-term benefits were reported in the stoma group. In the SEMS group, complication rates were found to be significantly lower than in the emergency resection group ( 27.3 vs. $39.6 \%, p=0.05$ ).

The outcomes of this large-population based study once again strengthen the hypothesis that SEMS placement in the proximal colon is safe, and also suggest that it could be a more beneficial approach. When interpreting these results, however, it is important to keep in mind that these conclusions are still based on retrospective studies only, which means that treatment allocation was not random. Selection bias might therefore have affected the results.

It is important to realize, that SEMS placement in the proximal colon is considered to be technically challenging. ${ }^{6-9}$ The difficulty is thought to be due to the long distance from the anus and tortuosity of the bowel, which prevents adequate placement of the stent. ${ }^{6,7}$ Also, malignant obstructions tend to be more severe since fecal contents are fluid at this point and an absolute obstruction is required to cause symptoms. ${ }^{8}$ Two studies have compared technical success rates of stent placement between distal and proximal obstructions. One study underlined the previously stated hypothesis, and found a significantly lower technical success rate in the proximal colon. ${ }^{9}$

However, an other, more recent study by Schoonbeek et al. (2018) found no difference in technical success rate. ${ }^{10} \mathrm{~A}$ sub-analysis in one of our own studies, comparing SEMS placement 
in the distal and proximal colon, also found no difference in technical and clinical success rate, as well as complication rate (chapter 4). An important difference between the first and last two studies was the level of experience of the endoscopist, which was considerably higher in the two most recent studies. This emphasizes the importance of experience in SEMS placement, which has been a known risk factor for failure of SEMS placement as well as perforation in left-sided obstructions. ${ }^{11-13}$ Interestingly, when technical success is achieved, SEMS-related complication and patency rates are similar regardless of tumor location..$^{9,10}$ In experienced hands, SEMS placement in the proximal colon therefore seems safe and might be technically less demanding than previously thought.

In order to provide more objective data on the safety of SEMS in the proximal colon, we conducted a case-matched analysis comparing SEMS to emergency resection (chapter 4). Consecutive SEMS patients were identified from one center, in which this was the standard of care for colonic obstruction. These patients $(n=22)$ were then matched one-to-four on age, gender, ASA-score, tumor location, surgical approach and pTNM stage with patients from another center that underwent emergency resection. Clinical success rate for stent placement was $90.2 \%$. SEMS patients were found to have significantly fewer temporary stomas constructed but no other significant differences were reported.

Overall, as confirmed in chapter 3, emergency resection is still the standard of care for patients with a malignant obstruction in the proximal colon. Even though the number of studies increases, studies investigating a bridge to surgery approach remain scarce. The few studies (among which are chapter 3 and 4 ) that have investigated bridge to surgery modalities indicate a possible role for this approach. SEMS placement appears to be feasible and safe and might even be associated with several advantages. In the current guidelines, a bridge to surgery approach is not recommended for right-sided obstructions. Given the outcomes of this thesis and other recent literature, we suggest that the recommendation in guidelines on SEMS placement in left-sided obstructions should be extended to right-sided obstructions. ${ }^{14}$ This guideline recommends SEMS placement in elderly (>70 years) or frail (ASA-III/IV) patients. As both age and increased ASA score have also been reported as significant predictors for mortality and morbidity in patients with right-sided obstructions, direct application of the ESGE guideline seems adequate. ${ }^{15}$ Furthermore, an important condition for SEMS placement is sufficient experience of the endosopist with at least 20 colorectal stent procedures.

\section{PART II - LEFT-SIDED COLONIC OBSTRUCTIONS}

For left-sided obstructive colon cancer, both emergency resection as well as a bridge to surgery approach with SEMS or deviating colostomy are accepted treatment approaches in clinical practice. SEMS as bridge to surgery has been extensively studied, and meta-analyses found higher proportions of laparoscopic surgery, lower postoperative morbidity rates, fewer temporary stoma constructions and higher primary anastomosis rates when compared to emergency surgery. ${ }^{16}$ Even though these are encouraging outcomes, premature closure of 
several randomized trials due to a high stent-related complication rates and uncertainties regarding oncologic impact, have caused an ongoing debate about the safety of SEMS. ${ }^{17-20}$ This has led to a dramatic decrease in the use of SEMS. ${ }^{21,22}$ During this same time period, colostomy construction as bridge to surgery gained popularity. In theory, colostomy construction could offer all the benefits of a bridge to surgery approach, without the risk of SEMS-related complications or direct tumor manipulation. Therefore, our second research question is;

\section{Is the construction of a deviating colostomy as bridge to surgery a safe and valid alternative for emergency resection in patients with left-sided obstructive colon cancer?}

A systematic review regarding this question is presented in chapter 5 , in which pooling of eight comparative studies, including a total of 2424 patients, did not demonstrate any significant difference in mortality or morbidity rates. An important notion when interpreting these results is that six of the eight included studies were published between 1977 and 1995 . Only two studies where of a more recent date. In view of the advances in surgical techniques in the past decades, these results might therefore be outdated. A clear trend in mortality reduction following emergency resection from $15 \%$ around 30 years ago to $7 \%$ in more recent studies also indicates the important effect of advances in healthcare on mortality. ${ }^{21,23}$ Indeed, mortality rate following stoma construction were $1.1 \%$ and $3.7 \%$ in the two studies from 2009 and 2013 , compared to an overall $15.9 \%$ in the more dated studies (1977-1995). In order to investigate the outcomes of deviating colostomy as bridge to surgery using modern and minimally invasive surgical techniques, a cohort study of 146 patients that had a colostomy constructed for obstructive left-sided colon cancer between 2003 and 2015 was conducted (chapter 6). Eightyfive patients underwent colostomy construction as bridge to surgery, of whom two patients died (2.4\%). This is similar to the mortality rate reported in the other two recent studies and seems to be lower than the $7 \%$ mortality rate reported in recent studies for emergency surgery. ${ }^{21,24}$

Furthermore, the existing literature indicates that more primary anastomoses are constructed and a laparoscopic resection is more often possible in patients initially treated with stoma construction (chapter 5 and 6). ${ }^{21}$ In addition, the permanent stoma rate was only $18 \%$ in chapter 6 , which is lower than the $35.2 \%$ reported in one of the most recent meta-analysis investigating emergency surgery. ${ }^{16}$ The low permanent stoma rate is confirmed in a study by Oistamo et al. who found a $4 \%$ permanent stoma rate in the deviating stoma group. ${ }^{22} \mathrm{An}$ important drawback for many physicians regarding stoma construction as bridge to surgery is the negative effect that a stoma has on quality of life. ${ }^{25,26}$ However, as significantly more patients seem to end up with a permanent stoma in the emergency resection group, stoma construction as bridge to surgery should not be abandoned for this reason.

Even though high quality evidence is lacking, the initial data suggests that deviating stoma construction is safe. In addition, it might even be an alternative to emergency resection, since it offers all the benefits of a bridge to surgery approach and shows promising low mortality and morbidity rates. 
Apart from comparing colostomy construction to emergency surgery, it is also important to evaluate this approach in the light of the other bridge to surgery option; i.e. SEMS placement. Currently, no clear consensus exists on which decompression method is superior. ${ }^{21,22}$ Therefore the next research question in this thesis is;

\section{Which bridge to surgery approach should be preferred; SEMS placement or colostomy construction?}

In the current guidelines, SEMS placement as bridge to surgery is recommended over colostomy construction. Colostomy construction is only recommended when the obstruction is longer than $40 \mathrm{~mm}$, when no experienced endoscopist is available or when the patient is likely to be treated with angiogenesis inhibitors in the future, since these are known risk factors for stent perforation. ${ }^{15,27,28}$ The main reason SEMS is considered first choice is because this saves patients a stoma construction, which is thought to negatively affect QoL and to increase the total number of surgical interventions. ${ }^{25,29}$ However, this recommendation is not based on any data, as studies directly comparing both bridge to surgery approaches were not available when the guidelines were drafted in 2014. Since then, two studies comparing emergency resection with both SEMS placement as well as colostomy construction as bridge to surgery have been published. In addition, one study directly comparing both treatment strategies has been conducted, which is presented in chapter 7 .

Tanis et al conducted a large population based study, comparing 1485 emergency resection patients to 196 SEMS and 135 stoma patients. Ostaimo et al. conducted a similar, but much smaller study. As the main aim of both studies was to compare emergency surgery to a bridge to surgery approach, p-values for SEMS versus stoma analyses are lacking. Mortality was only presented by Tanis et al., but was comparable in the bridge to surgery groups, with $3.7 \%$ in the stoma group and $5.6 \%$ in the SEMS group. In addition, morbidity rate, the number of laparoscopic resections, primary anastomoses, temporary and permanent stomas were also similar in both bridge to surgery groups..$^{21,22}$ However, these are descriptive data only.

The first and only study that directly compares both bridge to surgery approaches, shows slightly different results (chapter 7). Even though mortality and morbidity rates and hospital stay were similar, the two bridge to surgery approaches seemed to differ on other endpoints. In the stoma group the number of surgical interventions and long-term complications was significantly higher. This difference is explained by a high percentage of incisional hernia repairs and the fact that colostomy reversal was performed during a third surgical procedure. In the SEMS group, significantly fewer resections were conducted laparoscopically.

Overall, both SEMS placement and stoma construction are effective decompression methods. SEMS placement seems to result in fewer surgical interventions and long-term complications. However, the consequences of SEMS-related perforations could be severe and safety with regard to the oncologic outcomes can as yet not be guaranteed. ${ }^{30,31}$ Shared decision making is therefore of utmost importance in determining which bridge to surgery approach is 
appropriate for an individual patient. Especially patients treated with curative intent might be hesitant to take any risks regarding long-term survival and prefer stoma construction over SEMS placement. Given the fact that permanent stoma rate is similar in both groups, no significant decrease in the quality of life in the stoma group is expected. Nonetheless, patients should be informed about the fact that they might have to undergo additional interventions.

Future studies with a higher methodological quality are necessary to further investigate which bridge to surgery approach is best. Currently, we are developing a propensity-score matched analysis comparing SEMS with stoma construction as bridge to surgery. Patients will be selected from a large retrospective national collaborative research project that has been conducted in the Netherlands in the past two years. Given the lack of appropriate data from randomized clinical trials (RCTs), this quasi-randomized population study will increase the quality of available evidence and should therefore provide more definite answers.

As discussed previously, a permanent stoma affects quality of life..$^{25,26}$ This is important, since many patients with colon cancer will be confronted with (temporary) stoma construction during their treatment course. In addition, patients presenting with a colorectal emergency, such as an acute colonic obstruction, have an even higher likelihood of stoma construction. ${ }^{32,33}$ Different stoma types exist, and it is unclear which type should be preferred. In case of a temporary stoma, the surgeon needs to decide between loop ileostomy or colostomy construction. Construction of the most optimal stoma type is essential, since this could decrease the complication rate and minimizes the impact on quality of life. However, no clear consensus exists on which stoma type should be preferred. This led to the next research question of this thesis.

\section{Which type of ostomy is preferable; ileostomy or colostomy?}

Of course, the indication for stoma construction is of great importance in this decision. For instance, it is unfeasible to construct an ileostomy in case of a left-sided obstructive colon cancer, since the ileo-ceacal valve will prevent adequate colonic decompression. This can lead to life threatening complications, such as a colonic perforation and should always be prevented. However, colonic obstruction is only the indication in a minority of cases for stoma construction. Overall, the most important indication for stoma construction is to protect a newly constructed anastomosis. ${ }^{34}$ For this, and almost every other indication, both an ileo- as well as a colostomy are viable options. Even though no recommendations are available in national guidelines, recent literature indicates an ileostomy should be preferred (chapter 8). Patients with an ileostomy have fewer wound infections, stoma prolapses, stoma retractions, parastomal hernias and incisional hernias and report a better quality of life. Indeed, this higher risk of a parastomal hernia has recently been shown again in a study with over 5000 patients. ${ }^{35}$ An important consideration, however, is whether patients with an ileostomy have a significantly risk of developing a high-output stoma with dehydration and even renal failure as a consequence. Even though renal failure is a rare complication, it is severe and could be 
life threatening. We therefore think that an ileostomy should not be first choice in patients with a higher risk of developing a high-output stoma, dehydration or renal failure. For instance, patients that are not self-sufficient, such as patients with dementia, mental retardation or bedridden patients, have a higher risk of developing dehydration. In addition, in case of a pre-existent compromised renal function there is also a higher risk of (severe) complications when a high-output stoma is present. ${ }^{36}$ Lastly, there are suggestions that adjuvant chemotherapy, and specifically capecitabine, increases the risk of chemotherapy induced diarrhea and high-output stoma. ${ }^{37,38}$ The choice between ileo- or colostomy construction, should therefore always be taken in the light of the individual patient and their future treatment perspectives. A helpful flowchart for making this decision is presented in chapter 8.

\section{PART III - LONG-TERM OUTSOMES}

As mentioned previously, the evidence concerning the beneficial effects of a bridge to surgery approach on short-term outcomes has increased substantially over the past years. Both SEMS placement and stoma construction as bridge to surgery seem to result in more laparoscopic resections, more primary anastomosis, fewer (temporary) stomas and possibly fewer complications (chapter 5-7). ${ }^{16}$

However, long-term data on both approaches is scarce and especially for SEMS placement concerns have been raised regarding the oncologic safety. ${ }^{30}$ Several papers have reported a higher rate of unfavorable histo-pathological findings following SEMS placement, such as an increase in circulating tumor cells and a higher rate of perineural and lymph node invasion. ${ }^{30,39-42}$ Even though the clinical relevance of these surrogate outcome measures is unclear, concerns regarding oncological safety are a major drawback in the implementation of SEMS placement as treatment approach for curative patients. This led us to the following research question:

\section{Is SEMS placement oncologically safe in patients treated with curative intent?}

Conducted RCTs investigating SEMS have mainly focused on short-term outcomes and many were terminated early due to high complication rates. ${ }^{31}$ Therefore, the available long-term data mainly consists of retrospective cohort studies. Two meta-analyses from 2015 and 2017, including a total of 11 and 17 studies, respectively, found no difference in survival or disease free survival. ${ }^{43,44}$ Because of the ongoing debate and the increasing body of relevant literature, an updated meta-analysis is presented in chapter 9, in which a pooled analysis of the data of 21 studies showed no significant difference regarding locoregional recurrence rate, overall and disease free survival. However, 16 of these 21 studies were retrospective and the results might therefore have been affected by selection bias. The fact that sensitivity analysis on 3-year overall survival revealed contradictory outcomes, with a trend towards worse survival in the SEMS group when only RCTs were taken into account, strengthens this hypothesis. More high quality data is therefore required to draw more definite conclusions. In chapter 10, 222 SEMS patients were matched one-to-two to 444 emergency resection patients on propensity score, 
creating a quasi-randomized design. Pre-treatment differences were already low between the unmatched groups, likely related to the fact that the vast majority of stents were placed as part of routine practice in dedicated SEMS centres, rather than selective allocation. Propensity score matching even further minimised selection bias. Interestingly, this study once again found no difference in long-term oncological outcomes, with similar locoregional recurrences, overall and disease free survival. Kang et al., comparing 109 emergency resection patients with 226 SEMS patients using propensity score as an adjusting factor in their analysis, presented similar outcomes. ${ }^{45}$ Even though data from RCTs is still lacking, these two studies are of substantially higher methodological quality than previous studies, have included a high number of patients and have an adequate follow-up. Therefore, the plausibility that SEMS placement is oncologically safe increases.

Two recent meta-analyses have shown that an increase in circulating tumor cells (CTCS) is significantly associated with a poor prognosis. ${ }^{46,47}$ However, extrapolation of these data to the setting of SEMS as BTS might be inappropriate, since SEMS induced CTCS might not be of similar oncological importance and only a transient phenomenon. Postsurgical circulating tumor cell increase has often been observed, but studies have also shown cell counts to normalize or even decrease in the period thereafter. ${ }^{48}$ In addition, Wind et al. have shown CTCs to significantly increase more following open surgery compared to laparoscopic surgery. ${ }^{49}$ As a laparoscopic approach is significantly more often possible in the SEMS group, this could counterbalance the CTC increase initially caused by SEMS placement. Overall, the long-term effects of CTC release by an intervention have not been definitively determined, and conclusions on survival should therefore not be based solely on these surrogate outcome parameters.

Even though evidence regarding the oncological safety of uncomplicated SEMS placement increases, SEMS-related perforations have been more clearly associated with an increase in loco-regional recurrence and possible worse survival in the past (chapter 10). ${ }^{50,51}$ However, given the results of Kang et al., as well as those presented in chapter 10, this does not seem to impact oncological outcome in the overall SEMS group. Worse survival due to perforation might therefore be counterbalanced by a positive impact in other SEMS subgroups. For instance, several studies have shown that postoperative complications also negatively affect oncological outcome..$^{52}$ Given its oncological impact, it remains crucial to minimize perforation risk. Apart from placement by an experienced endoscopist, SEMS placement should be reserved for patients with malignant strictures who will not be treated with bevacizumab in the future. ${ }^{53,54}$ In addition, post-stent dilatation should be avoided. ${ }^{54}$

The different risks and potential advantages related to both strategies should be considered when deciding on optimal management for the individual patient. For example, a young fit patient with a low operative risk profile and absence of competing risks of mortality (i.e. cardiovascular) might not want to take the risk of stent perforation and possible worse oncological outcomes. On the other hand, the $8 \%$ perforation risk might outweigh the high operative risk in elderly patients or patients with significant comorbidity. Also, the difference 
in risk of permanent stoma construction between age groups should be taken into account, underlining the need for patient-tailored treatment and shared decision-making in patients with obstructive colon cancer.

Even though colostomy construction as bridge to surgery has never been associated with worsened survival, it has been suggested to increase the overall number of (surgical) interventions required. Apart from the fact that many patients require an additional surgery to reverse their stoma, incisional hernias might also contribute to an increase in the number of interventions. In order to further investigate this, we came up with the following research question:

\section{What is the risk of incisional hernia development following stoma reversal?}

Several older studies have estimated the incidence of incisional hernia rate at the stoma incision site at $7 \% .{ }^{55-57}$ However, more recent studies, that had incisional hernia development as their primary outcome measure, report significantly higher rates, up to $35 \% .{ }^{58-60}$ Recent evaluation of a cohort of 318 consecutive patients who underwent stoma reversal also revealed an incisional hernia rate of $34.6 \%$. In $77.6 \%$ of these patients, the hernia was symptomatic and eventually $65.5 \%$ required surgical correction (chapter 11 ).

Thus, the true incidence of incisional hernia rate following stoma reversal seems to be around $35 \%$. This is substantially higher than the risk following midline laparotomy wounds, with an overall rate of around 10 per cent. ${ }^{61,62}$ These high rates have led some to conclude that stoma reversal should be regarded as hernia repair, rather than a simple fascia closure..$^{63}$ This would mean that placement of a (prophylactic) mesh could be an option, to prevent future herniation. Indeed, several retrospective studies investigating prophylactic mesh placement have shown promising results. ${ }^{59,64} \mathrm{~A}$ major drawback with this approach, however, is that the stoma reversal site is considered a possibly contaminated area and synthetic mesh placement might cause infectious problems. Therefore, it is important to identify the patients with the highest risk of incisional hernia development, as they could benefit most from prophylactic mesh placement.

Known risk factors for the development of an incisional hernia following stoma closure are a BMI over 25, having a colostomy (vs. ileostomy), hypertension, diabetes and having a parastomal hernia or stoma prolapse while the stoma was in situ (chapter 11). ${ }^{59,61,65,66}$ Prophylactic mesh placement in these patients might significantly reduce incisional hernia rate and hereby, additional surgical interventions. ${ }^{67}$ Unfortunately, randomized controlled trials on this subject are missing. Following publication of chapter 11, we decided to further investigate this and set up the PRINCE study. This is a randomized controlled trial including all patients that undergo stoma reversal. Patients are randomized to either conventional stoma closure or prophylactic synthetic mesh placement. Hopefully, the results of this trial will clarify whether prophylactic mesh placement is safe and feasible and should become standard in stoma reversal. 


\section{CONCLUSION}

Even though colorectal screenings programs might cause a decrease in the incidence of obstructive colon cancer, still approximately $8 \%$ of patients present with an acute obstruction. ${ }^{27}$ This thesis has given more insight into the management of obstructive colon cancer, but questions remain. Overall, patient-tailored treatment and shared decision making are key. The low mortality and morbidity rates in young $(<70)$ and fit (ASA score $1-2$ ) patients justify conducting an emergency resection, as this approach spares patients from multiple interventions. An important consideration is, however, the fact that this does lead to higher permanent stoma rates, which could decrease quality of life. Both bridge to surgery options are associated with significantly lower permanent stoma rates, also in younger patients. Therefore, a bridge to surgery approach and its possible advantages should be discussed with each individual patient. SEMS placement might be a good option in patients that do not want a stoma whatsoever, especially since it does not seem to affect oncological outcomes, as was previously thought. However, we only advise this option when an experienced endoscopist is available, since SEMS-related colonic perforation is associated with a higher risk of local recurrence.

In elderly and frail patients, a bridge to surgery approach should always be chosen over emergency resection. Not only are fewer patients left with a permanent stoma, but also mortality and morbidity rates are significantly lower. Whether patients are bridged with a stent or a deviating stoma should be individualised. SEMS as bridge to surgery should however only be offered when the necessary experience is available and in the absence of risk factors for SEMS-related perforation. Otherwise, deviating stoma construction should be the primary treatment choice. 


\section{REFERENCES}

1. Phillips RKS, Hittinger R, Fry JS, Fielding LP. Malignant large bowel obstruction. Br J Surg. 1985;72:296-302.

2. Bonin $\mathrm{EA}$, Baron TH. Update on the Indications and Use of Colonic Stents. Curr Gastroenterol Rep 2010;12(5):374-382

3. Tan KK, Sim R. Surgery for obstructed colorectal malignancy in an Asian population: predictors of morbidity and comparison between left- and right-sided cancers. J Gastrointest Surg 2010;14:295

4. Kye BH, Lee YS, Cho HM, Kim JG, Oh ST, Lee IK, Kang WK, Ahn CH5, Lee SC, Park JK, Kim HJ8. Comparison of LongTerm Outcomes Between Emergency Surgery and Bridge to Surgery for Malignant Obstruction in RightSided Colon Cancer: A Multicenter Retrospective Study. Ann Surg Oncol. 2016;23(6):1867-74

5. Ji WB, Kwak JM, Kang DW, Kwak HD, Um JW, Lee SI, Min BW, Sung NS, Kim J, Kim SH.Clinical benefits and oncologic equivalence of self-expandable metallic stent insertion for right-sided malignant colonic obstruction. Surg Endosc. 2017;31(1):153-158

6. Keymling M. Colorectal stenting. Endoscopy. 2003;35:234-238

7. Bhardwaj R, Parker MC. Palliative therapy of colorectal carcinoma: stent or surgery? Colorectal Dis. 2003;5:518-521

8. Stimac D. Colonic stents for the palliation of malignant colonic obstruction. Dig Dis. 2008;26:336-341

9. Cho YK, Kim SW, Lee BI, et al. Clinical outcome of selfexpandable metal stent placement in the management of malignant proximal colon obstruction. Gut Liver. 2011;5:165-70

10. Schoonbeek PK1, Genzel P, van den Berg EH, van Dobbenburgh OA, Ter Borg. .Outcomes of SelfExpanding Metal Stents in Malignant Colonic Obstruction are Independent of Location or Length of the Stenosis: Results of a Retrospective, Single-Center Series. Dig Surg. 2018;35(3):230-235

11. Small AJ, Coelho-Prabhu N, Baron TH. Endoscopic placement of self-expandable metal stents for malignant colonic obstruction: long-term outcomes and complication factors. Gastrointest Endosc 2010;71(3):560-572

12. Lee HJ, Park SJ, Cheon JH, Kim TI, Kim WH, Hong SP. What is the necessity of endoscopist for successful endoscopic stenting in patients with malignant colorectal obstruction? Int I Colorectal Dis. 2015;30(1):119-25
13. Williams,D.; Law,R.; Pullyblank,A.M. Colorectal stenting in malignant large bowel obstruction: the learning curve. Int J Surg Oncol., 2011:9:17848

14. van Hooft JE, van Halsema $E E$, Vanbiervliet $G$ et al. Selfexpandable metal stents for obstructing colonic and extracolonic cancer: European Society of Gastrointestinal Endoscopy (ESGE) Clinical Guideline. Endoscopy. 2014;46(11):990-1053

15. Mege D, Manceau G, Beyer-Berjot L, Bridoux V, Lakkis Z, Venara A, Voron T, Brunetti F, Sielezneff I, Karoui M; AFC (French Surgical Association) Working Group. Surgical management of obstructive right-sided colon cancer at a national level results of a multicenter study of the French Surgical Association in 776 patients. Eur J Surg Oncol. 2018 Jul 6

16. Arezzo A, Passera R, Lo Secco G, Verra M, Bonino MA, Targarona E, Morino M. Stent as bridge to surgery for left-sided malignant colonic obstruction reduces adverse events and stoma rate compared with emergency surgery: results of a systematic review and meta-analysis of randomized controlled trials. Gastrointest Endosc. 2017; 86:416-426

17. J.E. van Hooft, W.A. Bemelman, B. Oldenburg, A.W. Marinelli, M.F. Holzik, M.J. Grubben, M.A. Sprangers, M.G. Dijkgraaf, P. Fockens, Colonic stenting versus emergency surgery for acute left-sided malignant colonic obstruction: a multicentre randomised trial, Lancet Oncol. 2011;344e352

18. H.Y. Cheung, C.C. Chung, W.W. Tsang, J.C. Wong, K.K. Yau, M.K. Li, Endolaparoscopic approach vs conventional open surgery in the treatment of obstructing left-sided colon cancer: a randomized controlled trial, Arch. Surg. 2009;144;12;1127e1132,

19. L.A. Pirlet, K. Slim, F. Kwiatkowski, F. Michot, B.L. Millat, Emergency preoperative stenting versus surgery for acute left-sided malignant colonic obstruction: a multicenter randomized controlled trial, Surg. Endosc. 2011;25;1814e1821

20. C. Sabbagh, F. Browet, M. Diouf, et al., Is stenting as "a Bridge to Surgery" an oncologically safe strategy for the management of acute, left-sided, malignant, colonic obstruction?: a comparative study with a propensity score analysis, Ann. Surg. 2013;258;107e115

21. Tanis PJ, Paulino Pereira NR, van Hooft JE, Consten EC, Bemelman WA. Resection of obstructive left-sided colon cancer at a national level: a prospective analysis of short-term outcomes in 1,816 patients. Dig Surg 2015;32(5):317-324 
22. Öistämö E, Hjern F, Blomqvist L, Falkén $Y$, Pekkari $K$, Abraham-Nordling M. Emergency management with resection versus proximal stoma or stent treatment and planned resection in malignant left-sided colon obstruction. World Journal of Surgical Oncology. 2016;14(1):232

23. Tekkis PP, Kinsman R, Thompson MR, et al. The Association of Coloproctology of Great Britain and Ireland study of large bowel obstruction caused by colorectal cancer. Ann Surg 2004;240:76-81

24. J.K. Jiang, Y.T. Lan, T.C. Lin, W.S. Chen, S.H. Yang, H.S. Wang, S.C. Chang, J.K. Lin, Primary vs. delayed resection for obstructive left-sided colorectal cancer: impact of surgery on patient outcome, Dis. Colon Rectum 2008;51(3)306e311

25. Young CJ, De-Loyde KJ, Young JM, Solomon MJ, Chew $\mathrm{EH}$, Byrne CM, Salkeld G, Faragher IG. Improving quality of life for people with incurable large-bowel obstruction: randomized control trial of colonic stent insertion. Dis Colon Rectum 2015;58(9):838-849

26. Sprangers MA, Taal BG, Aaronson NK, te Velde A. Quality of life in colorectal cancer. Stoma vs. nonstoma patients. Dis Colon Rectum 1995;38(4):361-369

27. van Halsema $E E$, van Hooft JE, Small AJ, Baron $T H$, García-Cano J, Cheon JH, Lee MS, Kwon SH, MucciHennekinne S, Fockens P, Dijkgraaf MG, Repici A. Perforation in colorectal stenting: a meta-analysis and a search for risk factors. Gastrointest Endosc. 2014;79(6):970-82.e7

28. Boyle DJ, Thorn C, Saini A, Elton C, Atkin GK, Mitchell IC, Lotzof K, Marcus A, Mathur P. Predictive factors for successful colonic stenting in acute large-bowel obstruction: a 15-year cohort analysis. Dis Colon Rectum. 2015;58(3):358-62

29. Gandrup P, Lund L, Balslev I. Surgical treatment of acute malignant large bowel obstruction. Eur J Surg 1992; 158: $427-430$

30. Sabbagh C, Browet F, Diouf $M$ et al. Is stenting as "a bridge to surgery" an oncologically safe strategy for the management of acute, left-sided, malignant, colonic obstruction? A comparative study with a propensity score analysis. Ann Surg 2013;258:107-115

31. van Hooft JE, Bemelman WA, Oldenburg B et al. Colonic stenting versus emergency surgery for acute left-sided malignant colonic obstruction: a multicentre randomised trial. Lancet Oncol. 2011;12(4):344-52

32. Bayar B, Yılmaz KB, Akıncı M, Şahin A, Kulaçoğlu H. An evaluation of treatment results of emergency versus elective surgery in colorectal cancer patients. Turkish Journal of Surgery/Ulusal cerrahi dergisi. 2016;32(1):1117
33. Sjo OH, Larsen S, Lunde OC, Nesbakken A. Short term outcome after emergency and elective surgery for colon cancer. Colorectal Dis. 2009;11(7):733-9.

34. Scott-Conner, CEH. Chassin's operative strategy in colon and rectal surgery. New York: Springer; 2006:18

35. Andersen RM, Klausen TW, Danielsen AK, Vinther A, Gögenur I, Thomsen T. Incidence and risk factors for parastomal bulging in patients with ileostomy or colostomy: a register-based study using data from the Danish Stoma Database Capital Region. Colorectal Dis. 2018;20(4):331-340

36. Messaris E, Sehgal R, Deiling S, et al. Dehydration is the most common indication for readmission after diverting ileostomy creation. Dis Colon Rectum. 2012;55:175-80

37. Robertson JP, Wells $\mathrm{Cl}$, Vather R, Bissett IP. Effect of Diversion lleostomy on the Occurrence and Consequences of Chemotherapy-Induced Diarrhea. Dis Colon Rectum. 2016;59:194-200

38. Oliphant R, Czerniewski A, Robertson I, McNulty C, Waterston A, Macdonald A. The effect of adjuvant chemotherapy on stoma-related complications after surgery for colorectal cancer: a retrospective analysis. J Wound Ostomy Continence Nurs. 2015;42:494-8

39. Maruthachalam K, Lash GE, Shenton BK, Horgan AF. Tumour cell dissemination following endoscopic stent insertion. Br J Surg 2007;94:1151-1154, doi: NA

40. Kim HJ, Choi GS, Park JS et al. Higher rate of perineural invasion in stent-laparoscopic approach in comparison to emergent open resection for obstructing left-sided colon cancer. Int J Colorect Dis 2013;28:207-14

41. Poon J. Pang R. Law W. The impact of colonic stenting on tumor cell dissemination in colorectal cancer patients. Dis.Colon Rectum 2011;54:160,

42. Yamashita S, Tanemura M, Sawada G et al K. Impact of endoscopic stent insertion on detection of viable circulating tumor cells from obstructive colorectal cancer. Oncol Lett. 2018;15(1):400-406.

43. Matsuda A, Miyashita M, Matsumoto $S$ et al. Comparison of long-term outcomes of colonic stent as "bridge to surgery" and emergency surgery for malignant large-bowel obstruction: a meta-analysis. Ann Surg Oncol 2015;22(2):497-504

44. Ceresoli $\mathrm{M}$, Allievi N, Coccolini F et al. Long-term oncologic outcomes of stent as a bridge to surgery versus emergency surgery in malignant left side colonic obstructions: a meta-analysis. J Gastrointest Oncol 2017;8(5):867-876 
45. Kang SI, Oh HK, Yoo JS et al. Oncologic outcomes of preoperative stent insertion first versus immediate surgery for obstructing left-sided colorectal cancer. Surg Oncol. 2018;27(2):216-224

46. Tan $Y, W u$ H.The significant prognostic value of circulating tumor cells in colorectal cancer: A systematic review and meta-analysis. Curr Probl Cancer. 2017;29:S0147-0272(17)30129-0

47. Yang C, Zou K, Zheng L, Xiong B. Prognostic and clinicopathological significance of circulating tumor cells detected by RT-PCR in non-metastatic colorectal cancer: a meta-analysis and systematic review. BMC Cancer. 2017;717-725

48. Kaifi JT, Li G, Clawson G, Kimchi ET, Staveley-O'Carroll KF. Perioperative circulating tumor cell detection: Current perspectives. Cancer Biology \& Therapy. 2016;17(8):859-869

49. Wind J, Tuynman JB, Tibbe AG, Swennenhuis JF, Richel DJ, van Berge Henegouwen MI, Bemelman WA. Circulating tumour cells during laparoscopic and open surgery for primary colonic cancer in portal and peripheral blood. Eur J Surg Oncol. 2009;35(9):942-50

50. Sloothaak DA, van den Berg MW, Dijkgraaf MG, Fockens P, Tanis PJ, van Hooft JE, Bemelman WA; collaborative Dutch Stent-In study group. Oncological outcome of malignant colonic obstruction in the Dutch Stent-In 2 trial. Br J Surg. 2014;101(13):1751-7

51. Avlund TH, Erichsen R, Ravn S, Ciplys Z, Andersen JC, Laurberg S, Iversen LH.The prognostic impact of bowel perforation following self-expanding metal stent as a bridge to surgery in colorectal cancer obstruction. Surg Endosc. 2018;32(1):328-336

52. Ha GW, Kim JH, Lee MR. Oncologic Impact of Anastomotic Leakage Following Colorectal Cancer Surgery: A Systematic Review and Meta-Analysis. Ann Surg Oncol. 2017;24(11):3289-3299

53. Small AJ, Coelho-Prabhu N, Baron TH. Endoscopic placement of self-expandable metal stents for malignant colonic obstruction: long-term outcomes and complication factors. Gastrointest Endosc 2010;71(3):560-572

54. van Halsema EE, van Hooft JE, Small AJ et al. Perforation in colorectal stenting: a meta-analysis and a search for risk factors. Gastrointest Endosc 2014;79(6):970-82

55. Tilney HS, Sains PS, Lovegrove RE, Reese GE, Heriot AG, Tekkis PP. Comparison of outcomes following ileostomy versus colostomy for defunctioning colorectal anastomoses. World J Surg 2007; 31: 1142-1151
56. Bakx R, Busch OR, BemelmanWA, Veldink GJ, Slors JF, van Lanschot JJ. Morbidity of temporary loop ileostomies. Dig Surg 2004; 21: 277-281

57. Guenaga KF, Lustosa SA, Saad SS, Saconato H, Matos D. lleostomy or colostomy for temporary decompression of colorectal anastomosis. Cochrane Database Syst Rev 2007; (1)CD004647

58. Bhangu A, Nepogodiev D, Futaba K. Systematic review and meta-analysis of the incidence of incisional hernia at the site of stoma closure. World J Surg 2012; 36: 973-983

59. Bhangu A, Fletcher L, Kingdon S, Smith E, Nepogodiev $D$, Janjua U. A clinical and radiological assessment of incisional hernias following closure of temporary stomas. Surgeon 2012; 10: 321-325

60. Schreinemacher MH, Vijgen GH, Dagnelie PC, Bloemen $J G$, Huizinga BF, Bouvy ND. Incisional hernias in temporary stoma wounds

61. Kuhry E, Schwenk WF, Gaupset R, Romild U, Bonjer HJ. Long-term results of laparoscopic colorectal cancer resection. Cochrane Database Syst Rev 2008; (2) CD003432

62. Kingsnorth A, LeBlanc K. Hernias: inguinal and incisional. Lancet 2003; 362: 1561-1571

63. Cingi A, Solmaz A, AttaallahW, Aslan A, Aktan AO. Enterostomy closure site hernias: a clinical and ultrasonographic evaluation. Hernia 2008; 12: 401-405

64. Maggiori L, Moszkowicz D, Zappa M, Mongin C, Panis Y. Bioprosthetic mesh reinforcement during temporary stoma closure decreases the rate of incisional hernia: a blinded, case-matched study in 94 patients with rectal cancer. Surgery 2015; 158: 1651-1657

65. Van Ramshorst GH, Nieuwenhuizen J, HopWC Abdominal wound dehiscence in adults: development and validation of a risk model. World J Surg 2010; 34: $20-27$

66. Sharp SP, Francis JK, Valerian BT, Canete JJ, Chismark $A D$, Lee EC. Incidence of ostomy site incisional hernias after stoma closure. Am Surg 2015; 81: 1244-1248

67. Warren JA, Beffa LR, Carbonell AM, Cull J, Sinopoli B, Ewing JA, McFadden C, Crockett J, Cobb WS Prophylactic placement of permanent synthetic mesh at the time of ostomy closure prevents formation of incisional hernias. Surgery. 2018;163(4):839-8 

Summary, discussion and future perspectives in Ducth

(Nederlandse samenvatting, discussie en toekomstperspectieven)

Acknowledgements | Dankwoord

Publication List

Curriculum Vitae 


\section{SUMMARY, DISCUSSION AND FUTURE PERSPECTIVES IN DUCTH}

(Nederlandse samenvatting, discussie en toekomstperspectieven)

Darmkanker is wereldwijd een van de meest voorkomende kankersoorten en nog steeds neemt de incidentie jaarlijks toe. ${ }^{1}$ In Nederland worden ongeveer 15.000 nieuwe gevallen gediagnostiseerd per jaar. ${ }^{2}$ De meeste patiënten presenteren zich met niet-specifieke symptomen zoals gewichtsverlies, vermoeidheid of een veranderd ontlastingspatroon. Middels aanvullende tests kan dan de diagnose darmkanker worden gesteld. ${ }^{3} 9$ tot $13 \%$ van de patiënten met darmkanker presenteert zich initieel met klachten van een acute afsluiting van de dikke darm; ook wel een darmobstructie. ${ }^{4}$ Deze patiënten behoeven binnen 24 uur behandeling, om te voorkomen dat er dusdanig veel druk op de darm komt te staan dat deze perforeert.

De meeste patiënten die zich presenteren met een acute darmobstructie zijn van oudere leeftijd en in een algeheel slechte conditie doordat ze enkele dagen niet adequaat konden eten en drinken..$^{5,6}$ Daarnaast weten we dat deze patiënten vaker metastasen (uitzaaiingen) of verder gevorderde ziekte hebben. ${ }^{7-9}$ Deze factoren dragen allemaal bij aan een verhoogd operatierisico.

Op dit moment is de gouden standaard behandeling voor een obstructie door darmkanker acute resectie van de tumor. Deze behandeling is echter geassocieerd met een hoog complicatiegehalte van $60 \%$ en een mortaliteit van $22 \% .{ }^{10}$ In ouderen met co-morbiditeit is de mortaliteit zelfs nog hoger; tot wel $32,2 \% .^{11}$

Het hoge aantal complicaties en postoperatieve sterfgevallen heeft geleid tot de zoektocht naar alternatieve behandelmogelijkheden. Een brug tot electieve resectie aanpak zou dit alternatief kunnen zijn. Hierbij wordt middels een minimaal invasieve ingreep de passage van ontlasting weer hersteld, waarbij de tumor op een later en gepland moment verwijderd kan worden. In de tussenliggende tijd kan de conditie van de patiënt geoptimaliseerd worden voor de aankomende grote abdominale operatie. Een brug tot electieve resectie kan gecreëerd worden door het plaatsen van een stent langs de obstruerende tumor of door het aanleggen van een dubbelloops stoma voor de tumor. Beide methoden zorgen ervoor dat de ontlasting kan worden afgevoerd, waardoor het risico op darmperforatie wordt weggenomen.

Dit proefschrift behandelt verschillende onbeantwoorde vragen omtrent de beste behandeling van obstructieve darmkanker. Het proefschrift is ingedeeld in drie delen, waarbij in elk deel specifieke onderzoeksvragen worden behandeld. Hieronder vindt $u$, per onderzoeksvraag, een samenvatting van de onderzoeken op dit gebied waarbij we de resultaten hiervan bespreken in het kader van de meest recente literatuur.

\section{DEEL I - RECHTSZIJDIGE DARMOBSTRUCTIES}

De behandeling voor obstruerende darmkanker is afhankelijk van de locatie van de tumor. Hierbij worden tumoren ingedeeld in rechtszijdig (proximaal) en linkszijdig (distaal). Proximale tumoren bevinden zich in het caecum, het colon ascendens, de flexura hepatica of het colon transversum. Tumoren worden als distaal geclassificeerd wanneer deze zich in de flexura 
lienalis, colon descendens of het sigmoïd bevinden. Een acute resectie is op dit moment de standaard behandeling voor patiënten met een proximale obstructie door darmkanker. ${ }^{12}$ Ondanks dat de brug tot electieve resectie aanpak uitgebreid is onderzocht voor linkszijdige obstructieve tumoren zijn er maar weinig studies die onderzoeken of deze aanpak ook gunstig kan zijn bij rechtszijdige obstruerende tumoren. ${ }^{13}$ Dit is opmerkelijk omdat ook bij patiënten met rechtszijdige obstructies het aantal complicaties en de mortaliteit postoperatief erg hoog zijn. ${ }^{14}$ Bij linkszijdige tumoren hebben deze hoge morbiditeit en mortaliteit geleid tot de zoektocht naar een alternatieve behandelstrategie. Gezien de gelijkende uitkomsten na acute resectie, lijkt dat ook gerechtvaardigd voor patiënten met rechtszijdige obstructies. Zojuist genoemde heeft tot de eerste onderzoeksvraag van dit proefschrift geleid;

\section{Is een brug tot electieve resectie aanpak ook gunstig voor patiënten met een rechtszijdige obstructie door darmkanker?}

Ter beantwoording van deze vraag is allereerst een uitgebreid en systematisch literatuuronderzoek uitgevoerd, welke kan worden teruggevonden in hoofdstuk 2. Een interessante eerste bevinding was dat er geen vergelijkende studies ten aanzien van de behandeling van rechtszijdige darmobstructies bestonden. Daarnaast was er geen literatuur te vinden over stoma-aanleg als brug tot electieve resectie. Dit geeft nogmaals weer hoe beperkt de data op dit gebied is.

Van de veertien geïncludeerde studies in hoofdstuk 2, bespreken tien studies patiënten die een acute resectie hebben ondergaan; de andere vier studies bespreken patiënten die zijn behandeld met stentplaatsing als brug tot electieve resectie. Om toch een voorzichtige uitspraak te kunnen doen over de veiligheid van stentplaatsing bij rechtszijdige obstruerende tumoren, hebben we de uitkomsten van de cohort studies met elkaar vergeleken. Hierbij werden significant minder complicaties $(0,8$ vs $23,9 \%, p=0.009)$ en postoperatieve sterfgevallen $(0,0$ vs $10,8 \%, p=0.049)$ gezien in de stentgroep. Daarnaast werd ook een trend waargenomen richting meer primaire anastomosen en minder permanente stoma's in de stentgroep. Hoewel deze uitkomsten suggereren dat stent als brug tot electieve resectie tot gunstigere uitkomsten leidt, moeten deze resultaten voorzichtig geïnterpreteerd worden vanwege de lage methodologische kwaliteit van de geïncludeerde studies. Met name het feit dat geen van de studies vergelijkend is, vormt een belangrijke tekortkoming. Dit maakt het zeer waarschijnlijk dat selectiebias invloed heeft gehad op de resultaten. Hoewel er geen definitieve conclusies getrokken kunnen worden, lijkt er wel ruimte te zijn voor stentplaatsing in de behandeling van rechtszijdige obstruerende tumoren.

Sinds de publicatie van hoofdstuk 2 zijn er vier vergelijkende studies verschenen die stentplaatsing als brug tot electieve resectie vergelijken met acute resectie voor rechtszijdige tumoren. In 2016 is de eerste studie verschenen, uitgevoerd door Kye et al. Hierbij werden 25 stent patiënten vergeleken met 49 acute resectie patiënten op korte termijn uitkomsten. ${ }^{15}$ Ondanks dat meer patiënten laparoscopisch geopereerd konden worden in de stent groep, waren er geen andere significante verschillen aan te wijzen tussen de twee behandelgroepen. 
De uitkomsten van een vergelijkbare studie, uitgevoerd door Ji et al., waarbij 14 stent patiënten met 25 acute resectie patiënten werden vergeleken, toonden ook meer laparoscopische resecties in de stent groep. Hierbij werd de stent groep ook gekenmerkt door een significant kortere opnameduur. ${ }^{16}$ Ook in deze studies waren het aantal complicaties en de mortaliteit gelijk. Hoewel deze studies een beperkt aantal patiënten includeren, zijn het de enige twee beschikbare studies en laten ze gelijken uitkomsten zien. Hiermee sterken zij de hypothese dat stentplaatsing in rechtszijdige maligne obstructies veilig is.

Om een beter beeld te krijgen van het huidige behandelbeleid voor rechtszijdige obstructieve tumoren in Nederland, hebben wij in 2017 een populatiestudie verricht (hoofdstuk 3). In totaal werden 1860 patiënten geïdentificeerd, die zich tussen 2009 en 2014 in Nederland met een acute rechtszijdige darmobstructie presenteerden. Het merendeel van deze patiënten werd behandeld met de gouden standaard, acute resectie $(95,4 \%)$. De overige patiënten ondergingen stentplaatsing $(2,4 \%)$ of aanleg van een dubbelloops stoma $(2,3 \%)$ als brug tot electieve resectie. Tot op heden is dit nog steeds de enige studie die het aanleggen van een dubbelloops stoma als brug tot electieve resectie onderzoekt voor rechtszijdige obstruerende tumoren. De mortaliteit was significant lager in de brug tot electieve resectiegroepen $(2,4 \%$ voor zowel de stent- als de stomagroep versus $8.8 \%$ in de acute resectiegroep $(p=0.04))$. In oudere patiënten (>70 jaar) was dit verschil nog duidelijker. Daarnaast werd nogmaals bevestigd dat er meer laparoscopische resecties plaatsvonden in de stentgroep. Er waren geen andere verschillen in korte termijn uitkomsten tussen de stoma- en acute resectie-groep. In de stentgroep was het aantal complicaties echter wel significant lager dan in de acute resectiegroep (27,3 vs. 39,6\%, $\mathrm{p}=0.05)$.

De uitkomsten van deze grote populatiestudie maken de hypothese, stentplaatsing in het proximale colon is veilig, steeds aannemelijker. Sterker nog, stentplaatsing lijkt gunstigere korte termijn uitkomsten te geven ten opzichte van acute resectie. Het is echter belangrijk om hierbij in het achterhoofd te houden dat deze conclusies nog altijd alleen maar gevormd zijn op basis van retrospectieve studies, waarbij behandeltoewijzing niet willekeurig was. Selectiebias kan hier nog steeds invloed op hebben gehad.

Het is belangrijk om in gedachten te houden, dat stentplaatsing in de rechter helft van de darm technisch gecompliceerder is dan in het linker deel. ${ }^{17-19}$ Deze complexiteit komt voort uit de langere afstand vanaf de anus en de natuurlijke vorm van de darm. ${ }^{20,21}$ Daarnaast zijn obstructies in het proximale colon ernstiger, doordat de ontlasting op dat punt nog vloeibaar is. Er is een volledige afsluiting van de darm nodig om symptomen te veroorzaken. ${ }^{22}$ Twee studies hebben het technische succespercentage van stentplaatsing vergeleken in distale en proximale tumoren. Hoewel één studie de bovengenoemde hypothese sterkt, wordt in de meest recente studie geen verschil in technisch succes gevonden. ${ }^{23,24}$ Een sub analyse in één van onze eigen studies, waarbij stentplaatsing in het distale en proximale colon wordt vergeleken, laat ook geen verschil in uitkomsten zien. Een belangrijk verschil tussen de eerste en laatste twee studies, is het ervaringsniveau van de endoscopist. In de laatste twee studies was de endoscopist zeer ervaren in het plaatsen van colonstents, dit lijkt dus een belangrijke 
voorspeller voor technisch succes. Daarnaast is dit ook een bekend risico voor stentgerelateerde perforatie. ${ }^{25-27}$ Verder blijkt er, indien de stent eenmaal adequaat geplaatst is, geen verschil te zijn in het aantal stent-gerelateerde complicaties tussen proximale en distale stents. ${ }^{23,24}$ In ervaren handen, lijkt stentplaatsing in het proximale colon dan ook veilig en mogelijk zelfs minder moeilijk dan eerder werd gedacht.

Zoals in hoofdstuk $\mathbf{3}$ terug te zien is, is acute resectie vooralsnog de gouden standaard voor patiënten met een maligne obstructie van het rechtszijdige colon. Hoewel de literatuur langzaam toeneemt op dit gebied, is de beschikbare data nog steeds beperkt. De beschikbare data (waaronder hoofdstuk 3 en 4) lijkt er echter wel op te wijzen dat er een rol is voor de brug tot electieve resectie behandelstrategie. Stentplaatsing lijkt effectief en veilig en mogelijk zelfs voordelig als het gaat om korte termijn uitkomsten. In de huidige richtlijnen wordt een brug tot electieve resectie aanpak voor rechtszijdige obstructies niet genoemd. ${ }^{25}$ Kijkende naar de uitkomsten van dit proefschrift en de meest recente literatuur, zouden wij dan ook aanraden om de richtlijnen voor linkszijdige obstructies te verbreden naar rechtszijdige obstructies. Deze richtlijn raadt aan om een brug tot electieve resectieaanpak toe te passen bij oudere patiënten (>70 jaar) of patiënten met aanzienlijke co-morbiditeit. Aangezien zowel leeftijd als een hogere ASA-score ook aangetoonde voorspellers voor een hogere morbiditeit en mortaliteit zijn bij rechtszijdige tumoren, lijkt een directe vertaling van deze richtlijn adequaat. ${ }^{26}$ Een belangrijke voorwaarde hierbij is dat de endoscopist voldoende ervaring heeft met colon stentplaatsing, waarbij een minimum van 20 wordt aangehouden.

\section{DEEL II - LINKSZIJDIGE DARMOBSTRUCTIES}

Op dit moment zijn zowel acute resectie als de beide brug tot electieve resectie aanpakken geaccepteerde behandelmethoden voor patiënten met linkszijdige obstruerende tumoren. Stent als brug tot electieve resectie is uitgebreid onderzocht en meerdere meta-analysen tonen significant meer laparoscopische resecties, minder complicaties, minder tijdelijke stoma's en meer primaire anastomosen wanneer stent wordt vergeleken met acute resectie. ${ }^{27}$ Hoewel dit veelbelovende uitkomsten zijn, heeft het prematuur sluiten van verschillende gerandomiseerde studies door een hoog aantal stent-gerelateerde complicaties en onzekerheden met betrekking tot de oncologische veiligheid vraagtekens opgeroepen over hoe veilig stentplaatsing daadwerkelijk is. ${ }^{28-31}$ Dit heeft tevens geleid tot een sterke reductie in het aantal stents dat geplaatst is de afgelopen jaren. ${ }^{32,33}$ Gedurende deze tijdsperiode heeft het aanleggen van een dubbelloops stoma als brug tot electieve resectie juist aan populariteit gewonnen. In theorie kan deze aanpak alle voordelen bieden van een brug tot electieve resectie aanpak, zonder het risico op stent-gerelateerde complicaties of directe tumor manipulatie. Onze volgende onderzoeksvraag is daarom;

Is het aanleggen van een dubbelloops colostoma als brug tot electieve resectie een veilig en goed alternatief voor acute resectie in patiënten met een linkszijdige obstruerende darmtumor? 
In hoofdstuk vijf wordt een systematische review gepresenteerd, waarin alle literatuur ten aanzien van deze vraag is geïdentificeerd. Acht vergelijkende studies, waarin een totaal van 2.424 patiënten werd mee genomen, laten geen significante verschillen in morbiditeit en mortaliteit zien. Belangrijk bij de interpretatie van deze uitkomsten is echter dat zes van de acht geïncludeerde studies gepubliceerd zijn tussen 1977 en 1995. Er zijn slechts twee recente studies. Gezien de ontwikkelingen in de afgelopen decennia in zowel de geneeskunde als de chirurgie, is de kans groot dat de uitkomsten van de oudere studies niet meer actueel zijn. Een evidente trend in de afname van het aantal postoperatieve sterfgevallen van $15 \% 30$ jaar geleden naar ongeveer $7 \%$ in meer recente studies, laat zien hoeveel invloed de ontwikkelingen in de gezondheidszorg hebben. ${ }^{32,34}$ Dit zien we ook terug in de studies geïncludeerd in hoofdstuk 5; de mortaliteit na stoma-aanleg en electieve resectie was 1,1\% en 3,7\% in de twee meest recente studies uit 2009 en 2013, vergeleken met een gemiddelde van 15,9\% in de oudere studies (1977-1995). Om meer up-to-date uitkomsten te verkrijgen voor stomaaanleg als brug tot electieve resectie, hebben wij een cohort studie van 146 patiënten verricht (hoofdstuk 6). Alle patiënten waarbij een dubbelloops stoma werd aangelegd voor een obstruerende linkszijdige tumor tussen 2003-2015 werden hierin geïncludeerd. 85 van deze 146 patiënten kregen het stoma als brug tot electieve resectie, waarbij uiteindelijk twee patiënten postoperatief zijn overleden (2,4\%). Deze $2,4 \%$ is vergelijkbaar met de $1,1 \%$ en $3,7 \%$ die werd gevonden in de andere twee recente studies, en lijkt lager dan de $7 \%$ mortaliteit die wordt gevonden in recente studies na acute resectie. ${ }^{32,35}$

Daarnaast lijkt stoma-aanleg als brug tot electieve resectie te leiden tot een hoger aantal laparoscopische resecties en primaire anastomosen (hoofdstuk 5 en 6)..$^{32}$ Ook het aantal permanente stoma's lijkt lager met $18 \%$ in hoofdstuk 6 , vergeleken met $35,2 \%$ in een van de meest recente meta-analyses waarin acute resectie wordt onderzocht. ${ }^{27}$ Een belangrijke reden voor behandelaren om af te zien van stoma-aanleg als brug tot electieve resectie, is de negatieve invloed die een stoma heeft op de kwaliteit van leven. ${ }^{36.37}$ Echter, de overgrote meerderheid van stoma's die als brug tot electieve resectie worden aangelegd, worden uiteindelijk opgeheven. Het aantal permanente stoma's na acute resectie is zelfs significant hoger. Dit zou dan ook geen reden moet zijn om deze behandeling niet toe te passen.

Hoewel sterk bewijs ontbreekt, wijst initiële data uit dat het aanleggen van een deviërend stoma als brug tot electieve resectie veilig is. Daarnaast lijkt het een goed alternatief voor acute resectie, gezien de veelbelovend lage morbiditeit en mortaliteitspercentages.

Colostoma-aanleg als brug tot electieve resectie lijkt dus een goed alternatief voor acute resectie. Het is echter ook belangrijk dat deze behandelaanpak vergeleken wordt met de andere brug tot electieve resectie mogelijkheid; stentplaatsing. Op dit moment bestaat er geen consensus over welke van deze twee beter is. ${ }^{32,33}$ Dit brengt ons naar de volgende onderzoeksvraag;

\section{Welke brug tot electieve resectie aanpak is beter; stentplaatsing of stoma aanleg?}

In de huidige richtlijnen wordt de voorkeur gegeven aan stentplaatsing boven stoma-aanleg. Stoma-aanleg wordt als eerste keus aanbevolen indien de obstructie langer dan $40 \mathrm{~mm}$ is, 
indien de endoscopist onvoldoende ervaring heeft met het plaatsen van stents of wanneer patiënten in de toekomst met angiogenese inhibitors behandeld zullen worden, aangezien dit bekende risicofactoren voor stentperforatie zijn..$^{25,38,39}$ De belangrijkste reden dat stentplaatsing wordt aangeraden boven stoma-aanleg, is omdat een stoma de kwaliteit van leven negatief beinvloedt. ${ }^{36,40}$ Deze aanbeveling is echter niet gebaseerd op wetenschappelijke data aangezien er toen de richtlijn werd opgesteld in 2014, geen studies bestonden die stent en stoma met elkaar vergeleken. Sinds 2014, zijn er twee studies gepubliceerd die acute resectie vergelijken met stentplaatsing en stoma-aanleg als brug tot electieve resectie. Daarnaast is er één studie verschenen die specifiek de twee brug tot electieve resectie aanpakken vergelijkt (hoofdstuk 7).

Tanis et al. hebben een grote populatiestudie verricht, waarbij 1.485 acute resectie patiënten zijn vergeleken met 196 stent en 135 stoma patiënten. Ostaimo et al. hebben een gelijkende studie verricht, hoewel met aanzienlijk minder patiënten. Het voornaamste doel van deze twee studies was het vergelijken van acute resectie met een brug tot electieve resectie aanpak. P-waarden voor stent versus stoma ontbreken hierdoor. Het mortaliteitspercentage in de studie van Tanis et al. was gelijkend in de stent $(5,6 \%)$ en stoma groep $(3,7 \%)$. Ook morbiditeit, het aantal laparoscopische resecties, het aantal primaire anastomosen en het aantal tijdelijke en permanente stoma's was gelijk. ${ }^{32,33}$ Deze conclusies zijn echter alleen gebaseerd op descriptieve data.

De eerste en enige studie die de brug tot electieve resectie methodes vergelijkt, laat andere resultaten zien (hoofdstuk 7). Hoewel mortaliteit, morbiditeit en opnameduur gelijk zijn, verschillen andere eindpunten. In de stomagroep is het aantal operatieve ingrepen en lange termijn complicaties significant hoger. Dit verschil kan worden verklaard doordat veel patiënten een derde operatieve ingreep moeten ondergaan om het stoma op te heffen. Daarnaast draagt de hoge incidentie van littekenbreuken op de oude stomalocatie bij, aangezien veel patiënten hiervoor een operatieve correctie moeten ondergaan. In de stentgroep bleken significant minder resecties laparoscopisch uitgevoerd te zijn.

Over het geheel lijken zowel stentplaatsing als stoma-aanleg effectieve methoden om de darm te decomprimeren. Stentplaatsing lijkt te leiden tot minder operatieve ingrepen en minder lange termijn complicaties. De consequenties van stent-gerelateerde perforaties kunnen echter ernstig zijn en het is vooralsnog niet duidelijk of het plaatsen van een stent oncologisch veilig is. ${ }^{41,42}$ In de keuze voor een bepaalde behandelaanpak, is met name het gesprek met de individuele patiënt daarom van zeer groot belang, waarbij gezamenlijk tot een beslissing moet worden gekomen. Met name patiënten die met curatieve insteek worden behandeld, kunnen terughoudend zijn in het accepteren van mogelijke oncologische gevolgen en daarom voor een stoma kiezen. Gezien het feit dat het aantal permanente stoma's in beide brug tot electieve resectiegroepen gelijk is, verwachten we geen significante impact op de kwaliteit van leven in de stomagroep. Patiënten moeten echter wel worden voorgelicht over de mogelijkheid van het ondergaan van meerdere interventies. 
Toekomstige, kwalitatief betere studies zijn noodzakelijk om uit te wijzen welke brug tot electieve resectie aanpak de voorkeur verdient. Op dit moment werken wij aan een propensityscore gematchte analyse waarbij we stent met stoma vergelijken. Patiënten zullen worden geselecteerd vanuit een grote nationale database, welke wij afgelopen jaar hebben samengesteld. Hierin zijn alle patiënten opgenomen die zich tussen 2009 en 2016 in Nederland met een obstructieve tumor in het linkszijdige colon hebben gepresenteerd. Gezien het gebrek aan data van gerandomiseerde studies, zal deze quasi-gerandomiseerde studie veel bijdragen aan de huidige kennis.

Zoals al eerder is aangegeven, verlaagt een permanent stoma de kwaliteit van leven. ${ }^{36,37}$ Dit is een belangrijk gegeven, aangezien veel patiënten met darmkanker geconfronteerd zullen worden met een stoma gedurende het behandeltraject. Met name patiënten die zich presenteren met een acute darmobstructie ten gevolge van een tumor, hebben een hoge kans op stoma-aanleg. ${ }^{7,43} \mathrm{Er}$ bestaan verschillende typen stoma's, waarbij het op dit moment onduidelijk is welk type het beste is. Wanneer een (in opzet) tijdelijk stoma wordt aangelegd, kan de chirurg kiezen tussen het aanleggen van een dubbelloops ileostoma (dunne darm stoma) of colostoma (dikke darm stoma). Het is belangrijk dat het meest optimale type stoma wordt aangelegd, zodat de kwaliteit van leven zo min mogelijk wordt aangetast. Er bestaat op dit moment echter geen consensus over welk type stoma beter is. De onderzoeksvraag die hieruit voort komt is dan ook;

\section{Welk type stoma heeft de voorkeur; een ileostoma of colostoma?}

Met name de indicatie voor stoma-aanleg bepaalt welk type geïndiceerd is. Patiënten waarbij een stoma wordt aangelegd voor een linkszijdige obstruerende tumor, zijn immers niet geholpen met een ileostoma doordat de klep op de dunne-dikke darmovergang adequate decompressie verhinderd. Dit kan leiden tot levensbedreigende aandoeningen zoals een darmperforatie, en moet daarom ten alle tijden voorkomen worden. Een obstruerende tumor is echter slechts in de minderheid van de gevallen de onderliggende reden om een stoma aan te leggen. De meest voorkomende indicatie is ter bescherming van een nieuw aangelegde darmnaad. ${ }^{44}$ Voor deze, en eigenlijk elke andere indicaties, kan zowel een ileo- als colostoma aangelegd worden. Hoewel er geen richtlijn bestaat die een aanbeveling doet op dit gebied, lijkt in recente literatuur de voorkeur uit te gaan naar een ileostoma (hoofdstuk 8). Patiënten met een ileostoma hebben minder wondinfecties, minder vaak een stomaprolaps, stoma retracties, parastomale hernia's en littekenbreuken. Daarnaast rapporteren zij een betere kwaliteit van leven. Het hoge risico op een parastomale hernia bij een colostoma is in een zeer recente studie met meer dan 5.000 patiënten nogmaals bewezen.$^{45}$ Een belangrijke overweging is echter dat patiënten met een ileostoma een significant hoger risico hebben op de ontwikkeling van een high-output stoma. Dit kan dehydratie en zelfs nierfalen tot gevolg hebben. Hoewel nierfalen een zeer zeldzame complicatie is, is deze wel ernstig en mogelijk levensbedreigend. We raden aanleg van een ileostoma daarom niet aan bij patiënten die reeds een pre-existent verhoogd risico hebben op de ontwikkeling van een high-output stoma, 
dehydratie of nierfalen. Hierbij gaat het bijvoorbeeld om patiënten die niet goed in staat zijn om voor zichzelf te zorgen; zoals patiënten met mentale retardatie, dementie of patiënten die bed gebonden zijn. Ook patiënten met pre-existent een slechte nierfunctie hebben een hoger risico op het ontwikkelen van complicaties wanneer een high-output stoma optreedt. ${ }^{46}$ Als laatste zijn er ook aanwijzingen dat chemotherapie, in het specifiek capecitabine, een verhoogde kans geven op diarree en daarmee een high-output stoma. ${ }^{47,48}$ De keuze of een ileo- of colostoma wordt aangelegd moet per patiënt worden bekeken. Een behulpzame flowchart kunt $\mathrm{u}$ terug vinden in hoofdstuk 8.

\section{DEEL III - LANGE TERMIJN UITKOMSTEN}

De afgelopen jaren zijn er steeds meer studies verschenen die de gunstige korte termijn uitkomsten na een brug tot electieve resectie aanpak bevestigen. Zowel stentplaatsing als stoma aanleg leiden tot meer laparoscopische resecties, meer primaire anastomosen, minder (tijdelijke) stoma's en mogelijk ook minder complicaties (hoofdstuk 5-7). ${ }^{27}$

$\mathrm{Er}$ is echter weinig bewijs ten aanzien van de lange termijn uitkomsten. Met name voor stentplaatsing is dit relevant, gezien de vraagtekens die zijn gezet bij de oncologische veiligheid. ${ }^{41}$ Verschillende studies laten nadelige verandering in de pathologische uitkomsten zien na stentplaatsing. Zo lijkt het aantal circulerende tumorcellen in het bloed te verhogen na stentplaatsing en wordt er vaker peri-neurale en lymfevatinvasie gezien in de preparaten. ${ }^{41,49-52}$ Hoewel het onbekend is in hoeverre deze surrogaat uitkomstmaat ook daadwerkelijk klinisch relevant is, blijft de onzekerheid over oncologische veiligheid na stentplaatsing een belangrijke reden waarom deze behandelstrategie weinig wordt toegepast in curatieve patiënten. Dit heeft ons gebracht tot de volgende onderzoeksvraag;

\section{Is stentplaatsing oncologisch veilig in patiënten die met curatieve opzet worden behandeld?}

In eerdere studies naar de veiligheid van stentplaatsing lag de focus op korte termijn uitkomsten, waarbij meerdere gerandomiseerde studies vroegtijdig zijn afgebroken door een hoog aantal complicaties in de stentgroep. ${ }^{42}$ De lange termijn resultaten die op dit moment beschikbaar zijn, zijn daardoor voornamelijk afkomstig van retrospectieve cohort studies. Twee meta-analyses uit 2015 en 2017, waarin respectievelijk 11 en 17 studies zijn geïncludeerd, vonden geen verschil in overleving of ziekte vrije overleving. ${ }^{53,54}$ Vanwege het toenemende aantal studies over de lange termijn uitkomsten na stentplaatsing, kunt $u$ in hoofdstuk 9 een ge-update meta-analyse vinden. Hierbij zijn de resultaten van 21 studies samengevoegd, waarbij er opnieuw geen verschil wordt gevonden in overleving, ziekte vrije overleving en het aantal lokale recidieven tussen de stent en acute resectie groep. 16 van de 21 studies zijn echter retrospectief en de resultaten kunnen daardoor zijn beïnvloed door selectiebias. Het feit dat de sensitiviteitsanalyse tegenstrijdige uitkomsten laat zien, met een trend naar een lagere overleving in de stentgroep wanneer alleen de gerandomiseerde studies worden mee genomen, sterkt dit vermoeden. Studies van meer kwaliteit zijn daarom noodzakelijk om definitieve conclusies te trekken. In hoofdstuk 10 hebben we daarom 222 stent patiënten 
gematched met 444 acute resectie patiënten op basis van hun propensity score, waarmee we een quasi-gerandomiseerd design hebben gecreëerd. Verschillen tussen de beide behandelgroepen waren al laag voor matching, doordat stents in Nederland vrijwel alleen worden geplaatst in gespecialiseerde centra, waarbij behandeltoewijzing niet selectief is. Matchen op basis van propensity score heeft selectiebias nog verder geminimaliseerd. Ook in deze studie vonden we geen verschil in de lange termijn oncologische uitkomsten. Kang et al. hebben een gelijkende studie verricht, waarbij 109 acute resectie patiënten zijn vergeleken met 226 stent patiënten, zij hebben de propensity score gebruikt als corrigerende factor in hun analyse. Ook zij vonden geen verschil in uitkomsten. ${ }^{55}$ Hoewel data van gerandomiseerde studies ontbreekt, zijn de laatste twee genoemde studies van beduidend betere kwaliteit dan de eerder gepubliceerde studies. Het wordt hierdoor dus steeds aannemelijker dat stentplaatsing oncologisch veilig is.

Twee recente meta-analysen laten zien dat een toename in het aantal circulerende tumorcellen in het bloed significant geassocieerd is met een slechtere prognose. ${ }^{56,57}$ Het direct toepassen van deze data naar stentplaatsing lijkt echter te kort door de bocht. Een verhoging van het aantal circulerende tumorcellen na stentplaatsing is oncologisch gezien mogelijk niet relevant, aangezien het waarschijnlijk een tijdelijk en voorbijgaand fenomeen is. Daarnaast zien we ook een stijging in het aantal circulerende tumorcellen postoperatief, welke normaliseert na verloop van tijd zonder dat dit oncologische gevolgen heeft. ${ }^{58}$ Wind et al., hebben daarnaast laten zien dat het aantal circulerende tumorcellen significant meer toeneemt na een open resectie versus een laparoscopische resectie. ${ }^{59}$ Aangezien een laparoscopische resectie significant vaker kan worden uitgevoerd in de stentgroep, zou dit ook tegenwicht kunnen geven voor de initiële verhoging in circulerende tumorcellen veroorzaakt door stentplaatsing. Op dit moment is het nog onduidelijk wat de invloed is van een stijging in circulerende tumorcellen na een interventie. Conclusies ten aanzien van de overleving moeten derhalve niet enkel worden gebaseerd op surrogaat uitkomsten.

Hoewel steeds meer bewijs lijkt uit te wijzen dat ongecompliceerde stentplaatsing oncologisch veilig is, zijn stent-gerelateerde perforaties duidelijk geassocieerd met meer lokale recidieven en mogelijk zelfs een slechtere overlevingskans (hoofdstuk 10). ${ }^{60,61}$ Als we echter kijken naar de resultaten van Kang et al. en de resultaten van hoofdstuk 10, dan lijkt dit geen invloed te hebben op de oncologische uitkomsten van de gehele stentgroep..$^{55}$ De lagere overlevingskans in de patiënten subgroep die een perforatie krijgt, lijkt uitgebalanceerd te worden door een positieve impact van stentplaatsing in andere subgroepen. Zo zijn er bijvoorbeeld studies die laten zien dat postoperatieve complicaties de oncologische uitkomsten ook negatief kunnen beïnvloeden. ${ }^{63}$ Aangezien stent-gerelateerde perforaties de oncologische uitkomsten negatief lijken te beïnvloeden, is het wel van uiterst belang dat het optreden hiervan zo veel mogelijk voorkomen wordt. Van belang hierbij is dat de stents altijd geplaatst worden door een ervaren endoscopist. Daarnaast moet stentplaatsing worden vermeden in patiënten die een goedaardige darmobstructie hebben, of in patiënten die in de toekomst bevacizumab zullen krijgen, aangezien dit bekende risicofactoren zijn. ${ }^{63,64}$ Verder moet post-stent dilatatie ten alle tijden vermeden worden. ${ }^{64}$ 
Per individuele patiënt zullen de voordelen en risico's van elke behandelstrategie afgewogen moeten worden om een weloverwogen beslissing te kunnen maken. Een jonge, gezonde patiënt met een laag operatierisico zal waarschijnlijk eerder kiezen voor acute resectie, om het risico op een stent-perforatie te vermijden. Bij oudere patiënten met significante comorbiditeit kan het $8 \%$ perforatie risico echter in het niet vallen bij het operatie risico. Daarnaast moet met de patiënt besproken worden wat het risico is op een permanent stoma, dit is immers significant lager in de brug tot electieve resectiegroepen.

Hoewel het aanleggen van een stoma als brug tot electieve resectie geen invloed heeft op de oncologische uitkomsten, wordt er wel gesuggereerd dat deze behandelaanpak leidt tot een verhoogd aantal chirurgische ingrepen. Naast dat het stoma gedurende een derde ingreep moet worden opgeheven, spelen littekenbreuken hier ook een grote rol bij. Om dit goed te onderzoeken hebben we de volgende onderzoeksvraag opgesteld:

\section{Wat is het risico op het ontwikkelen van een littekenbreuk na stoma-ophef?}

Enkele oudere studies hebben de littekenbreuk incidentie geschat op 7\% na stoma-ophef. ${ }^{65-67}$ Meer recente studies laten echter veel hogere getallen zien met een incidentie tot $35 \% .{ }^{68-70}$ In een recent cohortonderzoek, waarbij 318 opeenvolgende patiënten werden onderzocht die stoma-ophef hadden ondergaan, bleek $34,6 \%$ uiteindelijk een littekenbreuk te hebben in het stomalitteken. In $65,5 \%$ van deze patiënten waren de klachten dusdanig, dat een operatieve correctie nodig was (hoofdstuk 11).

De daadwerkelijke incidentie van littekenbreuken na stoma-aanleg lijkt rond de $35 \%$ te liggen. Dit is aanzienlijk hoger dan de incidentie die wordt gerapporteerd na laparotomie incisies, waar het percentage rond de 10 procent ligt. ${ }^{71,72}$ Dit heeft ertoe geleid dat sommigen vinden dat stoma-ophef primair moet worden gezien als littekenbreukcorrectie, in plaats van slechts een fasciesluiting. ${ }^{73}$ Dit impliceert dat (profylactische) mesh plaatsing nodig is, om littekenbreuken in de toekomst te voorkomen. Er bestaan enkele retrospectieve studies waarin dit wordt onderzocht, waarin veelbelovende resultaten worden getoond. ${ }^{69,74}$ Een belangrijke reden dat artsen terughoudend zijn met het plaatsen van een preventieve mesh bij stomaophef, is dat dit gebied mogelijk gecontamineerd is en hierdoor een hoger risico geeft op infectieuze complicaties. Daarom is het van belang om specifiek die patiënten te identificeren die een hoog risico hebben op het ontwikkelen van een littekenbreuk, aangezien zij het meeste baat zouden kunnen hebben bij preventieve maatregleen, zoals mesh plaatsing.

Bekende risicofactoren voor het ontwikkelen van een littekenbreuk na stoma-ophef zijn een BMI boven de 25, het hebben van een colostoma (versus een ileostoma), hypertensie, diabetes mellitus en het hebben van een stomaprolaps of parasomale hernia (hoofdstuk 11).69,71,75,76 Preventieve mesh plaatsing in deze patiënten kan de littekenbreukincidentie aanzienlijk verminderen en hiermee ook het aantal chirurgische ingrepen. ${ }^{77}$ Op dit moment zijn er nog geen gerandomiseerde studies beschikbaar die dit onderzoeken. Na de publicatie van 
hoofdstuk 11, hebben wij besloten om dit zelf te onderzoeken middels de PRINCE studie. Dit is een gerandomiseerde studie waarbij alle patiënten die stoma-ophef ondergaan worden geïncludeerd. Patiënten worden hierna gerandomiseerd naar conventionele stomaophef of met profylactische mesh plaatsing. Hopelijk zullen de uitkomsten van deze studie in de toekomst uitwijzen of het preventief plaatsen van een mesh tijdens stoma-ophef veilig en voordelig is.

\section{CONCLUSIE}

Hoewel de incidentie van obstruerende darmkanker waarschijnlijk afgenomen is na invoering van het bevolkingsonderzoek enkele jaren geleden, presenteert nog altijd $8 \%$ van de patiënten zich met een acute kwaadaardige darmobstructie. ${ }^{11}$ In dit proefschrift worden meerdere dilemma's ten aanzien van de behandeling van obstructieve darmtumoren besproken. Het is van groot belang dat er per individuele patiënt wordt gekeken welke behandeling het beste is, waarbij de patiënt uitgebreid moet worden voorgelicht zodat deze actief mee kan denken bij het maken van een beslissing. De lage morbiditeit en mortaliteit onder jonge ( $<70$ jaar) en gezonde (ASA 1/2) patiënten verantwoordt het toepassen van een acute resectie. Een belangrijke afweging hierbij is echter wel dat het aantal permanente stoma's na een acute resectie hoger is dan na een brug tot electieve resectie aanpak, ook in de jongere leeftijdscategorie. De patiënt moet hierover ingelicht worden, voordat wordt besloten welke behandeling wordt toegepast. Stentplaatsing kan een goede optie zijn voor patiënten die pertinent geen stoma willen, met name aangezien er steeds meer bewijs komt dat deze methode ook oncologisch veilig is. Hierbij raden wij stentplaatsing echter alleen aan indien er voldoende ervaring is, gezien het feit dat stent-gerelateerde perforaties ernstige gevolgen kunnen hebben, ook op de lange termijn.

In oudere patiënten met co-morbiditeit raden wij altijd een brug tot electieve resectie aanpak te kiezen. Dit leidt in deze patiënten niet alleen tot significant minder permanente stoma's, maar ook minder complicaties en een lagere postoperatieve mortaliteit. Of hierbij een stent moet worden geplaatst of een stoma moet worden aangelegd, moet per individuele patiënt bekeken en besproken worden. Stentplaatsing moet echter alleen aangeboden worden als er voldoende ervaring is. Indien dit niet het geval is, dan geven wij de voorkeur aan stoma aanleg. 


\section{REFERENTIES}

1. World Cancer Research Fund International (WCRF). Worldwide cancer registry. https://www.wcrf.org/

2. The Netherlands Comprehensive Cancer Organisation (IKNL). The Netherlands Cancer Registry. http://www. cijfersoverkanker.nl/

3. Astin M, Griffin T, Neal RD, Rose P, Hamilton W. The diagnostic value of symptoms for colorectal cancer in primary care: a systematic review. BJGP. 2011;61(586):e231-e243

4. Jullumstro E, Wibe A, Lydersen S, Edna TH. Colon cancer incidence, presentation, treatment and outcomes over 25 years. Colorectal Dis 2011;13(5):512-518

5. Scott NA, Jeacock J, Kingston RD. Risk factors in patients presenting as an emergency with colorectal cancer. BJS 1995;82(3):321-3

6. Hennessey DB, Burke JP, Ni-Dhonochu T, Shields C, Winter DC, Mealy K. Preoperative hypoalbuminemia is an independent risk factor for the development of surgical site infection following gastrointestinal surgery: a multi-institutional study. Ann Surg 2010;252(2):325-9

7. Sjo OH, Larsen S, Lunde OC, Nesbakken A. Short term outcome after emergency and elective surgery for colon cancer. Colorectal Dis. 2009;11:733-739

8. Papadimitriou G, Manganas D, Phedias Georgiades C, Vougas V, Vardas K, Drakopoulos S. Emergency surgery for obstructing colorectal malignancy: prognostic and risk factors. J BUON. 2015;20(2):406-12

9. Smothers L, Hynan L, Fleming J, Turnage R, Simmang C, Anthony T. Emergency surgery for colon carcinoma. DCR 2003;46(1):24-30

10. Iversen LH, Bülow S, Christensen IJ, Laurberg S, Harling $\mathrm{H}$. Postoperative medical complications are the main cause of early death after emergency surgery for colonic cancer. BJS. 2008;95:1012-9

11. Tanis PJ, Paulino Pereira NR, van Hooft JE, Consten EC, Bemelman WA. Resection of obstructive left-sided colon cancer at a national level: a prospective analysis of short-term outcomes in 1,816 patients. Dig Surg 2015;32(5):317-324

12. Phillips RKS, Hittinger R, Fry JS, Fielding LP. Malignant large bowel obstruction. Br J Surg. 1985;72:296-302

13. Bonin EA, Baron TH. Update on the Indications and Use of Colonic Stents. Curr Gastroenterol Rep 2010 12(5):374-382
14. Tan KK, Sim R. Surgery for obstructed colorectal malignancy in an Asian population: predictors of morbidity and comparison between left- and right-sided cancers. J Gastrointest Surg 2010;14:295

15. Kye BH, Lee YS, Cho HM, Kim JG, Oh ST, Lee IK, Kang WK, Ahn CH5, Lee SC, Park JK, Kim HJ8. Comparison of LongTerm Outcomes Between Emergency Surgery and Bridge to Surgery for Malignant Obstruction in RightSided Colon Cancer: A Multicenter Retrospective Study. Ann Surg Oncol. 2016;23(6):1867-74

16. Ji WB, Kwak JM, Kang DW, Kwak HD, Um JW, Lee SI, Min BW, Sung NS, Kim J, Kim SH.Clinical benefits and oncologic equivalence of self-expandable metallic stent insertion for right-sided malignant colonic obstruction. Surg Endosc. 2017;31(1):153-158

17. Keymling M. Colorectal stenting. Endoscopy. 2003;35:234-238

18. Bhardwaj R, Parker MC. Palliative therapy of colorectal carcinoma: stent or surgery? Colorectal Dis. 2003;5:518-521

19. Stimac D. Colonic stents for the palliation of malignant colonic obstruction. Dig Dis. 2008;26:336-341

20. Cho YK, Kim SW, Lee BI, et al. Clinical outcome of selfexpandable metal stent placement in the management of malignant proximal colon obstruction. Gut Liver. 2011;5:165-70

21. Schoonbeek PK1, Genzel P, van den Berg EH, van Dobbenburgh OA, Ter Borg. .Outcomes of SelfExpanding Metal Stents in Malignant Colonic Obstruction are Independent of Location or Length of the Stenosis: Results of a Retrospective, Single-Center Series. Dig Surg. 2018;35(3):230-235

22. Small AJ, Coelho-Prabhu N, Baron TH. Endoscopic placement of self-expandable metal stents for malignant colonic obstruction: long-term outcomes and complication factors. Gastrointest Endosc 2010;71(3):560-572

23. Lee HJ, Park SJ, Cheon JH, Kim TI, Kim WH, Hong SP. What is the necessity of endoscopist for successful endoscopic stenting in patients with malignant colorectal obstruction? Int J Colorectal Dis. 2015;30(1):119-25

24. Williams,D.; Law,R.; Pullyblank,A.M. Colorectal stenting in malignant large bowel obstruction: the learning curve. Int J Surg Oncol., 2011:9:17848

25. van Hooft JE, van Halsema EE, Vanbiervliet $G$ et al. Selfexpandable metal stents for obstructing colonic and 
extracolonic cancer: European Society of Gastrointestinal Endoscopy (ESGE) Clinical Guideline. Endoscopy. 2014;46(11):990-1053

26. Mege D, Manceau G, Beyer-Berjot L, Bridoux V, Lakkis Z, Venara A, Voron T, Brunetti F, Sielezneff I, Karoui M; AFC (French Surgical Association) Working Group. Surgical management of obstructive right-sided colon cancer at a national level results of a multicenter study of the French Surgical Association in 776 patients. Eur J Surg Oncol. 2018;6;S0748-7983(18)31171-5

27. Arezzo A, Passera R, Lo Secco G, Verra M, Bonino MA, Targarona $E$, Morino M. Stent as bridge to surgery for left-sided malignant colonic obstruction reduces adverse events and stoma rate compared with emergency surgery: results of a systematic review and meta-analysis of randomized controlled trials. Gastrointest Endosc. 2017; 86:416-426

28. J.E. van Hooft, W.A. Bemelman, B. Oldenburg, A.W. Marinelli, M.F. Holzik, M.J. Grubben, M.A. Sprangers, M.G. Dijkgraaf, P. Fockens, Colonic stenting versus emergency surgery for acute left-sided malignant colonic obstruction: a multicentre randomised trial, Lancet Oncol. 2011;12;344e352

29. H.Y. Cheung, C.C. Chung, W.W. Tsang, J.C. Wong, K.K. Yau, M.K. Li, Endolaparoscopic approach vs conventional open surgery in the treatment of obstructing left-sided colon cancer: a randomized controlled trial, Arch. Surg. 2009;144;12;1127e1132

30. L.A. Pirlet, K. Slim, F. Kwiatkowski, F. Michot, B.L. Millat, Emergency preoperative stenting versus surgery for acute left-sided malignant colonic obstruction: a multicenter randomized controlled trial, Surg. Endosc. $2011 ; 25 ; 1814$ e1821

31. C. Sabbagh, F. Browet, M. Diouf, et al., Is stenting as "a Bridge to Surgery" an oncologically safe strategy for the management of acute, left-sided, malignant, colonic obstruction?: a comparative study with a propensity score analysis, Ann. Surg. 2013;258;107e115

32. Tanis PJ, Paulino Pereira NR, van Hooft JE, Consten EC, Bemelman WA. Resection of obstructive left-sided colon cancer at a national level: a prospective analysis of short-term outcomes in 1,816 patients. Dig Surg 2015;32(5):317-324

33. Öistämö E, Hjern F, Blomqvist L, Falkén Y, Pekkari K, Abraham-Nordling M. Emergency management with resection versus proximal stoma or stent treatment and planned resection in malignant left-sided colon obstruction. World Journal of Surgical Oncology. 2016;14(1):232

34. Tekkis PP, Kinsman R, Thompson MR, et al. The Association of Coloproctology of Great Britain and Ireland study of large bowel obstruction caused by colorectal cancer. Ann Surg 2004;240:76-81

35. J.K. Jiang, Y.T. Lan, T.C. Lin, W.S. Chen, S.H. Yang, H.S Wang, S.C. Chang, J.K. Lin, Primary vs. delayed resection for obstructive left-sided colorectal cancer: impact of surgery on patient outcome, Dis. Colon Rectum 2008;51(3)06e311

36. Young CJ, De-Loyde KJ, Young JM, Solomon MJ, Chew $\mathrm{EH}$, Byrne CM, Salkeld G, Faragher IG Improving quality of life for people with incurable large-bowe obstruction: randomized control trial of colonic stent insertion. Dis Colon Rectum 2015;58(9):838-849

37. Sprangers MA, Taal BG, Aaronson NK, te Velde A. Quality of life in colorectal cancer. Stoma vs. nonstoma patients. Dis Colon Rectum 1995;38(4):361-369

38. van Halsema EE, van Hooft JE, Small AJ, Baron TH, García-Cano J, Cheon JH, Lee MS, Kwon SH, MucciHennekinne S, Fockens P, Dijkgraaf MG, Repici A. Perforation in colorectal stenting: a meta-analysis and a search for risk factors. Gastrointest Endosc. 2014;79(6):970-82.e7

39. Boyle DJ, Thorn C, Saini A, Elton C, Atkin GK, Mitchell IC, Lotzof K, Marcus A, Mathur P. Predictive factors for successful colonic stenting in acute large-bowe obstruction: a 15-year cohort analysis. Dis Colon Rectum. 2015;58(3):358-62

40. Gandrup P, Lund L, Balslev I. Surgical treatment of acute malignant large bowel obstruction. Eur J Surg 1992 158: $427-430$

41. Sabbagh C, Browet F, Diouf M et al. Is stenting as "a bridge to surgery" an oncologically safe strategy for the management of acute, left-sided, malignant, colonic obstruction? A comparative study with a propensity score analysis. Ann Surg 2013;258:107-115

42. van Hooft JE, Bemelman WA, Oldenburg B et al. Colonic stenting versus emergency surgery for acute left-sided malignant colonic obstruction: a multicentre randomised trial. Lancet Oncol. 2011;12(4):344-52

43. Bayar B, Yılmaz KB, Akıncı M, Şahin A, Kulaçoğlu H. An evaluation of treatment results of emergency versus elective surgery in colorectal cancer patients. Turkish Journal of Surgery/Ulusal cerrahi dergisi. 2016;32(1):1117

44. Scott-Conner, CEH. Chassin's operative strategy in colon and rectal surgery. New York: Springer; 2006:18

45. Andersen RM, Klausen TW, Danielsen AK, Vinther A, Gögenur I, Thomsen T. Incidence and risk factors for parastomal bulging in patients with ileostomy or colostomy: a register-based study using data from the Danish Stoma Database Capital Region. Colorectal Dis 2018;20(4):331-340 
46. Messaris E, Sehgal R, Deiling $S$, et al. Dehydration is the most common indication for readmission after diverting ileostomy creation. Dis Colon Rectum. 2012;55:175-80

47. Robertson JP, Wells CI, Vather R, Bissett IP. Effect of Diversion lleostomy on the Occurrence and Consequences of Chemotherapy-Induced Diarrhea. Dis Colon Rectum. 2016;59:194-200

48. Oliphant R, Czerniewski A, Robertson I, McNulty C, Waterston A, Macdonald A. The effect of adjuvant chemotherapy on stoma-related complications after surgery for colorectal cancer: a retrospective analysis. J Wound Ostomy Continence Nurs. 2015;42:494-8

49. Maruthachalam K, Lash GE, Shenton BK, Horgan AF. Tumour cell dissemination following endoscopic stent insertion. Br J Surg 2007;94:1151-1154

50. Kim HJ, Choi GS, Park JS et al. Higher rate of perineural invasion in stent-laparoscopic approach in comparison to emergent open resection for obstructing left-sided colon cancer. Int J Colorect Dis 2013;28:207-14

51. Poon J. Pang R. Law W. The impact of colonic stenting on tumor cell dissemination in colorectal cancer patients. Dis.Colon Rectum 2011;54:160

52. Yamashita S, Tanemura M, Sawada G et al K. Impact of endoscopic stent insertion on detection of viable circulating tumor cells from obstructive colorectal cancer. Oncol Lett. 2018;15(1):400-406

53. Matsuda A, Miyashita M, Matsumoto S et al. Comparison of long-term outcomes of colonic stent as "bridge to surgery" and emergency surgery for malignant large-bowel obstruction: a meta-analysis. Ann Surg Oncol 2015;22(2):497-504

54. Ceresoli M, Allievi N, Coccolini F et al. Long-term oncologic outcomes of stent as a bridge to surgery versus emergency surgery in malignant left side colonic obstructions: a meta-analysis. J Gastrointest Oncol 2017;8(5):867-876

55. Kang SI, Oh HK, Yoo JS et al. Oncologic outcomes of preoperative stent insertion first versus immediate surgery for obstructing left-sided colorectal cancer. Surg Oncol. 2018;27(2):216-224

56. Tan $Y$, Wu H.The significant prognostic value of circulating tumor cells in colorectal cancer: A systematic review and meta-analysis. Curr Probl Cancer. 2017;29:S0147-272(17)30129-0

57. Yang C, Zou K, Zheng L, Xiong B. Prognostic and clinicopathological significance of circulating tumor cells detected by RT-PCR in non-metastatic colorectal cancer: a meta-analysis and systematic review. BMC Cancer. 2017;717-725.
58. Kaifi JT, Li G, Clawson G, Kimchi ET, Staveley-O'Carroll KF. Perioperative circulating tumor cell detection: Current perspectives. Cancer Biology \& Therapy. 2016;17(8):859-869

59. Wind J, Tuynman JB, Tibbe AG, Swennenhuis JF, Richel DJ, van Berge Henegouwen MI, Bemelman WA. Circulating tumour cells during laparoscopic and open surgery for primary colonic cancer in portal and peripheral blood. Eur J Surg Oncol. 2009;35(9):942-50

60. Sloothaak DA, van den Berg MW, Dijkgraaf MG, Fockens P, Tanis PJ, van Hooft JE, Bemelman WA; collaborative Dutch Stent-In study group. Oncological outcome of malignant colonic obstruction in the Dutch Stent-In 2 trial. BJS. 2014;101(13):1751-7

61. Avlund TH, Erichsen R, Ravn S, Ciplys Z, Andersen JC, Laurberg $S$, Iversen LH.The prognostic impact of bowel perforation following self-expanding metal stent as a bridge to surgery in colorectal cancer obstruction. Surg Endosc. 2018;32(1):328-336

62. Ha GW, Kim JH, Lee MR. Oncologic Impact of Anastomotic Leakage Following Colorectal Cancer Surgery: A Systematic Review and Meta-Analysis. Ann Surg Oncol. 2017;24(11):3289-3299

63. Small AJ, Coelho-Prabhu N, Baron TH. Endoscopic placement of self-expandable metal stents for malignant colonic obstruction: long-term outcomes and complication factors. Gastrointest Endosc 2010;71(3):560-572

64. van Halsema EE, van Hooft JE, Small AJ et al. Perforation in colorectal stenting: a meta-analysis and a search for risk factors. Gastrointest Endosc 2014;79(6):970-82

65. Tilney HS, Sains PS, Lovegrove RE, Reese GE, Heriot AG, Tekkis PP. Comparison of outcomes following ileostomy versus colostomy for defunctioning colorectal anastomoses. World J Surg 2007; 31: 1142-1151

66. Bakx R, Busch OR, BemelmanWA, Veldink GJ, Slors JF, van Lanschot JJ. Morbidity of temporary loop ileostomies. Dig Surg 2004; 21: 277-281

67. Guenaga KF, Lustosa SA, Saad SS, Saconato H, Matos D. lleostomy or colostomy for temporary decompression of colorectal anastomosis. Cochrane Database Syst Rev 2007; (1)CD004647

68. Bhangu A, Nepogodiev D, Futaba K. Systematic review and meta-analysis of the incidence of incisional hernia at the site of stoma closure. World J Surg 2012; 36: 973-983

69. Bhangu A, Fletcher L, Kingdon S, Smith E, Nepogodiev $D$, Janjua U. A clinical and radiological assessment of incisional hernias following closure of temporary stomas. Surgeon 2012; 10: 321-325 
70. Schreinemacher MH, Vijgen GH, Dagnelie PC, Bloemen JG, Huizinga BF, Bouvy ND. Incisional hernias in temporary stoma wounds

71. Kuhry E, Schwenk WF, Gaupset R, Romild U, Bonjer HJ. Long-term results of laparoscopic colorectal cancer resection. Cochrane Database Syst Rev 2008; (2) CD003432

72. Kingsnorth A, LeBlanc K. Hernias: inguinal and incisional. Lancet 2003; 362: 1561-1571.

73. Cingi A, Solmaz A, AttaallahW, Aslan A, Aktan AO. Enterostomy closure site hernias: a clinical and ultrasonographic evaluation. Hernia 2008; 12: 401-405
74. Maggiori L, Moszkowicz D, Zappa M, Mongin C, Panis Y. Bioprosthetic mesh reinforcement during temporary stoma closure decreases the rate of incisional hernia: a blinded, case-matched study in 94 patients with rectal cancer. Surgery 2015; 158: 1651-1657

75. Van Ramshorst GH, Nieuwenhuizen J, HopWC. Abdominal wound dehiscence in adults: development and validation of a risk model. World J Surg 2010; 34: 20-27

76. Sharp SP, Francis JK, Valerian BT, Canete JJ, Chismark $A D$, Lee EC. Incidence of ostomy site incisional hernias after stoma closure. Am Surg 2015; 81: 1244-1248

77. Warren JA, Beffa LR, Carbonell AM, Cull J, Sinopoli B, Ewing JA, McFadden C, Crockett J, Cobb WS. Prophylactic placement of permanent synthetic mesh at the time of ostomy closure prevents formation of incisional hernias. Surgery. 2018;163(4):839-846 


\section{ACKNOWLEDGEMENTS | DANKWOORD}

Eindelijk is het zo ver. Hij is af. En wat ben ik trots. Maar ik heb dit zeker niet alleen gedaan; dit proefschrift was er nooit geweest zonder de hulp van vele anderen die ik hier graag wil bedanken!

Geachte prof. Broeders, Beste Ivo, jij bent de rust zelve. En dat is een hele fijne eigenschap om te hebben in een promotor. $\mathrm{Na}$ een overleg of brainstorm sessie met jou had ik altijd het idee dat er weer structuur in mijn proefschrift kwam. Hoewel ik het liefst overal ja tegen zeg en alles tegelijk doe, heb je me geleerd dat selectiviteit een belangrijk onderdeel is van kwaliteit. Het feit dat de artikelen in dit boekje nu naadloos op elkaar afgestemd zijn, heb ik dan ook grotendeels aan jou te danken. Naast alles wat ik de afgelopen jaren van jou heb meegekregen, blij ik erg blij dat ik ook nog wat aan jouw algemene kennis bij heb mogen dragen. Het is immers enorm belangrijk om te beseffen wat voor enorme mijlpaal het is als je kids straks "exclusive" zijn!

Geachte prof. Siersema, als student geneeskunde heeft u me een platform gegeven tijdens het begin van mijn promotietraject. $U$ steunde me altijd in mijn onderzoeks-ideeën en dacht actief mee. Toen ik mijn wilde plannen over het meten van circulerende tumor cellen na stentplaatsing voorlegde, heeft u zelfs via contacten geholpen om uit te zoeken of het praktisch mogelijk was om dit in Nederland te doen. Hoewel dit project helaas niet van de grond is gekomen, zijn alle andere projecten meer dan geslaagd! Mede dankzij uw hulp heb ik daarnaast mijn onderzoek in San Diego bij de DDW kunnen presenteren, wat een onvergetelijke ervaring was dat. Heel erg bedankt!

Beste Esther, door jou ben ik in het Meander terecht gekomen en wat ben ik je daar dankbaar voor! Als $3^{\mathrm{e}}$-jaars geneeskunde student bood jij mij de kans om onderzoek te komen doen wat uiteindelijk heeft geresulteerd in dit proefschrift. Jouw continue begeleiding de afgelopen jaren heeft mij gevormd tot de wetenschapper en arts die ik nu ben. Met je eindeloze energie, inzet, extreme multi-tasking skills en overduidelijke passie voor het vak ben je een groot voorbeeld. Ik heb enorm respect voor hoe jij alles altijd weet te combineren. Dat jouw bizar drukke schema soms resulteerde in het feit dat ik mogelijke au-pair dossiers stond uit te printen of materialen voor Timmetjes spreekbeurt aan het verzamelen was, neem ik helemaal voor lief. Ik hoop de komende jaren nog veel meer van je te mogen leren! Heel erg bedankt voor alle begeleiding!

Geachte prof. Geelkerken, prof. Beets, prof. De Wilt, dr. Groot Jebbink, dr. Tanis en dr. Van Hooft, hartelijk dank dat jullie bereid zijn geweest om mijn proefschrift te beoordelen!

Beste Werner, laten we eerlijk zijn, in het begin moesten we even aan elkaar wennen. Gelukkig is dat uiteindelijk meer dan goed gekomen en hebben we elkaar helemaal gevonden! Ik wil je ontzettend bedanken voor je kritische blik. Je bent altijd ontzettend scherp en ziet snel waar onduidelijkheden zitten. Naast werk en onderzoek ben je daarnaast ook altijd scherp op de dansvloer. We blijven wel tot het einde he??

Beste Paul, jij straalt altijd enthousiasme en vrolijkheid uit en lijkt niet chagrijnig te krijgen. Jouw kritische maar vooral ook klinische blik op mijn manuscripten of onderzoeksprotocollen heeft me 
altijd enorm geholpen. Je bent enorm toegankelijk en ik hoop in de toekomst nog veel met je samen te mogen werken! Ik vind het echt top dat de PRINCE studie uiteindelijk van de grond is gekomen, mede dankzij jouw input!

Beste dr. ter Borg, beste Frank, mijn aller eerste colon stent procedure heb ik in Deventer mogen zien. Wat was het super om een keer in de kliniek te kunnen zien waar ik altijd over schrijf! Bedankt voor de kritische noot bij onze gezamenlijke artikelen.

Beste dr. Tanis, beste Pieter, ik denk dat er weinig mensen zijn die zó veel weten en zo up-to-date zijn van alle literatuur als jij. Heel erg bedankt voor de begeleiding bij al mijn projecten en natuurlijk met name het SNAPSHOT project. Ik ben er van overtuigd dat deze studie nooit zo van de grond was gekomen zonder jouw inzet! Ik denk dat we hiermee enorm waardevolle nieuwe data en informatie hebben gevonden. Heel veel dank!

Beste prof. Vriens, beste Menno, met open armen heb je me ontvangen in het UMCU waar ik het afgelopen jaar met enorm veel plezier als ANIOS heb gewerkt. Je enthousiasme en vrolijkheid werken motiverend en ik kan niet wachten om in januari in het UMC te mogen starten met de opleiding. Dank voor je vertrouwen!

Beste Jacob, je enthousiasme en bereidheid om altijd dingen uit te leggen bewonder ik enorm! Dank voor je hulp met de littekenbreuk echo's. Ik vind het mooi hoe we elkaar altijd overal weer tegenkomen, laten we dat erin houden.

Lieve Livia, mijn eerste wetenschaps-student, wat een primeur. En hoe goed heb je het gedaan, binnen een half jaar hebben wij er samen een super mooi artikel uitgeknald, heb je de Meander symposium prijs gewonnen en heb je gepresenteerd op de chirurgendagen. Je bent een topper en ik ben heel benieuwd naar je verdere carrière pad; heel veel succes in Boston!!

Beste Hector, heel erg bedankt voor je hulp en samenwerking bij mijn aller eerste artikel!

Paulus, we go way back. Als werkgroep 7 maten hebben wij onze hele master eigenlijk samen doorlopen. Hoe leuk dat je daarna ook nog in het Meander onderzoeksteam aansloot. Ik zal nooit vergeten dat je me op je afstudeerborrel na een $x$ aantal biertjes mee nam naar de puppies; veel gelukkiger kan je me niet maken. Tim, met jou is het altijd feest. Het maakt niet uit dat je misschien een afslagje te ver bent gegaan, over een bevroren meer moet lopen met ski's op je rug om vervolgens 20 liftjes te nemen en in 3 uur welgeteld 20 minuten te kunnen skiën; door jou heb ik alleen maar gelachen. En ff gezeten.. dat was ook wel fijn. Jan we hebben helaas nooit samen zitten bikkelen in de overdrachtsruimte, maar jouw droge humor maakt eigenlijk elke situatie! Robbie de technicus; ik ben blij dat je helemaal je plek hebt gevonden in Nijmegen; heel veel succes! Sander, collega's in de kliniek, tijdens onderzoek of samen naar een congres; we hebben het allemaal meegemaakt. Ik wens je heel veel succes met de laatste loodjes van jouw onderzoek en hoop snel jou aan te mogen moedigen in het mooie Twente.

En dan last, maar zeker niet least; Thijs, mijn opvolger. Ik had me geen betere opvolger kunnen wensen en vertrouw al mijn projecten aan jou toe. Je bent leergierig, neemt initiatief en bent altijd 
enthousiast. Ik vind het super om nog betrokken te blijven en ben stiekem ook wel heel blij dat je niet naar het AMC ontsnapt bent!

Lieve UMCU-MDL onderzoekers, als student kwam ik bij jullie op de kamer zitten en meteen voelde ik me welkom. Met veel plezier denk ik terug aan mijn tijd bij jullie. Lieve Jorrit, Joren, Faydra, Mirjam, Wouter en Yara; heel erg bedankt voor de mooie tijd!

Lieve Emo, mijn mede 'linkszijdige colon obstructie' onderzoeker! Tijdens mijn hele promotie onderzoek heb ik stiekem altijd ontzettend naar jou opgekeken. Je bent altijd vrolijk, super enthousiast, pakt dingen snel op en bent volgens mij super efficiënt. Ik vind het dan ook heel leuk dat wij uiteindelijk nog samen een mooi project hebben kunnen doen; de SNAPSHOT! Ik heb altijd met heel veel plezier met je samen gewerkt en wens je heel veel succes met je opleiding MDL. Ik weet zeker dat we elkaar in de toekomst nog zullen spreken!

Beste Wernard, samen met Emo hebben wij met zijn drieën de $2^{\mathrm{e}}$ nationale SNAPSHOT opgezet. Zonder jouw ervaring, begeleiding, 30+ emails met SNAPSHOT voorbeeld informatie en ondersteuning hierin was dat nooit gelukt!

Joyce! Jouw eerste kennismaking met de SNAPSHOT zal ik niet snel vergeten. Een ritje op en neer naar Zeeland om 'even' 60 patienten in te vullen. Door keihard door te knallen hebben we dat zowaar voor $18 \mathrm{u}$ gehaald. Wat een team. Ik vind het super dat ik via jou nauw betrokken blijf bij het SNAPSHOT project en weet zeker dat hier heel wat mooie publicaties uit voort gaan komen!

Beste Thijs Flinsenberg, jij bent de basislegger van mijn wetenschappelijke carrière. Als tweedejaars geneeskunde student kwam ik bij jou omdat ik graag onderzoek wilde doen naar stamcellen. Wat heb ik veel geleerd van die periode in het lab! Je zei eens tegen me; 'Om te promoveren moet je er echt voor willen gaan en continu bezig zijn met je onderzoek; in de douche krijg je vaak de beste ingevingen!'. Hoewel Fcy-receptoren natuurlijk reuze interessant zijn, had ik dat toch meer bij de chirurgie; ik snap nu helemaal waar je het over had! Thijs, heel erg bedankt voor je altijd ontzettend enthousiaste begeleiding! Oh en de wheelie op je motor, dat zal ik ook nooit vergeten.

Anja, Marja en Henriette; dank voor al jullie (administratieve en logistieke) hulp de afgelopen jaren!

Lieve Syl; dank dank dank voor al je wijze levenslessen en het feit dat je de EBP master dragelijk hebt gemaakt voor me! You are awesome.

Beste Pieternal Pasker, heel veel dank voor al je geduld en uitleg als het aankwam op statistiek! Ik heb met veel plezier met je samengewerkt, waarbij het helpen organiseren en presenteren van de Meander wetenschapsdag een duidelijk hoogtepunt was! Dank!

Lieve meander collega's, a.k.a. MOPPIES! Lieve Cassie, Roy, Henk, Oki, Franky, Roxy, Do, Sheila, Dien, Kluijf, Sabri, Snell, Slof, Katy Perry, Sven, Briet, Sander, Em, Arne en Clau. Wat zijn jullie een schatten! De vaatbespreking of de onco overnemen als ik weer eens de collegebanken in moest, het vragen van informed consent of het invullen van een CRF. Niks was jullie te gek; allemaal onwijs 
bedankt voor de inzet en het meedenken. Ik heb met volle teugen genoten van mijn tijd als ANIOS met jullie allemaal! Ik krijg een instant smile op mijn gezicht als ik terug denk aan de skireizen en de vele drink avonden die vrijwel altijd eindigde in totale escalatie!

Lieve UMCU collega's, dank voor de leerzame en bovenal gezellige tijd! De collegialiteit in deze groep is bewonderingswaardig wat de sfeer top maakt. Ik kan niet wachten om mijn eerste opleidingsjaar hier te mogen beginnen. Hoef ik jullie dus gelukkig nog niet te verlaten!

Lieve Frenkie! Jut en Jul op de D40; één team, één taak. Thanks voor de cover dotje!! Ik vind hem awesomeballs! :)

Pollie!! Onze burger en beer avondjes zijn iets wat we er voor altijd in moeten houden. Ik vind het zo leuk dat je, na wat omwegen, toch weer bij de geneeskunde terecht bent gekomen. Als je inderdaad anesthesist gaat worden hoop ik heel wat uren met je op OK door te mogen brengen. Wat zouden wij een dream team zijn.

Lieve lieve (oud) roomies van de Villa! Lieve Eddie, Fiep, Puk, Noud, Jip, Duuc en Mex. Bedankt voor het aanhoren van de vele powerpoint presentaties! Hoewel jullie er waarschijnlijk weinig tot niks van begrepen, hebben jullie me altijd gesteund. Mooiste huis van het Sticht! Louter liefde voor jullie.

Lieve Jippie en Noud, my special granny friends, aan jullie - natuurlijk - nog een eigen stukje. Er kunnen 8 Jeroens, 10 Brams of een sloot aan bouwvakkers en loodgieters langskomen; het mental trio is unbreakable. Onvergetelijk en priceless; zo zou ik onze avonturen wel willen beschrijven. Van wintersport naar love island marathons, alles klopt (als er maar KIIIIP is). Onvoorwaardelijk schatjes; voor altijd!

Claire, Juud, Kir, Minou, Lot en Maaik; inmiddels zijn we bij de Croese 4.0 app aanbeland (GOZERWIJFFIEEEES) maar jullie hebben allemaal moeten dealen met mijn onderzoek perikelen, die er soms (lees: regelmatig) toe hebben geleid dat er een (lees: elke) huisbeurt erbij in is geschoten; oprechte excuses dotjes. Duizend maal dank voor alle chill (en drink) avondjes die me door dit traject hebben gesleept!

Char! Chillpak aan en gaan. En wat ben je fotogeniek.. Ik kan niet wachten tot we straks (in bilthoven?) naast elkaar wonen, want na onze croese bonding time kan ik niet meer zonder jou in je roze onesie.

Mariee, MC, Mary, Eck, Claire (als je vervelend bent); wij voelen elkaar aan. Aan 1 blik hebben wij meer dan genoeg. Of mijn aura nou zwart of roze is jij mag me altijd knuffelen. Ik vind jou lief.

Lieve Bruisjes, wat een toppers zijn jullie. Nachtdiensten, weekenddiensten of onderzoeksdienst staat vaak ik de weg van onze dates. Maar dat maakt niks uit voor wanneer we dan weer bij elkaar zijn. Ik vind het altijd heerlijk bij jullie en kan helemaal mezelf zijn; misschien zelfs een beetje te veel. Schatten: dank voor alle mooie herinneringen so far, ik kan niet wachten op de rest! Ps. Ik wil nog steeds een dwerghamster en dan noem ik hem Bruce. 
Lieve Hannie en Innie, hoeveel geluk heb ik met twee tantes. Heerlijk vind ik het om soms gezellig even langs te komen in Den Dolder voor wat rust en een heerlijke (vegan) maaltijd. Ik hoop dat dat in de toekomst nog veel meer wordt, nu deze drukke periode eindelijk klaar is! Dank voor jullie onvoorwaardelijk support.

Lieve omi, dank voor je altijd luisterende oor. Ik weet altijd dat er in Wijchen een kopje thee en gebakje op me wacht waar ik even aan de drukte kan ontsnappen. Dank lieve omi; hopelijk kan ik nu nog vaker langs komen.

Allerliefste paranimfen Hendrike en Denise, lieve Dirkie en Den; hoe fijn om jullie vandaag aan mijn zijde te hebben. Na de fantastische promoties van jullie twee mag ik de cirkel rond maken. We voelen elkaar aan, juist doordat we alle 3 in hetzelfde schuitje hebben gezeten. Promoveren naast werk is soms lastig uit te leggen, maar bij jullie is 1 woord voldoende.

Lieve Hendrike, Dirkie, we kennen elkaar al zo lang!! Hoewel we elkaar even uit het oog zijn verloren, hebben we elkaar weer helemaal gevonden. Hoe mooi waren al onze congres tripjes!! Jij als een vis in het water en ik hinkend in de kittykat club natuurlijk als hoogtepunt. Zelfs het missen van een vliegtuig kan bij ons de pret niet drukken. Boeken we toch een vluchtje later vanuit Roffa? No stress. Dirkie; je hebt me altijd feilloos door, nog voordat ik dat zelf weet. Je advies is altijd on point en door jou neem ik tegenwoordig het heft in eigen handen. Dirkie je bent een topper!

Lieve Denise, Dennie, Den, de mamma van de groep. Als de dag van gister weet ik dat je tegen me zei dat ik je altijd alles kon vragen; wat een chill gevoel. Je hebt me door die begin ANIOS periode heen gesleept en staat altijd voor me klaar. Geen sprekender voorbeeld is de Dam tot Dam; zonder jouw support had ik die laatste $3 \mathrm{~km}$ tot aan de finish never ever gehaald. Je weet precies hoe je iemand moet motiveren en bent een bom aan vrolijke en aanstekelijke energie. Ik vind het zo knap dat je er helemaal uit bent wat je wil en steun je $100 \%$ !!

Lieve lieve Xan, hoewel je alleen het staartje hebt meegekregen van dit bizarre 6-jarige project genaamd: "promoveren in je vrije tijd", heb ik me nooit meer gesteund gevoeld dan in die laatste maanden. Je bent oprecht geïnteresseerd en weet precies waar ik me mee bezig houd. Hoewel er heel wat avondjes en weekenddagen opgeofferd moesten worden, ben je altijd begripvol. Sterker nog, je steunt en stimuleert me altijd om alles eruit te halen. Schat, samen met jou heb ik het gevoel dat ik de wereld aan kan. Ik kan niet wachten op alles wat nog komen gaat; dit wordt onze tijd.

Lieve Linnie, Lieve Jus, mijn kleine zusjes! Wat zijn jullie een kanjers. Onze sista chill avonden geven me altijd energie en een boost om een drukke periode door te komen. Ik weet dat ik altijd bij jullie terecht kan, ook om gewoon even te klagen. Ik ben zo blij met hoe hecht onze band is en kijk uit naar al onze avonturen. Sista love!

Lieve Mamma, Lieve Pappa. Mijn dankbaarheid naar jullie kan ik niet in woorden uitdrukken. Altijd hebben jullie me gestimuleerd om het beste uit mezelf te halen en daar pluk ik de rest van mijn leven de vruchten van. Van jullie heb ik geleerd dat je met voldoende inzet en discipline vrijwel alles kunt bereiken wat je wil. Door jullie onvoorwaardelijke liefde, steun en support heb ik hier ook altijd in geloofd. Zonder jullie was dit proefschrift er niet geweest. 


\section{PUBLICATION LIST}

F.J. Amelung, C.L.J. Mulder, P.M. Verheijen, W.A. Draaisma P.D. Siersema, E.C.J. Consten. Acute resection versus bridge to surgery with diverting colostomy for patients with acute malignant left sided colonic obstruction: systematic review and meta-analysis. Surgical Oncology 2015;24(4):313-21

F.J. Amelung, H. De Beaufort, P.D. Siersema, P.M. Verheijen, E.C.J. Consten . Emergency resection versus bridge to surgery with stenting in patients with acute right-sided colonic obstruction: a systematic review focusing on mortality and morbidity rates. International Journal of Colorectal Disease 2015;30(9):1147-55

F.J. Amelung, E.C.J. Consten, P.D. Siersema, P.J. Tanis. A population based analysis of three treatment modalities for malignant obstruction of the proximal colon: acute resection versus stent or stoma as a bridge to surgery. Annals of Surgical Oncology 2016;23(11):3660-3668

F.J. Amelung, F. ter Borg, E.C.J. Consten, P.D. Siersema, W.A. Draaisma. Deviating colostomy vs stent placement as bridge to surgery for malignant left-sided colonic obstruction. Surgical Endoscopy 2016;30(12):5345-5355

F.J. Amelung, C.L.J Mulder, E.C.J. Consten, W.A. Draaisma. Efficacy of loop colostomy construction for acute left-sided colonic obstructions: a cohort analysis. International Journal of Colorectal Disease 2017;32(3):383-390

F.J. Amelung, C.P. van t Hullenaar, P.M. Verheijen, E.C.J. Consten. Ileostomy versus colostomy: which is preferable? Nederlands Tijdschrift voor Geneeskunde, 2017;161(0):D788

F.J. Amelung, W.A. Draaisma, E.C.J. Consten, P.D. Siersema, F. ter Borg. Self-expandable metal stent placement versus emergency resection for malignant proximal colon obstructions. Surgical Endoscopy 2017;31(11):4532-4541

F.J. Amelung, L.E.V.M. de Guerre, E.C.J. Consten, P.M. Verheijen, I.A.M.J. Broeders, W.A. Draaisma. Incidence and risk factors of ostomy site incisional hernia following stoma reversal. British Journal of Surgery Open 2018;2:128-134

F.J. Amelung, T.A. Burghgraef, P.J. Tanis, J.E. van Hooft, F. ter Borg, P.D. Siersema, W.A. Bemelman, E.C.J. Consten. Long-term outcomes following stent placement as bridge to surgery compared to emergency surgery in patients with acute left-sided colonic obstruction; an updated meta-analysis. Critical Review in Hematology Oncology 2018

F.J. Amelung, W.A.A. Borstlap, E.C.J. Consten, J.V. Veld, E.E. van Halsema, J. van Hooft, W.A. Bemelman, P.D. Siersema, F. ter Borg, P.J. Tanis Long-term outcomes following SEMS placement as bridge to surgery versus a surgical approach for left-sided colonic obstructions. Submitted 


\section{PUBLICATIONS OUTSIDE OF THIS THESIS}

Flinsenberg TQ, Compeer EB, Koning D, Klein M, Amelung FJ, van Baarle D, Boelens JJ, Boes $M$. Fcy receptor antigen targeting potentiates cross-presentation by human blood and lymphoid tissue BDCA-3+ dendritic cells. Blood 2012;20;120(26):5163-72

F.J. Amelung, G.A. Zoet, T.E. Vogelvang. Varicocèle behandeling en de invloed op de androgene fertiliteit. NTVOG 2013; 126: 461-465

Jonker FHW, Hagemans JAW, Burger JWA, Verhoef C, Borstlap WAA, Tanis PJ; Dutch Snapshot Research Group. The influence of hospital volume on long-term oncological outcome after rectal cancer surgery. Int J Colorectal Dis. 2017 Dec;32(12):1741-1747

Borstlap WAA, Westerduin E, Aukema TS, Bemelman WA, Tanis PJ; Dutch Snapshot Research Group. Anastomotic Leakage and Chronic Presacral Sinus Formation After Low Anterior Resection: Results From a Large Cross-sectional Study. Ann Surg. 2017 Nov;266(5):870-877

Dutch Snapshot Research Group. Benchmarking recent national practice in rectal cancer treatment with landmark randomized controlled trials. Colorectal Dis. 2017 Jun;19(6):0219-0231

EuroSurg Collaborative. Body mass index and complications following major gastrointestinal surgery: A prospective, international cohort study and meta-analysis. Colorectal Dis. 2018 Jun 13.

Blok RD, Musters GD, Borstlap WAA, Buskens CJ, Bemelman WA, Tanis PJ; Collaborative Dutch Snapshot Research Group. Snapshot Study on the Value of Omentoplasty in Abdominoperineal Resection with Primary Perineal Closure for Rectal Cancer. Ann Surg Oncol. 2018 Mar;25(3):729736 


\section{CURRICULUM VITAE}

Femke Julie Amelung was born on June 18th 1990 in Utrecht, the Netherlands as the first of three sisters. She grew up in Utrecht, Brasschaat and Zeist, and graduated with honours in 2008 from high school (Utrechts Stedelijk Gymnasium). After a year of travelling Spain, Australia and the UK she started medical school in September 2009 at the University of Utrecht. During her studies she was always interested in research and simultaneous to her bachelor courses she participated in a research project at the Immunology department. Her interest in Surgery emerged during her junior internship at the Surgical department at the Antonius Hospital. Herafter, she concluded her research in Immunology and continued further research projects in the field of Surgery.

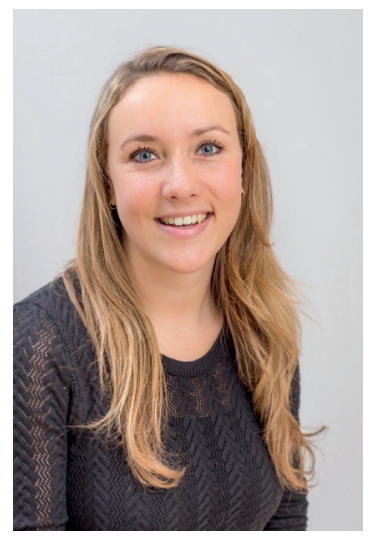

Throughout her final years of medical school, she conducted the first studies of what is now the base of this thesis under the supervision of dr. Consten, prof. Broeders and prof. Siersema. In February 2016 she obtained her medical degree, whereafter she continued with fulltime research for five months. In August 2016 she began working as a surgical resident (not in training) at Meander Medical Center. During her clinical work, she continued with her thesis. In order to increase her statistical and methodological knowledge, she started an additional master in Epidemiology, which she completed with honours in June 2018. From January 2018 she worked as a surgical resident (not in training) at the University Medical Center Utrecht. In January 2019 she will start as a surgical resident in training. She will remain actively engaged as a supervisor of the two $\mathrm{PhD}$ candidates that continue her research projects. 



If
You
Haven't
Found
It
Yet
Keep
Looking

